\title{
Modelos de regressão para dados censurados sob Distribuições Simétricas
}

\author{
Aldo William Medina Garay \\ TESE APRESENTADA \\ $\mathrm{AO}$ \\ Instituto de Matemática e Estatística \\ DA \\ Universidade DE SÃo PaUlo \\ PARA \\ OBTENÇÃO DO TÍTULO \\ $\mathrm{DE}$ \\ Doutor EM Ciencias \\ Programa: Estatística \\ Orientador: Prof. Dr. Heleno Bolfarine \\ Co-orientador: Prof. Dr. Víctor Hugo Lachos Dávila
}

Este trabalho foi financiado pela CAPES e CNPq

São Paulo, Abril de 2014 


\section{Modelos de regressão para dados censurados sob Distribuições Simétricas}

Este exemplar corresponde à redação

final da tese devidamente corrigida e defendida por Aldo William Medina Garay e aprovada pela Comissão Julgadora.

Banca Examinadora:

- Prof. Dr. Heleno Bolfarine.

- Prof. Dr. Víctor Hugo Lachos Dávila.

- Prof. Dr. Marcos Oliveira Prates.

- Prof. Dr. Filidor Edilfonso Vilca Labra.

- Prof. Dr. Edwin Moisés Marcos Ortega. 


\section{Dedicatória}

À minha mãe Alejita e meu tio Fabián, com admiração; à meu pai Carlos (in memoriam), com saudade; à minha esposa Fran Lima, com amor e complicidade; à meus irmãos Carlos, Nora e Lili, com carinho. 


\section{Agradecimentos}

- Em primeiro lugar agradeço a minha mãe por seu sacrifício, entrega, esforço e dedicação para me educar, guiar e ser meu melhor exemplo de vida.

- Ao meu orientador o Professor Heleno Bolfarine, um excelente pesquisador e pessoa, que sempre me motivou a seguir pelo caminho da pesquisa e do trabalho acadêmico.

- À meu coorientador e amigo o Professor Victor Hugo Lachos Dávila, pela sua dedicação, exemplo e em especial sua amizade nestes últimos 7 anos.

- À minha esposa Fran Lima Medina pelo seu amor, carinho, compreensão, paciência e por estar sempre a meu lado.

- A todos os professores da banca pelas suas sugestões e contribuições importantes para que este trabalho pudesse ser melhorado.

- Ao professor Celso Rômulo Barbosa Cabral, pela ajuda e conselho, sua experiência foi muito importante na obtenção dos resultados finais da pesquisa. Agradeço sua disposição para me ajudar nos momentos difíceis da elaboração desta tese.

- Aos professores do Departamento de Estatística do IME-USP, pelos ensinamentos concedidos.

- À Jorge Torrejón, Danilo Figuereido, Diego Tonin, Rodrigo Lambert e Monique Massuia pela sua amizade, apoio e disposição para me ajudar e acompanhar sempre.

- À minha família peruana: Alejita, Fabián, Nora, Lili, Carlos e todos meus sobrinhos que sempre me apoiaram e estiveram comigo no coração e na minha mente.

- À minha família brasileira de Caarapó: Ionice, Aparecido, Carla, Fredinho, Zé, Tata e madrinha Zê por me acompanhar nestes anos e ser parte da minha história de vida.

- À CAPES e CNPq pelo apoio financeiro.

- Acima de tudo agradeço a Deus, pela oportunidade de estar aqui e pela dádiva da vida. 


\section{Resumo}

Este trabalho tem como objetivo principal apresentar uma abordagem clássica e Bayesiana dos modelos lineares com observações censuradas, que é uma nova área de pesquisa com grandes possibilidades de aplicações. Aqui, substituimos o uso convencional da distribuição normal para os erros por uma família de distribuições mais flexíveis, o que nos permite lidar de forma mais adequada com observações censuradas na presença de outliers. Esta família é obtida através de um mecanismo de fácil construção e possui como casos especiais as distribuições t de Student, Pearson tipo VII, slash, normal contaminada e, obviamente, a normal.

Para o caso de respostas correlacionadas e censuradas propomos um modelo de regressão linear robusto baseado na distribuição $t$ de Student, desenvolvendo um algoritmo tipo EM que depende dos dois primeiros momentos da distribuição $t$ de Student truncada.

Palavras-chave: Modelos de Regressão censurados, Algoritmo tipo EM, Modelagem Bayesiana, Algoritmo tipo MCMC, Misturas da escala da distribuição normal, estrutura de correlação. 


\section{Abstract}

This work aims to present a classical and Bayesian approach to linear models with censored observations, which is a new area of research with great potential for applications. Here, we replace the conventional use of the normal distribution for the errors of a more flexible family of distributions, which deal in more appropriately with censored observations in the presence of outliers. This family is obtained through a mechanism easy to construct and has as special cases the distributions Student t, Pearson type VII, slash, contaminated normal, and obviously normal.

For the case of correlated and censored responses we propose a model of robust linear regression based on Student's t distribution and we developed an EM type algorithm based on the first two moments of the truncated Student's t distribution.

Keywords: Censored regression models, EM-type algorithm, Bayesian modeling, MCMCtype algorithm, Scale mixtures of normal distribution, correlation structure. 


\section{Sumário}

1 Introdução 1

$1.1 \quad$ Preliminares . . . . . . . . . . . . . . . . . . . . . . . 1

1.1 .1 Truncamento . . . . . . . . . . . . . . . . . . 1

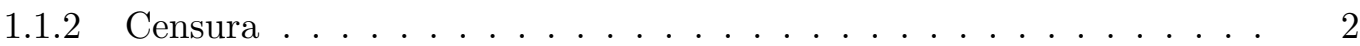

1.2 Distribuições misturas da escala da normal . . . . . . . . . . . . . . 2

1.2 .1 Definições . . . . . . . . . . . . . . . . . . . 2

1.2.2 Exemplo de distribuições SMN . . . . . . . . . . . . . . . . 4

1.3 Algoritmo EM . . . . . . . . . . . . . . . . . . . 8

1.4 Estimação Bayesiana . . . . . . . . . . . . . . . . . . . . . . . . . 9 9

1.4.1 Amostrador de Gibbs . . . . . . . . . . . . . . . . . . . . 10

1.4.2 O algoritmo Metropolis-Hastings . . . . . . . . . . . . . . 11

2 Modelos SMN-CR 13

2.1 Introdução . . . . . . . . . . . . . . . . . . . . . . . . . . . . 13

2.2 Descrição do modelo . . . . . . . . . . . . . . . . . . . . . . . 14

2.3 Função de $\log$-verossimilhança . . . . . . . . . . . . . . . . . . . . . . . 15

2.4 Algoritmo EM nos modelos SMN-CR . . . . . . . . . . . . . . . . . 15

2.5 Erro padrão aproximado para os efeitos fixos . . . . . . . . . . . . . 18

2.6 Seleção de modelos . . . . . . . . . . . . . . . . . . . . . . . . . . . 19

2.7 Análise de resíduos . . . . . . . . . . . . . . . . . . . . . . . . . . . 19

2.8 Estudos de Simulação . . . . . . . . . . . . . . . . . . . . . . . . 20

2.8.1 Simulação 1: Robustez das estimativas de MV . . . . . . . . . . 20

2.8.2 Simulação 2: Propriedades Assintóticas . . . . . . . . . . . . . . . . 21

2.8.3 Simulação 3: Performance dos Erros Padrões . . . . . . . . . . . . . 22

2.9 Pacote SMnCensReg . . . . . . . . . . . . . . . . . . . . . . 24

2.9 .1 Introdução . . . . . . . . . . . . . . . . . . . . . . . . 24

2.9.2 Descrição . . . . . . . . . . . . . . . . . . . . 24

2.9 .3 Utilização do pacote . . . . . . . . . . . . . . . . . 25

2.10 Aplicação: Salários . . . . . . . . . . . . . . . . . . . . . . . . . . . 27

2.10 .1 Ajuste do modelo . . . . . . . . . . . . . . . . . . . 28

2.10 .2 Análise de resíduos . . . . . . . . . . . . . . . . . 28

2.10 .3 Robustez das estimativas de MV . . . . . . . . . . . . . . . 29 


\section{Estimação Bayesiana dos modelos SMN-CR}

3.1 Introdução . . . . . . . . . . . . . . . . . . . . . . . . . . . . 31

3.2 Inferência Bayesiana nos modelos SMN-CR . . . . . . . . . . . . . 32

3.2 .1 Distribuições a priori . . . . . . . . . . . . . . . . . 32

3.2 .2 Estimação via MCMC . . . . . . . . . . . . . . . . . . . . . . 32

3.3 Critérios de comparação de modelos . . . . . . . . . . . . . . 36

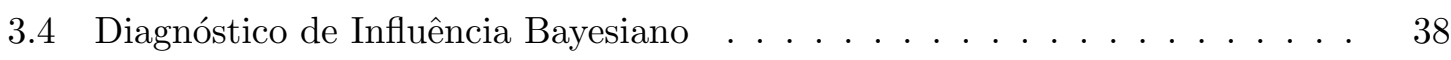

3.5 Estudos de simulação . . . . . . . . . . . . . . . . . . . . . . . . . 39

3.5.1 Simulação $1 \ldots \ldots \ldots . \ldots \ldots \ldots$

3.5.2 Simulação $2 \ldots \ldots \ldots$. . . . . . . . . . . . . . . 41

$3.6 \quad$ Pacote BayesCR . . . . . . . . . . . . . . . . . . . . . . . . . 43

3.6 .1 Introdução . . . . . . . . . . . . . . . . . . . . 43

3.6 .2 Descrição . . . . . . . . . . . . . . . . . . . . . 44

3.6.3 Utilização do pacote . . . . . . . . . . . . . . . . . . . . 44

3.7 Aplicação: Salários . . . . . . . . . . . . . . . . . . . . . . 46

3.7 .1 Estimação . . . . . . . . . . . . . . . . . . . . . . 46

3.7.2 Diagnóstico de influência Bayesiana . . . . . . . . . . . . . . 48

4 Modelos SMN-CNLR

4.1 Introdução . . . . . . . . . . . . . . . . . . . . . . . . 51

4.2 Modelo de regressão não linear censurado SMN . . . . . . . . . . . . . . 52

4.2 .1 Descrição do modelo . . . . . . . . . . . . . . . . 52

4.2 .2 Função de log-verosimilhança . . . . . . . . . . . . . . . . . . 52

4.3 Estimação dos parâmetros . . . . . . . . . . . . . . . . . . 53

4.3.1 O algoritmo ECME para os modelos SMN-NLCR . . . . . . . . . 53

4.3 .2 Especificação dos valores iniciais . . . . . . . . . . . . . 54

4.4 Erro padrão aproximado para os efeitos fixos . . . . . . . . . . . . 55

4.5 Estudos de simulação . . . . . . . . . . . . . . . . 55

4.5.1 Simulação 1: Propriedades Assintóticas . . . . . . . . . . . . . . 56

4.5.2 Simulação 2: Performance dos Erros Padrões . . . . . . . . . . . . . 56

4.5.3 Simulação 3: Desempenho das estimativas de MV . . . . . . . . . 57

4.6 Aplicação: Calibração ultrassônica . . . . . . . . . . . . . . . . . . . . . . 59

5 Modelos T-MLC

5.1 Introdução . . . . . . . . . . . . . . . . . . . . . . . 63

5.2 Motivação: Dados UTI . . . . . . . . . . . . . . . . . . . . . . . 65

5.3 Especificação do modelo . . . . . . . . . . . . . . . . . 66

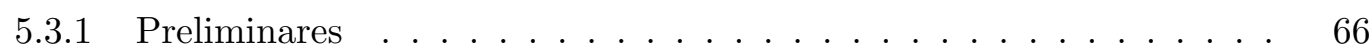

5.3 .2 Descrição do modelo . . . . . . . . . . . . . . . . . . . 68

5.3 .3 Função de log-verosimilhança . . . . . . . . . . . . . . . . . 70

$5.4 \mathrm{O}$ algoritmo EM . . . . . . . . . . . . . . . . . 70 
5.4.1 Imputação dos casos censurados via o algoritmo ECM . . . . . . . . 73

5.5 Erro padrão aproximado para os efeitos fixos . . . . . . . . . . . . 73

5.6 Predição de valores futuros . . . . . . . . . . . . . . . . . 75

5.7 Estudos de simulação . . . . . . . . . . . . . . . . . 76

5.7.1 Simulação 1: Imputação das observações censuradas . . . . . . . 76

5.7.2 Simulação 2: Propriedades Assintóticas . . . . . . . . . . . . 77

5.8 Aplicação . . . . . . . . . . . . . . . . . . . . . . . 78

6 Conclusões Finais e Perspectivas Futuras $\quad 83$

6.1 Conclusões Finais . . . . . . . . . . . . . . . . . . . . . . . . 83

6.2 Perspectivas Futuras . . . . . . . . . . . . . . . . . . . 84

A Lemas e Corolários $\quad 85$

$\begin{array}{lll}\text { B Cálculo de } \boldsymbol{E}_{\phi}(r, h) \text { e } \boldsymbol{E}_{\Phi}(r, h) & \mathbf{8 7}\end{array}$

C Detalhes do algoritmo EM $\quad 89$

$\begin{array}{ll}\text { D Propriedades assintóticas } & 91\end{array}$

$\begin{array}{lll}\text { E Algoritmo EM para o modelo t-MLC } & 93\end{array}$

$\begin{array}{lll}\text { F Prova das Proposições } 3 \text { e } 4 & 95\end{array}$

$\begin{array}{ll}\text { Referências Bibliográficas } & 96\end{array}$ 


\title{
Lista de Abreviaturas
}

\author{
SMN Misturas da escala da normal. \\ MV Máxima verossimilhança. \\ CR Regressão censurada. \\ fdp Função Densidade de Probabilidade. \\ fda Função de Distribuição Acumulada. \\ g.l. Graus de liberdade. \\ TN Normal truncada. \\ MCMC Cadeias de Markov via Monte Carlo. \\ EP Erro padrão. \\ DEC Correlação damped exponential. \\ MLC Modelo Linear Censurado Multivariado. \\ MAE Erro Médio Absoluto. \\ EQM Erro Quadrático Médio.
}




\section{Lista de Figuras}

1.1 Densidade da classe de distribuições SMN . . . . . . . . . . . . . . . 5

2.1 Mudança relativa média nas estimativas de MV para diferentes valores de $\vartheta . \quad 21$

2.2 Viés médio (primeira linha) e EQM (segunda linha) dos parâmetros estimados

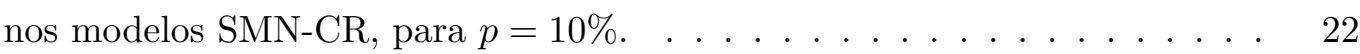

2.3 Envelopes dos resíduos martingais transformados $r_{M T_{i}}$, para os modelos

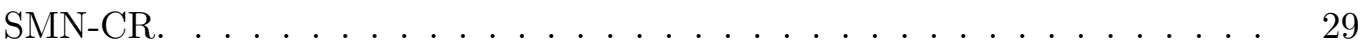

2.4 Mudanças relativas nas estimativas de MV dos modelos SMN-CR para diferentes contaminações $\nabla$ das observações $y_{7}\left(1^{a}\right.$ linha $)$ e $y_{750}\left(2^{a}\right.$ linha $) \ldots$.

3.1 MC Média dos parâmetros $\beta_{1}, \beta_{2}$ e $\sigma^{2}$ para os modelos N-CR e T-CR em comparação com o verdadeiro valor dos parâmetros (Linha vermelha) . . . .

3.2 Gráfico de caixas do MC Média dos parâmetros $\beta_{1}$ e $\beta_{2}$, sob os modelos (a) N-CR e (b) T-CR. A linha vermelha representa o verdadeiro valor dos parâmetros. . . . . . . . . . . . . . . . . . .

3.3 Estudo de simulação: (a) RELMB e (b) RRELMSE sob diferentes a priori

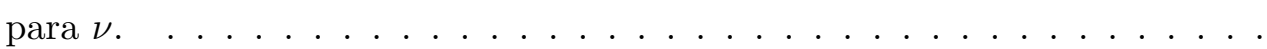

3.4 Histórico das cadeias e densidades marginais a posteriori aproximadas, considerando o modelo SL-CR . . . . . . . . . . . . . . . . . . . .

3.5 Medidas de diagnóstico de influência para os modelos N-CR (1ra Linha), T-CR (2da Linha) e SL-CR (3ra Linha): (a) K-L divergência, (b) distância J, (c) distância $L_{1}$

4.1 Viés e EQM médio para os parâmetros estimados . . . . . . . . . . . . . 57

4.2 Gráfico de dispersão para os dados de calibração ultrassônica com censura $\quad 60$

5.1 Dados UTI. (a) Perfil de cada indivíduo (na escala $\log _{10}$ ) segundo a carga viral. A trajetória de três indivíduos censurados são marcadas. (b) $Q Q$ plot para os resíduos do modelo normal. . . . . . . . . . . . . . .

5.2 Simulação 1. Média do (a) MAE e (b) EQM sobre $M=100$ conjuntos de dados do modelo $t$-MLC com estrutura de correlação U e UNC. . . . . . . 77

5.3 Simulação 2. Viés ( $1^{\text {ra }}$ linha) e EQM ( $2^{\text {da }}$ linha) dos parâmetros estimados no modelo $t$-MLC com $5 \%$ de censura. . . . . . . . . . . 78

5.4 Dados UTI. Log-verossimilhança perfilada dos graus de liberdade $\nu$. . . . 79 
5.5 Dados UTI. Pesos estimados $\widehat{u}_{i}$ para o modelo $t$-MLC.

5.6 Dados UTI. Evaluação da performance da predição para três indivíduos escolhidos aleatóriamente. . . . . . . . . . . . . . . . . . . . 82

D.1 Viés médio (primeira linha) e EQM (segunda linha) dos parâmetros estimados nos modelos SMN-CR, para $p=25 \%$. . . . . . . . . . .

D.2 Viés médio (primeira linha) e EQM (segunda linha) dos parâmetros estimados nos modelos SMN-CR, para $p=45 \% \ldots \ldots \ldots \ldots$ 


\section{Lista de Tabelas}

2.1 Erro padrão amostral de $\widehat{\beta}_{i}$ (MC EP), MC IM EP e COV MC. . . . . . . . 23

2.2 Estimativas de MV e erros padrões (EP) obtidos no ajuste de vários modelos SMN-CR para os Salários. . . . . . . . . . . . . . . . . . 28

2.3 Critérios de seleção de modelos. . . . . . . . . . . . . . . . . . . . . 28

3.1 MC média, MC DP (Em parêntese) e MC Cov para os modelos T-CR e N-CR com diferentes níveis de censura. . . . . . . . . . . . . . . . 40

3.2 Viés médio relativo (RELMB) e error quadrático médio relativo (RRELMSE) para diferentes valores de $\nu$ considerando diferentes a priori. . . . . . . . . .

3.3 Média a posteriori, desvio padrão (DP) e intervalo HPD de $95 \%$ para os parâmetros dos modelos SMN-CR. . . . . . . . . . . . . . . . 47

3.4 Critérios de seleção de modelos. . . . . . . . . . . . . . . . . . . . 47

4.1 MC EP, MC IM EP e COV MC de $\widehat{\beta}_{i} \ldots \ldots \ldots \ldots \ldots$

4.2 Média e o Desvio Padrão (em parentese) do MMER. . . . . . . . . . . . . . 59

4.3 Estimativa de MV, Erro padrão estimado (EP) e um intervalo de confiança assintótico (IC) para os modelos SMN-NLCR. . . . . . . . . . . . . . . . 61

4.4 Comparação entre os modelos SMN-NLCR. . . . . . . . . . . . . . . . . . 61

5.1 Correlação obervada do $\log _{10} \mathrm{HIV}-1$ em diferentes tempos de avaliação. . . . 66

5.2 Simulação 1. Média do MAE e do EQM sobre os $M=100$ conjunto de dados. UNC: Sem estrutura de correlação e U: Parâmetros $\phi_{1}$ e $\phi_{2}$ da matriz

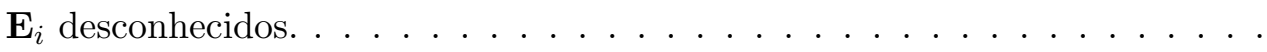

5.3 Dados UTI. Estimativas de MV e erro padrão (EP) para os coeficientes de regressão sob os modelos MLC normal e $t$ com estrutura de correlação DEC. UNC: Sem estrutura de correlação e U: Parâmetros $\phi_{1}$ e $\phi_{2}$ da matriz

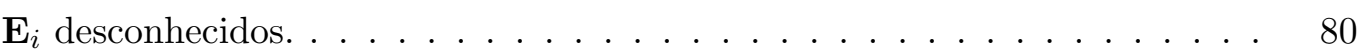

5.4 Dados UTI. Comparação entre os modelos MLC normal e $t$ de Student. UNC: Sem estrutura de correlação e U: Parâmetros $\phi_{1}$ e $\phi_{2}$ da matriz $\mathbf{E}_{i}$ desconhecidos. 
5.5 Dados UTI. Avaliação da precisão das predições considerando o modelo $t$ MLC com diferentes estruturas de correlação DEC. U: Parâmetros $\phi_{1}$ e $\phi_{2}$ da estrutura de corelação desconhecidos. . . . . . . . . . . . . . . . . 


\section{Capítulo 1}

\section{Introdução}

\section{$1.1 \quad$ Preliminares}

Ao longo deste trabalho utilizaremos algumas notações, as quais serão inicialmente denotadas neste capítulo.

Sendo assim, seja $X \sim \mathrm{N}\left(\mu, \sigma^{2}\right)$ uma variável aleatória seguindo distribuição normal com média $\mu$ e variância $\sigma^{2}$, e $\phi\left(\cdot \mid \mu, \sigma^{2}\right)$ denota a sua função densidade de probabilidade (fdp). $\phi(\cdot)$ e $\Phi(\cdot)$ denotam, respectivamente a fdp e a função de distribuição acumulada (fda) da normal padrão, isto é, no caso em que $\mu=0$ e $\sigma^{2}=1$.

Em geral, utilizamos a convenção tradicional denotando uma variável aleatória (ou um vetor aleatório) por uma letra maiúscula e sua realização pela letra minúscula correspondente. Vetores aleatórios e matrizes são representados por letras em negrito. $\mathbf{X}^{\top}$ é a transposição de $\mathbf{X}$. Assim também, $X \perp Y$ indica que as variáveis aleatórias $X$ e $Y$ são independentes.

Um princípio que adotaremos é denotar algumas distribuições por abreviaturas em inglês. Por exemplo, a distribuição normal truncada será referenciada por TN (truncated normal), a regressão censurada por CR (censored regression), e assim por diante.

Neste trabalho estudaremos os modelos de regressão quando a variável resposta apresenta observações incompletas, devido a censura. No entanto, é importante esclarecer a diferença entre as duas principais causas de dados incompletos: a censura e o truncamento, os quais serão utilizados constantemente ao longo do nosso texto.

\subsubsection{Truncamento}

O truncamento ocorre quando algumas observações, tanto na variável resposta como nas variáveis regressoras não estão disponíveis, isto é, o truncamento é caracterizado por uma condição que exclui certos indivíduos do estudo.

Por exemplo considerando o caso apresentado em Colosimo \& Giolo (2006), o truncamento ocorre se, para a estimação da distribuição do tempo de vida dos moradores de uma certa localidade, for usada uma amostra retirada do banco de dados da previdência local. Desta forma, somente moradores que atingiram a aposentadoria fazem parte da amostra. Assim, 
estas observações são conhecidas por truncadas à esquerda. Outros exemplos de truncamento podem ser encontrados em Nelson (1990), Kalbfleisch \& Lawless (1992).

As distribuições contínuas truncadas também são comumente usadas em outras áreas de pesquisa como economia (veja por exemplo Arnold \& Press (1989) e Dueker (2006)), hidrologia (Jawitz, 2004), entre outros.

\subsubsection{Censura}

A censura ocorre quando dados sobre a variável resposta não estão completamente disponíveis para algumas unidades da amostra, no entanto, para estas unidades, os dados sobre as variáveis regressoras são totalmente conhecidos. Em várias situações práticas, a censura acontece por razões tais como as limitações dos equipamentos de medição ou do projeto experimental. Assim, informalmente podemos dizer que uma observação censurada contém apenas informações parciais sobre um evento de interesse. Por exemplo a agulha de uma escala que não fornece uma leitura acima de $200 \mathrm{~kg}$ vai mostrar $200 \mathrm{~kg}$ para todos os objetos que pesam mais do que o limite. Outro exemplo interessante é extraído de Breen (1996): em um exame escolar, a porcentagem mínima de acertos para a aprovação é de 40\%. O certificado, contendo a situação do aluno (aprovado ou não) é dado a todos eles, mas apenas os alunos que aprovaram tem especificada a sua pontuação exata. Suponha que queremos estudar a relação entre as pontuações e algumas outras variáveis explicativas, como classe social, gênero e escolaridade dos pais. Neste caso, as pontuações são as respostas com censura à esquerda, porque, se $y_{i}$ denota a pontuação do $i$-ésimo aluno e ele ou ela não for aprovado(a), não conheceremos o valor exato de $y_{i}$, somente saberemos que este valor é menor do que $40 \%$.

Modelos envolvendo respostas censuradas com erros de observação normalmente distribuídos têm sido estudados extensivamente na literatura (veja por exemplo Nelson (1977), Stapleton \& Young (1984), Chib (1992), Thompson \& Nelson (2003), Park et al. (2007) e Vaida \& Liu (2009)). Em modelos de regressão linear censurados com distribuição t de Student, os trabalhos de Arellano-Valle et al. (2012) e Matos et al. (2013) podem ser mencionados, a partir de uma abordagem clássica.

No entanto, pouca atenção tem sido dada à questão dos erros de observação com uma classe de distribuição com caudas mais pesadas do que a normal, seja a partir de uma abordagem clássica ou Bayesiana.

\subsection{Distribuições misturas da escala da normal}

\subsubsection{Definições}

Nesta seção vamos apresentar a classe de distribuições mistura de escala da normal introduzida por Andrews \& Mallows (1974), uma extensão paramétrica robusta do modelo normal que preserva a estrutura simétrica e possibilita reduzir a influência dos outliers. 
Começaremos definindo as distribuições mistura de escala da normal através de sua representação hierárquica, para logo em seguida apresentar alguns exemplos de distribuições que pertencem a esta classe.

Definição 1. Dizemos que uma variável aleatória $X$ tem uma distribuição mistura de escala da normal, com parâmetro de locação $\mu$ e parâmetro de dispersão $\sigma^{2}>0$, se tem a seguinte representação estocástica:

$$
X=\mu+U^{-\frac{1}{2}} Z, \quad Z \perp U
$$

em que $Z \sim \mathrm{N}\left(0, \sigma^{2}\right)$, U é uma variável aleatória positiva com fda $H(\cdot \mid \boldsymbol{\nu})$ e $\boldsymbol{\nu}$ é o parâmetro (ou vetor de parâmetros) indexado à distribuição de $U$.

Cometendo um pequeno abuso de notação, escreveremos $X \sim \operatorname{SMN}\left(\mu, \sigma^{2}, \boldsymbol{\nu}\right)$ (Scale Mixture of Normal). Quando $\mu=0$ e $\sigma^{2}=1$ temos a distribuição SMN padronizada. Note de (1.1) que $X \mid U=u \sim N\left(\mu, u^{-1} \sigma^{2}\right)$. Assim, integrando em $U$ a densidade conjunta de $X$ e $U$, obtemos a densidade marginal de $X$ :

$$
f_{S M N}\left(x \mid \mu, \sigma^{2}, \boldsymbol{\nu}\right)=\left(2 \pi \sigma^{2}\right)^{-\frac{1}{2}} \int_{0}^{\infty} u^{\frac{1}{2}} \exp \left\{-\left(u / 2 \sigma^{2}\right)(x-\mu)^{2}\right\} d H(u \mid \boldsymbol{\nu}),
$$

em que $U$ é chamado de fator de escala e $H(\cdot \mid \boldsymbol{\nu})$ é chamada de distribuição de mistura. Dependendo da distribuição do fator de escala $U$, temos uma distribuição em particular da classe da família SMN.

É importante notar que existe uma relação entre as distribuições SMN e as distribuições elípticas. Dizemos que a variável aleatória $X$ tem uma distribuição elíptica univariada, com parâmetro de locação $\mu$ e parâmetro de dispersão $\sigma^{2}$, quando a sua densidade é dada por

$$
f(x)=\sigma^{-1} g(z),
$$

em que $z=(x-\mu)^{2} / \sigma^{2}$ e $g: \mathbb{R} \rightarrow[0, \infty)$ satisfaz $\int_{0}^{\infty} z^{-\frac{1}{2}} g(z) d z<\infty$. É fácil notar que (1.2) tem a forma (1.3). A relação entre as distribuições SMN e elípticas serão utilizadas na Seção 2.5 para obter o erro padrão para os parâmetros da regressão.

Definição 2. Seja $X \sim S M N\left(\mu, \sigma^{2}, \nu\right)$ e $a<b$, tal que $P(a<X<b)>0$. A variável aleatória $Y$ tem uma distribuição $S M N$ truncada no intervalo, se esta tem a mesma distribuição que $X \mid X \in(a, b)$. Neste caso, escrevemos $Y \sim \operatorname{TSMN}_{(a, b)}\left(\mu, \sigma^{2}, \boldsymbol{\nu}\right)$.

Como uma consequência obvia da Definição 2, podemos obter a densidade de $Y \sim$ $\operatorname{TSMN}_{(a, b)}\left(\mu, \sigma^{2}, \boldsymbol{\nu}\right)$, dada por

$$
\begin{aligned}
& f_{\operatorname{TSMN}}\left(y \mid \mu, \sigma^{2}, \boldsymbol{\nu} ;(a, b)\right)= \\
& f_{S M N}\left(y \mid \mu, \sigma^{2}, \boldsymbol{\nu}\right)\left[F_{S M N}\left(\frac{b-\mu}{\sigma}\right)-F_{S M N}\left(\frac{a-\mu}{\sigma}\right)\right]^{-1}, a<y<b,
\end{aligned}
$$

e $f_{\operatorname{TSMN}}\left(y \mid \mu, \sigma^{2}, \boldsymbol{\nu} ;(a, b)\right)=0$ em outro caso, em que $\mathcal{F}_{S M N}(\cdot)$ denota a fda da distribuição SMN padronizada. 


\subsubsection{Exemplo de distribuições SMN}

O fator de escala $U$ apresentado em (1.1) é uma variável aleatória positiva, que pode ser discreta ou contínua. A forma da distribuição SMN é determinada por $U$. Assim temos que:

- Distribuição Normal: neste caso o fator de escala $U$ é uma variável aleatória degenerada em 1, isto é, $P(U=1)=1$.

- Distribuição Pearson tipo VII: neste caso $U \sim \operatorname{Gamma}(\nu / 2, \delta / 2), \operatorname{com} \nu>0$ e $\delta>$ 0, em que $\operatorname{Gamma}(a, b)$ denota a distribuição Gama com média $a / b$. A fdp da variável aleatória $X$, definida em (1.1), toma a forma

$$
f_{P V I I}(x \mid \nu, \delta)=\frac{1}{B(\nu / 2,1 / 2) \sqrt{\delta}}\left(1+\frac{x^{2}}{\delta}\right)^{-\frac{\nu+1}{2}},
$$

em que $\delta>0$ e $\nu>0$ são parâmetros de forma e $B(a, b)$ representa a função beta. Utilizamos a notação $X \sim P V I I(0,1 ; \nu, \delta)$.

A fda da distribuição Pearson tipo VII é dada por

$$
F_{P V I I}(x)=\frac{1}{2}+\frac{y}{B(\nu / 2,1 / 2) \sqrt{\delta+x^{2}}} 2 F_{1}\left(\frac{1}{2}, 1-\frac{\nu}{2} ; \frac{3}{2} ; \frac{x^{2}}{\delta+x^{2}}\right),
$$

em que ${ }_{2} F_{1}$ é uma função hipergeométrica Gauss definida pela série

$$
{ }_{2} F_{1}(a, b ; c, d)=\sum_{k=0}^{\infty} \frac{(a)_{k}(b)_{k}}{(c)_{k}} \frac{x^{k}}{k !},
$$

em que $(z)_{k}=z(z+1) \ldots(z+k-1)$ denota o fatorial ascendente.

Quando $\delta=\nu$ temos a distribuição t de Student $\operatorname{com} \nu$ graus de liberdade. Assim também, quando $\delta=\nu=1$ temos a distribuição Cauchy.

- Distribuição Slash: aqui a distribuição do fator de escala $U$ é $\operatorname{Beta}(\nu, 1), \operatorname{com} \nu>0$. A densidade da variável aleatória $X$, definida em (1.1), é dada por

$$
f_{S L}(x \mid \nu)=\nu \int_{0}^{1} u^{\nu-1} \phi\left(x u^{\frac{1}{2}}\right) d u, \quad-\infty<x<\infty .
$$

Utilizamos a notação $X \sim S L(0,1 ; \nu)$. A fda da distribuição Slash é dada por

$$
\begin{aligned}
F_{S L}(x) & =\nu \int_{0}^{1} u^{\nu-1} \Phi(x \sqrt{u}) d u \\
& =\frac{1}{2}+\frac{\nu y}{\sqrt{2 \pi}} \int_{0}^{1} u^{\nu-1 / 2}{ }_{1} F_{1}\left(\frac{1}{2} ; \frac{3}{2} ;-\frac{u x^{2}}{2}\right) d u,
\end{aligned}
$$

em que ${ }_{1} F_{1}$ é uma função hipergeométrica Gauss definida pela série

$$
{ }_{1} F_{1}(a ; b ; x)=\sum_{k=0}^{\infty} \frac{(a)_{k}}{(b)_{k}} \frac{x^{k}}{k !},
$$

em que $(z)_{k}$ denota o fatorial ascendente definida anteriormente. 
- Distribuição normal contaminada: neste caso $U$ é uma variável aleatória discreta que pode assumir dois valores: 1 ou $\gamma$. Neste caso, a função de probabilidade de $U$ é dada por

$$
U=\left\{\begin{array}{cc}
\gamma & \text { com probabilidade } \nu ; \\
1 & \text { com probabilidade } 1-\nu,
\end{array}\right.
$$

Segue imediatamente que a densidade da variável aleatória $X$, definida em (1.1), é dada por

$$
f_{C N}(x \mid \nu, \gamma)=\nu \phi\left(x \mid 0, \gamma^{-1}\right)+(1-\nu) \phi(x)
$$

A fda da distribuição normal contaminada é dada por

$$
F_{C N}(x)=\nu \Phi\left(x \mid 0, \gamma^{-1}\right)+(1-\nu) \Phi(x) .
$$

Na Figura 1.1 apresentamos os gráficos das densidades das distribuições da classe SMN, com média $\mu=0$ e variância igual 1: Normal, t de Student com $\nu=5$, slash com $\nu=3 \mathrm{e}$ normal contaminada $\operatorname{com} \boldsymbol{\nu}=(0.5,0.5)$
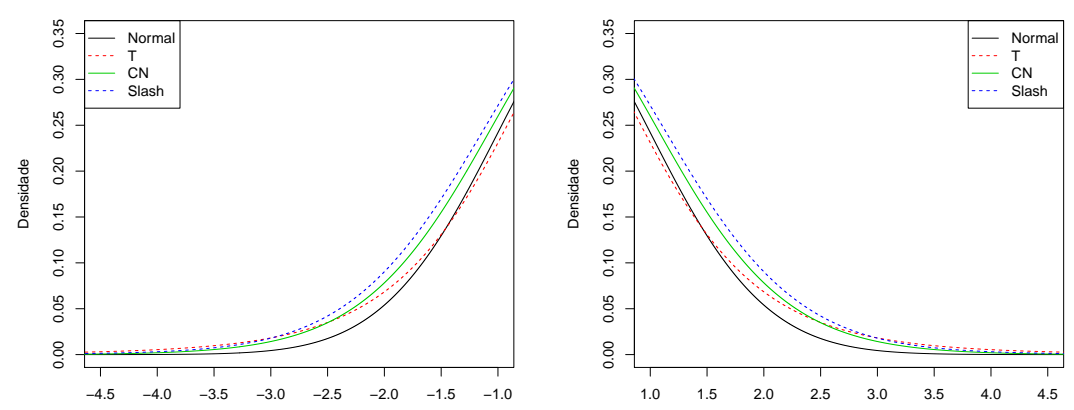

Figura 1.1: Densidade da classe de distribuições SMN.

A proposição apresentada a seguir, a qual é uma extensão natural do Teorema 1 (e Corolário 1) de Genç (2013), é crucial para o desenvolvimento de nossa proposta teórica. No que segue, $\mathrm{E}[\cdot]$ denota esperança, $\mathrm{E}_{X}[\cdot]$ denota esperança relativa à distribuição de $X$ e, por simplicidade, denotaremos todas as fdp por $f(\cdot)$. Assim, por exemplo, $f(u, x)$ denota a fdp conjunta de $U$ e $X$, e $f(u \mid X \in \mathcal{A})$ denota a fdp de $U$ dado o evento $\{X \in \mathcal{A}\}$. 
Proposição 1. Seja $X \sim \operatorname{SMN}(0,1, \boldsymbol{\nu})$ com fator de escala $U$ e distribuição de mistura $H(\cdot \mid \boldsymbol{\nu})$. Então, para $a<b, a \mathrm{E}\left[U^{r} X^{s} \mid X \in(a, b)\right]$ para $r \geq 1$ es $s=0,1,2$ é dada por:

$$
\begin{aligned}
\mathrm{E}\left[U^{r} \mid X \in(a, b)\right]= & \tau(a, b) \times\left[\mathrm{E}_{\Phi}(r, b)-\mathrm{E}_{\Phi}(r, a)\right] \\
\mathrm{E}\left[U^{r} X \mid X \in(a, b)\right]= & \tau(a, b) \times\left[\mathrm{E}_{\phi}\left(r-\frac{1}{2}, a\right)-\mathrm{E}_{\phi}\left(r-\frac{1}{2}, b\right)\right] ; \\
\mathrm{E}\left[U^{r} X^{2} \mid X \in(a, b)\right]= & \tau(a, b) \times\left[\mathrm{E}_{\Phi}(r-1, b)\right. \\
& \left.-\mathrm{E}_{\Phi}(r-1, a)+a \mathrm{E}_{\phi}\left(r-\frac{1}{2}, a\right)-b \mathrm{E}_{\phi}\left(r-\frac{1}{2}, b\right)\right],
\end{aligned}
$$

em que

$$
\begin{aligned}
\tau(a, b) & =\left(F_{S M N}(b)-F_{S M N}(a)\right)^{-1} ; \\
\mathrm{E}_{\phi}(r, h) & =\mathrm{E}\left[U^{r} \phi\left(h U^{\frac{1}{2}}\right)\right]=\int_{0}^{\infty} u^{r} \phi\left(h u^{\frac{1}{2}}\right) d H(u \mid \boldsymbol{\nu}) ; \\
\mathrm{E}_{\Phi}(r, h) & =\mathrm{E}\left[U^{r} \Phi\left(h U^{\frac{1}{2}}\right)\right]=\int_{0}^{\infty} u^{r} \Phi\left(h u^{\frac{1}{2}}\right) d H(u \mid \boldsymbol{\nu}) .
\end{aligned}
$$

Demonstração. Seja $\mathcal{A}=(a, b)$. Das Definições 1 e 2 , temos que $X \mid U=u \sim \mathrm{N}\left(0, u^{-1}\right)$, $X \mid X \in \mathcal{A} \sim \operatorname{TSMN}_{\mathcal{A}}(0,1, \boldsymbol{\nu})$ e $X \mid U=u, X \in \mathcal{A} \sim \operatorname{TN}_{\mathcal{A}}\left(0, u^{-1}\right)$, que é, a distribuição Normal truncada em $\mathcal{A}$, com média 0 e variância $u^{-1}$, antes do truncamento. Então, para $r \geq 1$ e $s=0,1,2$ temos que:

$$
\begin{aligned}
\mathrm{E}\left[U^{r} X^{s} \mid X \in \mathcal{A}\right] & =\mathrm{E}_{U}\left[U^{r} \mathrm{E}_{X}\left[X^{s} \mid U, X \in \mathcal{A}\right] \mid X \in \mathcal{A}\right] \\
& =\int_{0}^{\infty} U^{r} \mathrm{E}_{X}\left[X^{s} \mid U, X \in \mathcal{A}\right] f(u \mid X \in \mathcal{A}) d u
\end{aligned}
$$

A fdp na equação (1.8) assume a seguinte forma:

$$
\begin{aligned}
f(u \mid X \in \mathcal{A}) & =\int f(u, x \mid X \in \mathcal{A}) d x \\
& =\int f(u \mid X=x, X \in \mathcal{A}) f(x \mid X \in \mathcal{A}) d x \\
& =\tau(a, b) \int f(u \mid X=x, X \in \mathcal{A}) f(x) \mathbb{I}_{\mathcal{A}}(x) d x \\
& =\tau(a, b) \int f(u, x) \mathbb{I}_{\mathcal{A}}(x) d x \\
& =\tau(a, b) \int_{\mathcal{A}} f(u) \phi\left(x \mid 0, u^{-1}\right) d x \\
& =\tau(a, b) f(u) \int_{\mathcal{A}^{*}} \phi(z) d z \\
& =\tau(a, b) f(u)\left[\Phi\left(b u^{\frac{1}{2}}\right)-\Phi\left(a u^{\frac{1}{2}}\right)\right],
\end{aligned}
$$

em que $\mathcal{A}^{*}=\left(a u^{\frac{1}{2}}, b u^{\frac{1}{2}}\right)$. A Equação (1.10) é obtida utilizando as fdp de $X \mid X \in \mathcal{A}$. A Equação (1.11) é consequência de que, se $x \in \mathcal{A}$, então $\{X \in \mathcal{A}, X=x\}=\{X=x\}$, implicando que $f(u, x)=f(u \mid X=x) f(x)=f(u \mid X=x, X \in \mathcal{A}) f(x)$. Se $x \notin \mathcal{A}$, então $\mathbb{I}_{\mathcal{A}}(x)=0$ e as integrais em (1.10) e (1.11) são iguais a zero. Por (1.8) e pelo Lema 1 , dado no Apêndice A, segue que: 
- $\operatorname{para} s=0$,

$$
\begin{aligned}
\mathrm{E}\left[U^{r} \mid X \in \mathcal{A}\right] & =\int_{0}^{\infty} u^{r} f(u \mid X \in \mathcal{A}) d u \\
& =\tau(a, b) \mathrm{E}_{U}\left\{U^{r}\left[\Phi\left(b U^{\frac{1}{2}}\right)-\Phi\left(a U^{\frac{1}{2}}\right)\right]\right\}
\end{aligned}
$$

- $\operatorname{para} s=1$,

$$
\begin{aligned}
\mathrm{E}\left[U^{r} X \mid X \in \mathcal{A}\right] & =\int_{0}^{\infty} \frac{u^{r}}{u^{\frac{1}{2}}} \frac{\phi\left(a u^{\frac{1}{2}}\right)-\phi\left(b u^{\frac{1}{2}}\right)}{\Phi\left(b u^{\frac{1}{2}}\right)-\Phi\left(a u^{\frac{1}{2}}\right)} f(u \mid X \in \mathcal{A})(u) d u \\
& =\tau(a, b) \mathrm{E}_{U}\left\{U^{r-\frac{1}{2}}\left[\phi\left(a U^{\frac{1}{2}}\right)-\phi\left(b U^{\frac{1}{2}}\right)\right]\right\}
\end{aligned}
$$

- $\operatorname{para} s=2$,

$$
\begin{aligned}
\mathrm{E}\left[U^{r} X^{2} \mid X \in \mathcal{A}\right] & =\int_{0}^{\infty}\left[u^{r-1}+\frac{a u^{r-\frac{1}{2}} \phi\left(a u^{\frac{1}{2}}\right)-b u^{r-\frac{1}{2}} \phi\left(b u^{\frac{1}{2}}\right)}{\Phi\left(b u^{\frac{1}{2}}\right)-\Phi\left(a u^{\frac{1}{2}}\right)}\right] f(u \mid X \in \mathcal{A}) d u \\
& =\tau(a, b) \mathrm{E}_{U}\left\{U^{r-1}\left[\Phi\left(b U^{\frac{1}{2}}\right)-\Phi\left(a U^{\frac{1}{2}}\right)\right]\right. \\
& \left.+U^{r-\frac{1}{2}}\left[a \phi\left(a U^{\frac{1}{2}}\right)-b \phi\left(b U^{\frac{1}{2}}\right)\right]\right\} .
\end{aligned}
$$

Quando a distribuição de $U$ for definida, esta proposição apresenta expressões fechadas para os valores esperados $\mathrm{E}\left[U^{r} X^{s} \mid X \in(a, b)\right]$, em que $s=0,1,2$ e $r \geq 1$.

Agora, calcularemos as quantidades $E_{\phi}(r, h)$ e $E_{\Phi}(r, h)$ para algumas distribuições da família SMN. Estes resultados são úteis para a implementação do algoritmo EM.

- Distribuição Pearson tipo VII: Neste caso, temos que

$$
\begin{aligned}
& \mathrm{E}_{\Phi}(r, h)=\frac{\Gamma\left(\frac{\nu+2 r}{2}\right)}{\Gamma\left(\frac{\nu}{2}\right)}\left(\frac{\delta}{2}\right)^{-r} F_{P V I I}(h \mid \nu+2 r, \delta) ; \\
& \mathrm{E}_{\phi}(r, h)=\frac{\Gamma\left(\frac{\nu+2 r}{2}\right)}{\Gamma\left(\frac{\nu}{2}\right) \sqrt{2 \pi}}\left(\frac{\delta}{2}\right)^{\frac{\nu}{2}}\left(\frac{h^{2}+\delta}{2}\right)^{-\frac{(\nu+2 r)}{2}},
\end{aligned}
$$

em que $\Gamma(a)$ é a função gama e $F_{P V I I}(\cdot)$ é a fda da distribuição Pearson tipo VII.

- Distribuição Slash: Temos que

$$
\begin{aligned}
& \mathrm{E}_{\Phi}(r, h)=\left(\frac{\nu}{\nu+r}\right) F_{S L}(h \mid \nu+r) ; \\
& \mathrm{E}_{\phi}(r, h)=\frac{\nu}{\sqrt{2 \pi}}\left(\frac{h^{2}}{2}\right)^{-(\nu+r)} \Gamma\left(\nu+r, \frac{h^{2}}{2}\right),
\end{aligned}
$$

em que $\Gamma(a, b)=\int_{0}^{b} e^{-t} t^{a-1} d t$ é a função gama incompleta e $F_{S L}(\cdot)$ é a fda da distribuição Slash. Para maiores detalhes, veja Lema 6 em Genç (2013). 
- Distribuição normal contaminada: Neste caso temos que

$$
\begin{aligned}
& \mathrm{E}_{\Phi}(r, h)=\gamma^{r} F_{C N}(h \mid \nu, \gamma)+\left(1-\gamma^{r}\right)(1-\nu) \Phi(h) ; \\
& \mathrm{E}_{\phi}(r, h)=\nu \gamma^{r} \phi(h \sqrt{\gamma})+(1-\nu) \phi(h),
\end{aligned}
$$

em que $F_{C N}(\cdot)$ é a fda da distribuição normal contaminada.

Como uma direta consequência da Proposição 1, no Apêndice A apresentamos alguns Corolários importantes, os quais serão utilizados para a implementação do algoritmo EM.

Tendo como base o que foi discutido até aqui, apresentaremos uma breve descrição do algoritmo EM, que será utilizado nos próximos Capítulos para encontrar as estimativas de máxima verossimilhança (MV) dos parâmetros nos modelos lineares censurados sob a classe de distribuições SMN. Também apresentaremos um resumo dos métodos de simulação estocástica, que usaremos para avaliar nosso modelo a partir de uma perspectiva Bayesiana.

\subsection{Algoritmo EM}

O Algoritmo EM (Expectation-Maximization) introduzido por Dempster et al. (1977), é uma ferramenta desenvolvida para obter as estimativas dos parâmetros $\boldsymbol{\theta}$ do modelo através do cálculo iterativo pelo método de máxima verossimilhança. A técnica consiste basicamente em considerar uma representação do modelo em "dados aumentados", que nada mais é do que uma representação estocástica em termos de distribuições mais tratáveis, que em geral dependem de quantidades não observáveis $\mathbf{y}_{n o b s}$, que chamamos de "dados perdidos". A ideia do procedimento é então obter uma log-verossimilhança aumentada - chamada de log-verossimilhança completa, denotada por por $\ell_{c}(\boldsymbol{\theta} \mid \mathbf{z})$, em que $\mathbf{z}=\left(\mathbf{y}_{\text {obs }}, \mathbf{y}_{\text {nobs }}\right)$ representa os dados aumentados, ou seja, supondo que as quantidades não observáveis foram de fato observadas.

Cada iteração do algoritmo EM consiste em dois passos:

\section{- Passo E (Expectation):}

Este passo consiste em calcular a esperança da log-verossimilhança completa, denotada por $Q\left(\boldsymbol{\theta} \mid \widehat{\boldsymbol{\theta}}^{(k-1)}\right)$, condicionada ao vetor de dados observados. Isto é, para a $k$-ésima iteração temos que, dado $\widehat{\boldsymbol{\theta}}=\widehat{\boldsymbol{\theta}}^{(k-1)}$,

$$
Q\left(\boldsymbol{\theta} \mid \widehat{\boldsymbol{\theta}}^{(k-1)}\right)=E\left[\ell_{c}(\boldsymbol{\theta} \mid \mathbf{z}) \mid \mathbf{y}_{o b s}, \widehat{\boldsymbol{\theta}}^{(k-1)}\right] .
$$

\section{- Passo M (Maximization):}

Consiste em maximizar a esperança da log-verossimilhança completa em relação aos parâmetros do modelo, substituindo os "dados perdidos" por seus valores esperados condicionais, obtidos no passo E. Para a $k$-ésima iteração obtemos $\widehat{\boldsymbol{\theta}}^{(k)}$ que maximize $Q\left(\boldsymbol{\theta} \mid \widehat{\boldsymbol{\theta}}^{(k-1)}\right)$, tal que

$$
Q\left(\widehat{\boldsymbol{\theta}}^{(k)} \mid \widehat{\boldsymbol{\theta}}^{(k-1)}\right)>Q\left(\boldsymbol{\theta} \mid \widehat{\boldsymbol{\theta}}^{(k-1)}\right), \quad \forall \boldsymbol{\theta} \in \boldsymbol{\Theta}
$$


Em geral, a maximização simultânea de todas as componentes do vetor $\boldsymbol{\theta}$ é um problema extremamente difícil, do ponto de vista numérico. Uma alternativa eficiente é maximizar as coordenadas marginalmente. Este procedimento é denominado maximização condicional (CM), e o algoritmo resultante é conhecido como ECM (Meng \& Rubin, 1993). No entanto, em alguns casos, como nos modelos que desenvolveremos posteriormente, é conveniente usar uma extensão do algoritmo EM e ECM, conhecido como Algoritmo ECME (Liu \& Rubin, 1994), que é obtido maximizando a correspondente função de verossimilhança marginal restrita.

Assim, este processo é iterado até que uma regra de convergência conveniente como $\left\|\widehat{\boldsymbol{\theta}}^{(k)}-\widehat{\boldsymbol{\theta}}^{(k-1)}\right\|$, ou uma função da diferença envolvendo duas avaliações sucessivas da logverossimilhança $\ell(\cdot \mid \mathbf{y})$, seja suficientemente pequena.

\subsection{Estimação Bayesiana}

Seja $\mathbf{y}=\left(y_{1}, \ldots, y_{n}\right)$ uma amostra aleatória observada de uma variável aleatória $Y$, com densidade (ou função de probabilidade, se $Y$ for discreta) $f(\cdot \mid \boldsymbol{\theta})$. O objetivo é desenvolver inferência Bayesiana para o vetor de parâmetros $\boldsymbol{\theta} \in \boldsymbol{\Theta}$, em que $\boldsymbol{\Theta}$ é o espaço paramétrico. O modelo Bayesiano é definido pela função de verossimilhança

$$
L(\boldsymbol{\theta} \mid \mathbf{y})=f\left(y_{1} \mid \boldsymbol{\theta}\right) \cdots f\left(y_{n} \mid \boldsymbol{\theta}\right), \quad \boldsymbol{\theta} \in \mathbf{\Theta},
$$

em conjunto com uma distribuição a priori $\pi(\boldsymbol{\theta})$, representando informação anterior ou crença pessoal em relação à distribuição de probabilidade de $\boldsymbol{\theta}$. A densidade a posteriori (ou posterior como preferem alguns) de $\boldsymbol{\theta} \mid \mathbf{y}$ é dada por

$$
\pi(\boldsymbol{\theta} \mid \mathbf{y})=\frac{L(\boldsymbol{\theta} \mid \mathbf{y}) \pi(\boldsymbol{\theta})}{h(\mathbf{y})}, \quad \boldsymbol{\theta} \in \boldsymbol{\Theta}
$$

em que $h(\mathbf{y})$ é a densidade marginal de $\mathbf{y}$.

Em muitas aplicações não necessitamos da constante $h(\mathbf{y})$ e escrevemos simplesmente

$$
\pi(\boldsymbol{\theta} \mid \mathbf{y}) \propto L(\boldsymbol{\theta} \mid \mathbf{y}) \pi(\boldsymbol{\theta})
$$

Dizemos que a expressão acima é a forma não normalizada de $\pi(\boldsymbol{\theta} \mid \mathbf{y})$. No entanto, caso seja necessário, uma forma de obter a constante normalizadora é resolver a integral

$$
h(\mathbf{y})=\int \pi(\mathbf{y}, \boldsymbol{\theta}) d \boldsymbol{\theta}=\int L(\boldsymbol{\theta} \mid \mathbf{y}) \pi(\boldsymbol{\theta}) d \boldsymbol{\theta},
$$

em que $\pi(\mathbf{y}, \boldsymbol{\theta})$ é a densidade conjunta de $\mathbf{y}$ e $\boldsymbol{\theta}$ (em um abuso de notação, utilizaremos a notação $\pi(\cdot)$ para qualquer densidade. $\mathrm{O}$ argumento entre parênteses indicará a variável correspondente).

É importante enfatizar que, no modelo que acabamos de definir, as observações $Y_{1}, \ldots, Y_{n}$ são independentes condicionadas ao conhecimento do valor do parâmetro $\boldsymbol{\theta}$. Marginalmente, 
é plenamente possível que elas não sejam independentes, ou seja, que a densidade $h(\mathbf{y})$ não seja o produto das densidades marginais de $Y_{i}, i=1, \ldots, n$, dadas por

$$
h\left(y_{i}\right)=\int f\left(y_{i} \mid \boldsymbol{\theta}\right) \pi(\boldsymbol{\theta}) d \boldsymbol{\theta} .
$$

As estimativas no contexto Bayesiano são obtidas a partir de características da distribuição a posteriori, como a média, moda, etc. Por exemplo, temos que uma estimativa para $\theta$ é

$$
\widehat{\boldsymbol{\theta}}=E[\boldsymbol{\theta} \mid \mathbf{y}]=\int \boldsymbol{\theta} \pi(\boldsymbol{\theta} \mid \mathbf{y}) d \boldsymbol{\theta} .
$$

Ao longo do texto, por simplicidade, uma distribuição de probabilidade a priori e uma função de densidade de probabilidade a priori, serão chamadas de distribuição a priori e densidade a priori (analogamente para o caso da posteriori). O fator de normalização $h(\mathbf{y})$ é fundamental para a implementação do método Bayesiano.

Nas duas últimas décadas, a inferência Bayesiana tem apresentado um grande avanço devido à introdução de métodos numéricos de computação intensiva que permitem, de forma relativamente simples, obter amostras da distribuição objetivo. Em particular, os métodos numéricos do tipo Monte Carlo, via Cadeias de Markov (MCMC) (Gamerman \& Lopes, 2006) tem recebido grande atenção. Existem vários métodos propostos para construir uma cadeia de Markov, nas subseções 1.4.1 e 1.4.2 discutimos os dois mais conhecidos, que serão utilizados no Capítulo 3.

\subsubsection{Amostrador de Gibbs}

O amostrador de Gibss, proposto por Geman \& Geman (1984) e adaptado com sucesso para a comunidade estatística por Gelfand et al. (1990), é talvez o método MCMC mais disseminado na área Bayesiana . Este algoritmo permite obter uma amostra da distribuição a posteriori sem conhecimento de sua constante normalizadora.

A ideia por trás deste algoritmo é criar uma cadeia de Markov $\left\{\theta_{(1)}, \theta_{(2)}, \ldots\right\}$, que tenha distribuição estacionária $\pi(\theta \mid \mathbf{y})$. Informalmente, isto significa que é possível especificar o que chamamos de núcleo de transição da cadeia, dado por

$$
P\left(\theta_{(i+1)} \in A \mid \theta_{(i)}=\theta_{(i)}^{*}\right), \quad \text { para todo } A,
$$

em que $\theta_{(i)}^{*}$ é um ponto fixo do espaço paramétrico, de modo que, para todo $A$ :

$$
P\left(\theta_{(i+1)} \in A \mid \theta_{(i)}=\theta_{(i)}^{*}\right) \longrightarrow \int_{A} \pi(\theta \mid \mathbf{y}) d \theta \quad \text { conforme } \quad i \rightarrow \infty
$$

Ou seja, dado que no estágio $i$ a cadeia toma o valor $\theta_{(i)}^{*}$, sabemos a probablilidade dela estar em $A$, no estágio $i+1$. Suponha que $\pi_{0}(\cdot)$ é uma distribuição que fornece valores iniciais para a cadeia. Digamos que temos um valor inicial $\theta_{(0)}^{*}$ gerado a partir de $\pi_{0}(\cdot)$, então podemos obter $P\left(\theta_{(1)} \in A \mid \theta_{(0)}=\theta_{(0)}^{*}\right)$. Conhecendo esta distribuição condicional, podemos gerar uma observação a partir da mesma, e obter $\theta_{(1)}^{*}$. Como conhecemos o núcleo, podemos 
obter a distribuição $P\left(\theta_{(2)} \in A \mid \theta_{(1)}=\theta_{1}^{*}\right)$. Este processo pode ser estendido indefinidamente pela propriedade básica das cadeias de Markov, ou seja

$$
P\left(\theta_{(i+1)} \in A \mid \theta_{(1)}=\theta_{(1)}^{*}, \ldots, \theta_{(i)}=\theta_{(i)}^{*}\right)=P\left(\theta_{(i+1)} \in A \mid \theta_{(i)}=\theta_{(i)}^{*}\right),
$$

(isto é, dado o presente, o futuro é independente do passado). Neste caso, para grandes valores de $i$, é esperado que a distribuição de $\theta_{(i)}$ seja aproximadamente a distribuição a posteriori $\pi(\theta \mid \mathbf{y})$.

Algumas referências adicionais com propriedades do amostrador de Gibbs e citações, envolvendo resultados teóricos mais gerais em torno de algoritmos do tipo MCMC, podem ser encontradas também nos trabalhos de Casella \& George (1992) e Gelfand (1995).

\subsubsection{O algoritmo Metropolis-Hastings}

Este algoritmo, inicialmente proposto por Metropolis et al. (1953) e estendido por Hastings (1970), utiliza uma distribuição auxiliar para selecionar um valor e propô-lo como uma nova observação para a cadeia. Esse valor será aceito com uma probabilidade $\alpha$.

Assim, vamos assumir que queremos gerar valores de uma distribuição $\pi(\theta \mid \cdots)$, em que $\theta$ é um escalar, e seja $q(\theta \mid \cdots)$ um núcleo de transição arbitrário de em que é fácil gerar valores para $\theta$.

Suponha então, que na iteração $i$ o estado da cadeia é $\theta^{(i)}$. A posição na iteração $i+1$ será denotada por $\theta^{(i+1)}$, e será obtida da seguinte forma:

- Gere uma observação $\theta^{*}$ a partir de $q\left(\theta_{(i)} \mid \cdots\right)$;

- Aceite a observação proposta com probabilidade

$$
\alpha\left(\theta^{(j)}, \theta^{*}\right)=\min \left\{\frac{\pi\left(\theta^{*} \mid \cdots\right) q\left(\theta^{*} \mid \theta^{(i)}\right)}{\pi\left(\theta^{(i)} \mid \cdots\right) q\left(\theta^{(i)} \mid \theta^{*}\right)}, 1\right\}, \text { fazendo } \theta^{i+1}=\theta^{*},
$$

ou rejeite a observação gerada com probabilidade $1-\alpha\left(\theta^{(j)}, \theta^{*}\right)$ fazendo $\theta^{i+1}=\theta^{(j)}$.

Em muitos casos construímos um amostrador de Gibbs no qual é necessário gerar observações de condicionais completas que não possuem forma analítica fechada. Nestes casos, podemos utilizar o algoritmo de Metropolis-Hastings para gerar tais observações e dizemos que estamos utilizando o algoritmo amostrador de Gibbs, com passos de MetropolisHastings. Veja Cabral et al. (2012) para mais detalhes sobre este algoritmo.

No seguinte Capítulo, abordaremos as modelos lineares censurados sob a classe de distribuições SMN a partir de um enfoque clássico, utilizando as ferramentas e conceitos descritos neste Capítulo. 


\section{Capítulo 2}

\section{Modelos lineares censurados sob a classe de distribuições SMN}

\subsection{Introdução}

Modelos de regressão lineares cuja variável resposta é censurada (denotados por CR) são aplicados em muitas áreas do conhecimento, tais como econometria, ensaios clínicos, pesquisas médicas, estudos de engenharia, entre outros.

Em econometria, por exemplo, o estudo da participação da força de trabalho das mulheres casadas é realizado rotineiramente considerando o modelo Tobit (Greene, 2012) ou algum outro modelo alternativo (Martinez-Florez et al., 2013). Neste caso, a resposta observada é a taxa de salários, que é tipicamente considerada como censurada abaixo de zero, isto é, para as mulheres que trabalham, são registrados valores positivos para os salários, enquanto que, para as mulheres que não trabalham, os salários observados assumem valor zero (veja Mroz, 1987).

Já na pesquisa sobre HIV, as medidas de carga viral podem ser submetidas a alguns limites de detecção superiores e inferiores, abaixo ou acima do qual elas não são quantificáveis. Como resultado, as respostas de carga viral são censuradas à esquerda ou à direita, dependendo dos ensaios de diagnóstico usados (veja $\mathrm{Wu}, 2010$ ).

Em geral, por razões matemáticas, é assumido que os erros aleatórios seguem distribuição normal (Wei \& Tanner, 1990). No entanto, esta suposição pode ser não realista em diversas situações, comprometendo seriamente os resultados inferenciais.

Do ponto de vista prático, talvez o método mais utilizado para aproximar normalidade com razoáveis resultados empíricos seja a transformação de variáveis (como por exemplo Box-Cox, etc). No entanto, algumas das possíveis desvantagens destes métodos são: (i) A transformação não fornece informação útil para entender o mecanismo; (ii) A transformação de variáveis dificulta a interpretação, especialmente quando temos diversas variáveis de interesse e cada uma é transformada utilizando diferentes funções; (iii) As transformações podem não ser universais e pode frequentemente não ser aplicável para outros conjunto de dados. Assim, a partir de uma perspectiva prática, há necessidade de buscar modelos 
alternativos ao normal, que preservem a estrutura simétrica e que possibilitem reduzir a influência dos outliers.

Para lidar com o problema de observações atípicas em modelos de regressão com respostas completas, foram propostas na literatura classes de distribuições mais flexíveis que a distribuição normal. Por exemplo, Lange et al. (1989) discutiram a utilização da distribuição t de Student em modelos de regressão múltipla. Neste caso, os graus de liberdade é o parâmetro de escolha natural para controlar a curtose. Ibacache-Pulgar \& Paula (2011) propuseram algumas medidas de influência local em modelos t de Student parcialmente lineares. Villegas et al. (2012) propuseram os modelos lineares generalizados simétricos, em que a função de ligação é definida para estabelecer uma relação entre os valores médios das distribuições simétricas e os preditores lineares. Arellano-Valle et al. (2012) utilizaram a distribuição t de Student no contexto de modelos de regressão censurada. Massuia et al. (2012) desenvolveram medidas de diagnóstico para este modelo, incluindo a implementação de um interessante (e simples) algoritmo EM para encontrar as estimativas de MV.

Embora existam algumas propostas que permitam superar o problema da presença de observações atípicas nos modelos CR, não existem estudos, que considerem ao mesmo tempo, a variável resposta censurada e erros de observação modelados sob a classe de distribuições misturas da escala normal (SMN), o que é, talvez, a mais importante da família das distribuições simétricas. As distribuições SMN são extensões da distribuição normal incorporando curtose. As distribuições t de Student, Pearson tipo VII (PVII), slash (SL), exponencial potência $(\mathrm{PE})$, normal contaminada $(\mathrm{CN})$ e, obviamente a distribuição normal $(\mathrm{N})$ estão incluídas nesta classe. Pesquisas abrangentes estão disponíveis em Fang \& Zhang (1990), Arellano-Valle (1994) e Meza et al. (2012), entre outros.

Neste texto apresentaremos os modelos lineares censurados sob a classe de distribuições SMN, daqui em diante denotado por SMN-CR, enfatizando procedimentos para estimação de máxima verossimilhança e cálculo dos erros padrões assintóticos dos parâmetros destes modelos. Os resultados deste capítulo servirão como base para o estudo dos modelos lineares censurados sob a classe de distribuições SMN a partir de uma perspectiva Bayesiana.

\subsection{Descrição do modelo}

Consideremos inicialmente o modelo linear sob a classe de distribuições SMN definido por:

$$
Y_{i}=\mathbf{x}_{i}^{\top} \boldsymbol{\beta}+\varepsilon_{i}, \quad \varepsilon_{i} \stackrel{\text { iid }}{\sim} \operatorname{SMN}\left(0, \sigma^{2}, \boldsymbol{\nu}\right), \quad i=1, \ldots, n,
$$

em que $Y_{i}$ é a variável resposta, $\boldsymbol{\beta}=\left(\beta_{1}, \ldots, \beta_{p}\right)^{\top}$ é o vetor de regressores, $\mathbf{x}_{i}^{\top}=\left(x_{i 1}, \ldots, x_{i p}\right)$ é o vetor de variáveis explicativas, tal que $x_{i j}$ é o valor da $j$-ésima variável explicativa para a observação $i \mathrm{e} \stackrel{\text { iid }}{\sim}$ indica independentes e identicamente distribuídos. Pela Definição 1, temos que $Y_{i} \sim \operatorname{SMN}\left(\mathbf{x}_{i}^{\top} \boldsymbol{\beta}, \sigma^{2}, \boldsymbol{\nu}\right)$, para $i=1, \ldots, n$.

Estamos interessados no caso em que as observações com censura à esquerda podem 
ocorrer. Isto é, as observações são da forma

$$
Y_{\mathrm{obs}_{i}}=\left\{\begin{array}{ccc}
\kappa_{i} & \text { if } \quad Y_{i} \leq \kappa_{i} \\
Y_{i} & \text { if } \quad Y_{i}>\kappa_{i}
\end{array}\right.
$$

para $i=1, \ldots, n$. Por conveniência, escolhemos trabalhar com o caso de censura à esquerda, mas os resultados são facilmente estendíveis para outros tipos de censura. Se considerarmos $\kappa_{i}=0$ e assumirmos que $\epsilon_{i} \sim \mathrm{N}\left(0, \sigma^{2}\right)$, isto é, $U_{i}=1$ na Definição $1, i=1, \ldots, n$, obtemos o modelo Tobit censurado estudado por Barros et al. (2010). Além disso, se $U_{i} \sim$ $\operatorname{Gamma}(\nu / 2, \nu / 2)$, obtemos o modelo de regressão censurado t de Student desenvolvido por Arellano-Valle et al. (2012) e Massuia et al. (2012).

É importante definir algumas notações que serão utilizadas ao longo das próximas seções; assim: Seja a variável aleatória $Y_{i} \sim \operatorname{SMN}\left(\mathbf{x}_{i}^{\top} \boldsymbol{\beta}, \sigma^{2}, \boldsymbol{\nu}\right)$ e os parâmetros de interesse $\boldsymbol{\theta}=$ $\left(\beta^{\top}, \sigma^{2}, \boldsymbol{\nu}\right)^{\top}$, então a distância de Mahalanobish é denotada por $d\left(y_{i}, \boldsymbol{\theta}\right)=\left(y_{i}-\mathbf{x}_{i}^{\top} \boldsymbol{\beta}\right)^{2} / \sigma^{2}$, $\widehat{d}\left(y_{i}, \boldsymbol{\theta}\right)=d\left(y_{i}, \widehat{\boldsymbol{\theta}}\right)$ e $\widehat{d}_{k}\left(y_{i}\right)=\widehat{d}\left(y_{i}, \boldsymbol{\theta}^{(k)}\right)=d\left(y_{i}, \widehat{\boldsymbol{\theta}}^{(k)}\right)$.

\subsection{Função de log-verossimilhança}

Seja $\boldsymbol{\theta}=\left(\beta^{\top}, \sigma^{2}, \boldsymbol{\nu}\right)^{\top}$ o vetor dos parâmetros do modelo SMN-CR e $\mathbf{y}_{\text {obs }}$ uma amostra observada. Supondo que há $m$ possíveis valores censurados da variável de interesse, podemos particionar a amostra observada $\mathbf{y}_{\text {obs }}$ em duas subamostras de $m$ valores censurados e $n-m$ valores não censurados, assim $\mathbf{y}_{\mathrm{obs}}=\left\{\kappa_{1}, \ldots, \kappa_{m}, y_{m+1}, \ldots, y_{n}\right\}$.

Então a função de log-verossimilhança, dada a amostra observada $\mathbf{y}_{\text {obs }}$, é dada por

$$
\ell\left(\boldsymbol{\theta} \mid \mathbf{y}_{\text {obs }}\right)=\sum_{i=1}^{m} \log \left[F_{S M N}\left(\frac{\kappa_{i}-\mathbf{x}_{i}^{\top} \boldsymbol{\beta}}{\sigma}\right)\right]+\sum_{i=m+1}^{n} \log \left[f_{S M N}\left(y_{i} \mid \mathbf{x}_{i}^{\top} \boldsymbol{\beta}, \sigma^{2}, \boldsymbol{\nu}\right)\right] .
$$

Para estimar os parâmetros do modelo SMN-CR, uma opção é maximizar a função de log-verossimilhança diretamente, um procedimento que pode ser bastante complicado. Alternativamente, podem ser utilizados o algoritmo EM (Expectation-Maximmization), que é uma adaptação da proposição original de Dempster et al. (1977), ou algumas extensões do algoritmo EM, tais como o ECM (Meng \& Rubin, 1993) e o algoritmo ECME (Liu \& Rubin, 1994). Neste trabalho usaremos o algoritmo ECME, uma ferramenta generalizada confiável para obter estimativas de máxima verossimilhança.

\subsection{Estimação dos parâmetros via algoritmo EM}

Com o objetivo de estimar os parâmetros dos modelos SMN-CR, via algoritmo tipo EM, usaremos a representação estocástica do modelo em termos de distribuições mais tratáveis, que em geral dependem de quantidades não observáveis, denotadas por "dados perdidos". Assim, pela Definição 1, temos a seguinte representação hierárquica:

$$
\begin{aligned}
Y_{i} \mid U_{i}=u_{i} & \sim \mathrm{N}\left(\mathbf{x}_{i}^{\top} \boldsymbol{\beta}, u_{i}^{-1} \sigma^{2}\right) ; \\
U_{i} & \sim H(\cdot \mid \boldsymbol{\nu}) .
\end{aligned}
$$


Se a observação $i$ for censurada, podemos considerar $y_{i}$ como a realização de uma variável latente não observavel $Y_{i} \sim \operatorname{SMN}\left(\mathbf{x}_{i}^{\top} \boldsymbol{\beta}, \sigma^{2}, \boldsymbol{\nu}\right), i=1, \ldots, m$. O procedimento chave para o desenvolvimento do algoritmo tipo EM para o modelo em questão é considerar os dados completos $\mathbf{z}=\left\{\mathbf{y}_{\text {obs }}, y_{1}, \ldots, y_{m}, u_{1}, \ldots, u_{n}\right\}$, isto é, abordamos o problema como se os "dados perdidos" $\mathbf{y}_{L}=\left\{y_{1}, \ldots, y_{m}\right\}$ e $\mathbf{u}=\left\{u_{1}, \ldots, u_{n}\right\}$ fossem de fato observados. Assim, considerando as representações (2.4) e (2.5), a função de log-verossimilhança completa para $\boldsymbol{\theta}$, associada com $\mathbf{z}$, é dada por:

$\ell_{c}(\boldsymbol{\theta} \mid \mathbf{z})=-\frac{n}{2} \log (2 \pi)-\frac{n}{2} \log \left(\sigma^{2}\right)+\frac{1}{2} \sum_{i=1}^{n} \log \left(u_{i}\right)-\frac{1}{2 \sigma^{2}} \sum_{i=1}^{n} u_{i}\left(y_{i}-\mathbf{x}_{i}^{\top} \boldsymbol{\beta}\right)^{2}+\sum_{i=1}^{n} \log \left(h\left(u_{i} \mid \boldsymbol{\nu}\right)\right)$,

em que $h(\cdot \mid \boldsymbol{\nu})$ representa a função de densidade da variável aleatória $U$.

No Passo E do algoritmo, devemos obter a função $Q$, definida por

$$
Q\left(\boldsymbol{\theta} \mid \boldsymbol{\theta}^{(k)}\right)=\mathrm{E}_{\boldsymbol{\theta}^{(k)}}\left[\ell_{c}(\boldsymbol{\theta} \mid \mathbf{Z}) \mid \mathbf{y}_{\mathrm{obs}}\right],
$$

em que $\boldsymbol{\theta}^{(k)}$ é uma atualização da estimativa gerada pelo algoritmo no passo $k$ e $\mathrm{E}_{\boldsymbol{\theta}^{(k)}}$ é a esperança calculada supondo que o vetor $\boldsymbol{\theta}^{(k)}$ contém os verdadeiros valores dos parâmetros $\boldsymbol{\theta}$. Observe que a expressão $Q$ é completamente determinada pelo conhecimento das seguintes esperanças

$$
\mathcal{E}_{s i}\left(\boldsymbol{\theta}^{(k)}\right)=\mathrm{E}_{\boldsymbol{\theta}^{(k)}}\left[U_{i} Y_{i}^{s} \mid y_{\mathrm{obs}_{i}}\right], \quad s=0,1,2,
$$

assim como

$$
\mathrm{E}_{\boldsymbol{\theta}^{(k)}}\left[\log \left(U_{i}\right) \mid y_{\mathrm{obs}_{i}}\right] \text { e } \mathrm{E}_{\boldsymbol{\theta}^{(k)}}\left[\log \left(h\left(U_{i} \mid \boldsymbol{\nu}\right)\right) \mid y_{\mathrm{obs}_{i}}\right] .
$$

Contudo, eliminando as constantes que não dependem de $\boldsymbol{\theta}$, a função $Q$ pode ser escrita de forma reduzida por

$$
\begin{aligned}
Q\left(\boldsymbol{\theta} \mid \boldsymbol{\theta}^{(k)}\right)= & -\frac{n}{2} \log \left(\sigma^{2}\right)-\frac{1}{2 \sigma^{2}} \sum_{i=1}^{n}\left[\mathcal{E}_{2 i}\left(\boldsymbol{\theta}^{(k)}\right)-2 \mathcal{E}_{1 i}\left(\boldsymbol{\theta}^{(k)}\right) \mathbf{x}_{i}^{\top} \boldsymbol{\beta}+\mathcal{E}_{0 i}\left(\boldsymbol{\theta}^{(k)}\right)\left(\mathbf{x}_{i}^{\top} \boldsymbol{\beta}\right)^{2}\right] \\
& +\frac{1}{2} \sum_{i=1}^{n} \mathrm{E}_{\boldsymbol{\theta}^{(k)}}\left[\log \left(U_{i}\right) \mid y_{\mathrm{obs}_{i}}\right]+\sum_{i=1}^{n} \mathrm{E}_{\boldsymbol{\theta}^{(k)}}\left[\log \left(h\left(U_{i} \mid \boldsymbol{\nu}\right)\right) \mid y_{\mathrm{obs}_{i}}\right]
\end{aligned}
$$

Em cada passo, a esperança condicional $\mathcal{E}_{s i}\left(\boldsymbol{\theta}^{(k)}\right)$ pode ser facilmente obtida utilizando os resultados da Proposição 1. Assim, consideraremos os dois casos possíveis para uma determinada observação:

Para a i-ésima observação não censurada, temos que $Y_{\mathrm{obs}_{i}}=Y_{i} \sim \operatorname{SMN}\left(\mathbf{x}_{i}^{\top} \boldsymbol{\beta}, \sigma^{2}, \boldsymbol{\nu}\right)$ e, portanto,

$$
\mathcal{E}_{s i}\left(\boldsymbol{\theta}^{(k)}\right)=y_{i}^{s} \mathrm{E}_{\boldsymbol{\theta}^{(k)}}\left[U_{i} \mid y_{i}\right],
$$

em que $\mathrm{E}_{\boldsymbol{\theta}^{(k)}}\left[U_{i} \mid y_{i}\right]$ pode ser obtida utilizando os resultados de Osorio et al. (2007). Assim, por exemplo,

- Se $Y_{i} \sim \operatorname{PVII}\left(\mathbf{x}_{i}^{\top} \boldsymbol{\beta}, \sigma^{2}, \nu, \delta\right)$, então $\mathrm{E}_{\boldsymbol{\theta}^{(k)}}\left[U_{i} \mid y_{i}\right]=\frac{\nu+1}{\delta+\widehat{d}_{k}\left(y_{i}\right)}$ 
- Se $Y_{i} \sim S L\left(\mathbf{x}_{i}^{\top} \boldsymbol{\beta}, \sigma^{2}, \nu\right)$, então $\mathrm{E}_{\boldsymbol{\theta}^{(k)}}\left[U_{i} \mid y_{i}\right]=\frac{\Gamma\left(\nu+1.5, \widehat{d}_{k}\left(y_{i}\right) / 2\right)}{\Gamma\left(\nu+0.5, \widehat{d}_{k}\left(y_{i}\right) / 2\right)}$

- Se $Y_{i} \sim C N\left(\mathbf{x}_{i}^{\top} \boldsymbol{\beta}, \sigma^{2}, \nu, \gamma\right)$, então $\mathrm{E}_{\boldsymbol{\theta}^{(k)}}\left[U_{i} \mid y_{i}\right]=\frac{1-\nu+\nu \gamma^{1.5} e^{0.5(1-\gamma) \widehat{d}_{k}\left(y_{i}\right)}}{1-\nu+\nu \gamma^{0.5} e^{0.5(1-\gamma) \widehat{d}_{k}\left(y_{i}\right)}}$.

Para a i-ésima observação censurada, temos que $Y_{i} \leq \kappa_{i}$, de modo que

$$
\mathcal{E}_{s i}\left(\boldsymbol{\theta}^{(k)}\right)=\mathrm{E}_{\boldsymbol{\theta}^{(k)}}\left[U_{i} Y_{i}^{s} \mid Y_{i} \leq \kappa_{i}\right]
$$

a qual pode ser obtida para as diferentes distribuições utilizando os resultados obtidos na Proposição 1, conjuntamente com os resultados obtidos através das equações (1.6) e (1.7), com $r=1$.

Quando o passo M contém expressões matemáticas analiticamente intratáveis, estas podem ser substituídas por uma sequência de passos de maximização condicional (CM). Este procedimento implementado por Meng \& Rubin (1993) é conhecido como Algoritmo ECM. Uma extensão do algoritmo EM e ECM é o Algoritmo ECME (Liu \& Rubin, 1994), que é obtido maximizando a correspondente função de verossimilhança marginal restrita ao conhecimento dos parâmetros no paso anterior.

\section{Passo E:}

Dado $\boldsymbol{\theta}=\boldsymbol{\theta}^{(k)}$, para $i=1, \ldots, n$, temos que

- Se a $i$-ésima observação for não censurada, então, para $s=0,1,2$, calculamos $\mathcal{E}_{s i}\left(\boldsymbol{\theta}^{(k)}\right)$, dado em (2.8);

- Se a $i$-ésima observação for censurada, então, para $s=0,1,2$, obtemos $\mathcal{E}_{s i}\left(\boldsymbol{\theta}^{(k)}\right)$, dado em (2.9).

\section{Passo CM:}

Atualizamos $\boldsymbol{\theta}^{(k)}$ pela maximização $Q\left(\boldsymbol{\theta} \mid \boldsymbol{\theta}^{(k)}\right)$ sobre $\boldsymbol{\theta}$, o que leva as seguintes expressões:

$$
\begin{aligned}
\boldsymbol{\beta}^{(k+1)} & =\left(\sum_{i=1}^{n} \mathcal{E}_{0 i}\left(\boldsymbol{\theta}^{(k)}\right) \mathbf{x}_{i} \mathbf{x}_{i}^{\top}\right)^{-1} \sum_{i=1}^{n} \mathbf{x}_{i} \mathcal{E}_{1 i}\left(\boldsymbol{\theta}^{(k)}\right) \\
\sigma^{2(k+1)} & =\frac{1}{n} \sum_{i=1}^{n}\left[\mathcal{E}_{2 i}\left(\boldsymbol{\theta}^{(k)}\right)-2 \mathcal{E}_{1 i}\left(\boldsymbol{\theta}^{(k)}\right) \mathbf{x}_{i}^{\top} \boldsymbol{\beta}^{(k+1)}\right. \\
& \left.+\mathcal{E}_{0 i}\left(\boldsymbol{\theta}^{(k)}\right)\left(\mathbf{x}_{i}^{\top} \boldsymbol{\beta}^{(k+1)}\right)^{2}\right] .
\end{aligned}
$$

\section{Passo CML:}

Atualizamos $\nu^{(k)}$ pela maximização da função de verossimilhança marginal, o que leva a seguinte expressão

$$
\begin{aligned}
\boldsymbol{\nu}^{(k+1)}= & \operatorname{argmax}_{\nu}\left\{\sum_{i=1}^{m} \log \left[F_{S M N}\left(\frac{\kappa_{i}-\mathbf{x}_{i}^{\top} \boldsymbol{\beta}^{(k+1)}}{\sigma^{(k+1)}}\right)\right]\right. \\
& \left.+\sum_{i=m+1}^{n} \log \left[f_{S M N}\left(y_{i} \mid \mathbf{x}_{i}^{\top} \boldsymbol{\beta}^{(k+1)}, \sigma^{2^{(k+1)}}, \boldsymbol{\nu}\right)\right]\right\} .
\end{aligned}
$$


Este processo é iterado até que algum critério de convergência seja satisfeito. Por exemplo, quando a distância envolvendo duas avaliações sucessivas da log-verossimilhança $\ell\left(\boldsymbol{\theta} \mid \mathbf{y}_{\text {obs }}\right)$, dada por

$$
\left\|\ell\left(\boldsymbol{\theta}^{(k+1)} \mid \mathbf{y}_{o b s}\right)-\ell\left(\boldsymbol{\theta}^{(k)} \mid \mathbf{y}_{o b s}\right)\right\| \quad \text { ou } \quad\left\|\ell\left(\boldsymbol{\theta}^{(k+1)} \mid \mathbf{y}_{o b s}\right) / \ell\left(\boldsymbol{\theta}^{(k)} \mid \mathbf{y}_{o b s}\right)-1\right\|,
$$

for suficientemente pequena. Adotamos esta estratégia para atualizar a estimativa de $\boldsymbol{\nu}$ pela maximização direta da log-verossimilhança marginal com o intuito de contornar o cálculo de $\mathrm{E}_{\boldsymbol{\theta}^{(k)}}\left[\log \left(U_{i}\right) \mid y_{\mathrm{obs}_{i}}\right]$ e $\mathrm{E}_{\boldsymbol{\theta}^{(k)}}\left[\log \left(h\left(U_{i} \mid \boldsymbol{\nu}\right)\right) \mid y_{\mathrm{obs}_{i}}\right]$.

\subsection{Erro padrão aproximado para os efeitos fixos}

Neste texto, iremos denotar o estimador de máxima verossimilhança (MV) de $\boldsymbol{\theta}$ por $\widehat{\boldsymbol{\theta}}=\left(\widehat{\boldsymbol{\beta}}, \widehat{\sigma^{2}}, \widehat{\nu}\right)^{\top}$. Com o intuito de avaliar sua variabilidade, a matriz de variância-covariância estimada, para as observações censuradas, é ajustada utilizando a fórmula de Louis (Louis, 1982). Veja também Vaida et al. (2007, Sec. 2), Vaida \& Liu (2009, Sec.2) e Matos et al. (2013, Sec.3) para maiores detalhes.

A log-verossimilhança obtida a partir da $i$-ésima observação completa $\mathbf{z}_{i}=\left(y_{\mathrm{obs}_{i}}, y_{i}, u_{i}\right)^{\top}$ será denotada por $\ell_{c}\left(\boldsymbol{\theta} \mid \mathbf{z}_{i}\right)$, enquanto $\operatorname{Var}_{\boldsymbol{\theta}}$ denota a matriz de variância-covariância calculada utilizando $\boldsymbol{\theta}$ como os valores reais dos parâmetros.

A estimação da $\operatorname{Var}_{\boldsymbol{\theta}}[\widehat{\boldsymbol{\beta}}]$ é dada pela inversa da matriz

$$
-\sum_{i=1}^{n}\left\{\mathrm{E}_{\boldsymbol{\theta}}\left[\frac{\partial^{2} \ell\left(\boldsymbol{\theta} \mid y_{o b s_{i}}\right)}{\partial \boldsymbol{\beta} \partial \boldsymbol{\beta}^{T}}\right]-\operatorname{Var}_{\boldsymbol{\theta}}\left[\frac{\partial \ell_{c}\left(\boldsymbol{\theta} \mid \mathbf{Z}_{i}\right)}{\partial \boldsymbol{\beta}} \mid y_{o b s_{i}}\right]\right\}
$$

avaliada em $\widehat{\boldsymbol{\theta}}$.

Como as distribuições SMN também são distribuições elípticas, com

$$
g(z)=\int_{0}^{\infty} \sqrt{u} \exp \{-(u / 2) z\} d H(u \mid \boldsymbol{\nu})
$$

é possível provar que a Matriz de Informação sob o modelo R-SMN (2.1), isto é, o modelo não censurado), é bloco diagonal em que o bloco correspondente a $\boldsymbol{\beta}$ é dado por

$$
-\sum_{i=1}^{n} \mathrm{E}_{\boldsymbol{\theta}}\left[\frac{\partial^{2} \ell\left(\boldsymbol{\theta} \mid y_{o b s_{i}}\right)}{\partial \boldsymbol{\beta} \partial \boldsymbol{\beta}^{T}}\right]=\frac{4}{\sigma^{2}} \sum_{i=1}^{n}\left(\mathbf{x}_{i} \mathbf{x}_{i}^{\top}\right) d_{g i},
$$

em que $d_{g i}=\mathrm{E}\left[Z_{i}^{2} W_{g}^{2}\left(Z_{i}^{2}\right)\right], Z_{i} \sim \operatorname{SMN}(0,1, \boldsymbol{\nu})$ e $W_{g}(z)=g^{\prime}(z) / g(z)$, em que $g^{\prime}(z)$ é a primeira derivada de $g(\cdot)$ em relação a $z$. Para algumas distribuições da família SMN, a expressão $q(z)=-2 W_{g}(z)$ tem formas analiticamente tratáveis, como por exemplo:

- Normal: $q(z)=1$;

- $\mathrm{t}$ de Student: $q(z)=(\nu+1) /(\nu+z)$;

- Slash: $q(z)=\frac{\Gamma(\nu+1.5, z / 2)}{\Gamma(\nu+0.5, z / 2)}$; 
- Normal contaminada:

$$
q(z)=\frac{1-\nu+\nu \gamma^{\frac{3}{2}} \exp \{(1 / 2)(1-\gamma) z\}}{1-\nu+\nu \gamma^{\frac{1}{2}} \exp \{(1 / 2)(1-\gamma) z\}}
$$

Para maiores detalhes, veja Osorio et al. (2007, Sec 2).

Para o caso da distribuição t de Student, Lange et al. (1989) mostraram que $d_{g i}=$ $(\nu+1) / 4(\nu+3)$. Para as distribuições Slash e normal contaminada (e outras distribuições elípticas) o cálculo de $d_{g i}$ involve integrais complexas, as quais podem ser resolvidas utilizando técnicas Monte Carlo.

A Matriz de Informação ajustada, para as observações censuradas, é dada por

$$
\begin{aligned}
\sum_{i=1}^{m} \operatorname{Var}_{\boldsymbol{\theta}}\left[\frac{\partial \ell_{c}\left(\boldsymbol{\theta} \mid \mathbf{Z}_{i}\right)}{\partial \boldsymbol{\beta}} \mid Y_{i} \leq \kappa_{i}\right] & =\sum_{i=1}^{m} \operatorname{Var}_{\boldsymbol{\theta}}\left[\frac{1}{\sigma^{2}} \mathbf{x}_{i}\left(U_{i} Y_{i}-U_{i} \mathbf{x}_{i}^{\top} \boldsymbol{\beta}\right) \mid Y_{i} \leq \kappa_{i}\right] \\
& =\frac{1}{\sigma^{4}} \sum_{i=1}^{m}\left(\mathbf{x}_{i} \mathbf{x}_{i}^{\top}\right)\left[\operatorname{Var}_{\boldsymbol{\theta}}\left\{U_{i} Y_{i}-U_{i} \mathbf{x}_{i}^{\top} \boldsymbol{\beta} \mid Y_{i} \leq \kappa_{i}\right\}\right] \\
& =\frac{1}{\sigma^{4}} \sum_{i=1}^{m}\left(\mathbf{x}_{i} \mathbf{x}_{i}^{\top}\right)\left\{\operatorname{Var}_{\boldsymbol{\theta}}\left[U_{i} Y_{i}^{\top} \mid Y_{i} \leq \kappa_{i}\right]\right. \\
& +\operatorname{Var}_{\boldsymbol{\theta}}\left[U_{i} \mathbf{x}_{i}^{\top} \boldsymbol{\beta} \mid Y_{i} \leq \kappa_{i}\right] \\
& \left.-2 \operatorname{Cov}_{\boldsymbol{\theta}}\left[U_{i} Y_{i}, U_{i} \mathbf{x}_{i}^{\top} \boldsymbol{\beta} \mid Y_{i} \leq \kappa_{i}\right]\right\}
\end{aligned}
$$

em que Cov denota Covariância.

\subsection{Seleção de modelos}

Alguns critérios de seleção de modelos geralmente utilizados são: o Critério de Informação de Akaike (AIC), o Critério de Informação Bayesiano (BIC) e o Critério de Determinação de Eficiência (EDC), veja Bai et al. (1989), que são definidos por

$$
-2 \ell(\widehat{\boldsymbol{\theta}} \mid \mathbf{y})+\gamma c_{n}
$$

em que $\gamma$ é o número de parâmetros livres que são estimados no modelo e o termo de penalização $c_{n}$ é uma sequência (conveniente) de números positivos. Temos que $c_{n}=2$ para o $\mathrm{AIC}, c_{n}=\log (n)$ para o BIC, e para o critério EDC, $c_{n}$ é escolhido de modo a satisfazer as condições $c_{n} / n \rightarrow 0$ e $c_{n} /(\log n) \rightarrow 0$, quando $n \rightarrow \infty$. Aqui, utilizamos $c_{n}=0.2 \sqrt{n}$, como considerado em Bai et al. (1989).

\subsection{Análise de resíduos}

A análise de resíduos tem como finalidade identificar observações atípicas, assim como verificar se as hipóteses do modelo considerado são satisfeitas.

Neste trabalho, avaliou-se os resíduos martingais transformados $r_{M T_{i}}$, propostos por Barros et al. (2010), que são definidos como

$$
r_{M T_{i}}=\operatorname{sign}\left(r_{M_{i}}\right) \sqrt{-2\left[r_{M_{i}}+\delta_{i} \log \left(\delta_{i}-r_{M_{i}}\right)\right]}, \quad i=1, \ldots, n,
$$


em que $r_{M_{i}}=\delta_{i}+\log S\left(y_{i}, \widehat{\boldsymbol{\theta}}\right)$ é o resíduo martingal proposto por Ortega et al. (2003) e trabalhado por Therneau et al. (1990), $\delta_{i}=0,1$ indica se a $i$-ésima observação é censurado ou não, respectivamente, $\operatorname{sign}\left(r_{M_{i}}\right)$ denota o signal de $r_{M_{i}}$ e $S\left(y_{i}, \widehat{\boldsymbol{\theta}}\right)=P_{\widehat{\boldsymbol{\theta}}}\left(Y_{i}>y_{i}\right)$ representa a função de sobrevivência avaliada em $y_{i}$, em que $\widehat{\boldsymbol{\theta}}$ representa as estimações de MV para $\boldsymbol{\theta}$.

Usamos este tipo de resíduos padronizados devido a que são simétricamente distribuidos ao redor de zero, o que facilitará a construção dos envelopes simulados com pouco esforço computacional e será util para detectar uma especificação incorreta do modelo, assim como, presença de observações atípicas.

\subsection{Estudos de Simulação}

Com o intuito de avaliar o desempenho das estimativas obtidas nos modelos SMN-CR utilizando as metodologias desenvolvidas nas Seções 2.4 e 2.5, apresentamos três estudos de simulação. A primeira parte de este estudo de mostra a necessidade de modelo com caudas mais pesadas que a da distribuição normal para lidar com a presença de valores outliers nos dados. O objetivo da segunda parte é mostrar que as estimativa de MV obtidas através do nosso algoritmo tipo EM têm boas propriedades assintóticas. Finalmente, a terceira parte de esta simulação visa avaliar o desempenho da metodologia utilizada na Seção 2.5 para estimar o erros padrões assintóticos dos parâmetros de efeitos fixos estimados.

Para os três estudos de simulação foi considerado inicialmente o modelo censurado SMNCR, definido em (2.1) e (2.2), com $\mathbf{x}_{i}^{\top}=\left(1, x_{i}\right), \varepsilon_{i} \sim \operatorname{SMN}\left(0, \sigma^{2}\right), i=1, \ldots, n$ e um percentagem de nível de censura fixado $p$. Os procedimentos computacionais foram implementados utilizando o software R (R Core Team, 2013).

\subsubsection{Simulação 1: Robustez das estimativas de MV}

O objetivo deste estudo de simulação é comparar o desempenho das estimativas de MV para os modelos de regressão censurados SMN-CR na presença de valores outliers sobre a variável resposta.

Considerando inicialmente o modelo linear censurado N-CR, geramos 1000 amostras aleatórias de tamanho $n=100, \operatorname{com} \boldsymbol{\beta}^{\top}=\left(\beta_{1}, \beta_{2}\right)=(1,4), \sigma^{2}=2$ e um nivel de censura fixado de $p=8 \%$ (isto é, $8 \%$ das observações foram censuradas). Os valores $x_{i}, i=1, \ldots, n$, foram geradas independentemente de uma distribuição $U(2,20)$ e foram fixados para todas as amostras.

Para avaliar como as estimativas de MV são influenciados pela presença de outliers, substituimos a observação $y_{50}$ por $y_{50}(\vartheta)=y_{50}-\vartheta, \operatorname{com} \vartheta=1,2, \ldots, 10$. Assim, seja $\widehat{\beta}_{i}(\vartheta)$ a estimativa de MV de $\beta_{i}$ depois da contaminação $\vartheta$, para $i=1,2$, estamos particularmente interessados no cálculo da mudança relativa, definida por

$$
R C\left(\beta_{i}\right)=\left|\left(\widehat{\beta}_{i}(\vartheta)-\widehat{\beta}_{i}\right) / \widehat{\beta}_{i}\right| .
$$


Analogamente, podemos definir a mudança relativa para o parâmetro $\sigma^{2}$.

Para cada réplica obtemos os parâmetros estimados com e sem outliers, sob os seguintes modelos: N-CR, T-CR com diferentes valores fixados $\nu \in\{3,6,8,10,12\}$, SL-CR com valores fixos de $\nu \in\{3,4\}$ e CN-CR com $\boldsymbol{\nu}^{\top}=(\xi, \gamma)=(0.3,0.3)$ fixado. A Figura 2.1 mostra os valores médios da mudança relativa nas estimativas de MV nos modelos e observamos que no caso do modelo $\mathrm{N}-\mathrm{CR}$ a influência se incrementa significativamente a medida que $\vartheta$ aumenta. No entanto, para os modelos SMN-CR com caudas mais pesadas como T-CR e SL-CR como diferentes valores de $\nu$, estas mudanças relativa não se incrementam de forma significativa, o qual indica que estes modelos são muito mais robustos que o modelo N$\mathrm{CR}$ na presença de valores outliers nas observações. No caso do modelo $\mathrm{CN}-\mathrm{CR}$ podemos observar que só no parâmetro $\sigma^{2}$, a mudança reativa média se incrementa significativamente a medida que $\vartheta$ aumenta.
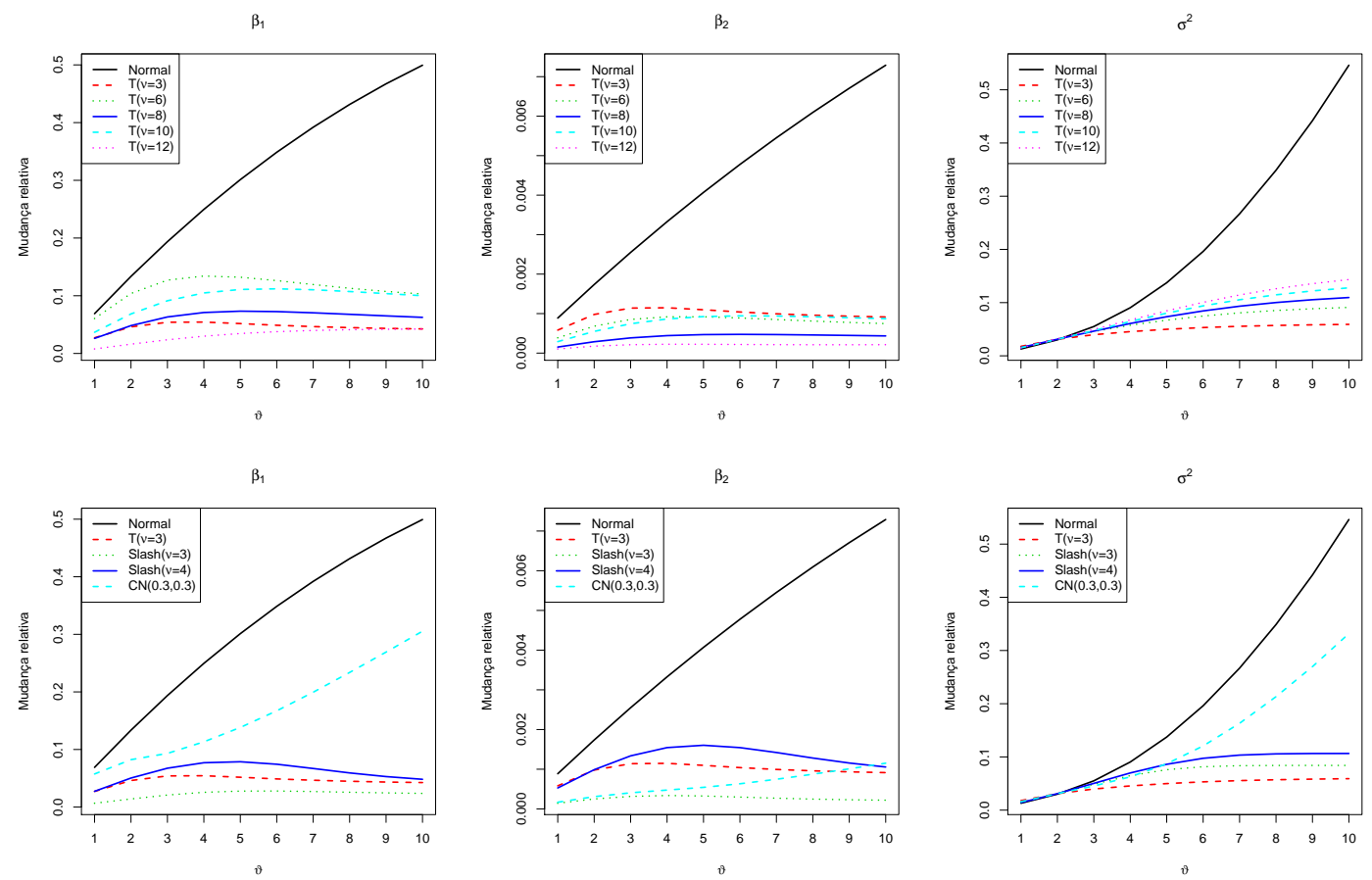

Figura 2.1: Mudança relativa média nas estimativas de MV para diferentes valores de $\vartheta$.

\subsubsection{Simulação 2: Propriedades Assintóticas}

Este estudo de simulação foi desenvolvido com o intuito de avaliar o desempenho das estimações dos parâmetros obtidos, utilizando nosso algoritmo tipo EM para diferentes tamanhos de amostra. Para isto, consideramos o modelo linear SMN-CR com um nível de censura de $p=10 \%$. Os tamanhos da amostra são fixados como $n=50,100,150,200$, 300, 400, 500, 700 e 800. Os valores considerados para os parâmetros da regressão foram $\boldsymbol{\beta}^{\top}=\left(\beta_{1}, \beta_{2}\right)=(1.5,4)$ e $\sigma^{2}=0.5$. Como definido em Labra et al. (2012), utilizamos para a variável $x_{i}$ uma sequência de valores entre 0.1 e 20 , e esses valores foram fixados para 
todo o experimento.

Considerando os diferentes tamanhos da amostra, os valores dos parâmetros e o nível de censura, foram geradas 1000 amostras do modelo SMN-CR sob quatro diferentes distribuições: N-CR, T-CR $(\nu=3)$, SL-CR $(\nu=4)$ e CN-CR $\left(\boldsymbol{\nu}^{\top}=(0.5,0.5)\right)$. Para cada amostra gerada, obtivemos as estimações dos parâmetros, logo calculamos os valores médios do Viés e o Erro Quadrático Médio (EQM) tal que, para $\widehat{\boldsymbol{\theta}}_{i}$, estimador de $\boldsymbol{\theta}_{i}$, são dados respectivamente por

$$
\text { Viés }\left(\widehat{\boldsymbol{\theta}}_{i}\right)=\frac{1}{1000} \sum_{j=1}^{1000}\left(\widehat{\boldsymbol{\theta}}_{i}^{(j)}-\boldsymbol{\theta}_{i}\right) \quad \text { e } \operatorname{EQM}\left(\widehat{\boldsymbol{\theta}}_{i}\right)=\frac{1}{1000} \sum_{j=1}^{1000}\left(\widehat{\boldsymbol{\theta}}_{i}^{(j)}-\boldsymbol{\theta}_{i}\right)^{2} \text {, }
$$

em que $\widehat{\boldsymbol{\theta}}_{i}^{(j)}$ é a estimação de $\boldsymbol{\theta}_{i}$ para a $j$-ésima amostra e $\boldsymbol{\theta}=\left(\boldsymbol{\beta}, \sigma^{2}, \boldsymbol{\nu}\right)$.

Os resultados são apresentados através da Figura 2.2, em que podemos observar que o Viés e o EQM médios convergem ambos para zero à medida que $n$ aumenta, indicando que as estimativas de MV obtidas através do algoritmo tipo EM proposto proporciona boas propriedades assintóticas.
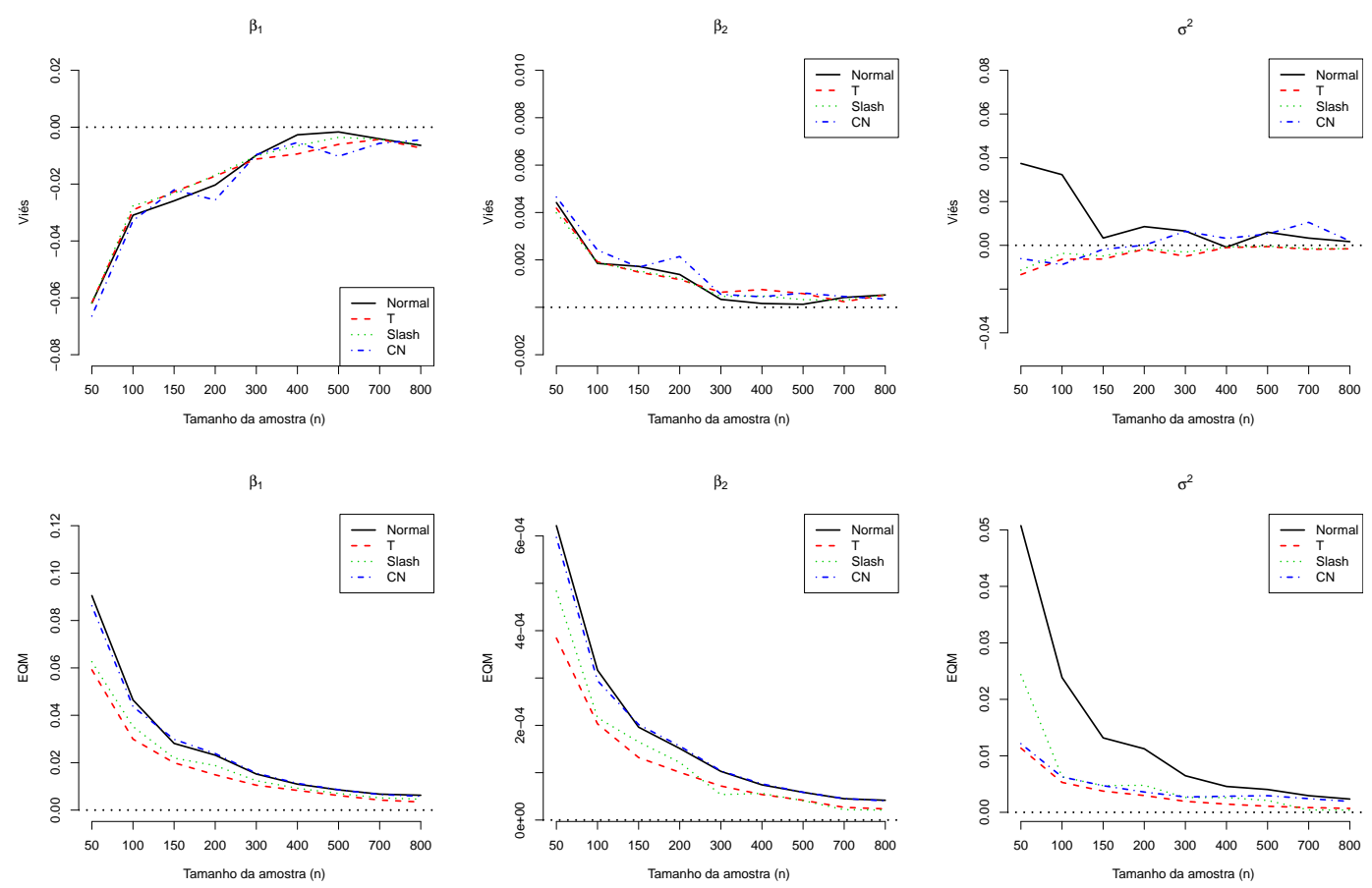

Figura 2.2: Viés médio (primeira linha) e EQM (segunda linha) dos parâmetros estimados nos modelos SMN-CR, para $p=10 \%$.

\subsubsection{Simulação 3: Performance dos Erros Padrões}

Neste estudo de simulação, mostraremos que o método sugerido na Seção 2.5 para aproximar o erro padrão (EP) das estimativas de MV dos parâmetros de regressão tem boas propriedades assintóticas. Fixamos os diferentes modelos lineares SMN-CR (N-CR, T-CR 
Tabela 2.1: Erro padrão amostral de $\widehat{\beta}_{i}$ (MC EP), MC IM EP e COV MC.

\begin{tabular}{cccccccc}
\hline \multirow{2}{*}{$\begin{array}{c}\text { Nível de } \\
\text { censura }\end{array}$} & \multirow{2}{*}{ Medida } & \multicolumn{2}{c}{ N-CR } & \multicolumn{2}{c}{ T-CR } & \multicolumn{2}{c}{ SL-CR } \\
\hline \multirow{2}{*}{$5 \%$} & & $\widehat{\beta}_{1}$ & $\widehat{\beta}_{2}$ & $\widehat{\beta}_{1}$ & $\widehat{\beta}_{2}$ & $\widehat{\beta}_{1}$ & $\widehat{\beta}_{2}$ \\
& MC EP & 0,3445 & 0,1758 & 0,4044 & 0,2050 & 0,4041 & 0,2063 \\
& MC IM EP & 0,3650 & 0,1892 & 0,4354 & 0,2253 & 0,4279 & 0,2246 \\
& COV MC & $96,1 \%$ & $96,6 \%$ & $96,6 \%$ & $97,6 \%$ & $95,4 \%$ & $95,6 \%$ \\
\hline \multirow{2}{*}{$10 \%$} & MC EP & 0,3711 & 0,1884 & 0,4258 & 0,2116 & 0,4047 & 0,2034 \\
& MC IM EP & 0,3693 & 0,1899 & 0,4553 & 0,2358 & 0,4441 & 0,2325 \\
& COV MC & $93,0 \%$ & $93,7 \%$ & $96,2 \%$ & $96,7 \%$ & $96,2 \%$ & $97,1 \%$ \\
\hline \multirow{2}{*}{$20 \%$} & MC EP & 0,3505 & 0,1804 & 0,4056 & 0,2053 & 0,4107 & 0,2099 \\
& MC IM EP & 0,3707 & 0,1848 & 0,4577 & 0,2290 & 0,4497 & 0,2252 \\
& COV MC & $95,1 \%$ & $94,9 \%$ & $97,0 \%$ & $96,9 \%$ & $96,7 \%$ & $96,7 \%$ \\
\hline $50 \%$ & MC EP & 0,3856 & 0,2114 & 0,4637 & 0,2589 & 0,4382 & 0,2361 \\
& MC IM EP & 0,3629 & 0,1748 & 0,4415 & 0,2089 & 0,4440 & 0,2121 \\
& COV MC & $93,3 \%$ & $89,8 \%$ & $93,2 \%$ & $86,9 \%$ & $95,3 \%$ & $91,2 \%$ \\
\hline
\end{tabular}

or SL-CR) e o nível de censura $(5 \%, 10 \%, 20 \%$ ou 50\%). Para cada uma das 12 combinações do modelo e nível de censura, geramos 1000 amostras de tamanho $n=100 \mathrm{com}$ $\boldsymbol{\beta}^{\top}=\left(\beta_{1}, \beta_{2}\right)=(2,1)$ e $\sigma^{2}=0,5$. Para cada amostra, obtemos as estimativas de MV de $\beta_{1}$ e $\beta_{2}$ e as estimativas dos seus erros padrões, utilizando a técnica proposta na Seção 2.5, assim como um intervalo de confiança assintótico assumindo normalidade de $95 \%$.

Na Tabela 4.1 se apresenta o erro padrão amostral de $\widehat{\beta}_{i}$, que é definido por,

$$
\mathrm{MC} \mathrm{EP}=\frac{1}{999}\left[\sum_{i=1}^{1000}\left(\widehat{\beta}_{i}\right)^{2}-\frac{1}{1000}\left(\sum_{i=1}^{1000} \widehat{\beta}_{i}\right)^{2}\right],
$$

o valor médio (considerando as 1000 amostras) dos erros padrões obtidos utilizando o método da informação (IM MC EP) e a percentagem de cobertura, denotada por COV MC, a qual é obtida calculando a percentagem de vezes que os intervalos de confianças cobrem o verdadeiro valor do parâmetro. 


\subsection{Pacote SMNCensReg}

\subsubsection{Introdução}

O pacote "SMNCensReg" (Garay et al., 2013) é uma ferramenta desenvolvida com o objetivo de encontrar as estimativas de MV (via algoritmo tipo EM) e os erros padrões dos parâmetros dos modelos censurados SMN-CR, incluindo as distribuições normal (Normal), t de Student (T), Pearson tipo VII (PearsonVII), slash (Slash) e normal contaminada $($ NormalC $)$. Este pacote está disponível no repositório CRAN e pode ser instalado livremente a partir de:

http://cran.ism.ac.jp/web/packages/SMNCensReg/index.html. As funções são continuamente atualizadas, com o intuito de incorporar novas ferramentas que sejam de utilidade para o usuário, de forma que permitam análises estatísticas mais completas, como por exemplo a análise de diagnóstico, entre outras.

\subsubsection{Descrição}

O comando principal que fornece as estimativas de MV é a função "CensReg.SMN", como pode ser visto a seguir:

Códigos em $\mathrm{R}$

CensReg. SMN (cc , x , y , LS=NULL , nu=3, delta=NULL , cens=" 1 ",
type="T", show . envelope="FALSE", error=0.0001, iter. $\max =300$ )

Com os seguintes argumentos:

- $c c \quad$ : vetor de indicadores de censuras. 0 se não for censurado, 1 se for censurado.

- $x \quad$ : vetor de covariáveis.

- y : vetor de respostas, em caso de censura à esquerda/direita ou vetor do limite inferior no caso de censura intervalar.

- $L S \quad$ : vetor do limite superior, se o tipo de censura for intervalar.

- nu : valor inicial da variável escala (vetor ou escalar) da família SMN. Para a distribuição "NormalC", deve ser um vetor de tamanho dois, com valores entre $(0,1)$.

- delta : valor inicial do segundo parâmetro da distribuição "PearsonVII". Se não for especificado, considera-se $\delta=1$.

- cens : tipo de censura. "1" para censura à esquerda, "2" para censura à direita e "3" para censura intervalar. 
- type : distribuições a serem consideradas na estimação ("Normal", "T", "PearsonVII", "Slash" e "NormalC")

- show.envelope : se for TRUE, será construído o gráfico do envelope baseado nos resíduos martingais transformados.

- error : erro máximo para a convergência.

- iter.max : número máximo de iterações considerados para o algoritmo EM.

\subsubsection{Utilização do pacote}

Com o intuito de exemplificar a utilização do pacote SMNCensReg, definiremos a seguinte sequência de passos:

- Passo 1: Suponha que queremos estimar os parâmetros de um modelo linear Normal, com um nível de censura à esquerda de $p=0.08 \mathrm{~N}-\mathrm{CR}$, definido por:

$$
Y_{i}=\beta_{1}+\beta_{2} x_{i}+\varepsilon_{i}, \quad \varepsilon_{i} \stackrel{\mathrm{iid}}{\sim} \mathrm{N}\left(0, \sigma^{2}\right), \quad i=1, \ldots, n,
$$

$\mathrm{e}$

$$
Y_{\mathrm{obs}_{i}}=\left\{\begin{array}{ccc}
\kappa_{i} & \text { if } & Y_{i} \leq \kappa_{i} \\
Y_{i} & \text { if } & Y_{i}>\kappa_{i} .
\end{array}\right.
$$

- Passo 2: Considerando o modelo linear definido no Passo 1, geramos uma amostra aleatória Normal, com censura à esquerda, de tamanho $n=150$. Cada valor de $x_{i}$ foi gerado considerando uma distribuição uniforme no intervalo $(2,20)$, e os valores verdadeiros dos parâmetros considerados para nosso estudo foram: $\beta_{1}=1, \beta_{2}=4$, e $\sigma^{2}=2$. Esta amostra N-CR é gerada no R, utilizando a seguinte sequência de commandos:

Códigos em $\mathrm{R}$

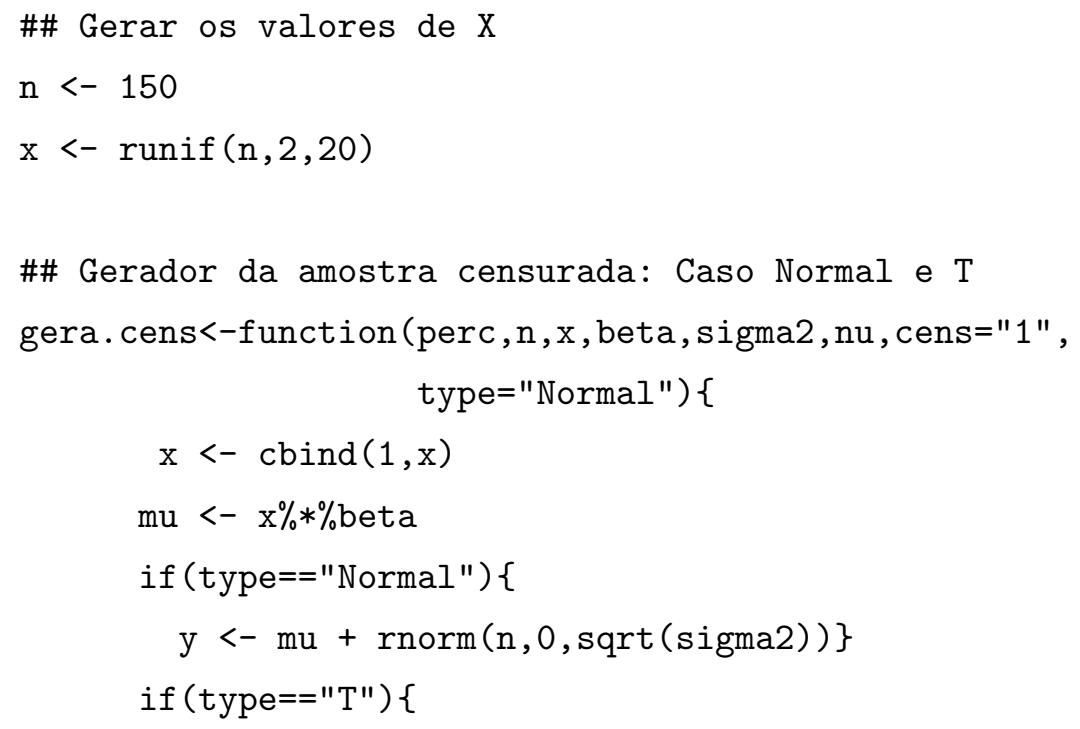




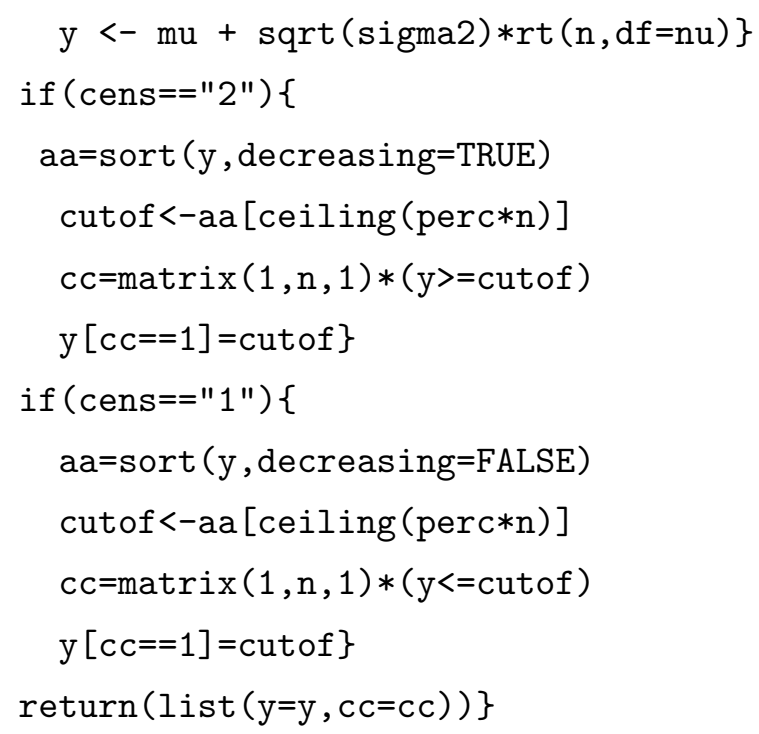

- Passo 3: Uma vez gerada a amostra censurada N-CR, a seguinte sequência de comandos fornece as estimativas dos parâmetros:

Códigos em $\mathrm{R}$

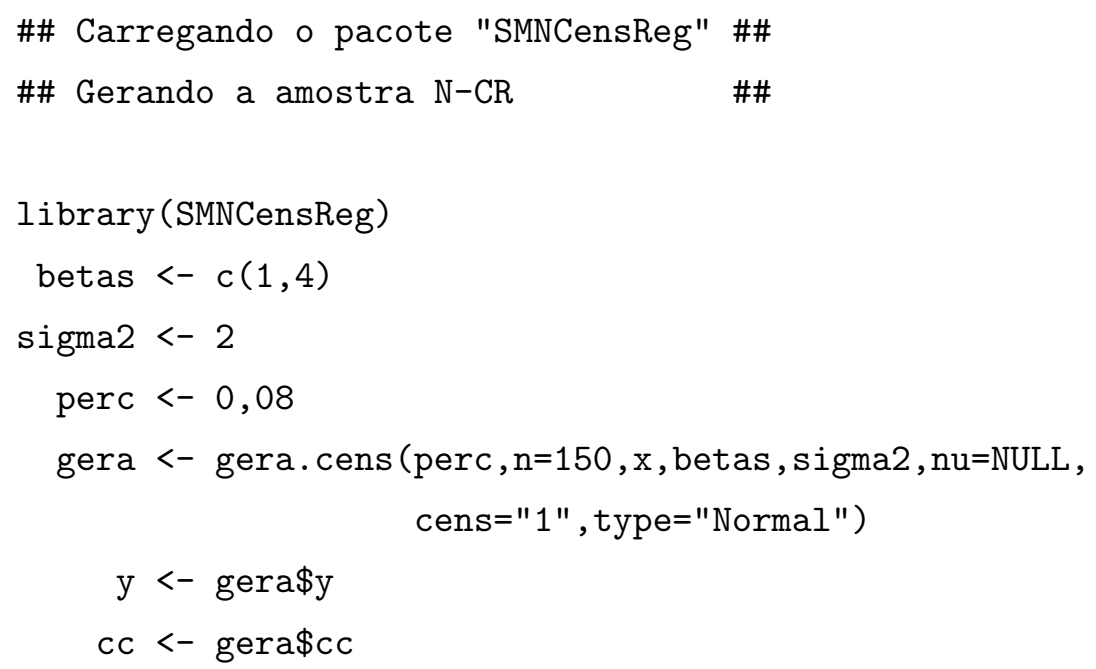

\#\# Encontrando as estimativas de MV

Normal <- CensReg. SMN (cc , x, y, LS=NULL , nu=NULL , delta=NULL, cens $=" 1 "$, type="Normal" , show . envelope="FALSE", error=0.0001, iter $\cdot \max =300$ ) 
- Passo 4: Resultados obtidos com o pacote SMNCensReg:

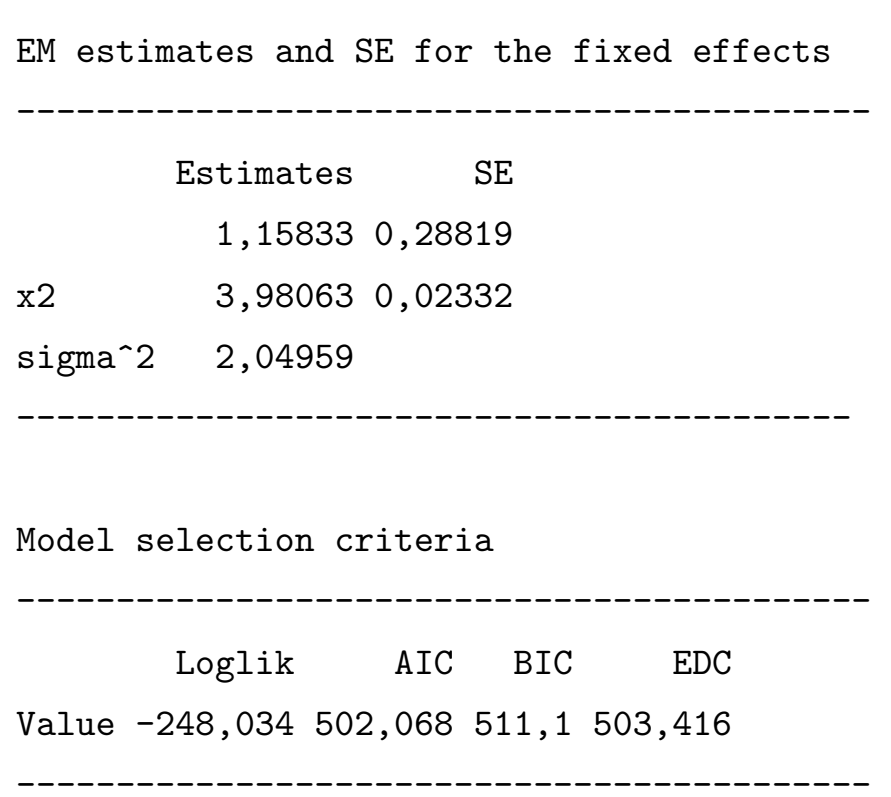

\subsection{Aplicação: Salários}

Nesta seção, utilizaremos os dados referentes a salários analisados por Mroz (1987), em que são avaliados o rendimento médio por hora de 753 mulheres brancas casadas, com idades entre 30 e 60 anos. Este estudo foi desenvolvido durante o ano 1975, e das 753 mulheres consideradas neste estudo, 428 trabalharam em algum momento durante esse ano.

Assim, as variáveis consideradas no estudo para o $i$-ésimo indivíduo foram:

- $y_{i}$ : rendimento médio por hora. Se o valor do rendimento for igual a zero, significa que essas mulheres não trabalharam no ano do estudo. Assim essas observações serão consideradas como observações censuradas em zero. (censura à esquerda)

- $x_{i 2}$ : idade.

- $x_{i 3}$ : anos de escolaridade.

- $x_{i 4}$ : o número de crianças menores de seis anos de idade na casa.

- $x_{i 5}$ : o número de crianças com idade entre seis e dezenove anos de idade na casa.

Cada vetor de variáveis explicativas é dado por $\mathbf{x}_{i}^{\top}=\left(1, x_{2 i}, x_{3 i}, x_{4 i}, x_{5 i}\right)$, para $i=1,2, \ldots, 753$.

Estes dados foram analisados por Arellano-Valle et al. (2012), utilizando o modelo T-CR. Aqui, apresentamos este conjunto de dados com o intuito de ilustrar as técnicas desenvolvidas para a estimação dos parâmetros do modelo censurado (veja Seção 2.4), substituindo a suposição da distribuição T-CR pela classe geral de distribuições SMN. 


\subsubsection{Ajuste do modelo}

Para ajustar o modelo proposto, consideraremos as distribuições normal (N-CR), t de Student (T-CR), slash (SL-CR) e normal contaminada (CN-CR). A Tabela 2.2 mostra as estimativas de MV dos parâmetros para os quatro modelos e seus correspondentes erros padrões (EP). Embora as estimativas dos parâmetros de regressão sejam semelhantes para as distribuições consideradas, os erros padrões obtidos são menores quando consideramos distribuições com caudas mais pesadas do que as do modelo Normal.

A Tabela 2.3 apresenta os valores dos critérios de seleção de modelos: AIC (Akaike, 1974), BIC (Schwarz, 1978) e EDC (Bai et al., 1989). Estes resultados indicam que os três modelos com caudas mais pesadas que a normal (N-CR) apresentam melhores ajustes.

Tabela 2.2: Estimativas de MV e erros padrões (EP) obtidos no ajuste de vários modelos SMN-CR para os Salários.

\begin{tabular}{crrrrrrrr}
\hline & \multicolumn{2}{c}{ N-CR } & \multicolumn{2}{c}{ T-CR } & \multicolumn{2}{c}{ SL-CR } & \multicolumn{2}{c}{ CN-CR } \\
Parameter & Estimate & SE & Estimate & SE & Estimate & SE & Estimate & SE \\
\hline$\beta_{1}$ & $-2,7510$ & 1,7326 & $-1,0470$ & 1,5837 & $-1,4359$ & 1,4983 & $-1,2901$ & 1,4683 \\
$\beta_{2}$ & $-0,1045$ & 0,0274 & $-0,1107$ & 0,0254 & $-0,1072$ & 0,0239 & $-0,1064$ & 0,0234 \\
$\beta_{3}$ & 0,7281 & 0,0825 & 0,6475 & 0,0777 & 0,6545 & 0,0722 & 0,6468 & 0,0708 \\
$\beta_{4}$ & $-3,0263$ & 0,4345 & $-3,1637$ & 0,4800 & $-3,0518$ & 0,3997 & $-3,0649$ & 0,3911 \\
$\beta_{5}$ & $-0,2143$ & 0,1527 & $-0,2964$ & 0,1413 & $-0,2843$ & 0,1326 & $-0,2997$ & 0,1299 \\
$\sigma^{2}$ & 20,9401 & - & 10,6379 & - & 8,6556 & - & 11,1690 & - \\
$\nu$ & - & - & 4,2000 & - & 2,1000 & - & - & - \\
$\gamma$ & - & - & - & - & - & - & 0,1000 & - \\
$\xi$ & - & - & - & - & - & - & 0,1000 & - \\
\hline
\end{tabular}

Tabela 2.3: Critérios de seleção de modelos.

\begin{tabular}{crrrr}
\hline Criterios & N-CR & T- CR & SL-CR & CN-CR \\
\hline log-veross & $-1481,6550$ & $-1440,1450$ & $-1439,5370$ & $-1432,0850$ \\
AIC & 2975,3110 & 2894,2910 & 2893,0750 & 2880,1710 \\
BIC & 3003,0550 & 2926,6590 & 2925,4430 & 2917,1630 \\
EDC & 2996,2400 & 2918,7080 & 2925,4430 & 2908,0760 \\
\hline
\end{tabular}

\subsubsection{Análise de resíduos}

Com o objetivo de verificar se existe alguma especificação incorreta nas suposições do modelo ajustado são mostrados na Figura 2.3 os gráficos dos envelopes simulados para os resíduos martingais transformados. Esta figura indica, que os modelos SMN-CR com caudas mais pesadas são, aparentemente, mais indicados para o ajuste destes dados do que o modelo N-CR, uma vez que há poucas observações que se encontram fora dos envelopes. 

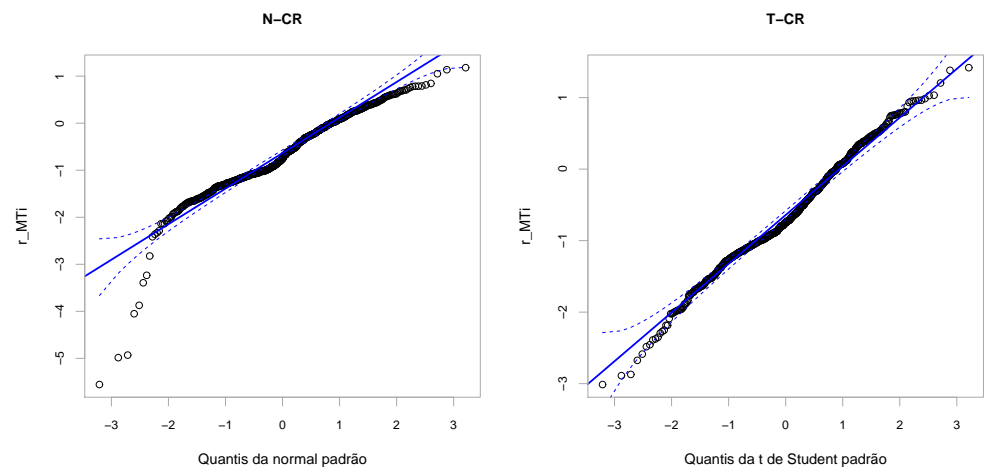

SL-CR
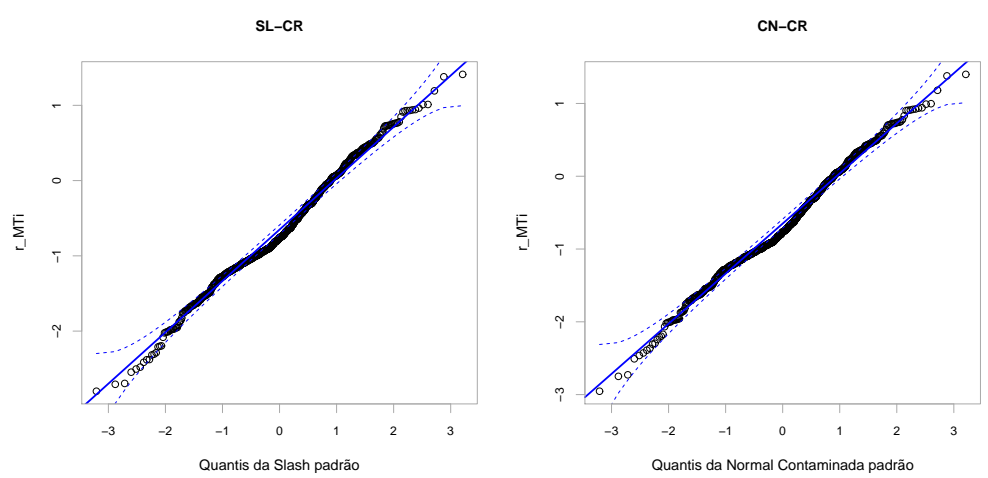

Figura 2.3: Envelopes dos resíduos martingais transformados $r_{M T_{i}}$, para os modelos SMNCR.

\subsubsection{Robustez das estimativas de MV}

A robustez dos três modelos T-CR, S-CR e CN-CR, com caudas mais pesadas que a do modelo N-CR, pode ser avaliada através da influência de uma observação outlier nas estimativas de MV de $\boldsymbol{\theta}$. Em particular, podemos calcular as mudanças relativas nas estimativas de MV de $\boldsymbol{\theta}$ quando uma observação é perturbada em $\nabla$ unidades. Para isto, substituímos o valor da variável $y_{i}$ por $y_{i}(\nabla)=y_{i}+\nabla$.

A mudança relativa é definida por:

$$
R C\left(\beta_{i}\right)=\left|\left(\widehat{\beta}_{i}(\nabla)-\widehat{\beta}_{i}\right) / \widehat{\beta}_{i}\right|, \quad i=1, \ldots, 5,
$$

em que $\widehat{\beta}_{i}(\nabla)$ representa as estimativas de MV de $\beta_{i}$, depois da perturbação.

Neste estudo perturbamos a observação censurada $y_{750}$ e a observação não censurada $y_{7}$, considerando $\nabla=\{0,1, \ldots, 10\}$. A Figura 2.4 mostra os resultados das mudanças relativas das estimativas de MV para os diferentes valores de $\nabla$. Omitimos o gráfico referente a $\beta_{2}$, já que as mudanças relativas são similares para os diferentes modelos. No entanto para os outros parâmetros, como esperado, as estimativas de MV dos modelos com caudas mais pesadas são menos afetadas pelas variações de $\nabla$, independentemente das observações serem censuradas ou não. Assim, temos que os modelos SMN-CR com caudas pesadas são mais robustos, fornecendo estimativas mais precisas quando os dados contêm observações outliers. 
$\beta_{3}$

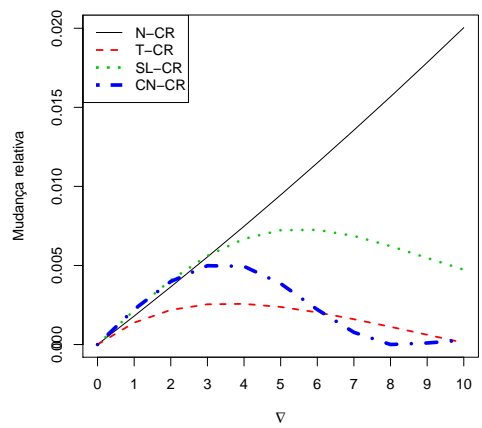

$\beta_{3}$

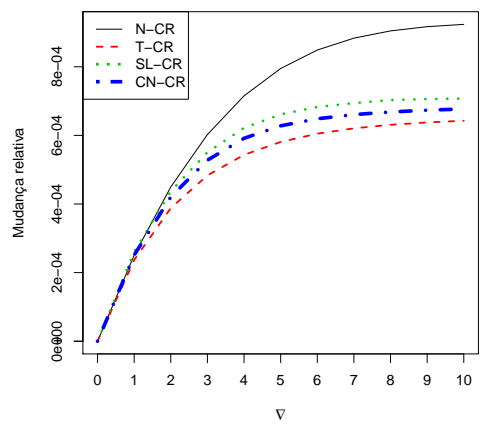

$\beta_{4}$

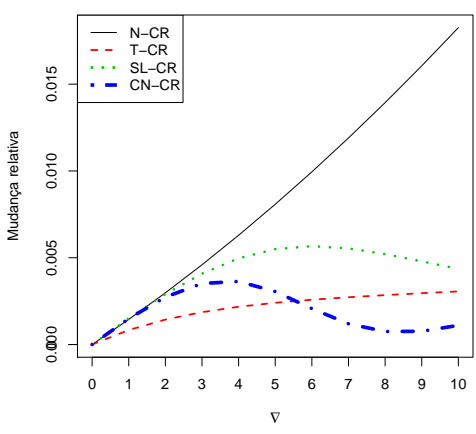

$\beta_{4}$

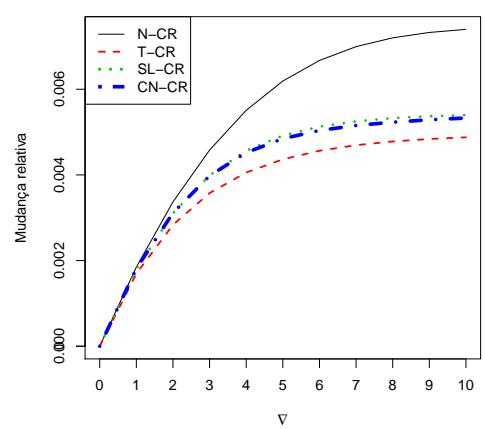

$\beta_{5}$

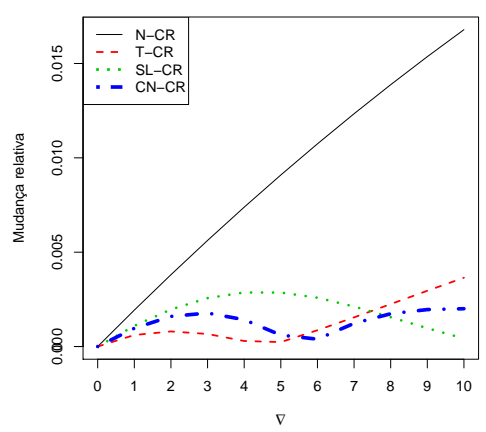

$\beta_{5}$

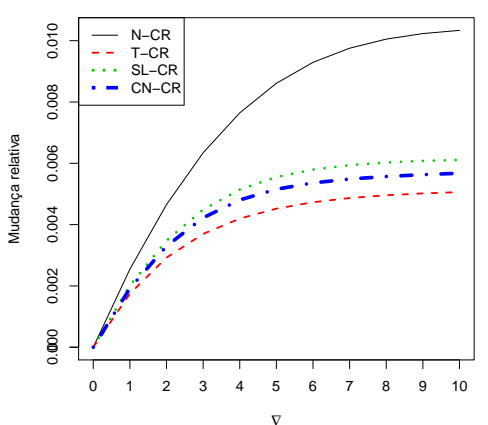

Figura 2.4: Mudanças relativas nas estimativas de MV dos modelos SMN-CR para diferentes contaminações $\nabla$ das observações $y_{7}\left(1^{a}\right.$ linha) e $y_{750}\left(2^{a}\right.$ linha).

O Capítulo 3 é desenvolvido com o intuito de estudar os modelos lineares censurados, sob a classe de distribuições SMN, adotando o enfoque Bayesiano e aplicando as ferramentas apresentadas no Capítulo 1 


\section{Capítulo 3}

\section{Estimação Bayesiana dos modelos SMN-CR}

\subsection{Introdução}

A inferência Bayesiana vem ganhando aceitação como método alternativo devido ao sucesso na solução de problemas complexos em diferentes áreas de interesse, com algoritmos computacionais cada vez mais sofisticados e eficientes. Assim, os métodos numéricos de computação intensiva do tipo Monte Carlo, via Cadeias de Markow (MCMC), apresentados por Gamerman \& Lopes (2006), fornecem uma alternativa eficiente para o tratamento de cálculos bayesianos complicados. Talvez, o método MCMC mais disseminado seja o amostrador de Gibbs, proposto por Geman \& Geman (1984) e adaptado com sucesso para a comunidade estatística por Gelfand et al. (1990). Este método permite obter uma amostra da distribuição a posteriori, sem conhecimento de sua constante normalizadora e, com a amostra gerada, é possível fazer inferência através das integrais de Monte Carlo necessárias. Para mais referências, veja os livros Robert \& Casella (2004), e o já citado Gamerman \& Lopes (2006).

Nesta parte do trabalho estudaremos os modelos SMN-CR a partir de uma perspectiva Bayesiana, para os quais sugerimos um eficiente algoritmo tipo Gibbs com um passo Metropolis-Hastings a fim de obter inferências Bayesianas a posteriori e propomos algumas medidas de diagnóstico Bayesianas baseadas nas medidas de divergências $q$, como foi proposto por Peng \& Dey (1995) e Lachos et al. (2013) para detectar observações influentes. Todas estes procedimentos metodológicos tem sido codificados e implementados na library BayesCR (Garay \& Lachos, 2013), a qual está disponível no repositório CRAN e pode ser instalada livremente. 


\subsection{Inferência Bayesiana nos modelos SMN-CR}

No que segue, para algum vetor $\mathbf{X}$ e $\mathbf{Y}$, usaremos $\pi(\mathbf{x})$ para denotar a densidade de $\mathbf{X}$ e $\pi(\mathbf{x} \mid \mathbf{y})$ para denotar a densidade condicional de $\mathbf{X} \mid \mathbf{Y}=\mathbf{y}$ que, apesar de ser um abuso de notação, simplifica na escrita do conteúdo a seguir.

\subsubsection{Distribuições a priori}

No contexto Bayesiano, a especificação da distribuição a priori para os parâmetros é necessária para obter as inferências a posteriori. Assim, precisamos definir distribuições $a$ priori para todos os parâmetros do modelo SMN-CR, definido em (2.1) e (2.2), isto é $\boldsymbol{\beta}, \sigma^{2}$ e $\boldsymbol{\nu}$. Considerando que não temos informação histórica ou resultados de experimentos prévios relacionados aos dados, vamos supor distribuições a priori próprias com hiperparâmetros conhecidos, mas fracamente informativas para garantir que a distribuição a posteriori seja própria. Desta forma, assumimos que $\boldsymbol{\beta} \sim \mathrm{N}_{p}\left(\mathbf{b}_{0}, \mathbf{S}_{\beta}\right)$, em que $\mathbf{b}_{0}$ é o vetor $p \times 1$ de hiperparâmetros fixados e $\mathbf{S}_{\beta}$ é uma matriz definida positiva conhecida, de ordem $p \times p$. Supomos também, que o parâmetro de escala $\sigma^{2}$ segue uma distribuição inversa gamma, isto é, $\sigma^{-2} \sim \operatorname{Gamma}(a / 2, b / 2)$, em que $a>0$ e $b>0$ são conhecidos.

Cada distribuição da classe SMN tem um parâmetro de fator de escala com interpretação específica e, obviamente, merece um tratamento diferente. Por exemplo, existem muitas sugestões para o caso dos graus de liberdade da distribuição t de Student. Neste sentido, excelentes discussões são apresentadas em Geweke (1993), Fonseca et al. (2008) e Cabral et al. (2012).

Neste texto, utilizaremos a sugestão dada por Cabral et al. (2012), isto é, $\nu \sim \operatorname{Exp}(\lambda)$ com um segundo nível de hierarquia para $\lambda$, dada por $\lambda \sim \mathrm{U}(c, d)$, em que $\operatorname{Exp}(\lambda)$ denota a distribuição exponencial com média $1 / \lambda(\lambda>0)$ e $\mathrm{U}(c, d)$ denota a distribuição Uniforme definida no intervalo $(c, d)$.

Para os graus de liberdade da distribuição slash, consideraremos a mesma sugestão dada por Cabral et al. (2012) para o parâmetro $\nu$ da distribuição t de Student, ou seja, $\nu \sim \operatorname{Exp}(\lambda)$ e $\lambda \sim \mathrm{U}(e, f)$, em que $e$ e $f$ são valores positivos conhecidos.

Para a distribuição normal contaminada, consideraremos como distribuição a priori $\nu \sim \operatorname{Beta}\left(\nu_{0}, \nu_{1}\right)$ e $\gamma \sim \operatorname{Beta}\left(\gamma_{0}, \gamma_{1}\right)$, em que $\nu_{0}, \nu_{1}, \gamma_{0}, \gamma_{1}$ são valores positivos conhecidos. Assim, assumindo independência entre os parâmetros do modelo, a estrutura a priori completa é dada por:

$$
\pi(\boldsymbol{\theta})=\pi(\boldsymbol{\beta}) \pi\left(\sigma^{2}\right) \pi(\boldsymbol{\nu})
$$

\subsubsection{Estimação via MCMC}

No contexto Bayesiano, os estimadores são obtidos a partir de medidas resumo da distribuição a posteriori como, por exemplo, esperança, moda, variância, etc. No entanto, devido a sua forma complexa, não é fácil aproximar estes momentos utilizando técnicas de integração numérica. Atualmente, uma forma eficiente e muito utilizada para aproximar 
estas integrais é através da geração de amostras da distribuição a posteriori, via algoritmo tipo MCMC.

Para nosso modelo SMN-CR, este algoritmo pode ser facilmente desenvolvido utilizando o procedimento de "aumentar" o conjunto de dados, como foi feito para o desenvolvimento do algoritmo tipo EM. Assim, é suficiente obter a distribuição condicional completa de cada parâmetro no modelo, isto é, a distribuição de cada um dos parâmetros dado os dados restantes (incluindo os dados observados).

Seja $Y_{i}$ como definido em (2.1). Então

$$
\begin{gathered}
Y_{i} \mid U_{i}=u_{i} \sim \mathrm{N}\left(\mathbf{x}_{i}^{\top} \boldsymbol{\beta}, u_{i}^{-1} \sigma^{2}\right), \\
U_{i} \sim H(\cdot \mid \boldsymbol{\nu}), \quad i=1, \ldots, n .
\end{gathered}
$$

É importante notar que, considerando os $m$ valores censurados, existe então $m$ valores não observados da característica de interesse, os quais são agrupados no vetor $\mathbf{y}_{L}=$ $\left(y_{1}, \ldots, y_{m}\right)^{\top}$. Obviamente, a ordem dos elementos de $\mathbf{y}_{L}$ é irrelevante e estabelecido unicamente por conveniência. Assim, $y_{i}$ é a realização da variável latente não observada $Y_{i} \sim \operatorname{SMN}\left(\mathbf{x}_{i}^{\top} \boldsymbol{\beta}, \sigma^{2}, \boldsymbol{\nu}\right), i=1, \ldots, m$.

O procedimento chave de nosso algoritmo tipo MCMC é considerar os dados "aumentados" $\left\{\mathbf{y}_{\text {obs }}, \mathbf{y}_{L}, \mathbf{u}\right\}$, em que $\mathbf{u}=\left(u_{1}, \ldots, u_{n}\right)^{\top}$, isto é, considerar o problema como se $\mathbf{y}_{L} \mathrm{e}$ $\mathbf{u}$ fossem de fato observados.

No caso das observações não censuradas, não existe dificuldade para obter as distribuições condicionais. Portanto, é suficiente observar que, dado o valor de $Y_{i}=y_{i}$, então temos que $Y_{\mathrm{obs}_{i}}=y_{i}$.

O algoritmo tem os seguintes passos:

Passo 1. Para cada $i=1, \ldots, m$, gerar observações independentes de $Y_{i}$, a partir da distribuição condicional completa $\pi\left(y_{i} \mid y_{\mathrm{obs}_{i}}, u_{i}, \boldsymbol{\beta}, \sigma^{2}, \boldsymbol{\nu}\right)$, que é uma distribuição normal truncada da forma

$$
\mathrm{NT}\left(\mathbf{x}_{i}^{T} \boldsymbol{\beta}, u_{i}^{-1} \sigma^{2} ;\left\lfloor-\infty, \kappa_{i}\right\rfloor\right) .
$$

Assim, o novo vetor $\mathbf{y}_{\mathrm{obs}}=\left(y_{1}, \ldots, y_{m}, y_{m+1}, \ldots, y_{n}\right)$ está composto pelas observações geradas para os $m$ casos censurados, e os $n-m$ valores observados $y_{i}, i=m+1, \ldots, n$ para os casos não censurados.

Passo 2. Para $i=1, \ldots, n$, gerar observações independentes de $U_{i}$ da distribuição condicional completa $\pi\left(u_{i} \mid y_{\mathrm{obs}_{i}}, \boldsymbol{\beta}, \sigma^{2}, \boldsymbol{\nu}\right)$, que varia segundo as diferentes distribuições SMN da seguinte forma:

(a) para a distribuição t de Student,

$$
\operatorname{Gamma}\left(\frac{\nu+1}{2}, \frac{\left(y_{\mathrm{obs}_{i}}-\mathbf{x}_{i}^{\top} \boldsymbol{\beta}\right)^{2}}{2 \sigma^{2}}+\frac{\nu}{2}\right)
$$


(b) para a distribuição slash,

$$
\text { TGamma }\left(\nu+\frac{1}{2}, \frac{\left(y_{\mathrm{obs}_{i}}-\mathbf{x}_{i}^{\top} \boldsymbol{\beta}\right)^{2}}{2 \sigma^{2}}+\frac{\nu}{2} ;\lfloor 0,1\rfloor\right),
$$

em que TGamma representa a distribuição gamma truncada;

(c) para a distribuição normal contaminada, temos a distribuição discreta que toma os valores de $\gamma$ com probabilidade $\frac{p_{1}^{*}}{p_{1}^{*}+p_{2}^{*}}$ e 1 com probabilidade $\frac{p_{2}^{*}}{p_{1}^{*}+p_{2}^{*}}$, em que

$$
\begin{aligned}
& p_{1}^{*}=\nu \gamma^{(1 / 2)} \exp \left(-\frac{\gamma}{2}\left(\frac{y_{\mathrm{obs}_{i}}-\mathbf{x}_{i}^{\top} \boldsymbol{\beta}}{\sigma}\right)^{2}\right) \mathrm{e} \\
& p_{2}^{*}=(1-\nu) \exp \left(-\frac{1}{2}\left(\frac{y_{\mathrm{obs}_{i}}-\mathbf{x}_{i}^{\top} \boldsymbol{\beta}}{\sigma}\right)^{2}\right) .
\end{aligned}
$$

Passo 3. Gerar observações de $\boldsymbol{\beta}$ a partir da distribuição condicional completa $\pi\left(\boldsymbol{\beta} \mid \mathbf{y}_{\mathrm{obs}}, \mathbf{u}, \sigma^{2}, \boldsymbol{\nu}\right)$, que é definida por

$$
\mathrm{N}_{p}\left(\mathbf{A}_{\beta}\left[\mathbf{S}_{\beta}^{-1} \mathbf{b}_{0}+\sigma^{-2}\left(\sum_{i=1}^{n} u_{i} y_{\mathrm{obs}_{i}} \mathbf{x}_{i}\right)\right], \mathbf{A}_{\beta}\right),
$$

em que $\mathbf{A}_{\beta}=\left(\mathbf{S}_{\beta}^{-1}+\sigma^{-2} \sum_{i=1}^{n} u_{i} \mathbf{x}_{i} \mathbf{x}_{i}^{\top}\right)^{-1}$.

Passo 4. Gerar observações de $\sigma^{-2}$ a partir da distribuição condicional completa $\pi\left(\sigma^{-2} \mid \mathbf{y}_{\text {obs }}, \mathbf{u}, \boldsymbol{\beta}, \nu, \lambda\right)$, que é definida por

$$
\operatorname{Gamma}\left(\frac{n+a}{2}, \frac{b+\sum_{i=1}^{n} u_{i}\left(y_{\mathrm{obs}_{i}}-\mathbf{x}_{i}^{\top} \boldsymbol{\beta}\right)^{2}}{2}\right) .
$$

Passo 5. Gerar observações de $\boldsymbol{\nu}$ a partir da distribuição condicional completa. Este procedimento de geração depende da distribuição SMN escolhida, e em alguns casos é necessário introduzir um passo Metropolis-Hastings.

Assim, por exemplo,

(a) para a distribuição t de Student,

i. gerar observações de $\lambda$ a partir da distribuição condicional $\pi(\lambda \mid \nu)$, a qual é $\operatorname{TGamma}(2, \nu ;\lfloor c, d\rfloor)$.

ii. utilizando o método Metropolis-Hastings, gerar observações de $\nu$ a partir da distribuição condicional marginal

$$
\begin{aligned}
& \pi\left(\nu \mid \mathbf{y}_{\mathrm{obs}}, \boldsymbol{\beta}, \sigma^{2}, \lambda\right) \propto \exp (-\lambda \nu) \times \\
& {\left[\sum_{i=1}^{m} \mathrm{~T}_{\nu}\left(\frac{\kappa_{i}-\mathbf{x}_{i}^{\top} \boldsymbol{\beta}}{\sigma}\right)+\sum_{i=m+1}^{n} \mathrm{t}_{\nu}\left(y_{i} \mid \mathbf{x}_{i}^{\top} \boldsymbol{\beta}, \sigma^{2}\right)\right] .}
\end{aligned}
$$

As propostas são obtidas da seguinte forma: Dada uma observação $\nu^{(j-1)}$ obtida na fase $j-1$, geramos uma observação candidata $\nu^{*}$ da distribuição Log-normal

$$
\mathrm{LN}\left(\log \nu^{(j-1)}, \delta_{\nu}^{2}\right)
$$


cuja fdp é definida por:

$$
q\left(x ; \mu, \sigma^{2}\right)=\frac{1}{x \sigma \sqrt{(2 \pi)}} \exp \left\{-\frac{(\ln (x)-\mu)^{2}}{2 \sigma^{2}}\right\} .
$$

Assim, calculando

$$
\frac{q\left(\nu^{*} \mid \log \nu^{(j-1)}, \delta_{\nu}^{2}\right)}{q\left(\nu^{(j-1) \mid \log \nu^{*}}, \delta_{\nu}^{2}\right)}=\frac{\nu^{(j-1)}}{\nu^{*}},
$$

temos que a nova observação $\nu^{*}$ é aceita com probabilidade

$$
\min \left\{\frac{\pi\left(\nu^{*} \mid \cdots\right) \nu^{(j-1)}}{\pi\left(\nu^{(j-1)} \mid \cdots\right) \nu^{*}}, 1\right\}
$$

em que $\pi\left(\nu^{*} \mid \cdots\right)$ representa a distribuição condicional marginal (3.2), avaliada utilizando os valores atuais de $\boldsymbol{\beta}, \sigma^{2}, \lambda$ e $\mathbf{y}_{\text {obs. }}$. Neste caso, as amostras são obtidas a partir das distribuições parcialmente marginais, integrando as variáveis latentes $u_{1}, \ldots, u_{n}$. Este método, conhecido como principio collapsing, geralmente é mais eficiente do que a amostragem da distribuição condicional completa. Veja Liu (1994) para uma discussão mais detalhada;

(b) para a distribuição slash

i. gerar observações de $\lambda$ a partir da distribuição condicional $\pi(\lambda \mid \nu)$, a qual é $\operatorname{TGamma}(2, \nu ;\lfloor e, f\rfloor)$.

ii. gerar observações de $\nu$ a partir da distribuição condicional $\pi(\nu \mid \mathbf{u})$ que é definida por

$$
\operatorname{Gamma}\left(n+1, \lambda-\sum_{i=1}^{n} \log \left(u_{i}\right)\right) ;
$$

(c) para a distribuição normal contaminada

i. gerar uma amostra de $\nu$ a partir da distribuição condicional $\pi(\nu \mid \mathbf{u}, \gamma)$, que é Beta $\left(\nu_{0}+m_{\gamma} ; \nu_{1}+n-m_{\gamma}\right)$, em que $m_{\gamma}=\left(n-\sum_{i=1}^{n} \mathrm{~S}_{i}\right) /(1-\gamma)$ e

$$
S_{i}=\left\{\begin{array}{ccc}
1 & \text { se } & u_{i}=\gamma \\
0 & \text { se } & u_{i} \neq \gamma
\end{array}\right.
$$

ii. considere a distribuição condicional marginal de $\gamma \mid \mathbf{y}_{\text {obs }}, \boldsymbol{\beta}, \sigma^{2}, \nu$, definida por

$$
\begin{aligned}
& \pi\left(\gamma \mid \mathbf{y}_{\text {obs }}, \boldsymbol{\beta}, \sigma^{2}, \nu\right) \propto \gamma^{\gamma_{0}-1}(1-\gamma)^{\gamma_{1}-1} \times \\
& {\left[\sum_{i=1}^{m} F_{C N}\left(\frac{\kappa_{i}-\mathbf{x}_{i}^{\top} \boldsymbol{\beta}}{\sigma}, \nu, \gamma\right)+\sum_{i=m+1}^{n} f_{C N}\left(y_{i} \mid \mathbf{x}_{i}^{\top} \boldsymbol{\beta}, \sigma^{2}\right)\right],}
\end{aligned}
$$

em que $F_{C N}$ é a fda da normal contaminada.

Dada a parametrização $\gamma_{r}=\gamma / 1-\gamma$, gerar observações $\gamma_{r}$ da distribuição $\pi\left(\gamma_{r} \mid \mathbf{y}_{\text {obs }}, \boldsymbol{\beta}, \sigma^{2}, \nu\right)=\frac{1}{\left(1+\gamma_{r}\right)^{2}} \pi\left(\frac{\gamma_{r}}{1+\gamma_{r}} \mid \mathbf{y}_{\text {obs }}, \boldsymbol{\beta}, \sigma^{2}, \nu\right)$, utilizando o método de Metropolis-Hastings com a distribuição Lognormal, como densidade proposta. 
Assim, dada uma observação $\gamma_{r}^{j-1}$ obtida na fase $j-1$, gerar uma observação candidata $\gamma_{r}^{*}$ a partir da distribuição $\operatorname{LN}\left(\log \gamma_{r}^{(j-1)}, \delta_{\gamma_{r}}^{2}\right)$.

Considerando a equação (3.3), teremos que, a nova observação $\gamma^{*}$ é gerada com probabilidade

$$
\min \left\{\frac{\pi\left(\gamma^{*} \mid \cdots\right) \gamma^{(j-1)}}{\pi\left(\gamma^{(j-1)} \mid \cdots\right) \gamma^{*}}, 1\right\} .
$$

Neste caso, as amostras são obtidas a partir de distribuições parcialmente marginais, integrando as variáveis latentes $u_{1}, \ldots, u_{n}$.

\subsection{Critérios de comparação de modelos}

$\mathrm{Na}$ literatura, encontramos diversas metodologias que são utilizadas para analisar a adequabilidade de um modelo, além de, dentre uma coleção de modelos selecionar o mais adequado no ajuste para um conjunto de dados. Veja Ando (2010) para maiores detalhes.

Um dos critérios mais utilizados em trabalhos aplicados é obtido através da estatística preditiva condicional ordenada (CPO). Assim, seja $\mathbf{z}=\left\{z_{1}, \ldots, z_{n}\right\}$ uma amostra observada de $\pi(\cdot \mid \boldsymbol{\theta})$. Temos que para a $i$-ésima observação, o $\mathrm{CPO}_{i}$ pode ser escrito como

$$
\mathrm{CPO}_{i}=\int \pi\left(z_{i} \mid \boldsymbol{\theta}\right) \pi\left(\boldsymbol{\theta} \mid \mathbf{z}_{(-i)}\right) d \boldsymbol{\theta}=\left(\int \frac{\pi(\boldsymbol{\theta} \mid \mathbf{z})}{\pi\left(z_{i} \mid \boldsymbol{\theta}\right)} d \boldsymbol{\theta}\right)^{-1}
$$

em que $\mathbf{z}_{(-i)}$ é a amostra observada sem a $i$-ésima observação.

Para nosso modelo censurado SMN-CR, descrito em (2.1) e (2.2), o $\mathrm{CPO}_{i}$ não tem uma expressão com forma fechada, no entanto podemos obter uma estimativa Monte Carlo do $\mathrm{CPO}_{i}$ através da amostra MCMC $\boldsymbol{\theta}_{1}, \ldots, \boldsymbol{\theta}_{Q}$ da distribuição a posteriori $\pi(\boldsymbol{\theta} \mid \mathbf{z})$. Como foi mostrado em Dey et al. (1997), esta aproximação é definida por

$$
\widehat{\mathrm{CPO}_{i}}=\left(\frac{1}{Q} \sum_{q=1}^{Q} \frac{1}{\pi\left(z_{i} \mid \boldsymbol{\theta}_{q}\right)}\right)^{-1} .
$$

Uma estatística relacionada com o $\mathrm{CPO}_{i}$ comumente utilizada é a Pseudo Log-verossimilhança marginal (LPML), definida por

$$
\mathrm{LPML}=\sum_{i=1}^{n} \log \left(\widehat{\mathrm{CPO}_{i}}\right) .
$$

Quanto maior é o valor de LMPL, melhor é o ajuste.

Utilizando o LPML podemos estimar o Pseudo fator de Bayes (PBF), utilizado para comparar dois modelos $M_{0}$ e $M_{1}$, o qual é definido por

$$
\operatorname{PBF}\left(M_{0}, M_{1}\right)=\exp \left(\mathrm{LPML}_{0}-\mathrm{LPML}_{1}\right)
$$

em que $\mathrm{LMPL}_{i}$ é a log-pseudo verosimilhança marginal para o modelo $M_{i}, i=0,1$. 
O critério de informação do desvio (DIC) é outra medida de qualidade do ajuste e de complexidade do modelo, comumente utilizada na literatura estatística para seleção de modelos Bayesianos, o qual é definido por

$$
\mathrm{DIC}=\overline{\mathrm{D}}(\boldsymbol{\theta})+\rho_{\mathrm{D}},
$$

em que o primeiro termo representa a esperança a posteriori do desvio, dada por $\overline{\mathrm{D}}(\boldsymbol{\theta})=$ $-2 \mathrm{E}[\log \pi(\mathbf{z} \mid \boldsymbol{\theta}) \mid \mathbf{z}]$, e o segundo termo $\rho_{D}$ representa o número efetivo de parâmetros, definido como $\mathrm{E}[\mathrm{D}(\boldsymbol{\theta}) \mid \mathbf{z}]-\mathrm{D}(\mathrm{E}[\boldsymbol{\theta} \mid \mathbf{z}])$, em que $\mathrm{D}(\boldsymbol{\theta})=-2 \log \pi(\mathbf{z} \mid \boldsymbol{\theta}), \mathrm{E}[D(\boldsymbol{\theta}) \mid \mathbf{z}]$ é a média a posteriori do desvio e $\mathrm{D}(\mathrm{E}[\boldsymbol{\theta} \mid \mathbf{z}])$ é o desvio avaliado na média a posteriori.

A média a posteriori do desvio pode ser aproximada utilizando a amostra MCMC da seguinte forma: $\bar{D}=\sum_{q=1}^{Q} D\left(\boldsymbol{\theta}_{q}\right) / Q$, e o desvio avaliado na média a posteriori pode também ser aproximado por $D\left(\frac{1}{Q} \sum_{q=1}^{Q} \boldsymbol{\theta}_{q}\right)$, considerando que uma estimativa Monte Carlo de $\mathrm{E}[\boldsymbol{\theta} \mid \mathbf{y}]$ é $\frac{1}{Q} \sum_{q=1}^{Q} \boldsymbol{\theta}_{q}$, em que $\boldsymbol{\theta}_{1}, \ldots, \boldsymbol{\theta}_{q}$ é a amostra MCMC da distribuição a posteriori $\pi(\boldsymbol{\theta} \mid \mathbf{y})$. Finalmente, o DIC pode ser estimado por

$$
\widehat{\mathrm{DIC}}=2 \overline{\mathrm{D}}-\mathrm{D}\left(\frac{1}{Q} \sum_{q=1}^{Q} \boldsymbol{\theta}_{q}\right)
$$

Segundo o critério DIC, ao comparar-se o ajuste de dois modelos alternativos para um determinado conjunto de dados, o modelo melhor ajuste é aquele que apresenta menor valor DIC. Outras propriedades do DIC podem ser encontradas em Spiegelhalter et al. (2002).

Como mencionado por Chen et al. (2008), o CPO e o DIC são métodos baseados no critério preditivo a posteriori, os quais são atrativos no sentido de serem bem definidos sob distribuições a priori impróprias e/ou vagas próprias.

Outros critérios que podem ser utilizados para comparar modelos são o Critério Esperado de informação de Akaike (Brooks, 2002), e o Critério Esperado de Informação Bayesiano (EBIC) (Carlin \& Louis, 2001). De forma similar, estes critérios podem ser estimados por

$$
\widehat{E A I C}=\overline{\mathrm{D}}+2 \vartheta \quad \text { e } \widehat{E B I C}=\overline{\mathrm{D}}+\vartheta \log (n)
$$

em que $\vartheta$ é o número de parâmetros no modelo.

Para determinar se um modelo é adequado, utilizamos a medida de discrepância baseada na distribuição preditiva. Se o valor observado for extremo em referência à distribuição preditiva, existe indícios de problemas no ajuste do modelo para um determinado conjunto de dados.

Seja $y_{i}, \quad i=1, \ldots, n$ os dados observados. Então, a medida de discrepância entre o modelo e dados é obtido como uma estatística resumo, introduzida por Gelman et al. (2004), que é definida por

$$
T(y, \boldsymbol{\theta})=-2 \sum_{i=1}^{n} \log \left[\pi\left(y_{i} \mid \boldsymbol{\theta}\right)\right] .
$$


O p-valor Bayesiano ou p-valor preditivo a posteriori $\mathrm{p}_{\mathrm{B}}$ (Gelman et al., 2004) é definido como o número de vezes que $T\left(y_{p r}, \boldsymbol{\theta}\right)$ excede $T(y, \boldsymbol{\theta})$ a partir de um número $L$ de amostras geradas, isto é, $p_{B}=\operatorname{Pr}\left(T\left(y_{p r}, \boldsymbol{\theta}\right) \geq T(y, \boldsymbol{\theta}) \mid Y=y\right)$, em que $y_{p r}$ denota uma amostra simulada da distribuição preditiva a posteriori. Um valor grande $(>0,95)$ ou um valor muito pequeno $(<0,05)$ de $\mathrm{p}_{\mathrm{B}}$ é um indicio de má especificação do modelo revelando que o padrão observado seria impossível de ser visto em repetições dos dados sob o modelo verdadeiro.

\subsection{Diagnóstico de Influência Bayesiano}

Nesta seção, consideraremos algumas medidas de diagnóstico de influência Bayesiano, baseadas no método de eliminação para determinar observações que são globalmente influentes na análise. Assim, o cálculo das medidas de divergência entre as distribuições $a$ posteriores, com e sem um determinado subconjunto de dados, é uma maneira útil de quantificar a influência.

A medida $q$-divergência entre duas densidades $\pi_{1}(\cdot)$ e $\pi_{2}(\cdot)$, para $\boldsymbol{\theta}$ (Csiszar, 1967) é definida por

$$
d_{q}\left(\pi_{1}, \pi_{2}\right)=\int q\left(\frac{\pi_{1}(\boldsymbol{\theta})}{\pi_{2}(\boldsymbol{\theta})}\right) \pi_{2}(\boldsymbol{\theta}) d \boldsymbol{\theta},
$$

em que $q$ é uma função convexa, tal que $q(1)=0$.

Algumas medidas de divergência específicas são obtidas considerando diferentes tipos de funções para $q(\cdot)$. Por exemplo, a divergência de Kullback-Leibler é obtida quando $q(z)=-\log (z)$; a medida de divergência $J$ (uma versão simétrica da divergência KullbackLeibler) é obtida quado $q(z)=(z-1) \log (z)$ e a medida de divergência $L_{1}$ é obtida quando $q(z)=|z-1|$.

Seja $\mathbf{y}=\left\{y_{1}, \ldots, y_{n}\right\}$ uma amostra e $I$ um subconjunto de $\{1, \ldots, n\}$. Definimos também $\mathbf{y}_{I}=\left\{y_{i} ; i \in I\right\}$ e denotamos $\mathbf{y}_{(-I)}$ como o seu complemento . A $q$-influência de $\mathbf{y}_{I}$ na distribuição a posteriori de $\boldsymbol{\theta}$ é obtida substituindo $\pi_{1}(\boldsymbol{\theta})=\pi_{1}\left(\boldsymbol{\theta} \mid \mathbf{y}_{(-I)}\right)$ e $\pi_{2}(\boldsymbol{\theta})=\pi(\boldsymbol{\theta} \mid \mathbf{y})$ na equação (3.6). Esta medida de influênia pode ser escrita como

$$
d_{q}(I)=\mathrm{E}\left[q\left(\frac{\pi_{1}\left(\boldsymbol{\theta} \mid \mathbf{y}_{(-I)}\right)}{\pi_{2}(\boldsymbol{\theta} \mid \mathbf{y})}\right) \mid \mathbf{y}\right]
$$

É importante destacar que todas estas medidas podem ser aproximadas utilizando amostras a posteriori via MCMC.

Note que é preciso estabelecer um ponto de corte para tais medidas de divergêcia, acima do qual uma observação na amostra é considerada influente para as inferências do modelo. Para isso usaremos a proposta dada por Peng \& Dey (1995) e discutida recentemente por Vidal \& Castro (2010).

Para explicar de forma intuitiva estes pontos de corte, considere X uma variável aleatória que assume o valor 1 caso o lançamento de uma moeda resulte em cara, o que ocorre 
com probabilidade $p$, ou assume o valor 0 , caso contrário. A densidade de $X$ é dada por $\pi_{1}(x \mid p)=p^{x}(1-p)^{1-x}$, com $x=0,1$ e $p \in[0,1]$. Se quisermos ajustar um modelo aos resultados do experimento e assumirmos que a moeda é honesta, temos como suposição que $p=0,5$ e a densidade de $X$ sob esta suposição é dada por $\pi_{2}(x \mid p=1 / 2)=1 / 2$. De (3.6), a divergência $q$ entre a densidade assumida para $X$ (moeda honesta) e a densidade real (dependente do parâmetro $p$ desconhecido) é dada por

$$
d_{q}(p)=\frac{q(2 p)+q(2(1-p))}{2} .
$$

É intuitivo que $d_{q}(p)$ cresce quando $p$ se afasta de 0,5 , que é simétrico ao redor de $p=0,5$ e que alcança seu valor mínimo quando $p=0,5$. Além disso, $d_{q}(p)=0$ se e somente se $p=0,5$, isto é, se o modelo assumido for, de fato, o modelo real: $\pi_{1}=\pi_{2}$. Desta forma, podemos estabelecer por nosso próprio critério que o modelo assumido é diferente do real se $p>0,75$ ou $p<0,25$.

Considerando $\pi_{0}$ o modelo com todas as observações da amostra e $\pi_{i}$ o modelo sem a $i$-ésima observção e assumindo um critério análogo a tomar o valor limitante $p=0,75$ no exemplo acima, podemos afirmar que quando $d_{L_{1}}\left(\pi_{0}, \pi_{i}\right) \geq 0,25, d_{K L}\left(\pi_{0}, \pi_{i}\right)>0,14$ ou $d_{J}\left(\pi_{0}, \pi_{i}\right)>0,27$, a observação $i$ é dada como influente.

\subsection{Estudos de simulação}

Com o intuito de avaliar do desempenho de nosso modelo e algoritmo, apresentamos dois estudos de simulação, que foram implementados utilizando o software R (R Core Team, 2013). Na primeira parte deste estudo de simulação mostramos as consequências na inferência dos parâmetros quando a suposição de normalidade não é apropriada. O objetivo da segunda parte é comparar as estimativas dos graus de liberdade $\nu$, para o modelo T-CR, quando consideramos diferentes distribuições a priori, $\pi(\nu)$, tipicamente usadas na literatura.

Consideramos amostras geradas, de tamanho $n=300$, de um modelo linear censurado, definido em (2.1) e (2.2), em que $\varepsilon_{i} \sim t_{\nu}\left(0, \sigma^{2}\right)$, com $\boldsymbol{\beta}^{\top}=\left(\beta_{1}, \beta_{2}\right)=(1,2), \sigma^{2}=3, \nu=4$, um percentagem de nível de censura fixado $p$ e $\mathbf{x}_{i}^{\top}=\left(1, x_{i 2}\right)$. Cada $x_{i 2}$ é uma amostra aleatória de uma distribuição $U(1,3)$. Esses valores foram fixados para todas as amostras.

\subsubsection{Simulação 1}

O objetivo de este estudo de simulação é avaliar as consequências na inferência dos parâmetros quando a suposição de normalidade é inapropriado considerando diferentes níveis de censuras $p$ (isto é $5 \%, 10 \%, 15 \%, 20 \%, 25 \%, 30 \%$ e $50 \%$ ) e diferentes distribuições a priori pra $\nu$, como visto na Subseção 3.2 .1 , com $\mathbf{b}_{0}^{\top}=\mathbf{0}_{2}, \mathbf{S}_{\beta}=10^{3} \mathbf{I}_{2}, a=2, b=0,02$, $c=0,02$ e $d=0,5$.

Para cada umas das combinações, nível de censura e distribuição a priori pra $\nu$, geramos 400 amostras Monte Carlo e para cada amostra gerada, encontramos as estimativas $a$ 
posteriori dos parâmetros sob os modelos T-CR e N-CR.

Na tabela 3.1 apresentamos as medidas resumo das estimativas de $\boldsymbol{\beta}$ e $\sigma^{2}$, em que MC Média e MC DP denotam o valor médio e o desvio padrão das estimativas, respectivamente, obtidas considerando todas as amostras MC geradas. MC Cov representa percentagem de vezes, considerando o total de amostras geradas, que o intervalo de credibilidade HPD $a$ posteriori de $95 \%$ contêm o verdadeiro valor do parâmetro.

\begin{tabular}{|c|c|c|c|c|c|c|c|}
\hline \multirow[t]{2}{*}{ Censura } & \multirow[t]{2}{*}{ Parâmtros } & \multicolumn{3}{|l|}{ N-CR } & \multicolumn{3}{|l|}{ t-CR } \\
\hline & & MC Média & MC DP & MC Cov & MC Média & MC DP & $\mathrm{MC} \mathrm{Cov}$ \\
\hline \multirow[t]{3}{*}{$5 \%$} & $\beta_{1}$ & 0,9827 & $(0,5049)$ & $95,70 \%$ & 1,0111 & $(0,4125)$ & $97,97 \%$ \\
\hline & $\beta_{2}$ & 1,9918 & $(0,2426)$ & $96,71 \%$ & 1,9925 & $(0,2032)$ & $97,72 \%$ \\
\hline & $\sigma^{2}$ & 5,4371 & $(0,9013)$ & $95,70 \%$ & 2,8588 & $(0,3834)$ & $98,27 \%$ \\
\hline \multirow[t]{3}{*}{$10 \%$} & $\beta_{1}$ & 0,9163 & $(0,5064)$ & $96,96 \%$ & 0,9631 & $(0,4429)$ & $97,47 \%$ \\
\hline & $\beta_{2}$ & 2,0241 & $(0,2358)$ & $97,22 \%$ & 2,0201 & $(0,2111)$ & $98,48 \%$ \\
\hline & $\sigma^{2}$ & 5,4253 & $(1,1646)$ & $94,19 \%$ & 2,8310 & $(0,3701)$ & $96,71 \%$ \\
\hline \multirow[t]{3}{*}{$15 \%$} & $\beta_{1}$ & 0,9018 & $(0,5155)$ & $96,20 \%$ & 0,9803 & $(0,4489)$ & $98,23 \%$ \\
\hline & $\beta_{2}$ & 2,0300 & $(0,2543)$ & $94,94 \%$ & 2,0075 & $(0,2159)$ & $96,71 \%$ \\
\hline & $\sigma^{2}$ & 5,3721 & $(2,5729)$ & $94,34 \%$ & 2,8697 & $(0,3858)$ & $95,80 \%$ \\
\hline \multirow[t]{3}{*}{$20 \%$} & $\beta_{1}$ & 0,8980 & $(0,5596)$ & $94,94 \%$ & 0,9900 & $(0,4826)$ & $97,72 \%$ \\
\hline & $\beta_{2}$ & 2,0381 & $(0,2713)$ & $94,18 \%$ & 2,0034 & $(0,2330)$ & $98,23 \%$ \\
\hline & $\sigma^{2}$ & 5,3144 & $(0,9679)$ & $93,39 \%$ & 2,8643 & $(0,3803)$ & $95,65 \%$ \\
\hline \multirow[t]{3}{*}{$25 \%$} & $\beta_{1}$ & 0,8667 & $(0,5214)$ & $95,19 \%$ & 1,0195 & $(0,4549)$ & $96,20 \%$ \\
\hline & $\beta_{2}$ & 2,0643 & $(0,2549)$ & $95,44 \%$ & 1,9912 & $(0,2217)$ & $97,47 \%$ \\
\hline & $\sigma^{2}$ & 5,4340 & $(1,4972)$ & $95,95 \%$ & 2,8315 & $(0,3910)$ & $96,12 \%$ \\
\hline \multirow[t]{3}{*}{$30 \%$} & $\beta_{1}$ & 0,7985 & $(0,5297)$ & $95,19 \%$ & 0,9960 & $(0,4663)$ & $97,72 \%$ \\
\hline & $\beta_{2}$ & 2,1130 & $(0,2616)$ & $94,43 \%$ & 2,0056 & $(0,2288)$ & $96,96 \%$ \\
\hline & $\sigma^{2}$ & 5,5431 & $(1,2213)$ & $95,70 \%$ & 2,8813 & $(0,3988)$ & $97,42 \%$ \\
\hline \multirow[t]{3}{*}{$50 \%$} & $\beta_{1}$ & 0,7156 & $(0,5925)$ & $94,18 \%$ & 1,0298 & $(0,4949)$ & $95,95 \%$ \\
\hline & $\beta_{2}$ & 2,2311 & $(0,3167)$ & $92,91 \%$ & 1,9890 & $(0,2551)$ & $96,46 \%$ \\
\hline & $\sigma^{2}$ & 6,2252 & $(1,5874)$ & $94,43 \%$ & 2,8859 & $(0,5018)$ & $96,20 \%$ \\
\hline
\end{tabular}

Tabela 3.1: MC média, MC DP (Em parêntese) e MC Cov para os modelos T-CR e N-CR com diferentes níveis de censura.

Da Tabela 3.1, observamos que o modelo T-CR apresentam melhor performance para todos os níveis de censura. A Figura 3.1 mostra que um incremento notório do Viés (Desvio do parâmetro estimado em comparação com o verdadeiro valor do parâmetro) quando usamos a suposição de normalidade para os erros. Claramente, a suposição da distribuição $\mathrm{T}$ de Student produz estimativas mais precisas. Além disso, observa-se na Figura 3.2 que as estimativas a posteriori obtidas sob o modelo T-CR são mais estáveis, especialmente quando os níveis de censura aumentam. Portanto, este estudo de simulação proporciona evidências de que o modelo com cudas mais pesadas do que a distribuição normal produz estimativas a posteriori mais precisas, no contexto dos dados censurados, já que todas as medidas consideradas favoreceram fortemente ao modelo T-CR detectando desvios evidentes da normalidade. 

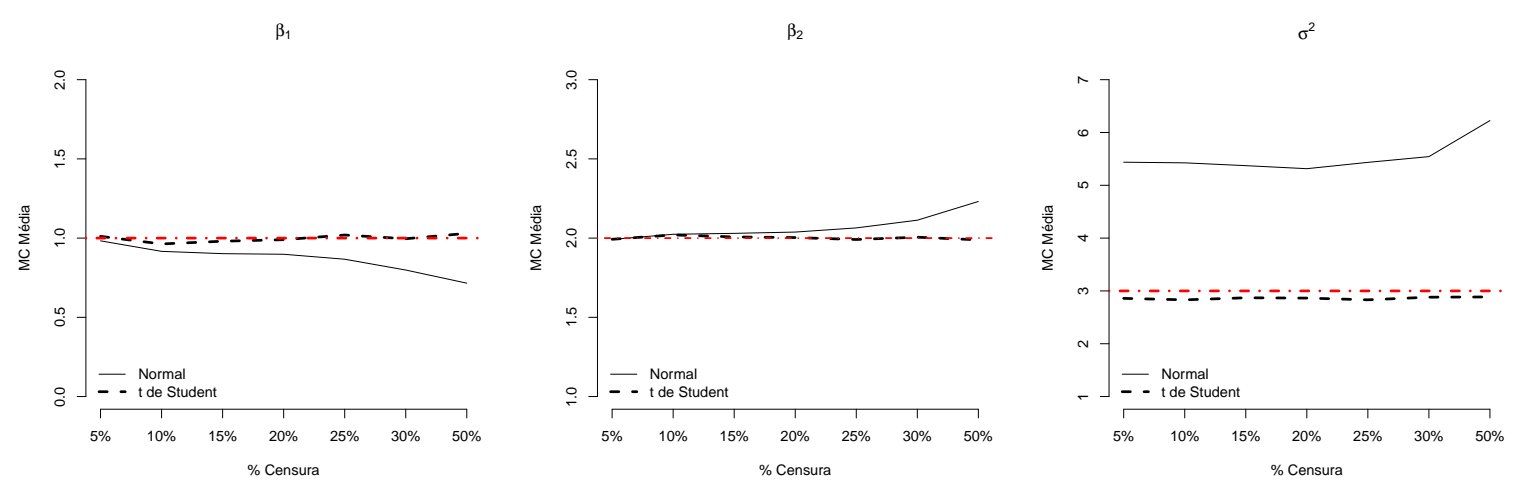

Figura 3.1: MC Média dos parâmetros $\beta_{1}, \beta_{2}$ e $\sigma^{2}$ para os modelos N-CR e T-CR em comparação com o verdadeiro valor dos parâmetros (Linha vermelha)
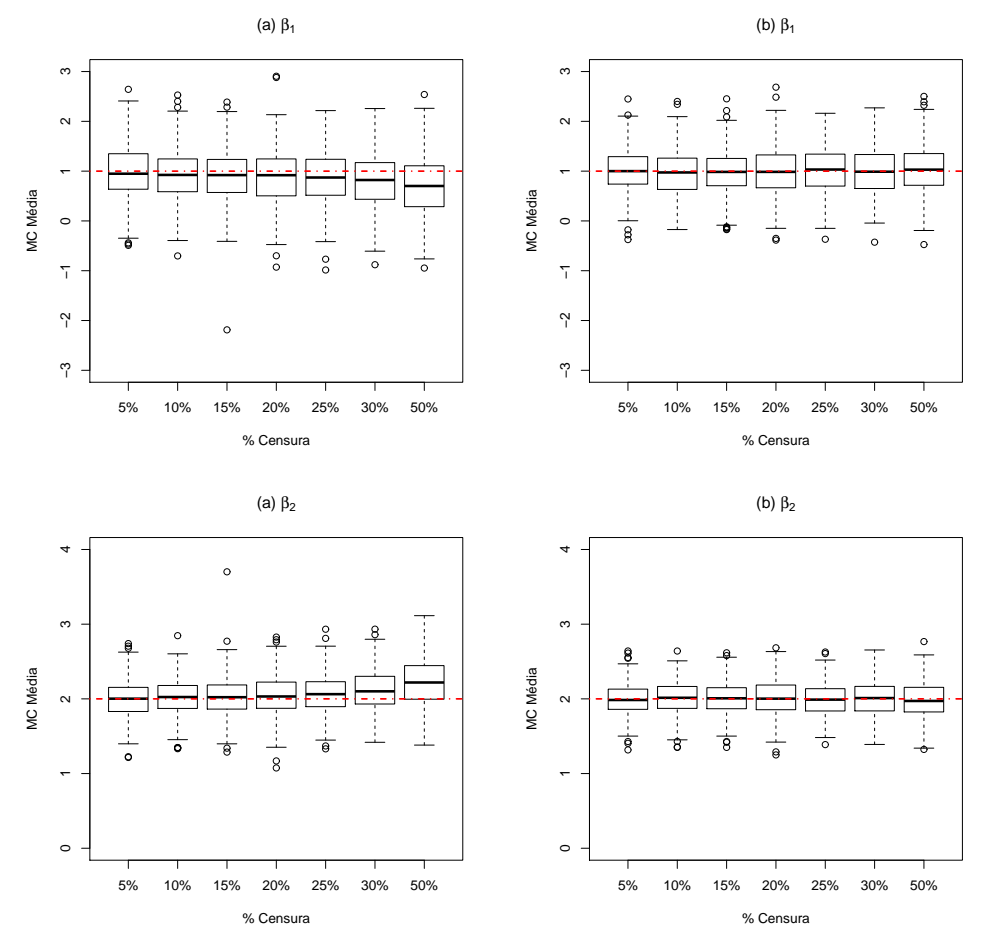

Figura 3.2: Gráfico de caixas do MC Média dos parâmetros $\beta_{1}$ e $\beta_{2}$, sob os modelos (a) N-CR e (b) T-CR. A linha vermelha representa o verdadeiro valor dos parâmetros.

\subsubsection{Simulação 2}

Este estudo de simulação foi desenvolvido com o intuito de avaliar as propriedades frequentistas da estimação de $\nu$, no modelo censurado T-CR, baseadas em diferentes distribuições a priori tipicamente usadas na literatura. Para isto, consideramos o modelo T-CR com $8 \%$ de nível de censura e diferentes valores para $\nu$, i.e, $\nu=4,6,8,10,12,14$ e 20.

Para cada um destes valores, foram geradas $R=400$ amostras Monte Carlo e para efeitos de comparação foram consideradas as seguintes distribuições a priori para $\nu$ : 
(a)

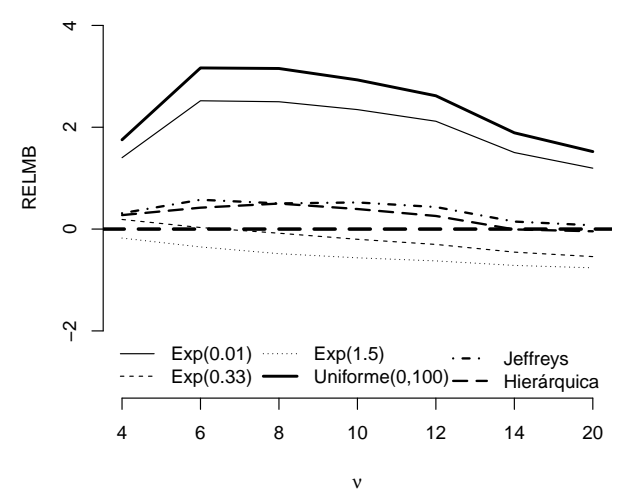

(b)

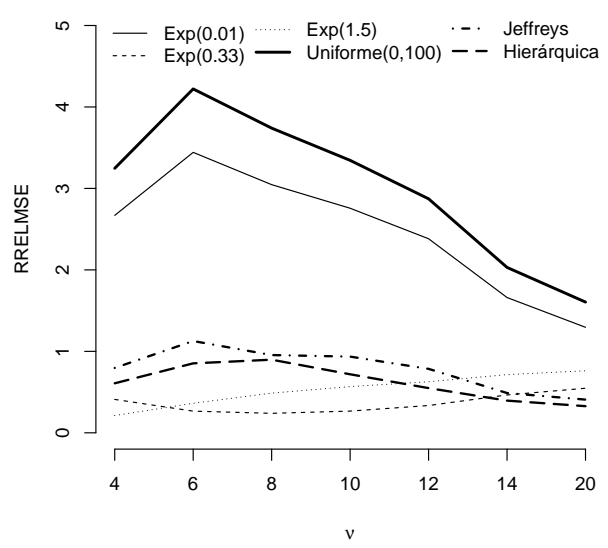

Figura 3.3: Estudo de simulação: (a) RELMB e (b) RRELMSE sob diferentes a priori para $\nu$.

- $\nu \sim \operatorname{Exp}\left(\lambda_{i}\right) \mathbb{I}_{\lfloor 2, \infty\rfloor}(\nu), i=1,2,3$, isto é, a distribuição exponencial truncada em $\lfloor 2, \infty\rfloor$, em que $\lambda_{1}=0,01, \lambda_{2}=0,33$ e $\lambda_{3}=1,5$. Esta proposta foi feita originalmente por Geweke (1993);

- $\nu \sim \mathrm{U}(2,100)$;

- $\pi_{3}(\nu)=\left(\frac{\nu}{\nu+3}\right)^{\frac{1}{2}}\left\{\psi^{\prime}\left(\frac{\nu}{2}\right)-\psi^{\prime}\left(\frac{\nu+1}{2}\right)-\frac{2(\nu+3)}{\nu(\nu+1)^{2}}\right\}^{\frac{1}{2}}$, em que $\psi_{1}(x)=\frac{d^{2}}{d x^{2}} \log \Gamma(x)$ é a função tri gamma. Esta é a distribuição a priori objetiva de Jeffreys proposta por Fonseca et al. (2008);

- A distribuição a priori hierárquica sugerida por Cabral et al. (2012), isto é, $\nu \sim \operatorname{Exp}(\lambda)$ $\operatorname{com} \lambda \sim \mathrm{U}(0,02,0,5)$. A escolha dos valores $\varsigma_{0}=0,02$ e $\varsigma_{1}=0,5$ garante que o valor esperado de $\nu$ se encontre no intervalo $[2,50]$.

Finalmente, consideramos as seguintes distribuições a priori para os outros parâmetros: $\beta_{k} \sim N_{1}\left(0,10^{3}\right)$ para $k=1,2$, e $\sigma^{-2} \sim$ Gamma $(0.1,0.01)$. Todos os resultados são baseados em 50,000 iterações obtidas depois de um burn-in de 10,000 iterações. Como o principal interesse é a estimação do parâmetro $\nu$ sob as quatro distribuições a priori descritas acima, utilizamos o viés relativo (RELMB) e a raiz do erro quadrático médio relativo (RRELMSE) definidos por Branco et al. (2012):

$$
\operatorname{RELMB}=\frac{1}{R} \sum_{i=1}^{R} \frac{\left(\hat{\nu}_{i}-\nu\right)}{\nu} \text { e RRELMSE }=\left\{\frac{1}{R} \sum_{i=1}^{R} \frac{\left(\hat{\nu}_{i}-\nu\right)^{2}}{\nu^{2}}\right\}^{1 / 2}
$$

Os resultados são mostrados na Tabela 3.2 e Figura 3.3, em que podemos observar que com a distribuição a priori hierárquica obtemos melhores resultados dos indicadores, quando comparados com as outras propostas. No entanto, é interessante notar que quando mudamos o valor do hiperparâmetro da distribuição a priori exponencial $\nu \sim \operatorname{Exp}\left(\lambda_{i}\right) \mathbb{I}_{\lfloor 2, \infty\rfloor}(\nu)$, os 


\begin{tabular}{|c|c|c|c|c|c|c|c|}
\hline \multirow{3}{*}{ Prioris } & \multicolumn{7}{|c|}{ RELMB } \\
\hline & \multicolumn{7}{|c|}{$\nu$} \\
\hline & 4 & 6 & 8 & 10 & 12 & 14 & 20 \\
\hline $\operatorname{Exp}(0,01)$ & 1,401 & 2,519 & 2,501 & 2,346 & 2,117 & 1,503 & 1,196 \\
\hline $\operatorname{Exp}(0,33)$ & 0,188 & 0,030 & $-0,081$ & $-0,200$ & $-0,301$ & $-0,453$ & $-0,540$ \\
\hline $\operatorname{Exp}(1,50)$ & $-0,176$ & $-0,351$ & $-0,483$ & $-0,564$ & $-0,625$ & $-0,713$ & $-0,761$ \\
\hline $\mathrm{U}(2,100)$ & 1,753 & 3,164 & 3,153 & 2,929 & 2,617 & 1,890 & 1,521 \\
\hline Jeffreys & 0,311 & 0,575 & 0,504 & 0,522 & 0,433 & 0,147 & 0,073 \\
\hline \multirow[t]{2}{*}{ Hierarchical } & 0,274 & 0,420 & 0,501 & 0,393 & 0,257 & $-0,007$ & $-0,043$ \\
\hline & \multicolumn{7}{|c|}{ RRELMSE } \\
\hline \multirow[t]{2}{*}{ Prioris } & \multicolumn{7}{|c|}{$\nu$} \\
\hline & 4 & 6 & 8 & 10 & 12 & 14 & 20 \\
\hline $\operatorname{Exp}(0,01)$ & 2,669 & 3,442 & 3,046 & 2,756 & 2,382 & 1,660 & 1,295 \\
\hline $\operatorname{Exp}(0,33)$ & 0,410 & 0,266 & 0,239 & 0,266 & 0,334 & 0,465 & 0,547 \\
\hline $\operatorname{Exp}(1,50)$ & 0,212 & 0,362 & 0,487 & 0,567 & 0,626 & 0,714 & 0,761 \\
\hline $\mathrm{U}(2,100)$ & 3,246 & 4,221 & 3,740 & 3,344 & 2,872 & 2,030 & 1,604 \\
\hline Jeffreys & 0,796 & 1,126 & 0,954 & 0,935 & 0,784 & 0,488 & 0,407 \\
\hline Hierarchical & 0,607 & 0,852 & 0,897 & 0,718 & 0,548 & 0,395 & 0,326 \\
\hline
\end{tabular}

Tabela 3.2: Viés médio relativo (RELMB) e error quadrático médio relativo (RRELMSE) para diferentes valores de $\nu$ considerando diferentes a priori.

valores dos indicadores RELMB e RRELMSE mudam significativamente, consequentemente as estimativas de $\nu$ são bastante sensíveis à escolha do hiperparâmetro quando consideramos a distribuição a priori exponencial. Apesar das estimativas utilizando a distribuição $a$ priori de Jeffreys terem o mesmo comportamento que as obtidas via distribuição a priori hierárquica, podemos observar contudo que esta última fornece valores menores de RELMB e RRELMSE para os diferentes valores de $\nu$ considerados na análise.

\subsection{Pacote BayesCR}

\subsubsection{Introdução}

O pacote "BayesCR" (Garay \& Lachos, 2013) é uma ferramenta desenvolvida com o objetivo de avaliar os modelos de regressão linear censurados sob a classe de distribuições SMN (SMN-CR) a partir de uma perspectiva Bayesiana. Este pacote obtém as estimativas Bayesianas e realiza uma análise de diagnóstico Bayesiana utilizando as medidas de divergência de Kullback-Leibler.

Este pacote está disponível no repositório CRAN e pode ser instalado livremente a partir de: http://cran.r-project.org/web/packages/BayesCR/index.html. 


\subsubsection{Descrição}

O comando principal do pacote é "Bayes. CR", como é descrito a seguir:

Códigos em $\mathrm{R}$

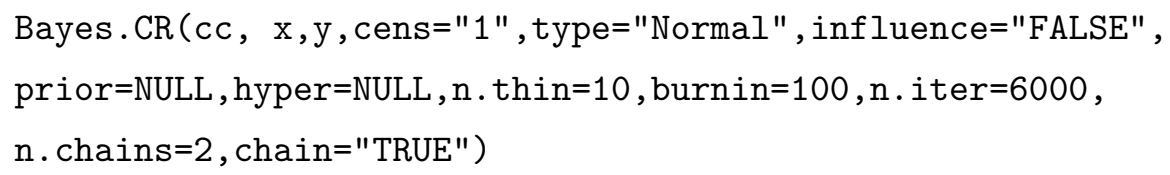

Com os seguintes argumentos:

- $c c \quad$ : vetor de indicadores de censuras. 0 se não for censurado, 1 se for censurado.

- $x \quad$ : vetor de covariáveis.

- $y \quad$ : vetor de respostas.

- cens : tipo de censura. "1" para censura à esquerda e "2" para censura à direita.

- type : distribuições a serem consideradas na estimação ("Normal", "T", "PearsonVII", "Slash" e "NormalC").

- influence : se for TRUE, será construído os gráficos das medidas de divergência.

- prior : distibuição a priori para o parâmetro $\nu$ no caso t de Student ( "Exp" para a distribuição a priori Exponencial, "Jeffreys" para a priori objetiva de Jeffreys, "Unif" para a distribuição a priori Uniforme e "Hierar" para a priori hierárquica.

- hyper : valor do hiperparâmetro para a distribuição a priori Exponencial.

- n.thin : tamanho do espaçamento a considerar.

- burnin : quantidade de iterações iniciais das cadeias a serem descartadas.

- n.iter : número de iterações consideradas em cada cadeia para a análise.

- n.chains : número de cadeias considerados para a análise.

- chain : se for TRUE, todas as cadeias (depois do burn-in) serão recuperadas.

\subsubsection{Utilização do pacote}

Com o intuito de exemplificar a utilização do pacote BayesCR, definiremos a seguinte sequência de passos: 
Passo 1. Leitura dos dados, por exemplo suponha que queremos encontrar as estimativas Bayesianas do conjunto de dados Salarios, apresentados na Seção 2.10.

Códigos em $R$

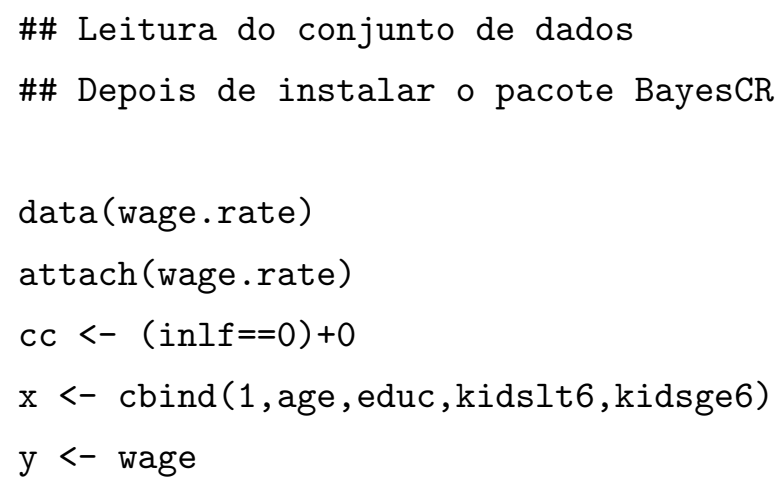

Passo 2. Estimação, para encontrar as estimativas Bayesianas do conjunto de dados do Passo 1, considerando o modelo censurado t de Student (T-CR), com distribuição a priori hierárquica para $\nu$, faz-se.

\section{Códigos em $\mathrm{R}$}

T<- Bayes.CR (cc, x, y, cens="1", type="T", influence="FALSE", prior="Hierar", hyper=NULL, n. thin=10, burnin=500,n.iter=8000, n. chains $=2$, chain="TRUE")

Passo 3. Resultados obtidos com o pacote BayesCR

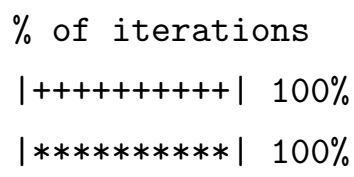

Posterior mean(Mean), standard deviation(Sd) and HPD interval

$\begin{array}{lrrrrr} & \text { Mean } & \text { Sd } & \text { HPD }(95 \%) & \text { Rhat } \\ & -1,24700 & 1,37630 & -3,73629 & 1,60685 & 1,00093 \\ \text { age } & -0,10980 & 0,02207 & -0,15055 & -0,06628 & 0,99976 \\ \text { educ } & 0,65500 & 0,07312 & 0,51827 & 0,79652 & 1,00677 \\ \text { kidslt6 } & -3,15381 & 0,39011 & -3,91328 & -2,40463 & 1,00249 \\ \text { kidsge6 } & -0,29223 & 0,13072 & -0,56352 & -0,04986 & 1,00044\end{array}$




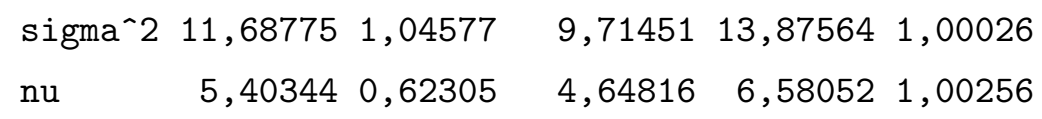

\subsection{Aplicação: Salários}

Prosseguimos com a análise Bayesiana dos dados descritos na Seção 2.10, utilizando o pacote BayesCR.

\subsubsection{Estimação}

Para encontrar as estimativas Bayesianas, consideramos a distribuições a priori discutidas na Subseção 3.2.1. No caso do $\nu$ para o modelo T-CR, assumimos a priori hierárquica. Com esta estrutura de distribuições a prioris, foram geradas quatro cadeias MCMC independentes, de tamanho 50.000, descartando as primeiras 10.000 iterações (burn-in) para eliminar o efeito dos valores iniciais. Por outro lado, visando minimizar o problema de autocorrelação das cadeias geradas, consideramos um espaçamento de comprimento 20 (thin). Para monitorar a convergência das cadeias, utilizamos o gráfico do histórico das cadeias, o gráfico de autocorrelação e as estimativas dos fatores de redução de escala potencial $\widehat{R}$ (Gelman \& Rubin., 1992).

Na Tabela 3.3, apresentamos o resumo das distribuições a posteriori (a média a posteriori (Média), o desvio padrão (DP) e o intervalo HPD de 95\%) para os diferentes parâmetros dos diferentes modelos SMN-CR. Podemos notar que as estimativas a posteriori são similares para os quatro modelos estimados, no entanto, o desvio padrão dos modelos T-CR, SL-CR e CN-CR são menores que os obtidos com o modelo N-CR, indicando que os modelos com caudas mais pesadas que a normal produzem estimativas Bayesianas mais precisas. Para os modelos T-CR e N-CR, o valor estimado de $\nu$ é pequeno, indicando que a suposição de normalidade não é apropriada para o conjunto de dados.

Também é possível ver que o intervalo HPD de (95\%) para o intercepto $\beta_{1}$, contêm o valor zero, isto é, o intercepto é considerado não significante para os quatro modelos ao nível de $5 \%$. Os parâmetros $\beta_{2}, \beta_{3}, \beta_{4}$ e $\beta_{5}$ são significantes para os modelos T-CR, SL-CR e CN-CR. No caso do modelo N-CR, o parâmetro $\beta_{5}$ (o número de crianças com idade entre seis e dezenove anos) não é significativo, já que o intervalo HPD inclui o valor zero. Como esperado, os valores das médias na Tabela 3.3 estão bem próximos das estimativas 


\begin{tabular}{|c|c|c|c|c|c|c|c|c|}
\hline \multirow{3}{*}{ Parâmetros } & \multicolumn{8}{|c|}{ Modelos } \\
\hline & \multicolumn{4}{|c|}{ N-CR } & \multicolumn{4}{|c|}{ T-CR } \\
\hline & Média & DP & HPD $(95 \%)$ & & Média & DP & HPD $(95 \%)$ & \\
\hline$\beta_{1}$ & $-2,7695$ & 1,7542 & $(-6,247 ; 0,579)$ & & $-1,1945$ & 1,4236 & $(-4,100 ; 1,469)$ & \\
\hline$\beta_{2}$ & $-0,1056$ & 0,0278 & $(-0,157 ;-0,049)$ & $*$ & $-0,110$ & 0,0229 & $(-0,154 ;-0,066)$ & * \\
\hline$\beta_{3}$ & 0,7324 & 0,0847 & $(0,570 ; 0,899)$ & * & 0,6534 & 0,0719 & $(0,516 ; 0,801)$ & * \\
\hline$\beta_{4}$ & $-3,0521$ & 0,4513 & $(-3,970 ;-2,210)$ & $*$ & $-3,1649$ & 0,3885 & $(-3,914 ;-2,392)$ & * \\
\hline$\beta_{5}$ & $-0,2197$ & 0,1557 & $(-0,519 ; 0,082)$ & & $-0,2905$ & 0,1310 & $(-0,546 ;-0,034)$ & * \\
\hline$\sigma^{2}$ & 21,3451 & 1,6156 & $(18,110 ; 24,426)$ & $*$ & 11,5843 & 1,0460 & $(9,539 ; 13,625)$ & * \\
\hline$\nu$ & - & - & - & & 5,2789 & 0,6709 & $(4,543 ; 6,581)$ & * \\
\hline \multirow[t]{2}{*}{ Parâmetros } & \multicolumn{4}{|c|}{ SL-CR } & \multicolumn{4}{|c|}{ CN-CR } \\
\hline & Média & DP & HPD $(95 \%)$ & & Média & DP & HPD (95\%) & \\
\hline$\beta_{1}$ & $-1,1931$ & 1,4000 & $(-3,909 ; 1,613)$ & & $-1,6361$ & 1,4675 & $(-4,388 ; 1,276)$ & \\
\hline$\beta_{2}$ & $-0,1093$ & 0,0223 & $(-0,151 ;-0,063)$ & $*$ & $-0,1057$ & 0,0233 & $(-0,150 ;-0,059)$ & * \\
\hline$\beta_{3}$ & 0,6494 & 0,0710 & $(0,518 ; 0,791)$ & * & 0,6651 & 0,0722 & $(0,518 ; 0,797)$ & * \\
\hline$\beta_{4}$ & $-3,1325$ & 0,3905 & $(-3,912 ;-2,390)$ & $*$ & $-3,0721$ & 0,3949 & $(-3,866 ;-2,295)$ & * \\
\hline$\beta_{5}$ & $-0,2959$ & 0,1272 & $(-0,532 ;-0,036)$ & $*$ & $-0,2796$ & 0,1323 & $(-0,517 ;-0,009)$ & * \\
\hline$\sigma^{2}$ & 6,9515 & 0,8672 & $(5,374 ; 8,723)$ & $*$ & 12,7946 & 1,9148 & $(9,797 ; 16,931)$ & * \\
\hline$\nu$ & 1,4379 & 0,2094 & $(1,059 ; 1,848)$ & $*$ & 0,0989 & 0,0568 & $(0,015 ; 0,205)$ & * \\
\hline$\gamma$ & - & - & - & & 0,1761 & 0,0892 & $(0,113 ; 0,237)$ & * \\
\hline
\end{tabular}

Tabela 3.3: Média a posteriori, desvio padrão (DP) e intervalo HPD de $95 \%$ para os parâmetros dos modelos SMN-CR.

de máxima verossimilhança, obtidas com o algoritmo EM e apresentadas na Tabela 2.2.

\begin{tabular}{llllll}
\hline Modelo & LPML & DIC & EAIC & EBIC & $p_{B}$ \\
\hline N-CR & $-1489,68$ & 8901,99 & 2967,38 & 2976,63 & 0,609 \\
T-CR & $-1447,26$ & 8656,71 & 2887,62 & 2901,49 & 0,332 \\
SL-CR & $-1443,63$ & 8631,85 & 2878,64 & 2892,51 & 0,433 \\
CN-CR & $-1475,16$ & 8655,73 & 2878,74 & 2892,61 & 0,762 \\
\hline
\end{tabular}

Tabela 3.4: Critérios de seleção de modelos.

A Tabela 3.4 apresenta uma comparação entre os quatro diferentes modelos SMN-CR utilizando os critérios de comparação de modelos, descritos na Seção 3.3. Através desta tabela, pode-se observar que os modelos T-CR, SL-CR e CN-CR apresentam um desempenho significativamente melhor do que o modelo N-CR. O p-valor Bayesiano $p_{B}$ reportado nesta tabela, indica que não há problemas de ajuste para os quatro modelos SMN-CR considerados.

A Figura 3.4 apresenta o histórico das cadeias e as densidades marginais a posteriori aproximadas para os parâmetros $\beta_{2}$ e $\nu$, considerando o modelo SL-CR. 

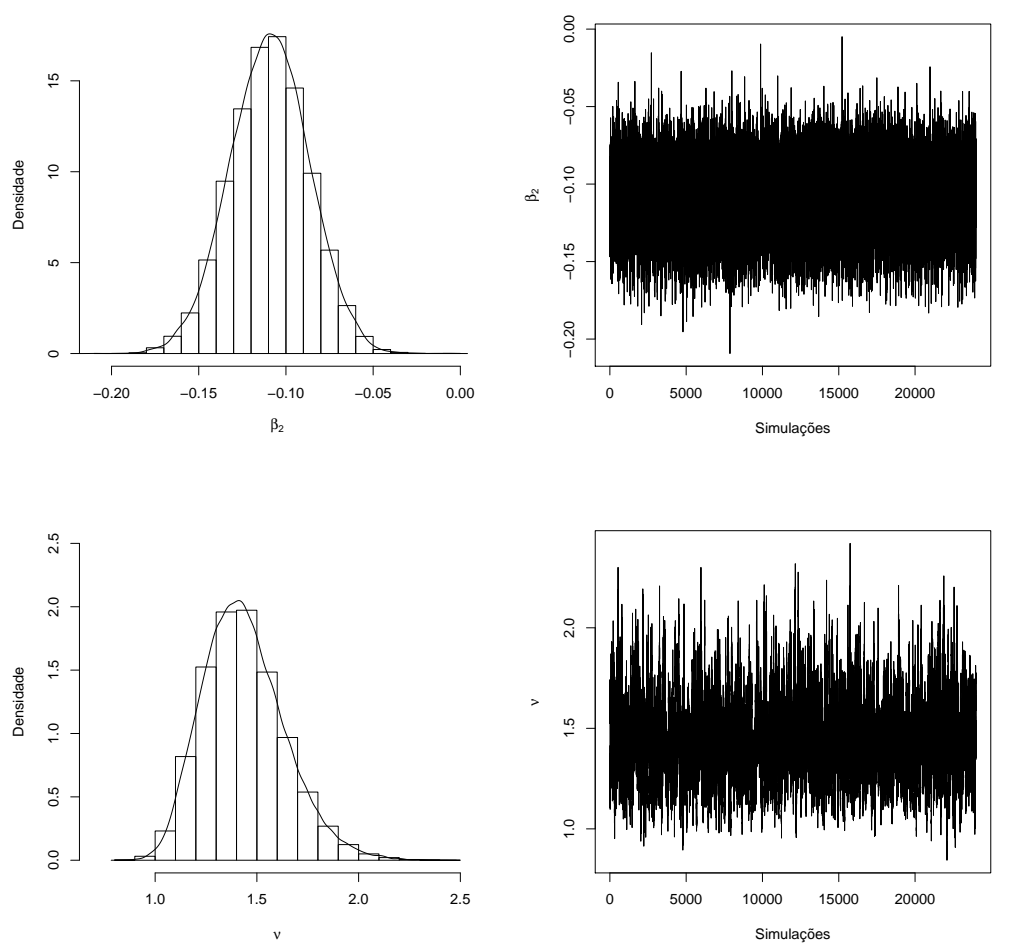

Figura 3.4: Histórico das cadeias e densidades marginais a posteriori aproximadas, considerando o modelo SL-CR

\subsubsection{Diagnóstico de influência Bayesiana}

Considerando a amostra das distribuições a posteriori dos parâmetros dos modelos SMN-CR, calculamos as medidas q-divergência, descritas na Seção 3.4 (distâncias $K L, J$ e $\left.L_{1}\right)$. Note que para o modelo N-CR, as observações $\sharp 74, \sharp 185, \sharp 349, \sharp 394$ e $\sharp 408$ são consideradas como influentes. No entanto para os modelos T-CR e SL-CR não foram detectadas observações influentes (veja Figura 3.5).

Nos Capítulos 2 e 3 foram abordados os modelos lineares censurados, sob a classe de distribuições SMN, denotados por SMN-CR, obtendo resultados interessantes que podem ser utilizados nas diferentes áreas da pesquisa. Entretanto, estes resultados serão necessários para uma extensão natural, que são os modelos não lineares censurados sob a classe de distribuições SMN, que será o objetivo do próximo capítulo. 
(a)

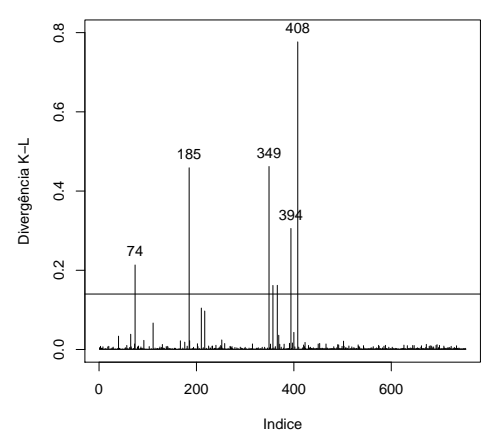

(a)

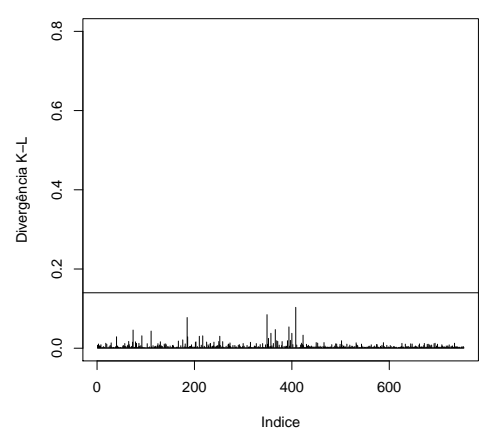

(a)

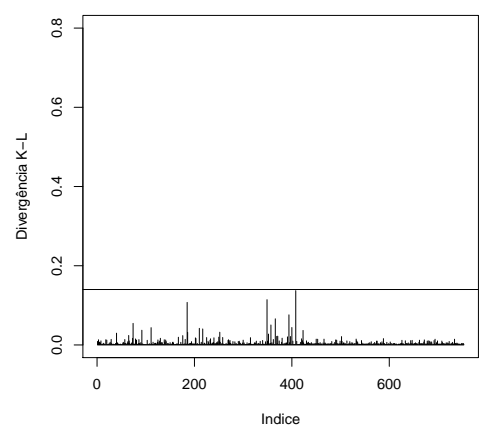

(b)

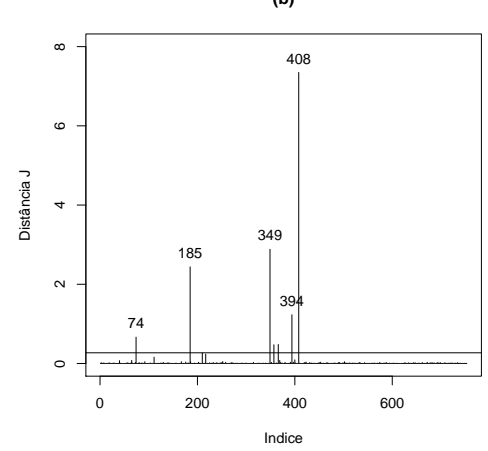

(b)

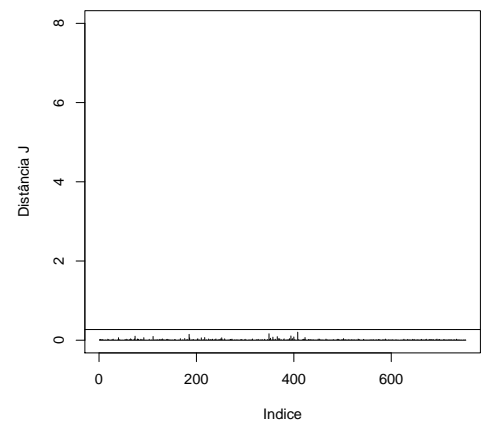

(b)

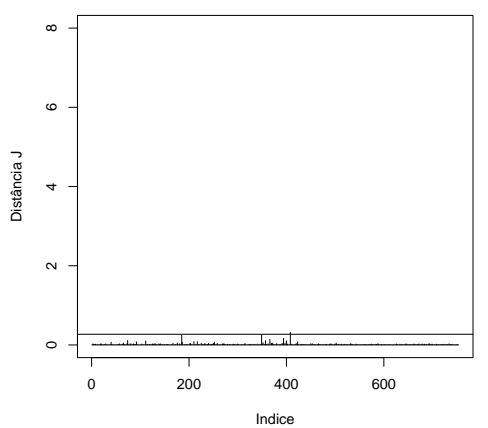

(c)

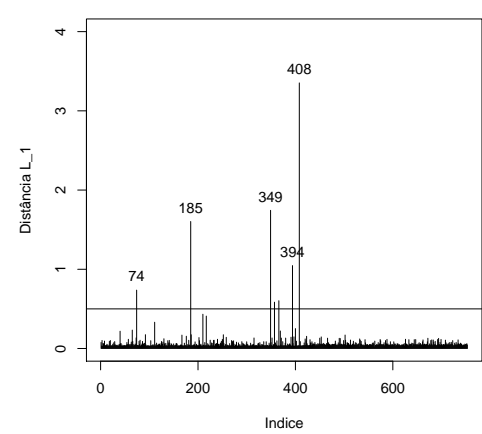

(c)

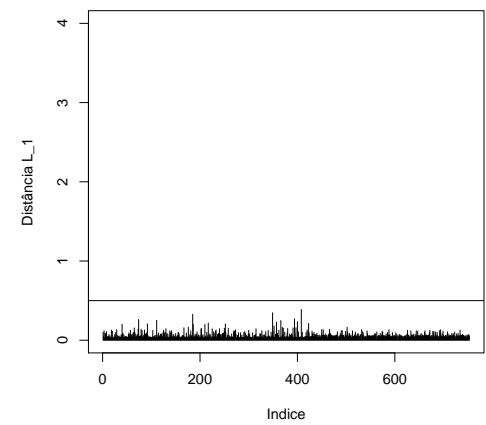

(c)

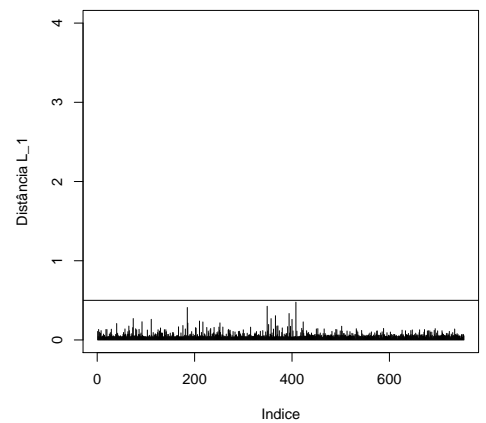

Figura 3.5: Medidas de diagnóstico de influência para os modelos N-CR (1ra Linha), T-CR (2da Linha) e SL-CR (3ra Linha): (a) K-L divergência, (b) distância J, (c) distância $L_{1}$ 


\section{Capítulo 4}

\section{Modelos de regressão não linear censurados sob a classe SMN}

\subsection{Introdução}

Os modelos normais de regressão não linear (NL) podem ser considerados como uma das ferramentas mais populares para a análise estatística de dados. No entanto, como no caso dos modelos lineares, os fenômenos sob investigação nem sempre estão de acordo com a suposição rotineira de normalidade, seja pela falta de simetria ou pela presença de observações atípicas. Para lidar com este problema, como no caso dos modelos lineares, algumas propostas tem sido discutidas na literatura, substituindo a suposição de normalidade por uma classe de distribuições mais flexíveis. Dentro deste contexto, e sendo o foco deste Capítulo os modelos não lineares, podemos citar os seguintes trabalhos: Cysneiros \& Vanegas (2008), que fizeram um estudo das propriedades estatísticas dos resíduos nos modelos de regressão NL simétricos. Vanegas \& Cysneiros (2010) que propuseram um procedimento de diagnóstico baseado no método de deleção de casos para os modelos de regressão NL simétricos. Por outro lado, Cancho et al. (2009) introduziram os modelos de regressão não linear Skew Normal (SN-NL) e apresentaram uma análise inferencial completa, incluindo o algoritmo EM para encontrar as estimativas de MV.

No contexto das censuras, os modelos não lineares censurados normais, denotados por NLCR, estão presentes em muitas áreas da pesquisa e tem sido estudados nos últimos anos, como por exemplo Heuchenne \& Keilegom (2007), que apresentam um estudo clássico dos modelos NLCR, desenvolvendo uma extensão dos procedimentos de mínimos quadrados para estimar os parâmetros. No entanto, sobre o melhor de nosso conhecimento, ainda não foram estudados os modelos não lineares censurado sob uma classe de distribuições mais flexíveis do que a distribuição normal, como por exemplo a classe de distribuições SMN apresentada no Capítulo 2.

Assim, neste Capítulo apresentaremos uma extensão dos modelos NLCR, denotados por SMN-NLCR, assumindo que os erros tem uma distribuição na classe SMN. Desenvolveremos um interessante algoritmo tipo EM para encontrar as estimativas de MV dos parâmetros, 
utilizando os resultados obtidos nos Capítulos 1 e 2. Avaliaremos a performance das estimativas de MV através de estudos de simulação e ilustraremos as técnicas desenvolvidas neste Capítulo aplicando-as a um conjunto de dados reais.

\subsection{Modelo de regressão não linear censurado SMN}

\subsubsection{Descrição do modelo}

Considere o modelo de regressão não linear sob a classe de distribuições SMN, que pode ser escrito como:

$$
Y_{i}=\eta\left(\mathbf{x}_{i}, \boldsymbol{\beta}\right)+\varepsilon_{i}, \text { em que } \varepsilon_{i} \stackrel{\text { iid }}{\sim} \operatorname{SMN}\left(0, \sigma^{2}, \boldsymbol{\nu}\right), \quad i=1, \ldots, n
$$

em que $Y_{i}$ são as respostas, $\eta_{i}(\boldsymbol{\beta})=\eta\left(\mathbf{x}_{i}, \boldsymbol{\beta}\right)$ é uma função injetiva e duas vezes diferenciáveis sob o vetor de parâmetros $\boldsymbol{\beta}=\left(\beta_{1}, \ldots, \beta_{p}\right)^{\top}, \mathbf{D}_{i \beta}=\partial \eta_{i}(\boldsymbol{\beta}) / \partial \boldsymbol{\beta}$ é a matriz de derivadas de posto $p(p<n)$ e $\mathbf{x}_{i}$ é o vetor de variáveis explicativas para a $i$-ésima observação.

Assim, de (4.1) temos que $Y_{i} \stackrel{\text { ind }}{\sim} \operatorname{SMN}\left(\eta\left(\mathbf{x}_{i}, \boldsymbol{\beta}\right), \sigma^{2}, \boldsymbol{\nu}\right)$, para $i=1, \ldots, n$, que é definido como modelo de regressão não linear SMN e é denotado por SMN-NLR.

Estamos interessados no caso em que as observações com censura intervalar podem ocorrer, para isto seguimos a proposta dada em Vaida \& Liu (2009) e Matos et al. (2013), no qual a resposta observada $Y$ não é completa para todas as observações. Assim, seja $\left(\mathbf{V}_{i}, C_{i}\right)$ os dados observados para o $i$-ésimo caso, em que $\mathbf{V}_{i}$ representa o valor não censurado $\mathbf{V}_{i}=V_{0 i}$ ou a censura intervalar $\mathbf{V}_{i}=\left(V_{1 i}, V_{2 i}\right)$ e $C_{i}$ é o indicador de censura temos que:

$$
V_{1 i} \leq Y_{i} \leq V_{2 i} \quad \text { se } C_{i}=1, \text { e } Y_{i}=V_{0 i} \text { se } C_{i}=0
$$

Portanto, considerando (4.1) e (4.2), definimos os modelos não lineares censurados sob a classe de distribuições SMN, os quais denotaremos por SMN-CNLR.

\subsubsection{Função de log-verosimilhança}

Seja $\boldsymbol{\theta}=\left(\beta^{\top}, \sigma^{2}, \boldsymbol{\nu}\right)^{\top}$ o vetor dos parâmetros do modelo SMN-CNLR e $\mathbf{y}_{\text {obs }}$ uma amostra observada com $\mathbf{V}=\left(\mathbf{V}_{1}^{\top}, \ldots, \mathbf{V}_{n}^{\top}\right)^{\top}$ e $\mathbf{C}=\left(C_{1}, \ldots, C_{n}\right)^{\top}$. Como visto na Seção 2.3, supondo que há $m$ possíveis valores censurados da variável de interesse, podemos particionar a amostra observada $\mathbf{y}_{\text {obs }}$ em duas subamostras de $m$ valores censurados e $n-m$ valores não censurados, assim $\mathbf{y}_{\mathrm{obs}}=\left(\mathbf{C}^{\top}, \mathbf{V}^{\top}\right)=\left\{\mathbf{V}_{1}, \ldots, \mathbf{V}_{m}, y_{m+1}, \ldots, y_{n}\right\}$.

Então a função de log-verossimilhança, dada a amostra observada $\mathbf{y}_{\text {obs }}$, é dada por

$$
\begin{aligned}
\ell\left(\boldsymbol{\theta} \mid \mathbf{y}_{\text {obs }}\right)= & \sum_{i=1}^{m} \log \left[F_{S M N}\left(\frac{V_{2 i}-\eta\left(\mathbf{x}_{i}, \boldsymbol{\beta}\right)}{\sigma}\right)-F_{S M N}\left(\frac{V_{1 i}-\eta\left(\mathbf{x}_{i}, \boldsymbol{\beta}\right)}{\sigma}\right)\right] \\
& +\sum_{i=m+1}^{n} \log \left[f_{S M N}\left(y_{i} \mid \eta\left(\mathbf{x}_{i}, \boldsymbol{\beta}\right), \sigma^{2}, \boldsymbol{\nu}\right)\right] .
\end{aligned}
$$

Para estimar os parâmetros do modelo SMN-CNLR, utilizaremos o algoritmo ECME desenvolvido na Seção 2.4 para o caso dos modelos lineares SMN-CR. 


\subsection{Estimação dos parâmetros}

\subsubsection{O algoritmo ECME para os modelos SMN-NLCR}

Com o intuito de estimar os parâmetros dos modelos SMN-CNLR, utilizaremos como base o algoritmo tipo EM, que foi desenvolvido na Seção 2.4. Assim, pela Definição 1.1, o modelo SMN-CNLR tem a seguinte representação hierárquica:

$$
Y_{i} \mid U_{i}=u_{i} \sim \mathrm{N}\left(\eta\left(\mathbf{x}_{i}, \boldsymbol{\beta}\right), u_{i}^{-1} \sigma^{2}\right) ; \quad U_{i} \sim H(\cdot \mid \boldsymbol{\nu}) .
$$

Se a $i$-ésima observação for censurada, podemos considerar $y_{i}$ como a realização de uma variável latente não observada $Y_{i} \sim \operatorname{SMN}\left(\eta\left(\mathbf{x}_{i}, \boldsymbol{\beta}\right), \sigma^{2}, \boldsymbol{\nu}\right), i=1, \ldots, m$. Assim, como na Seção 2.4, considerando os dados completos $\mathbf{z}=\left\{\mathbf{y}_{\text {obs }}, y_{1}, \ldots, y_{m}, u_{1}, \ldots, u_{n}\right\}=$ $\left\{y_{1}, \ldots, y_{n}, u_{1}, \ldots, u_{n}\right\}$ e a equação (4.4), temos que a função de log-verossimilhança completa para $\boldsymbol{\theta}$, associada com $\mathbf{z}$, é dada por:

$\ell_{c}(\boldsymbol{\theta} \mid \mathbf{z})=\operatorname{cte}-\frac{n}{2} \log \left(\sigma^{2}\right)+\frac{1}{2} \sum_{i=1}^{n} \log \left(u_{i}\right)-\frac{1}{2 \sigma^{2}} \sum_{i=1}^{n} u_{i}\left(y_{i}-\eta\left(\mathbf{x}_{i}, \boldsymbol{\beta}\right)\right)^{2}+\sum_{i=1}^{n} \log \left(h\left(u_{i} \mid \boldsymbol{\nu}\right)\right)$

Na $k$-ésima iteração de nosso algoritmo, calcularemos a função $Q$, definida como

$$
Q\left(\boldsymbol{\theta} \mid \boldsymbol{\theta}^{(k)}\right)=\mathrm{E}_{\boldsymbol{\theta}^{(k)}}\left[\ell_{c}(\boldsymbol{\theta} \mid \mathbf{Z}) \mid \mathbf{y}_{\text {obs }}\right],
$$

a qual, pode ser escrita de forma reduzida por

$$
\begin{aligned}
Q\left(\boldsymbol{\theta} \mid \boldsymbol{\theta}^{(k)}\right) & =-\frac{n}{2} \log \left(\sigma^{2}\right)-\frac{1}{2 \sigma^{2}} \sum_{i=1}^{n}\left[\mathcal{E}_{2 i}\left(\boldsymbol{\theta}^{(k)}\right)-2 \mathcal{E}_{1 i}\left(\boldsymbol{\theta}^{(k)}\right) \eta\left(\mathbf{x}_{i}, \boldsymbol{\beta}\right)+\mathcal{E}_{0 i}\left(\boldsymbol{\theta}^{(k)}\right) \eta\left(\mathbf{x}_{i}, \boldsymbol{\beta}\right)^{2}\right] \\
& +\frac{1}{2} \sum_{i=1}^{n} \mathrm{E}_{\boldsymbol{\theta}^{(k)}}\left[\log \left(U_{i}\right) \mid \mathbf{V}_{i}, C_{i}\right]+\sum_{i=1}^{n} \mathrm{E}_{\boldsymbol{\theta}^{(k)}}\left[\log \left(h\left(U_{i} \mid \boldsymbol{\nu}\right)\right) \mid \mathbf{V}_{i}, C_{i}\right]
\end{aligned}
$$

em que $\mathcal{E}_{s i}\left(\boldsymbol{\theta}^{(k)}\right)=\mathrm{E}_{\boldsymbol{\theta}^{(k)}}\left[U_{i} Y_{i}^{s} \mid y_{\mathrm{obs}_{i}}\right]$ para $s=0,1,2$, podem ser calculadas utilizando a Proposição 1 ou os resultados obtidos em Osorio et al. (2007).

Em resumo, o procedimento do algoritmo ECME para encontrar as estimativas de MV dos parâmetros dos modelos SMN-NLCR, é dado por:

\section{Passo E:}

Dado $\boldsymbol{\theta}=\boldsymbol{\theta}^{(k)}$, para $i=1, \ldots, n$, temos que

- Se a $i$-ésima observação for não censurada, então, para $s=0,1,2$, calculamos $\mathcal{E}_{s i}\left(\boldsymbol{\theta}^{(k)}\right)$, dado em (2.8);

- Se a $i$-ésima observação for censurada, então, para $s=0,1,2$, obtemos $\mathcal{E}_{s i}\left(\boldsymbol{\theta}^{(k)}\right)$, dado em (2.9).

\section{Passo CM:}

Atualizamos $\boldsymbol{\theta}^{(k)}$ pela maximização $Q\left(\boldsymbol{\theta} \mid \boldsymbol{\theta}^{(k)}\right)$ sobre $\boldsymbol{\theta}$, o que leva as seguintes expressões:

$$
\begin{aligned}
\widehat{\boldsymbol{\beta}}^{(k+1)} & =\operatorname{argmin}_{\boldsymbol{\beta}}\left(\boldsymbol{\tau}^{(k)}-\boldsymbol{\eta}(\boldsymbol{\beta}, \mathbf{x})\right)^{\top} \widehat{\mathbf{U}}^{(k)}\left(\boldsymbol{\tau}^{(k)}-\boldsymbol{\eta}(\boldsymbol{\beta}, \mathbf{x})\right) \\
\sigma^{2(k+1)} & =\frac{1}{n} \sum_{i=1}^{n}\left[\mathcal{E}_{2 i}\left(\boldsymbol{\theta}^{(k)}\right)-2 \mathcal{E}_{1 i}\left(\boldsymbol{\theta}^{(k)}\right) \eta\left(\mathbf{x}_{i}, \boldsymbol{\beta}^{(k+1)}\right)+\mathcal{E}_{0 i}\left(\boldsymbol{\theta}^{(k)}\right)\left(\eta\left(\mathbf{x}_{i}, \boldsymbol{\beta}^{(k+1)}\right)\right)^{2}\right] .(4 .
\end{aligned}
$$




\section{Passo CML:}

Atualizamos $\nu^{(k)}$ pela maximização da função de verossimilhança marginal, o que leva a seguinte expressão

$$
\begin{aligned}
\boldsymbol{\nu}^{(k+1)}= & \operatorname{argmax}_{\nu}\left\{\sum_{i=1}^{m} \log \left[F_{S M N}\left(\frac{V_{2 i}-\eta\left(\mathbf{x}_{i}, \boldsymbol{\beta}^{(k+1)}\right)}{\sigma^{(k+1)}}\right)-F_{S M N}\left(\frac{V_{1 i}-\eta\left(\mathbf{x}_{i}, \boldsymbol{\beta}^{(k+1)}\right)}{\sigma^{(k+1)}}\right)\right]\right. \\
& \left.+\sum_{i=m+1}^{n} \log \left[f_{S M N}\left(y_{i} \mid \eta\left(\mathbf{x}_{i}, \boldsymbol{\beta}^{(k+1)}\right), \sigma^{2(k+1)}, \boldsymbol{\nu}\right)\right]\right\}
\end{aligned}
$$

em que $\boldsymbol{\eta}(\boldsymbol{\beta}, \mathbf{x})=\left(\eta\left(\boldsymbol{\beta}, \mathbf{x}_{1}\right), \ldots, \eta\left(\boldsymbol{\beta}, \mathbf{x}_{n}\right)\right)^{\top}, \widehat{\mathbf{U}}^{(k)}=\operatorname{Diag}\left(\mathcal{E}_{01}\left(\boldsymbol{\theta}^{(k)}\right), \ldots, \mathcal{E}_{0 n}\left(\boldsymbol{\theta}^{(k)}\right)\right)$ e $\widehat{\boldsymbol{\tau}}^{(k)}=$ $\left({\widehat{\tau_{1}}}^{(k)}, \ldots,{\widehat{\tau_{n}}}^{(k)}\right)^{\top}$ representa a resposta observada corrigida com $\widehat{\tau}_{i}^{(k)}=\mathcal{E}_{1 n}\left(\boldsymbol{\theta}^{(k)}\right) / \mathcal{E}_{0 n}\left(\boldsymbol{\theta}^{(k)}\right)$.

Dado um conjunto de valores iniciais apropriados $\widehat{\boldsymbol{\theta}}^{(0)}$, como descritos na seguinte Subseção, o procedimento ECME é iterado até que um critério de convergência, como por exemplo ||$\ell\left(\boldsymbol{\theta}^{(k+1)} \mid \mathbf{y}_{\text {obs }}\right)-\ell\left(\boldsymbol{\theta}^{(k)} \mid \mathbf{y}_{\text {obs }}\right)||$ ou ||$\ell\left(\boldsymbol{\theta}^{(k+1)} \mid \mathbf{y}_{\text {obs }}\right) / \ell\left(\boldsymbol{\theta}^{(k)} \mid \mathbf{y}_{\text {obs }}\right)-1||$, seja suficientemente pequena.

Como na Seção 2.4, adotamos esta estratégia para atualizar a estimativa de $\boldsymbol{\nu}$ pela maximização direta da log-verossimilhança marginal, com o intuito de contornar o cálculo de $\mathrm{E}_{\boldsymbol{\theta}^{(k)}}\left[\log \left(U_{i}\right) \mid y_{\mathrm{obs}_{i}}\right]$ e $\mathrm{E}_{\boldsymbol{\theta}^{(k)}}\left[\log \left(h\left(U_{i} \mid \boldsymbol{\nu}\right)\right) \mid y_{\mathrm{obs}_{i}}\right]$.

\subsubsection{Especificação dos valores iniciais}

É conhecido que as estimativas de MV dos modelos não lineares podem apresentar alguns problemas computacionais, já que o método pode não obter o máximo global, quando os valores iniciais estão longe dos valores reais dos parâmetros. Assim, a escolha dos valores iniciais para o algoritmo EM no contexto não linear têm uma grande influência na estimação dos parâmetros.

Para nosso modelo SMN-NLCR, consideramos o seguinte procedimento:

- Calcular as estimativas de MV $\widehat{\boldsymbol{\beta}}^{(0)}$ e $\widehat{\sigma}^{2(0)}$, utilizando o método padrão não linear de mínimos quadrados, denotado por NLLS, o qual pode ser calculado considerando a função do R nls (). Esses valores devem ser calculados considerando dados completos, isto é, assumindo que a censura não está presente nos dados.

- Utilizamos as estimativas dos parâmetros de regressão e de escala, obtidas no item anterior, como os valores iniciais para o parâmetros correspondentes para os modelos N-NLCR, T-NLCR, S-NLCR e CN-NLCR;

- Com o intuito de estimar o parâmetro de mistura $\nu$ sob os diferentes modelos, assumimos $\nu=3$ para os modelos ST-NLCR e SL-NLCR e $\boldsymbol{\nu}=(0.1,0.1)$ para o modelo CN-NLCR como valores iniciais, respectivamente 


\subsection{Erro padrão aproximado para os efeitos fixos}

Com o objetivo de avaliar a variabilidade do estimador de MV de $\boldsymbol{\theta}, \widehat{\boldsymbol{\theta}}=\left(\widehat{\boldsymbol{\beta}}, \widehat{\sigma^{2}}\right)^{\top}$, utilizaremos a metodologia desenvolvida na Seção 4.4, utilizando a fórmula dada em Louis (1982).

Seja $\ell_{c}\left(\boldsymbol{\theta} \mid \mathbf{z}_{i}\right)$ a função de log-verossimilhança obtida a partir da $i$-ésima observação completa $\mathbf{z}_{i}=\left(y_{\mathrm{obs}_{i}}, y_{i}, u_{i}\right)^{\top}$. A estimação da variância de $\widehat{\boldsymbol{\beta}}$, denotada por $\operatorname{Var}_{\boldsymbol{\theta}}[\widehat{\boldsymbol{\beta}}]$, é dada pela inversa da matriz

$$
-\sum_{i=1}^{n}\left\{\mathrm{E}_{\boldsymbol{\theta}}\left[\frac{\partial^{2} \ell\left(\boldsymbol{\theta} \mid y_{o b s_{i}}\right)}{\partial \boldsymbol{\beta} \partial \boldsymbol{\beta}^{T}}\right]-\operatorname{Var}_{\boldsymbol{\theta}}\left[\frac{\partial \ell_{c}\left(\boldsymbol{\theta} \mid \mathbf{Z}_{i}\right)}{\partial \boldsymbol{\beta}} \mid y_{o b s_{i}}\right]\right\},
$$

avaliada em $\widehat{\boldsymbol{\theta}}$.

Sob o modelo SMN-NLR (4.1), isto é o modelo não censurado, a Matriz de Informação correspondente a $\boldsymbol{\beta}$ é bloco diagonal e assume a seguinte forma

$$
-\sum_{i=1}^{n} \mathrm{E}\left[\frac{\partial^{2} \ell\left(\boldsymbol{\theta} \mid y_{o b s_{i}}\right)}{\partial \boldsymbol{\beta} \partial \boldsymbol{\beta}^{T}}\right]=\frac{4}{\sigma^{2}} \sum_{i=1}^{n}\left(\mathbf{D}_{i \beta} \mathbf{D}_{i \beta}^{\top}\right) d_{g i},
$$

em que $d_{g i}=\mathrm{E}\left[Z_{i}^{2} W_{g}^{2}\left(Z_{i}^{2}\right)\right], Z_{i} \sim \operatorname{SMN}(0,1, \nu)$ e $W_{g}(z)=g^{\prime}(z) / g(z)$, em que $g^{\prime}(z)$ é a primeira derivada de $g(\cdot)$ em relação a $z$. Para maiores detalhes, veja Lange et al. (1989) e Osorio et al. (2007, Sec 2).

A Matriz de Informação ajustada, para as observações censuradas, é dada por

$$
\begin{aligned}
& \sum_{i=1}^{m} \operatorname{Var}_{\boldsymbol{\theta}}\left[\frac{\partial \ell_{c}\left(\boldsymbol{\theta} \mid \mathbf{Z}_{i}\right)}{\partial \boldsymbol{\beta}} \mid V_{1 i} \leq Y_{i} \leq V_{2 i}\right] \\
= & \sum_{i=1}^{m} \operatorname{Var}_{\boldsymbol{\theta}}\left[\frac{1}{\sigma^{2}} \mathbf{D}_{i \beta}\left(U_{i} Y_{i}-U_{i} \eta\left(\mathbf{x}_{i}, \boldsymbol{\beta}\right)\right) \mid V_{1 i} \leq Y_{i} \leq V_{2 i}\right] \\
= & \frac{1}{\sigma^{4}} \sum_{i=1}^{m}\left(\mathbf{D}_{i \beta} \mathbf{D}_{i \beta}^{\top}\right) \operatorname{Var}_{\boldsymbol{\theta}}\left[U_{i} Y_{i}-U_{i} \eta\left(\mathbf{x}_{i}, \boldsymbol{\beta}\right) \mid V_{1 i} \leq Y_{i} \leq V_{2 i}\right] \\
= & \frac{1}{\sigma^{4}} \sum_{i=1}^{m}\left(\mathbf{D}_{i \beta} \mathbf{D}_{i \beta}^{\top}\right)\left\{\operatorname{Var}_{\boldsymbol{\theta}}\left[U_{i} Y_{i}^{\top} \mid V_{1 i} \leq Y_{i} \leq V_{2 i}\right]\right. \\
& +\operatorname{Var}_{\boldsymbol{\theta}}\left[U_{i} \eta\left(\mathbf{x}_{i}, \boldsymbol{\beta}\right) \mid V_{1 i} \leq Y_{i} \leq V_{2 i}\right] \\
& \left.-2 \operatorname{Cov}_{\boldsymbol{\theta}}\left[U_{i} Y_{i}, U_{i} Y_{i} \eta\left(\mathbf{x}_{i}, \boldsymbol{\beta}\right) \mid V_{1 i} \leq Y_{i} \leq V_{2 i}\right]\right\},
\end{aligned}
$$

em que $\operatorname{Cov}(\mathrm{X}, \mathrm{Y})$ denota Covariância entre as variáveis aleatórias $X$ e $Y$. Essas expressões, para diferentes distribuições SMN, podem ser obtidas por meio dos Corolários 2 e 3 dados no Apêndice A.

\subsection{Estudos de simulação}

Para estudar o desempenho do nosso modelo e o algoritmo proposto, apresentamos três estudos de simulação. 
A primeira parte deste estudo mostra que as estimativas dos parâmetros com base no algoritmo ECME dos modelos SMN-NLCR, fornecem boas propriedades assintóticas.

O objetivo da segunda parte é mostrar a consistência dos erros padrão estimados para os efeitos fixos.

O desempenho do parâmetro estimado na presença de valores outliers, sobre a variável resposta, é apresentado no terceiro estudo de simulação. Os procedimentos computacionais foram implementados utilizando o software R (R Core Team, 2013).

\subsubsection{Simulação 1: Propriedades Assintóticas}

O objetivo deste estudo de simulação é avaliar as propriedades assintóticas das estimativas dos parâmetros obtidas com o algoritmo ECME, desenvolvido na Seção 4.3. Foi realizado um estudo de simulação Monte Carlo considerando a curva de crescimento não linear definida por,

$$
Y_{i}=\frac{\beta_{1}}{1+\exp \left(\beta_{2}+\beta_{3} x_{i}\right)}+\varepsilon_{i}, \quad i=1, \ldots, n,
$$

em que $\varepsilon_{i} \sim \operatorname{SMN}\left(0, \sigma^{2}, \nu\right)$. Utilizamos para a variável $x_{i}$ uma sequência de valores entre 0.1 e 20 , e esses valores foram fixados para todo o experimento. Os verdadeiros valores dos parâmetros foram considerados como sendo $\beta_{1}=330, \beta_{2}=6,5, \beta_{3}=-0,7$ e $\sigma^{2}=3$ e os diferentes tamanhos de amostras considerados foram $n=30,50,100,150,200,300,400$, 500, 700 e 800 .

Considerando os diferentes tamanhos da amostra e os valores dos parâmetros, foram geradas 500 amostras aleatórias do modelo SMN-NLCR, com um nível de censura de $p=$ $10 \%$ (i.e., $10 \%$ das observações em cada conjunto de dados foram censurados).

A principal ideia aqui é a avaliação dos valores médios do viés e do erro quadrático médio, denotado por EQM. Assim, para $\beta_{i}$ para $i=1,2,3$, estas quantidades são definidas, respectivamente, por

$$
\text { Viés }\left(\widehat{\beta}_{i}\right)=\frac{1}{500} \sum_{j=1}^{500}\left(\widehat{\beta}_{i}^{(j)}-\beta_{i}\right) \quad \text { e } \operatorname{EQM}\left(\widehat{\beta}_{i}\right)=\frac{1}{500} \sum_{j=1}^{500}\left(\widehat{\beta}_{i}^{(j)}-\beta_{i}\right)^{2} \text {, }
$$

em que $\widehat{\beta}_{i}^{(j)}$ é o valor estimado de $\beta_{i}$ para a $j$-ésima amostra.

Da Figura 4.1, observamos que o Viés e o EQM médio convergem para zero quando o tamanho da amostra $n$ aumenta. Isto é, os resultados indicam que as estimativas de MV do modelo fornecem boas propriedades assintóticas.

\subsubsection{Simulação 2: Performance dos Erros Padrões}

O modelo considerado neste estudo de simulação é o mesmo que foi utilizado na Subseção 5.7.2. Neste estudo de simulação, avaliamos a consistência do método de aproximação dos erros padrões (EP) das estimativas de MV dos parâmetros de regressão, sugerido na Seção 4.4. Para o qual, geramos $k=1,000$ amostras de tamanho $n=150$, dos quatro diferentes 

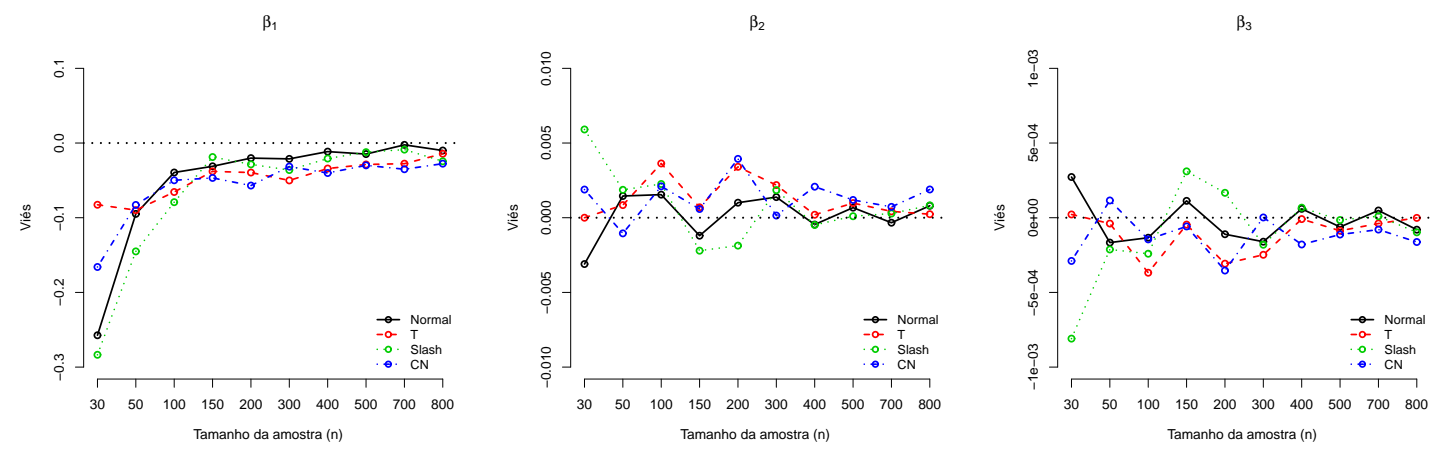

$\beta_{1}$
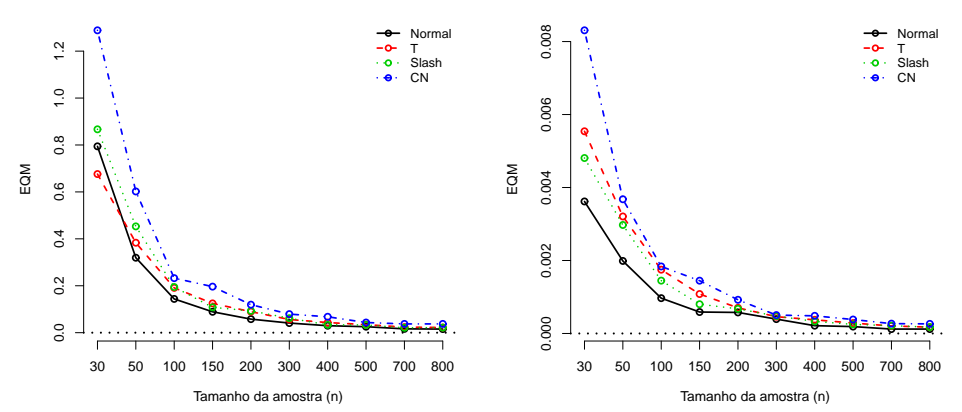

$\beta_{3}$

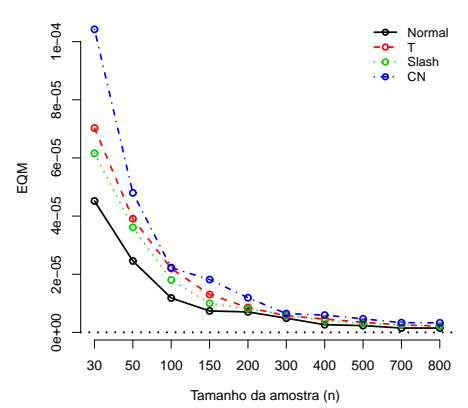

Figura 4.1: Viés e EQM médio para os parâmetros estimados

modelos SMN-NLCR, incluindo os modelos N-NLCR, T-NLCR com $\nu=4$, SL-NLCR com $\nu=3$ e CN-NLCR com $\boldsymbol{\nu}=(0,1,0,1)$. Para cada amostra, foram considerados cinco diferentes níveis de censura $(5 \%, 10 \%, 15 \%, 20 \%$ e $30 \%)$.

Para cada amostra gerada, da combinação do modelo SMN e o nível de censura, calculamos as estimativas de MV de $\boldsymbol{\beta}$, o seu correspondente EP e um intervalo de confiança assintótico (assumindo normalidade) de $95 \%$.

Na Tabela 4.1 apresentamos o erro padrão amostral de $\widehat{\beta}_{i}$, definido por:

$$
\mathrm{MC} \mathrm{SE}=\frac{1}{k-1} \sum_{j=1}^{k}\left(\widehat{\beta}_{i}^{(j)}-\widehat{\widehat{\beta}}_{i}\right)^{2}, \quad \text { where } \bar{\beta}_{i}=\frac{1}{k} \sum_{j=1}^{k} \widehat{\beta}_{i}^{(j)},
$$

assim como IM MC EP, que representa o valor médio (considerando as 1000 amostras) dos erros padrões obtidos utilizando o método da informação e a percentagem de cobertura, denotada por COV MC, a qual é obtida calculando a percentagem de vezes que os intervalos de confiança cobrem o verdadeiro valor do parâmetro.

Os resultados resumidos na Tabela 4.1 sugerem que a aproximação produzida pelo método de informação é satisfatório, independentemente do nível de censura e do modelo SMN considerado.

\subsubsection{Simulação 3: Desempenho das estimativas de MV}

O objetivo deste estudo é comparar o desempenho das estimativas de MV dos parâmetros dos modelos de regressão SMN-NLCR, na presença de valores outliers na variável resposta. 
Tabela 4.1: MC EP, MC IM EP e COV MC de $\widehat{\beta}_{i}$.

\begin{tabular}{|c|c|c|c|c|c|c|c|}
\hline \multirow{2}{*}{$\begin{array}{l}\text { Nível de } \\
\text { Censura }\end{array}$} & \multirow[t]{2}{*}{ Medida } & \multicolumn{3}{|c|}{ N-NLCR } & \multicolumn{3}{|c|}{ T-NLCR } \\
\hline & & $\widehat{\beta}_{1}$ & $\widehat{\beta}_{2}$ & $\widehat{\beta}_{3}$ & $\widehat{\beta}_{1}$ & $\widehat{\beta}_{2}$ & $\widehat{\beta}_{3}$ \\
\hline \multirow{3}{*}{$5 \%$} & MC EP & 0,2885 & 0,0251 & 0,0027 & 0,3412 & 0,0317 & 0,0035 \\
\hline & MC IM EP & 0,2682 & 0,0247 & 0,0027 & 0,3135 & 0,0290 & 0,0032 \\
\hline & $\mathrm{COV} \mathrm{MC}$ & $93,6 \%$ & $94,0 \%$ & $94,7 \%$ & $92,7 \%$ & $93,4 \%$ & $93,0 \%$ \\
\hline \multirow{3}{*}{$10 \%$} & MC EP & 0,2985 & 0,0273 & 0,0030 & 0,3564 & 0,0326 & 0,0036 \\
\hline & MC IM EP & 0,2656 & 0,0245 & 0,0027 & 0,3102 & 0,0287 & 0,0031 \\
\hline & $\mathrm{COV} \mathrm{MC}$ & $92,2 \%$ & $93,2 \%$ & $92,4 \%$ & $91,8 \%$ & $91,1 \%$ & $90,1 \%$ \\
\hline \multirow{3}{*}{$15 \%$} & MC EP & 0,3078 & 0,0264 & 0,0029 & 0,3539 & 0,0305 & 0,0033 \\
\hline & MC IM EP & 0,2651 & 0,0245 & 0,0027 & 0,3108 & 0,0288 & 0,0031 \\
\hline & $\mathrm{COV} \mathrm{MC}$ & $91,0 \%$ & $93,4 \%$ & $93,1 \%$ & $91,5 \%$ & $93.6 \%$ & $93,9 \%$ \\
\hline \multirow{3}{*}{$20 \%$} & MC EP & 0,3248 & 0,0280 & 0,0031 & 0,3607 & 0,0335 & 0,0036 \\
\hline & MC IM EP & 0,2641 & 0,0244 & 0,0027 & 0.3098 & 0.0286 & 0,0031 \\
\hline & $\mathrm{COV} \mathrm{MC}$ & $90.0 \%$ & $92,0 \%$ & $91,2 \%$ & $91,3 \%$ & $89,2 \%$ & $90,1 \%$ \\
\hline \multirow{3}{*}{$30 \%$} & MC EP & 0,3356 & 0,0295 & 0,0032 & 0,3986 & 0,0363 & 0,0040 \\
\hline & MC IM EP & 0,2637 & 0,0244 & 0,0027 & 0,3104 & 0,0288 & 0,0031 \\
\hline & $\mathrm{COV} \mathrm{MC}$ & $88,5 \%$ & $89,2 \%$ & $89,1 \%$ & $88,2 \%$ & $89,8 \%$ & $89,1 \%$ \\
\hline \multirow{2}{*}{$\begin{array}{l}\text { Nível de } \\
\text { censura }\end{array}$} & \multirow[t]{2}{*}{ Medida } & \multicolumn{3}{|c|}{ SL-NLCR } & \multicolumn{3}{|c|}{ CN-NLCR } \\
\hline & & $\widehat{\beta}_{1}$ & $\widehat{\beta}_{2}$ & $\widehat{\beta}_{3}$ & $\widehat{\beta}_{1}$ & $\widehat{\beta}_{2}$ & $\widehat{\beta}_{3}$ \\
\hline \multirow{3}{*}{$5 \%$} & MC EP & 0,3443 & 0,0302 & 0,0033 & 0,5131 & 0,0475 & 0,0053 \\
\hline & MC IM EP & 0,3253 & 0,0299 & 0,0033 & 0,4207 & 0,0389 & 0,0043 \\
\hline & $\mathrm{COV} \mathrm{MC}$ & $94,2 \%$ & $95,0 \%$ & $95,4 \%$ & $92,9 \%$ & $94,3 \%$ & $94,1 \%$ \\
\hline \multirow{3}{*}{$10 \%$} & MC EP & 0,3595 & 0,0312 & 0,0034 & 0,5324 & 0,0445 & 0,0049 \\
\hline & MC IM EP & 0,3269 & 0,0301 & 0,0033 & 0,4218 & 0,0391 & 0,0043 \\
\hline & $\mathrm{COV} \mathrm{MC}$ & $93,0 \%$ & $93,3 \%$ & $93,9 \%$ & $92,3 \%$ & $94,6 \%$ & $94,4 \%$ \\
\hline \multirow{3}{*}{$15 \%$} & MC EP & 0,3679 & 0,0335 & 0,0037 & 0,5846 & 0,0501 & 0,0055 \\
\hline & MC IM Ep & 0,3255 & 0,0301 & 0,0033 & 0,4272 & 0,0396 & 0,0043 \\
\hline & $\mathrm{COV} \mathrm{MC}$ & $92,0 \%$ & $91,9 \%$ & $91,8 \%$ & $90 \mathrm{~h}, 5 \%$ & $92,0 \%$ & $92,4 \%$ \\
\hline \multirow{3}{*}{$20 \%$} & MC EP & 0,3599 & 0,0326 & 0,0036 & 0,6326 & 0,0531 & 0,0058 \\
\hline & MC IM EP & 0,3277 & 0,0304 & 0,0033 & 0,4298 & 0,0400 & 0,0044 \\
\hline & $\mathrm{COV} \mathrm{MC}$ & $93,1 \%$ & $92,8 \%$ & $93,5 \%$ & $89,6 \%$ & $90,5 \%$ & $89,8 \%$ \\
\hline \multirow{3}{*}{$30 \%$} & MC EP & 0,3871 & 0,0368 & 0,0040 & 0,6106 & 0,0526 & 0,0057 \\
\hline & MC IM EP & 0,3309 & 0,0306 & 0,0034 & 0,4170 & 0,0387 & 0,0042 \\
\hline & $\mathrm{COV} \mathrm{MC}$ & $89,8 \%$ & $89,7 \%$ & $89,7 \%$ & $87,3 \%$ & $88,4 \%$ & $87,5 \%$ \\
\hline
\end{tabular}

Consideramos o modelo não linear Michaelis-Menten, definido em Vanegas et al. (2012)

$$
Y_{i}=\frac{\beta_{1} x_{i}}{\beta_{2}+x_{i}}+\varepsilon_{i}, \quad i=1, \ldots, n,
$$

em que $\varepsilon_{i} \sim \operatorname{SMN}\left(0, \sigma^{2}, \nu\right)$.

Para este estudo de simulação foi considerado $\beta_{1}=3, \beta_{2}=0,5$ e $\sigma^{2}=1$, um tamanho da amostra de $n=300$ e um nível de censura de $p=8 \%$. A covariável $x$ foi gerada assumindo uma distribuição $U(0,1)$, e esses valores foram fixados para todo o experimento, e foram consideradas 500 réplicas Monte Carlo.

Para avaliar, de que forma as estimativas de MV são influenciadas pela presença de valores outliers, foram considerados seis diferentes percentagens de outliers presentes no conjunto de dados $(\vartheta=1 \%, 2 \%, 3 \%, 4 \%, 5 \%$ e 10\%), em que os valores outliers são obtidos substituindo a observação não censurada $y_{i}$, escolhida aleatoriamente, pelo valor $y_{i}+2 D P(y)$, em que $D P$ representa o desvio padrão do conjunto de dados.

Seguindo Fagundes et al. (2013), o desempenho das estimativas de MV é avaliada considerando a magnitude média do erro relativo (MMER), definida como

$$
\operatorname{MMER}=\frac{1}{3}\left\{\left|\frac{\widehat{\beta}_{1}(\vartheta)-\widehat{\beta}_{1}}{\widehat{\beta}_{1}}\right|+\left|\frac{\widehat{\beta}_{2}(\vartheta)-\widehat{\beta}_{2}}{\widehat{\beta}_{2}}\right|+\left|\frac{\widehat{\sigma}_{2}^{2}(\vartheta)-\widehat{\sigma}_{2}^{2}}{\widehat{\sigma}_{2}^{2}}\right|\right\},
$$


em que $\widehat{\theta}(\vartheta)$ representa a estimativa de $\mathrm{MV}$ de $\theta$ depois da contaminação $\vartheta$, $\operatorname{com} \theta=$ $\beta_{1}, \beta_{2}, \sigma^{2}$.

A Tabela 4.2 mostra o valor da média e o desvio padrão das MMER obtidas, considerando as 500 amostras, para os diferentes modelos SMN-NLCR.

No caso do modelo N-NLCR, observamos que a influencia aumenta quando a percentagem de outliers aumenta. Por outro lado, temos que nos modelos com caudas mais pesadas do que a Normal, isto é, o modelo T-NLCR, SL-NLCR e CN-NLCR, as medidas de MMER variam pouco, indicando que são mais robustos do que o modelo N-NLCR, para modelar um conjunto de dados na presença de observações discrepantes.

Tabela 4.2: Média e o Desvio Padrão (em parentese) do MMER.

\begin{tabular}{|c|c|c|c|c|c|c|c|c|}
\hline \multirow{3}{*}{$\begin{array}{c}\% \text { de } \\
\text { Outliers } \\
1\end{array}$} & \multicolumn{8}{|c|}{ Modelos } \\
\hline & \multicolumn{2}{|c|}{ N-NLCR } & \multicolumn{2}{|c|}{ T-NLCR } & \multicolumn{2}{|c|}{ S-NLCR } & \multicolumn{2}{|c|}{ CN-NLCR } \\
\hline & 0,06052 & $(0,0296)$ & 0,04578 & $(0,0314)$ & 0,02517 & $(0,0336)$ & 0,04797 & $(0,0481)$ \\
\hline 2 & 0,09498 & $(0,0406)$ & 0,06933 & $(0,0413)$ & 0,06085 & $(0,0497)$ & 0,07034 & $(0,0471)$ \\
\hline 3 & 0,13238 & $(0,0495)$ & 0,09428 & $(0,0464)$ & 0,09012 & $(0,0521)$ & 0,09613 & $(0,0648)$ \\
\hline 4 & 0,16531 & $(0,0584)$ & 0,11085 & $(0,0580)$ & 0,12058 & $(0,0716)$ & 0,11725 & $(0,0723)$ \\
\hline 5 & 0,19635 & $(0,0718)$ & 0,13419 & $(0,0690)$ & 0,14844 & $(0,0724)$ & 0,13914 & $(0,0814)$ \\
\hline 10 & 0,32163 & $(0,0763)$ & 0,26046 & $(0,0965)$ & 0,27139 & $(0,0764)$ & 0,25357 & $(0,0814)$ \\
\hline
\end{tabular}

\subsection{Aplicação: Calibração ultrassônica}

Nesta seção, aplicaremos nossas técnicas propostas para o conjunto de dados calibração ultrassônica, que foi previamente analisada por Lin et al. (2009). Estes dados são resultados de um estudo desenvolvido no NIST (National Institute of Standards and Technology), relacionadas com calibração ultrassônica em 214 observações. A variável resposta é a resposta ultrassônica $(Y)$ e a variável preditora é a distância do metal $(X)$.

Seguindo Lin et al. (2009), consideramos o seguinte modelo não-linear:

$$
Y_{i}=\frac{\exp \left(-\beta_{1} x_{i}\right)}{\beta_{2}+\beta_{3} x_{i}}+\varepsilon_{i}, \quad i=1, \cdots, 214 .
$$

Utilizamos a mesma função não linear e com o intuito de avaliar o desempenho das estimativas de MV dos parâmetros dos modelos SMN-NLCR. Escolhemos ao acaso $p=8 \%$ (18 observações) como nível de censura intervalar e substituímos cada observação selecionada, $Y_{j}$, pelo intervalo $\left(Y_{L_{j}}, Y_{U_{j}}\right)$, em que $Y_{L_{j}}=\max \left(0, Y_{j}-\frac{1}{4} D P(Y)\right), Y_{U_{j}}=Y_{j}+\frac{1}{4} D P(Y) \mathrm{e}$ $D P$ é o desvio padrão de $Y$. Assim, as observações $\sharp 13, \sharp 30, \sharp 49, \sharp 50, \sharp 61, \sharp 74, \sharp 79, \sharp 106$, $\sharp 110, \sharp 118, \sharp 130, \sharp 137, \sharp 147, \sharp 166, \sharp 185, \sharp 195, \sharp 204$ e $\sharp 213$ foram selecionadas como valores censurados (Veja Figura 4.2).

Considerando o conjunto de dados de calibração com as novas observações censuradas, 


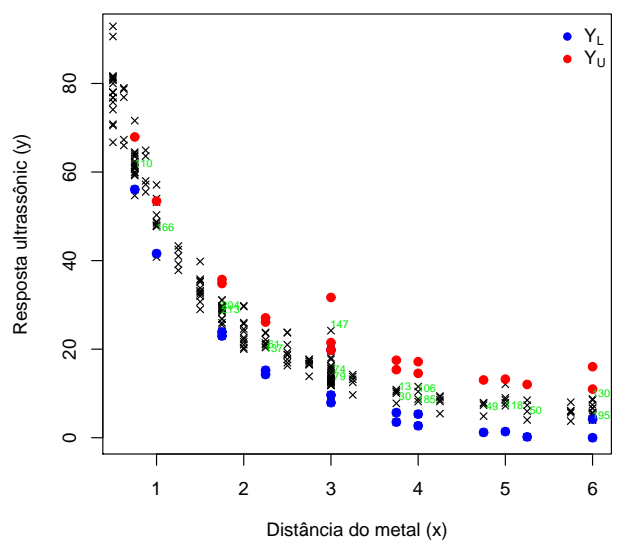

Figura 4.2: Gráfico de dispersão para os dados de calibração ultrassônica com censura

estimamos os parâmetros dos modelos SMN-NLCR, utilizando o algoritmo ECME descrito na Seção 4.3 e 4.4.

Na Tabela 4.3 apresentamos os parâmetros estimados e seus correspondentes erros padrões (EP), um intervalo de confiança assintótico de $95 \%$ e a significância dos parâmetros (* indica sinifcância do parâmetro).

Para os EP obtidos para os modelos T-NLCR, SL-NLCR e CN-NLCR são menores do que os obtidos com o modelo N-NLCR. Observe que as estimativas de MV de todos os coeficientes $\boldsymbol{\beta}$ são significativos para todos os modelos SMN-NLCR, uma vez que todos os intervalos de confiança de $95 \%$ não incluem zero. A Tabela 4.4 apresenta os critérios de seleção de modelos assim como o valor da Log verossimilhança para os modelos SMN-NLCR.

Os critérios AIC (Akaike, 1974), BIC (Schwarz, 1978), EDC (Bai et al., 1989) e AIC $S U R$ (Liang \& Zou, 2008) indicam que os três modelos com caudas mais pesadas do que a distribuição Normal produzem estimativas mais precisas. 


\begin{tabular}{|c|c|c|c|c|c|c|c|c|}
\hline \multirow{3}{*}{ Parâmetros } & \multicolumn{8}{|c|}{ Modelos } \\
\hline & \multicolumn{4}{|c|}{ N-NLCR } & \multicolumn{4}{|c|}{ T-NLCR } \\
\hline & Estimativa & $\mathrm{EP}$ & IC $(95 \%)$ & & Estimativa & EP & IC $(95 \%)$ & \\
\hline$\beta_{1}$ & 0,1953 & 0,0218 & $(0,1525 ; 0,2382)$ & * & 0,1803 & 0,0152 & $(0,1504 ; 0,2101)$ & * \\
\hline$\beta_{2}$ & 0,0061 & 0,0003 & $(0,0055 ; 0,0068)$ & * & 0,0059 & 0,0002 & $(0 . y 0054 ; 0,0064)$ & * \\
\hline$\beta_{3}$ & 0,0103 & 0,0007 & $(0,0088 ; 0,0119)$ & * & 0,0111 & 0,0005 & $(0,0100 ; 0 . y 0122)$ & * \\
\hline$\sigma^{2}$ & 11,1801 & - & - & & 3,6470 & - & - & \\
\hline$\nu$ & - & - & - & & 2,4562 & - & - & \\
\hline \multirow[t]{2}{*}{ Parâmetros } & \multicolumn{4}{|c|}{ SL-NLCR } & \multicolumn{4}{|c|}{ CN-NLCR } \\
\hline & Estimativa & EP & IC $(95 \%)$ & & Estimativa & EP & IC $(95 \%)$ & \\
\hline$\beta_{1}$ & 0,1846 & 0,0161 & $(0,1530 ; 0,2163)$ & * & 0,1868 & 0,0172 & $(0,1530 ; 0,2206)$ & * \\
\hline$\beta_{2}$ & 0,0060 & 0.y0002 & $(0,0054 ; 0,0065)$ & $*$ & 0,0060 & 0.y0002 & $(0,0055 ; 0,0065)$ & * \\
\hline$\beta_{3}$ & 0,0109 & $0 . y 0005$ & $(0,0098 ; 0,0121)$ & * & 0,01098 & 0,0006 & $(0,0095 ; 0 . y 0120)$ & * \\
\hline$\sigma^{2}$ & 2,1935 & - & - & & 4,7709 & - & - & \\
\hline$\nu$ & 1,0100 & - & - & & 0,2 & - & - & \\
\hline$\gamma$ & $\square$ & 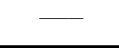 & 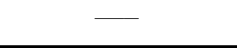 & & 0,2 & 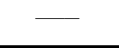 & 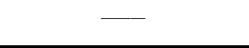 & \\
\hline
\end{tabular}

Tabela 4.3: Estimativa de MV, Erro padrão estimado (EP) e um intervalo de confiança assintótico (IC) para os modelos SMN-NLCR.

\begin{tabular}{cccccc}
\hline Modelo & log-verossimilhança & AIC & BIC & EDC & AIC $_{S U R}$ \\
\hline N-NLCR & $-520,783$ & 1049,566 & 1063,030 & 1053,269 & 1049,972 \\
T-NLCR & $\mathbf{- 4 9 7 , 1 0 6}$ & $\mathbf{1 0 0 4 , 2 1 0}$ & $\mathbf{1 0 2 1 , 0 4 2}$ & $\mathbf{1 0 0 8 , 8 4 1}$ & $\mathbf{1 0 0 4 , 7 5 6}$ \\
SL-NLCR & $-497,683$ & 1005,367 & 1022,197 & 1009,996 & 1005,911 \\
CN-NLCR & $-498,743$ & 1009,488 & 1029,684 & 1015,042 & 1010,190 \\
\hline
\end{tabular}

Tabela 4.4: Comparação entre os modelos SMN-NLCR.

Nestes primeiros quatro capítulos da tese, foram abordados de maneira detalhada os modelos de regressão linear e não linear univariados para dados censurados, sob Distribuições Simétricas a partir de uma perspectiva frequentista e Bayesiana, nos quais observa-se resultados bem interessantes, e que servirão de base para uma abordagem multivariada dos modelos de regressão linear para dados censurados. 


\section{Capítulo 5}

\section{Modelos lineares para dados correlacionados e censurados utilizando a distribuição t de Student}

\subsection{Introdução}

Em muitos estudos biomédicos e clínicos, o uso de modelos longitudinais tem mostrado um crescimento significativo nos últimos anos, tornando-se uma poderosa ferramenta para modelagem de respostas correlacionadas. Por exemplo, em ensaios clínicos de terapia antiretroviral em estudos de AIDS, as medidas do HIV-1 RNA (carga viral) são recolhidas ao longo de um período de tratamento para determinar as taxas de mudanças na quantidade do vírus. Estas medidas são utilizadas como um ponto chave, já que o acompanhamento da carga viral durante a terapia está disponível. Desta forma uma falha no tratamento pode ser determinada e um novo esquema de terapia pode ser recomendado (Ndembi et al., 2010).

Como para cada paciente são coletadas as medidas de carga viral ao longo do tempo, a estrutura de correlação entre as respostas devem ser levadas em consideração. Os modelos longitudinais permitem estimar as trajetórias da carga viral, assim como quantificar a estrutura de correlação entre as medidas da carga viral (veja Wu et al., 2010; Qiu \& $\mathrm{Wu}, 2010)$. No entanto, na prática, a modelagem estatística da carga viral pode ser um desafio devido aos seguintes problemas: (a) as medidas podem estar sujeitas a um limite superior ou inferior de quantificação. Como resultado, as observações podem ser censuradas à direita ou à esquerda, dependendo do ensaio de diagnóstico utilizado. Em geral, o limite pode variar de 400 copies $/ \mathrm{mL}$ para ensaios antigos para 40 copies $/ \mathrm{mL}$ para os ensaios mais atuais. (b) a carga viral é usualmente recolhida em tempos irregulares, por exemplo: muitas vezes os horários de medições variam de um tempo para outro, e os tempos normalmente 
estão associados com o grau da doença. (c) as medidas de carga viral, muitas vezes contêm observações discrepantes. Esta situação pode afetar tanto as estimativas dos parâmetros quanto o cálculo dos erros padrões, quando a suposição de normalidade é considerada. Portanto, um dos maiores desafios relacionados à modelagem de dados longitudinais em pesquisa sobre AIDS é considerar as características inerentes às medidas de carga viral.

Na literatura estatística e biomédica, os modelos de efeitos mistos lineares e não lineares baseados em suposições de normalidade são rotineiramente utilizados para modelar dados longitudinais (Laird \& H.Ware, 1982; Pinheiro \& Bates, 2000). No entanto, tal suposição pode não ser realista devido à presença de observações atípicas ou outliers. Assim, para contornar este problema, alguns modelos baseados em distribuições com caudas mais pesadas do que a da normal têm sido propostos. Por exemplo, Pinheiro et al. (2001) propuseram o modelo linear misto $t$ de Student, mostrando a sua robustez na presença de valores outliers. Outros autores, tais como Rosa et al. (2003), Lin \& Lee (2007), Meza et al. (2012), Lachos et al. (2013), centralizaram seus interesses em desenvolver estratégias para estimar os modelos lineares e não lineares de efeitos mistos, sob distribuições de caudas pesadas tais como a distribuição $t$ de Student, a slash e a normal contaminada.

Nos últimos anos, os modelos de regressão de efeitos mistos para respostas censuradas sob distribuições com caudas pesadas têm sido abordados em detalhe por Lachos et al. (2011), Bandyopadhyay et al. (2012), Arellano-Valle et al. (2012) e mais recentemente por Castro et al. (2014).

Atualmente foram propostos vários modelos considerando não só uma estrutura de correlação induzida pelo termo do efeito aleatório, mas também outro tipo de correlação no erro. Por exemplo, Wang (2013) estudou o modelo linear misto $t$ de Student multivariado ( $t$-LMM) para respostas obtidas em tempos irregulares utilizando uma estrutura de correlação de amortecimento exponencial parcimonioso (DEC). Este tipo de estrutura de correlação, proposto por Muñoz et al. (1992), leva em consideração a autocorrelação gerada intra-indivíduo para tempos irregulares. Além disso, Wang \& Fan (2011) estudaram o modelo linear misto $t$ de Student multivariado com estrutura de dependência AR(p) para os erros intra-indivíduos, no caso de respostas múltiplas.

No entanto, como mencionado por Goldstein et al. (1994) e Browne \& Goldstein (2010), nos casos em que as medidas repetidas são coletadas em tempos próximos ou se as correlações das observações ao longo do tempo não decaem rapidamente, os efeitos aleatórios podem não explicar adequadamente a dependência. Sendo assim, deve-se considerar uma estrutura de correlação mais complexa.

Neste sentido, seguindo Wang (2013), o objetivo deste Capítulo é analisar a estrutura de correlação DEC para a matriz de variância e covariância dos erros aleatórios, considerando o modelo de regressão censurado $t$ de Student multivariado. 


\subsection{Motivação: Dados UTI}

Nesta seção apresentaremos um conjunto de dados longitudinais baseado na interrupção do tratamento da terapia anti-retroviral não estruturada em adolescentes infectados com HIV de quatro instituições nos EUA. Neste caso, a carga viral de HIV-1 RNA é considerada como censurada quando for inferior ao limite de detecção do ensaio $(50$ copies $/ \mathrm{mL})$. É importante notar também a a possível presença de valores discrepantes no conjunto de dados.

Como mencionado inicialmente, este conjunto de dados descreve as medições da carga viral HIV-1 depois da interrupção do tratamento não estruturado (UTI) em 72 adolescentes americanos. UTI é definido como a interrupção do uso de todas as drogas anti retrovirais por algum período de tempo, após o qual o tratamento é retomado.

Saitoh et al. (2008) afirmaram que as principais razões para interromper o tratamento foram: (a) a fadiga, (b) pacientes que eram incapazes de usar medicamentos anti retrovirais, (c) toxicidade associada ao uso dos medicamento anti retrovirais e (d) efeitos adversos; dentre muitos outros.

Este conjunto de dados apresenta aproximadamente 7\% das observações com uma carga viral abaixo do limite de detecção de ensaio, isto é, $7 \%$ de observações censuradas à esquerda. A carga viral foi monitorada em tempos próximos de $0,1,3,6,9,12,18$ e 24 meses após a interrupção do tratamento, ou seja, foram coletados irregularmente ao longo do tempo. Mais detalhes sobre o conjunto de dados podem ser encontrados em Saitoh et al. (2008) e Vaida \& Liu (2009).

O perfil de cada indivíduo, segundo o valor da carga viral obtida para os diferentes tempos, após a UTI é apresentado na Figura 5.1 (painel a). Esta figura também mostra (painel b) os quantis da distribuição normal ( $Q Q$ Plot) dos resíduos obtidos ao estimar o modelo censurado de efeitos mistos, para o qual utilizamos o pacote lmec(.) (Vaida \& Liu, 2009). O $Q Q$ plot mostra um comportamento com caudas pesadas, sugerindo que a suposição de normalidade para os erros intra-indivíduo pode ser inapropriada. Além disso, a Tabela 5.1 mostra a correlação observada da carga viral em diferentes tempos em que podemos observar a presença de uma forte estrutura de correlação entre subsequentes medições da carga viral HVI-1.

Da Figura 5.1 também é possível perceber a presença de possíveis observações discrepantes ou outliers, as quais podem afetar as estimativas dos parâmetros e dos erros padrões. Sendo assim, é necessário propor modelos estatísticos mais apropriados, que possam contornar o problema da presença de possíveis valores discrepantes e que sejam mais robustos do que o modelo normal.

Uma alternativa seria, por exemplo considerar o modelo com distribuição $t$ de Student, o qual é mais robusto do que o modelo sob a distribuição normal, quando existem observaçãoes discrepantes. 
(a)

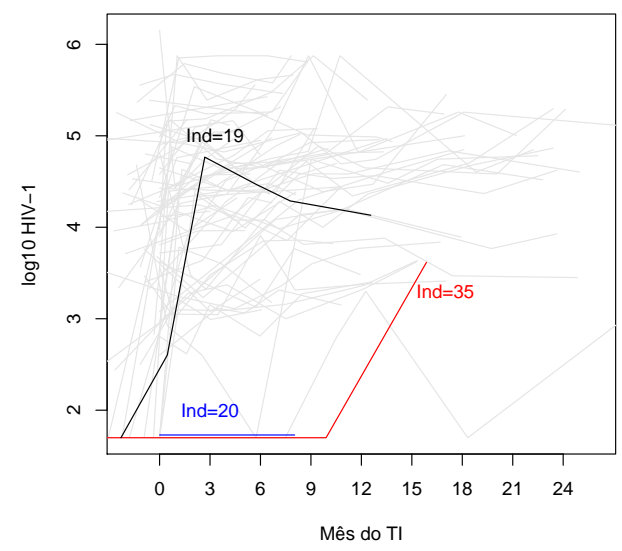

(b)

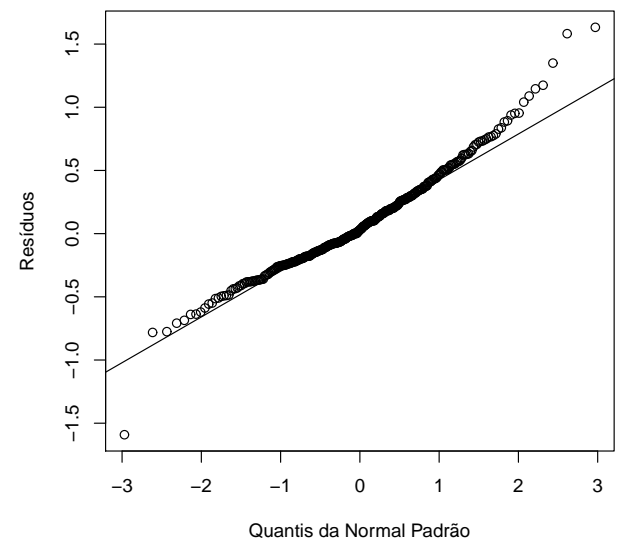

Figura 5.1: Dados UTI. (a) Perfil de cada indivíduo (na escala $\log _{10}$ ) segundo a carga viral. A trajetória de três indivíduos censurados são marcadas. (b) $Q Q$ plot para os resíduos do modelo normal.

\begin{tabular}{lccccccccc}
\hline & \multicolumn{10}{c}{$\log _{10} \mathbf{H I V - 1}$} \\
\cline { 3 - 9 } & & mês 0 & mês 1 & mês 3 & mês 6 & mês 9 & mês 12 & mês 18 & mês 24 \\
\hline & mês 0 & & 0,4877 & 0,4100 & 0,4052 & 0,4820 & 0,4435 & 0,3441 & 0,6529 \\
& mês 1 & 0,4877 & & 0,9145 & 0,8551 & 0,8455 & 0,6978 & 0,7090 & 0,6140 \\
$\log _{10} \mathbf{H I V - 1}$ & mês 3 & 0,4100 & 0,9145 & & 0,9255 & 0,8638 & 0,7209 & 0,7601 & 0,6301 \\
& mês 6 & 0,4052 & 0,8551 & 0,9255 & & 0,8238 & 0,6490 & 0,6548 & 0,5314 \\
& mês 9 & 0,4820 & 0,8455 & 0,8638 & 0,8238 & & 0,9185 & 0,7642 & 0,8061 \\
& mês 12 & 0,4435 & 0,6978 & 0,7209 & 0,6490 & 0,9185 & & 0,6646 & 0,6897 \\
& mês 18 & 0,3441 & 0,7090 & 0,7601 & 0,6548 & 0,7642 & 0,6646 & & 0,8947 \\
& mês 24 & 0,6529 & 0,6140 & 0,6301 & 0,5314 & 0,8061 & 0,6897 & 0,8947 & \\
\hline
\end{tabular}

Tabela 5.1: Correlação obervada do $\log _{10} \mathrm{HIV}-1$ em diferentes tempos de avaliação.

\subsection{Especificação do modelo}

\subsubsection{Preliminares}

Nesta seção apresentaremos alguns resultados úteis associados à distribuição t de Student $p$-variada os quais utilizaremos para a implementação do nosso algoritmo tipo EM.

A função de densidade (fdp) t de Student para o vetor aleatório $\mathbf{Y} \in \mathbb{R}^{p}$ com vetor de locação $\boldsymbol{\mu}$, matriz de escala $\boldsymbol{\Sigma}$ e graus de liberdade $\nu$, denotada por $t_{p}(. \mid \boldsymbol{\mu}, \boldsymbol{\Sigma}, \nu)$, é dada por

$$
t_{p}(\mathbf{y} \mid \boldsymbol{\mu}, \boldsymbol{\Sigma}, \nu)=\frac{\Gamma\left(\frac{p+\nu}{2}\right)}{\Gamma\left(\frac{\nu}{2}\right) \pi^{p / 2}} \nu^{-p / 2}|\boldsymbol{\Sigma}|^{-1 / 2}\left(1+\frac{d(\mathbf{y}, \boldsymbol{\theta})}{\nu}\right)^{-(p+\nu) / 2},
$$

em que $\Gamma$ (.) é a função gama e $d(\mathbf{y}, \boldsymbol{\theta})=(\mathbf{y}-\boldsymbol{\mu})^{\top} \boldsymbol{\Sigma}^{-1}(\mathbf{y}-\boldsymbol{\mu})$ é a distância de Mahalanobis para $\boldsymbol{\theta}=(\boldsymbol{\mu}, \boldsymbol{\Sigma}, \nu)$.

A fda de $\mathbf{Y}$ é denotada por $T_{p}(. \mid \boldsymbol{\mu}, \boldsymbol{\Sigma}, \nu)$ e é importante ressaltar que se $\nu>1, \boldsymbol{\mu}$ é a média e se $\nu>2, \nu(\nu-2)^{-1} \boldsymbol{\Sigma}$ é a matriz de variância e covariância. 
Note que quando $\nu$ tende ao infinito, $\mathbf{Y}$ segue aproximadamente uma distribuição normal multivariada com média $\boldsymbol{\mu}$ e matriz de covariância $\boldsymbol{\Sigma}$.

Uma importante propriedade do vetor aleatório $\mathbf{Y}$ é que este pode ser escrito como uma mistura de um vetor aleatório normal e uma variável aleatória positiva:

$$
\mathbf{Y}=\boldsymbol{\mu}+U^{-1 / 2} \mathbf{Z}
$$

em que $\mathbf{Z} \sim N_{p}(\mathbf{0}, \boldsymbol{\Sigma})$ é independente de $U$, uma variável aleatória positiva com distribuição $\operatorname{Gamma}(\nu / 2, \nu / 2)^{1}$.

A distribuição de $\mathbf{Y}$, restrita ao hiperplano definido por

$$
\mathbb{A}=\left\{\mathbf{y} \in \Re^{p} \mid \mathbf{y} \leq \mathbf{a}\right\}, \quad \text { com } \mathbf{y}=\left(y_{1}, \ldots, y_{p}\right)^{\top} \text { and } \mathbf{a}=\left(a_{1}, \ldots, a_{p}\right)^{\top},
$$

é a distribuição t de Student truncada, denotada por $T t_{p}(\boldsymbol{\mu}, \boldsymbol{\Sigma}, \nu ; \mathbb{A})$.

$\operatorname{Assim} \mathbf{Y} \mid \mathbf{Y} \in \mathbb{A} \sim T t_{p}(\boldsymbol{\mu}, \boldsymbol{\Sigma}, \nu ; \mathbb{A})$ e sua fdp é dada por $f(\mathbf{y} \mid \boldsymbol{\mu}, \boldsymbol{\Sigma}, \nu ; \mathbb{A})=\frac{t_{p}(\mathbf{y} \mid \boldsymbol{\mu}, \boldsymbol{\Sigma}, \nu)}{T_{p}(\mathbf{a} \mid \boldsymbol{\mu}, \boldsymbol{\Sigma}, \nu)} \mathbb{I}_{\mathbb{A}}(\mathbf{y})$, em que $\mathbb{I}_{\mathbb{A}}(\mathbf{y})$ é a função indicadora de $\mathbb{A}$.

Como foi mencionado no início desta Seção, as seguintes propriedades das distribuições $\mathrm{t}$ de Student e t de Student truncada são úteis para a implementação de nosso algoritmo EM. Assim, começaremos com a decomposição marginal condicional de um vetor aleatório t de Student. Veja detalhes da demonstração em Arellano-Valle \& Bolfarine (1995).

Proposição 2. Seja $\mathbf{Y} \sim t_{p}(\boldsymbol{\mu}, \boldsymbol{\Sigma}, \nu) \operatorname{com} \boldsymbol{\theta}=(\boldsymbol{\mu}, \boldsymbol{\Sigma}, \nu)$, particionada como $\mathbf{Y}^{\top}=\left(\mathbf{Y}_{1}^{\top}, \mathbf{Y}_{2}^{\top}\right)^{\top}$, $\operatorname{com} \operatorname{dim}\left(\mathbf{Y}_{1}\right)=p_{1}, \operatorname{dim}\left(\mathbf{Y}_{2}\right)=p_{2}, p_{1}+p_{2}=p$, em que $\boldsymbol{\Sigma}=\left(\begin{array}{ll}\boldsymbol{\Sigma}_{11} & \boldsymbol{\Sigma}_{12} \\ \boldsymbol{\Sigma}_{21} & \boldsymbol{\Sigma}_{22}\end{array}\right)$ e $\boldsymbol{\mu}=$ $\left(\boldsymbol{\mu}_{1}^{\top}, \boldsymbol{\mu}_{2}^{\top}\right)^{\top}$, são as correspondentes particições de $\boldsymbol{\Sigma}$ e $\boldsymbol{\mu}$. Então, temos que

(i) $\mathbf{Y}_{1} \sim t_{p_{1}}\left(\boldsymbol{\mu}_{1}, \boldsymbol{\Sigma}_{11}, \nu\right) \operatorname{com} \boldsymbol{\theta}_{1}=\left(\boldsymbol{\mu}_{1}, \boldsymbol{\Sigma}_{11}, \nu\right)$;

(ii) A fda de $\mathbf{Y}_{2} \mid \mathbf{Y}_{1}=\mathbf{y}_{1}$ é dada por

$$
P\left(\mathbf{Y}_{2} \leq \mathbf{y}_{2} \mid \mathbf{Y}_{1}=\mathbf{y}_{1}\right)=T_{p_{2}}\left(\mathbf{y}_{2} \mid \boldsymbol{\mu}_{2.1}, \widetilde{\boldsymbol{\Sigma}}_{22.1}, \nu+p_{1}\right)
$$

em que $\widetilde{\boldsymbol{\Sigma}}_{22.1}=\left(\frac{\nu+d\left(\mathbf{y}_{1}, \boldsymbol{\theta}_{1}\right)}{\nu+p_{1}}\right) \boldsymbol{\Sigma}_{22.1}, \quad d\left(\mathbf{y}_{1}, \boldsymbol{\theta}_{1}\right)=\left(\mathbf{y}_{1}-\boldsymbol{\mu}_{1}\right)^{\top} \boldsymbol{\Sigma}_{11}^{-1}\left(\mathbf{y}_{1}-\boldsymbol{\mu}_{1}\right), \quad \boldsymbol{\Sigma}_{22.1}=$ $\boldsymbol{\Sigma}_{22}-\boldsymbol{\Sigma}_{21} \boldsymbol{\Sigma}_{11}^{-1} \boldsymbol{\Sigma}_{12}$, e $\boldsymbol{\mu}_{2.1}=\boldsymbol{\mu}_{2}+\boldsymbol{\Sigma}_{21} \boldsymbol{\Sigma}_{11}^{-1}\left(\mathbf{y}_{1}-\boldsymbol{\mu}_{1}\right)$.

O seguinte resultado fornece os momentos de um vetor aleatório com distribuição t de Student truncada. As provas das Proposições 3 e 4 são apresentadas no Apêndice F e a demonstração da Proposição 5 é dada em Lin et al. (2011).

Proposição 3. Se $\mathbf{Y} \sim T t_{p}(\boldsymbol{\mu}, \boldsymbol{\Sigma}, \nu ; \mathbb{A}), \operatorname{com} \boldsymbol{\theta}=(\boldsymbol{\mu}, \boldsymbol{\Sigma}, \nu)$ e $\mathbb{A}$ definido em (5.1), então o $k$-ésimo momento de $\mathbf{Y}$, para $k=0,1,2$, é dado por

$$
E\left[\left(\frac{\nu+p}{\nu+d(\mathbf{y}, \boldsymbol{\theta})}\right)^{r} \mathbf{Y}^{(k)}\right]=c_{p}(\nu, r) \frac{T_{p}\left(\mathbf{a} \mid \boldsymbol{\mu}, \boldsymbol{\Sigma}^{*}, \nu+2 r\right)}{T_{p}(\mathbf{a} \mid \boldsymbol{\mu}, \mathbf{\Sigma}, \nu)} E_{\mathbf{W}}\left[\mathbf{W}^{(k)}\right]
$$

\footnotetext{
${ }^{1} \operatorname{Gamma}(a, b)$ denota a distribuição gamma com média $a / b$.
} 
em que $\mathbf{W} \sim T t_{p}\left(\boldsymbol{\mu}, \boldsymbol{\Sigma}^{*}, \nu+2 r ; \mathbb{A}\right), c_{p}(\nu, r)=\left(\frac{\nu+p}{\nu}\right)^{r}\left(\frac{\Gamma\left(\frac{\nu+p}{2}\right) \Gamma\left(\frac{\nu+2 r}{2}\right)}{\Gamma\left(\frac{\nu}{2}\right) \Gamma\left(\frac{\nu+p+2 r}{2}\right)}\right), d(\mathbf{y}, \boldsymbol{\theta})=$ $(\mathbf{Y}-\boldsymbol{\mu})^{\top} \boldsymbol{\Sigma}^{-1}(\mathbf{Y}-\boldsymbol{\mu}), \quad \boldsymbol{\Sigma}^{*}=\frac{\nu}{\nu+2 r} \boldsymbol{\Sigma}, \quad \mathbf{Y}^{(0)}=1, \mathbf{Y}^{(1)}=\mathbf{Y}, \mathbf{Y}^{(2)}=\mathbf{Y} \mathbf{Y}^{\top}$ e $\nu+2 r>0$.

Proposição 4. Seja $\mathbf{Y} \sim T t_{p}(\boldsymbol{\mu}, \boldsymbol{\Sigma}, \nu ; \mathbb{A}) \operatorname{com} \boldsymbol{\theta}=(\boldsymbol{\mu}, \boldsymbol{\Sigma}, \nu)$ e $\mathbb{A}$ definido em (5.1). Considere a partição $\mathbf{Y}^{\top}=\left(\mathbf{Y}_{1}^{\top}, \mathbf{Y}_{2}^{\top}\right) \operatorname{com} \operatorname{dim}\left(\mathbf{Y}_{1}\right)=p_{1}, \operatorname{dim}\left(\mathbf{Y}_{2}\right)=p_{2}, p_{1}+p_{2}=p, e$ as correspondentes partições dos parâmetros $\boldsymbol{\mu}=\left(\boldsymbol{\mu}_{1}^{\top}, \boldsymbol{\mu}_{2}^{\top}\right)^{\top}, \boldsymbol{\Sigma}=\left(\begin{array}{ll}\boldsymbol{\Sigma}_{11} & \boldsymbol{\Sigma}_{12} \\ \boldsymbol{\Sigma}_{21} & \boldsymbol{\Sigma}_{22}\end{array}\right)$ e as partições de $\mathbf{a}=\left(\mathbf{a}^{y_{1}}, \mathbf{a}^{y_{2}}\right)$ e $\mathbb{A}=\left(\mathbb{A}^{y_{1}}, \mathbb{A}^{y_{2}}\right)$.

Então utilizando a Proposição 2, o k-ésimo momento de $\mathbf{Y}_{2}$ é dado por:

$E\left[\left(\frac{\nu+p}{\nu+d(\mathbf{y}, \boldsymbol{\theta})}\right)^{r} \mathbf{Y}_{2}^{(k)} \mid \mathbf{Y}_{1}\right]=\frac{d_{p}\left(\nu, p_{1}, r\right)}{\left(\nu+d\left(\mathbf{y}_{1}, \boldsymbol{\theta}_{1}\right)\right)^{r}} \frac{T_{p_{2}}\left(\mathbf{a}^{y_{2}} \mid \boldsymbol{\mu}_{2.1}, \widetilde{\boldsymbol{\Sigma}}_{22.1}^{*}, \nu+p_{1}+2 r\right)}{T_{p_{2}}\left(\mathbf{a}^{y_{2}} \mid \boldsymbol{\mu}_{2.1}, \widetilde{\boldsymbol{\Sigma}}_{22.1}, \nu+p_{1}\right)} E_{\mathbf{W}}\left[\mathbf{W}^{(k)}\right]$,

em que $\mathbf{W} \sim T t_{p_{2}}\left(\boldsymbol{\mu}_{2.1}, \widetilde{\boldsymbol{\Sigma}}_{22.1}^{*}, \nu+p_{1}+2 r ; \mathbb{A}^{y_{2}}\right), d(\mathbf{y}, \boldsymbol{\theta})=(\mathbf{Y}-\boldsymbol{\mu})^{\top} \boldsymbol{\Sigma}^{-1}(\mathbf{Y}-\boldsymbol{\mu})$,

$d\left(\mathbf{y}_{1}, \boldsymbol{\theta}_{1}\right)=\left(\mathbf{Y}_{1}-\boldsymbol{\mu}_{1}\right)^{\top} \boldsymbol{\Sigma}_{11}^{-1}\left(\mathbf{Y}_{1}-\boldsymbol{\mu}_{1}\right), \quad \widetilde{\boldsymbol{\Sigma}}_{22.1}^{*}=\left(\frac{\nu+d\left(\mathbf{y}_{1}, \boldsymbol{\theta}_{1}\right)}{\nu+2 r+p_{1}}\right) \boldsymbol{\Sigma}_{22.1}$,

$d_{p}\left(\nu, p_{1}, r\right)=(\nu+p)^{r}\left(\frac{\Gamma\left(\frac{\nu+p}{2}\right) \Gamma\left(\frac{\nu+p_{1}+2 r}{2}\right)}{\Gamma\left(\frac{\nu+p_{1}}{2}\right) \Gamma\left(\frac{\nu+p+2 r}{2}\right)}\right) e \nu+p_{1}+2 r>0$.

Proposição 5. Seja $\mathbf{Y} \sim T t_{p}\left(\boldsymbol{\mu}, \Sigma, \nu ; \mathbb{A}^{*}\right), \operatorname{com} \mathbb{A}^{*}=\left\{\mathbf{y} \in \mathbb{R}^{p} \mid \mathbf{a}^{*}<\mathbf{y} \leq \mathbf{b}^{*}\right\}$, em que $\mathbf{a}^{*}=\left(a_{1}^{*}, \ldots, a_{p}^{*}\right)^{\top}, \mathbf{b}^{*}=\left(b_{1}^{*}, \ldots, b_{p}^{*}\right)^{\top}, \quad \Sigma=\Lambda \mathbf{R} \Lambda$ e $\Lambda=\operatorname{Diag}\left(\sigma_{11}, \ldots, \sigma_{p p}\right)$ é uma matriz diagonal $p \times p$ cujos elementos são todos positivos. Temos que $\mathbf{W}=\Lambda^{-1}(\mathbf{Y}-\boldsymbol{\mu}) \sim$ $T t_{p}(\mathbf{0}, \mathbf{R}, \nu ; \mathbb{A})$, em que $\mathbf{a}=\Lambda^{-1}\left(\mathbf{a}^{*}-\boldsymbol{\mu}\right) e \mathbf{b}=\Lambda^{-1}\left(\mathbf{b}^{*}-\boldsymbol{\mu}\right)$. Portanto,

$$
\begin{gathered}
E[\mathbf{Y}]=\boldsymbol{\mu}+\Lambda E[\mathbf{W}] \\
E\left[\mathbf{Y} \mathbf{Y}^{\top}\right]=\boldsymbol{\mu} \boldsymbol{\mu}^{\top}+\Lambda E[\mathbf{W}] \boldsymbol{\mu}^{\top}+\mu E\left[\mathbf{W}^{\top}\right] \Lambda+\Lambda E\left[\mathbf{W} \mathbf{W}^{\top}\right] \Lambda^{\top}
\end{gathered}
$$

em que $E[\mathbf{W}]$ e $E\left[\mathbf{W} \mathbf{W}^{\top}\right]$ são dados em Lin et al. (2011).

\subsubsection{Descrição do modelo}

Nosso modelo linear t de Student multivariado ( $t$-ML) é definido por:

$$
\mathbf{Y}_{i}=\mathbf{X}_{i} \boldsymbol{\beta}+\boldsymbol{\epsilon}_{i}
$$

com $\boldsymbol{\epsilon}_{i} \sim t_{n_{i}}\left\{\mathbf{0}, \boldsymbol{\Sigma}_{i}, \nu\right\}$, em que $\mathbf{Y}_{i}=\left(Y_{i 1}, \ldots, Y_{i n_{i}}\right)^{\top}$ é um vetor $n_{i} \times 1$ de respostas contínuas para a $i$-ésima unidade da amostra em determinados momentos $\mathbf{t}_{i}=\left(t_{i 1}, \ldots, t_{i n_{i}}\right)^{\top}, \mathbf{X}_{i}$ é a matriz de desenho de ordem $n_{i} \times p$ correspondente ao vetor $p \times 1$ de efeitos fixos $\boldsymbol{\beta}$ e $\boldsymbol{\epsilon}_{i}$ é o vetor $n_{i} \times 1$ de erros aleatórios.

Como foi descrito na Seção 5.1, as medidas da carga viral do HIV-1 obtidas em cada indivíduo apresentam evidências de correlação serial. Portanto, para obter estimativas mais precisas para os parâmetros, consideraremos uma estrutura parcimoniosa para a matriz de dispersão $\boldsymbol{\Sigma}_{i}=\sigma^{2} \mathbf{E}_{i}$, em que a matriz $\mathbf{E}_{i}$ incorpora a estrutura de dependência no tempo. 
Assim, adotamos uma estrutura de correlação damped exponential (DEC) para $\boldsymbol{\Sigma}_{i}$ proposta por Muñoz et al. (1992). Esta estrutura de correlação nos permite manipular observações que não estão igualmente espaçadas e/ou que são não balanceadas e é definida como

$$
\boldsymbol{\Sigma}_{i}=\sigma^{2} \mathbf{E}_{i}=\sigma^{2}\left[\phi_{1}^{\left|t_{i j}-t_{i k}\right|^{\phi_{2}}}\right], i=1, \ldots, n, \mathrm{e}, j, k=1, \ldots, n_{i},
$$

em que $\mathbf{E}_{i}=\mathbf{E}_{i}\left(\boldsymbol{\phi}, \mathbf{t}_{i}\right)=\left[\phi_{1}^{\left|t_{i j}-t_{i k}\right|^{\phi_{2}}}\right]$ tal que o parâmetro de correlação $\phi_{1}$ descreve a autocorrelação entre as observações separadas pela distância absoluta entre os tempos e o parâmetro de amortecimento $\phi_{2}$ avalia a aceleração do decaimento da função de autocorrelação, definindo um modelo autorregressivo (AR) de tempos contínuos.

É importante notar que considerando a estrutura de correlação DEC, é possível obter diferentes estruturas de correlação. Por exemplo, para um valor positivo do parâmetro $\phi_{1}$ :

- Se $\phi_{2}=0$, então $\mathbf{E}_{i}$ é a estrutura de correlação simétrica.

- Se $0<\phi_{2}<1$, então $\mathbf{E}_{i}$ é a estrutura de correlação com taxa de decaimento entre a estrutura simétrica e o modelo AR de primeira ordem (AR(1)).

- Se $\phi_{2}=1$, então $\mathbf{E}_{i}$ é uma estrutura de correlação AR(1).

- Se $\phi_{2}>1$, então $\mathbf{E}_{i}$ é uma estrutura de correlação com taxa de decaimento mais rápida que a do modelo $\mathrm{AR}(1)$.

- Se $\phi_{2} \rightarrow \infty$, então $\mathbf{E}_{i}$ é a estrutura de correlação do modelo média móvel de ordem 1 $(\mathrm{MA}(1))$.

Para maiores detalhes e discussões sobre a estrutura de correlação DEC, recomendamos a leitura de Muñoz et al. (1992).

Do ponto de vista prático e com o intuito de evitar problemas computacionais na estimação da estrutura de correlação DEC, o espaço paramétrico de $\phi_{1}$ e $\phi_{2}$ é definido em $\left\{\left(\phi_{1}, \phi_{1}\right): 0<\phi_{1}<1, \phi_{2}>0\right\}$.

Para facilitar o cálculo em um espaço bidimensional, consideraremos somente valores positivos para $\phi_{1}$ e $\phi_{2}$. Sob esta suposição, a função de autocorrelação resultante é muito comum na maioria das aplicações biomédicas ou epidemiológicas.

Para modelar respostas censuradas, consideramos a abordagem proposta por Vaida \& Liu (2009). Neste caso, os dados observados para o $i$-ésimo indivíduo é dado por $\left(\mathbf{V}_{i}, \mathbf{C}_{i}\right)$, em que $\mathbf{V}_{i}$ representa o vetor de respostas não censuradas e $\mathbf{C}_{i}$ o vetor indicador de censura. Ou seja,

$$
y_{i j} \leq V_{i j} \quad \text { se } C_{i j}=1 \text {, e } y_{i j}=V_{i j} \quad \text { se } C_{i j}=0
$$

Note que assumimos o caso de censura à esquerda, mas a extensão para outros tipos de censura é imediata. 


\subsubsection{Função de log-verosimilhança}

Nesta seção apresentamos a função de log-verossimilhança do nosso modelo linear censurado t de Student multivariado, denotado por $t$-MLC.

Para obter a função de log-verossimilhança, primeiro trataremos separadamente os componentes observados e censurados de $\mathbf{y}_{i}$, i.e., $\mathbf{y}_{i}=\left(\mathbf{y}_{i}^{o^{\top}}, \mathbf{y}_{i}^{c^{\top}}\right)^{\top}$, com $C_{i j}=0$ para todos os elementos em $\mathbf{y}_{i}^{o}$, e $C_{i j}=1$ para todos os elementos em $\mathbf{y}_{i}^{c}$. Analogamente, escrevemos $\mathbf{V}_{i}=\operatorname{vec}\left(\mathbf{V}_{i}^{o}, \mathbf{V}_{i}^{c}\right)$, em que $\operatorname{vec}(\cdot)$ representa a função que une vetores ou matrizes que tem o mesmo número de colunas, com $\boldsymbol{\Sigma}_{i}=\left(\begin{array}{l}\boldsymbol{\Sigma}_{i}^{o o} \boldsymbol{\Sigma}_{i}^{o c} \\ \boldsymbol{\Sigma}_{i}^{c o} \boldsymbol{\Sigma}_{i}^{c c}\end{array}\right)$. Então, utilizando a Proposição 2, temos que $\mathbf{y}_{i}^{o} \sim t_{n_{i}^{o}}\left(\mathbf{X}_{i}^{o} \boldsymbol{\beta}, \boldsymbol{\Sigma}_{i}^{o o}, \nu\right)$ e $\mathbf{y}_{i}^{c} \mid \mathbf{y}_{i}^{o}, \sim t_{n_{i}^{c}}\left(\boldsymbol{\mu}_{i}^{c o}, \mathbf{S}_{i}^{c o}, \nu+n_{i}^{o}\right)$, em que

$$
\boldsymbol{\mu}_{i}^{c o}=\mathbf{X}_{i}^{c} \boldsymbol{\beta}+\boldsymbol{\Sigma}_{i}^{c o} \boldsymbol{\Sigma}_{i}^{o o-1}\left(\mathbf{y}_{i}^{o}-\mathbf{X}_{i}^{o} \boldsymbol{\beta}\right), \mathbf{S}_{i}^{c o}=\left(\frac{\nu+d\left(\mathbf{y}_{i}^{o}, \boldsymbol{\theta}^{o}\right)}{\nu+n_{i}^{o}}\right) \boldsymbol{\Sigma}_{i}^{c c . o},
$$

com $\boldsymbol{\Sigma}_{i}^{c c . o}=\boldsymbol{\Sigma}_{i}^{c c}-\boldsymbol{\Sigma}_{i}^{c o} \boldsymbol{\Sigma}_{i}^{o o-1} \boldsymbol{\Sigma}_{i}^{o c}$ e $d\left(\mathbf{y}_{i}^{o}, \boldsymbol{\theta}^{o}\right)=\left(\mathbf{y}_{i}^{o}-\mathbf{X}_{i}^{o} \boldsymbol{\beta}\right)^{\top} \boldsymbol{\Sigma}_{i}^{o o-1}\left(\mathbf{y}_{i}^{o}-\mathbf{X}_{i}^{o} \boldsymbol{\beta}\right)$. Portanto, a função de verossimilhança para o $i$-ésimo individuo é dada por

$$
\begin{aligned}
L_{i}(\boldsymbol{\theta} \mid \mathbf{y}) & =f\left(\mathbf{V}_{i} \mid \mathbf{C}_{i}, \boldsymbol{\theta}\right)=f\left(\mathbf{y}_{i}^{c} \leq \mathbf{V}_{i}^{c} \mid \mathbf{y}_{i}^{o}=\mathbf{V}_{i}^{o}, \boldsymbol{\theta}\right) f\left(\mathbf{y}_{i}^{o}=\mathbf{V}_{i}^{o} \mid \boldsymbol{\theta}\right) \\
& =T_{n_{i}^{c}}\left(\mathbf{V}_{i}^{c} \mid \boldsymbol{\mu}_{i}^{c o}, \mathbf{S}_{i}^{c o}, \nu+n_{i}^{o}\right) t_{n_{i}^{o}}\left(\mathbf{V}_{i}^{o} \mid \mathbf{X}_{i}^{o} \boldsymbol{\beta}, \boldsymbol{\Sigma}_{i}^{o o}, \nu\right)=L_{i} .
\end{aligned}
$$

Assim, a função de log-verossimilhança para os dados observados é dada por $\ell(\boldsymbol{\theta} \mid \mathbf{y})=$ $\sum_{i=1}^{n} \log \left(L_{i}\right)$.

É importante notar que esta função pode ser calculada em cada passo do algoritmo tipo EM sem custo computacional, desde que os valores de $L_{i}$ sejam calculados em cada passo E.

Assumimos que o grau de liberdade da distribuição t de Student é fixo. Para escolher o valor mais apropriado para este parâmetro utilizaremos a função de log-verossimilhança perfilada (veja Lange et al. (1989); Meza et al. (2012)). Consequentemente, nosso vetor de parâmetros a estimar será $\boldsymbol{\theta}=\left(\boldsymbol{\beta}^{\top}, \sigma^{2}, \boldsymbol{\phi}\right)^{\top} \operatorname{com} \boldsymbol{\phi}=\left(\phi_{1}, \phi_{2}\right)$. Esta suposição é baseada no trabalho de Lucas (1997), no qual o autor mostrou que o impacto dos valores extremos é controlado quando os graus de liberdade são fixos.

\subsection{O algoritmo EM}

Com o intuito de encontrar as estimativas de MV dos parâmetros do modelo $t$-MLC, utilizaremos uma extensão do algoritmo EM conhecido como algoritmo ECM, desenvolvido por Meng \& Rubin (1993). O uso do algoritmo ECM é justificado, uma vez que a presença de censura e da estrutura de correlação DEC no modelo (5.2)-(5.3) dificulta o cálculo computacional no passo M.

Uma característica chave deste algoritmo é que ele preserva a estabilidade do algoritmo EM com a vantagem de possuir uma taxa de convergência mais rápida do que a do algoritmo original. 
Com o objetivo de propor o algoritmo ECM para nosso modelo $t$-MLC, definimos em primeiro lugar $\mathbf{y}=\left(\mathbf{y}_{1}^{\top}, \ldots, \mathbf{y}_{n}^{\top}\right)^{\top}, \mathbf{u}=\left(u_{1}, \ldots, u_{n}\right)^{\top}, \mathbf{V}=\operatorname{vec}\left(\mathbf{V}_{1}, \ldots, \mathbf{V}_{n}\right)$, e $\mathbf{C}=$ $\operatorname{vec}\left(\mathbf{C}_{1}, \ldots, \mathbf{C}_{n}\right)$, de modo que para o $i$-ésimo indivíduo observa-se $\left(\mathbf{V}_{i}, \mathbf{C}_{i}\right)$.

Assim, tratamos u e y como hipotéticos "dados perdidos", aumentando os dados observados $\mathbf{V}, \mathbf{C}$ correspondente ao mecanismo de censura. Desta forma, definimos o vetor de dados completos como $\mathbf{y}_{c}=\left(\mathbf{C}^{\top}, \mathbf{V}^{\top}, \mathbf{y}^{\top}, \mathbf{u}^{\top}\right)^{\top}$.

Como se sabe, o algoritmo ECM deve ser aplicado para a função de log-verossimilhança completa obtida; a qual é dada por

$$
\ell_{c}\left(\boldsymbol{\theta} \mid \mathbf{y}_{c}\right)=\sum_{i=1}^{n} \ell_{i}\left(\boldsymbol{\theta} \mid \mathbf{y}_{c}\right)
$$

com

$\ell_{i}\left(\boldsymbol{\theta} \mid \mathbf{y}_{c}\right)=-\frac{1}{2}\left[n_{i} \log \left(\sigma^{2}\right)+\log \left(\left|\mathbf{E}_{i}\right|\right)+\frac{u_{i}}{\sigma^{2}}\left(\mathbf{y}_{i}-\mathbf{X}_{i} \boldsymbol{\beta}\right)^{\top} \mathbf{E}_{i}^{-1}\left(\mathbf{y}_{i}-\mathbf{X}_{i} \boldsymbol{\beta}\right)\right]+\log \left(h\left(u_{i} \mid \nu\right)\right)+c$,

em que $c$ é uma constante que não depende de $\boldsymbol{\theta}$ e $h\left(u_{i} \mid \nu\right)$ é a fdp $\operatorname{Gamma}(\nu / 2, \nu / 2)$.

Finalmente, o algoritmo ECM para o modelo $t$-MLC pode ser resumido através dos seguintes passos:

Passo E:

Dados os valores atuais de $\boldsymbol{\theta}=\widehat{\boldsymbol{\theta}}^{(k)}$, o passo E calcula a esperança condicional da função de log-verossimilhança completa

$$
Q\left(\boldsymbol{\theta} \mid \widehat{\boldsymbol{\theta}}^{(k)}\right)=\sum_{i=1}^{n} Q_{i}\left(\boldsymbol{\theta} \mid \widehat{\boldsymbol{\theta}}^{(k)}\right),
$$

em que

$$
Q_{i}\left(\boldsymbol{\beta}, \sigma^{2}, \boldsymbol{\phi} \mid \widehat{\boldsymbol{\theta}}^{(k)}\right)=-\frac{n_{i}}{2} \log \left(\sigma^{2}\right)-\frac{1}{2} \log \left(\left|\mathbf{E}_{i}\right|\right)-\frac{1}{2 \sigma^{2}} A_{i}^{(k)}(\boldsymbol{\beta}, \boldsymbol{\phi}),
$$

com

$$
A_{i}^{(k)}(\boldsymbol{\beta}, \boldsymbol{\phi})=\left[\operatorname{tr}\left({\widehat{u \mathbf{y}_{i}^{2}}}^{(k)} \mathbf{E}_{i}^{-1}\right)-2 \boldsymbol{\beta} \mathbf{X}_{i}^{\top} \mathbf{E}_{i}^{-1} \widehat{u \mathbf{y}}_{i}^{(k)}+\widehat{u}_{i}^{(k)} \boldsymbol{\beta} \mathbf{X}_{i}^{\top} \mathbf{E}_{i}^{-1} \mathbf{X}_{i} \boldsymbol{\beta}\right]
$$

Note que, como $\nu$ é considerado fixo, não precisamos obter $E\left[\log \left(h\left(u_{i} \mid \nu\right)\right) \mid \mathbf{V}, \mathbf{C}, \widehat{\boldsymbol{\theta}}^{(k)}\right]$.

\section{Passo CM:}

Neste passo, $Q\left(\boldsymbol{\theta} \mid \widehat{\boldsymbol{\theta}}^{(k)}\right)$ é condicionalmente maximizada sobre $\boldsymbol{\theta}$ e a nova estimação $\widehat{\boldsymbol{\theta}}^{(k+1)}$ é obtida. Especificamente, temos que

$$
\begin{aligned}
\widehat{\boldsymbol{\beta}}^{(k+1)} & =\left(\sum_{i=1}^{n} \widehat{u}_{i}^{(k)} \mathbf{X}_{i}^{\top}\left(\widehat{\mathbf{E}}_{i}^{(k)}\right)^{-1} \mathbf{X}_{i}\right)^{-1} \sum_{i=1}^{n} \mathbf{X}_{i}^{\top}\left(\widehat{\mathbf{E}}_{i}^{(k)}\right)^{-1} \widehat{u \mathbf{y}}_{i}^{(k)} ; \\
{\widehat{\sigma^{2}}}^{(k+1)} & =\frac{1}{N} \sum_{i=1}^{n} A_{i}^{(k)}\left(\widehat{\boldsymbol{\beta}}^{(k+1)}, \widehat{\boldsymbol{\phi}}^{(k)}\right) ; \\
\widehat{\boldsymbol{\phi}}^{(k+1)} & =\operatorname{argmax}_{\boldsymbol{\phi}}\left\{-\frac{1}{2} \sum_{i=1}^{n}\left[\log \left(\left|\widehat{\mathbf{E}}_{i}^{(k)}\right|\right)+A_{i}^{(k)}\left(\widehat{\boldsymbol{\beta}}^{(k+1)}, \boldsymbol{\phi}\right)\right]\right\},
\end{aligned}
$$


em que $N=\sum_{i=1}^{n} n_{i}$.

Este algoritmo é iterado até que algum critério de convergência seja satisfeito. Neste caso, adotamos a distância envolvendo duas avaliações sucessivas da função de log-verossimilhança, dada por $\left|\ell\left(\widehat{\boldsymbol{\theta}}^{(k+1)}\right) / \ell\left(\widehat{\boldsymbol{\theta}}^{(k)}\right)-1\right|$, como um critério de convergência.

É importante ressaltar que o passo E se reduz ao cálculo de ${\widehat{u \mathbf{y}_{i}^{2}}}^{2} \widehat{u \mathbf{y}}_{i}$, e $\widehat{u}_{i}$, os quais podem ser obtidos de forma fechada utilizando os resultados dados na Subseção 5.3.1. Portanto,

1. se todas as observações do $i$-ésimo indivíduo forem censuradas, da Proposição 3 :

$$
\begin{aligned}
\widehat{u \mathbf{y}_{i}^{2}} & =E\left[u_{i} \mathbf{y}_{i} \mathbf{y}_{i}^{\top} \mid \mathbf{V}_{i}, \mathbf{C}_{i}, \widehat{\boldsymbol{\theta}}\right]=\frac{T_{n_{i}}\left(\mathbf{V}_{i} \mid \widehat{\boldsymbol{\mu}}_{i}, \widehat{\boldsymbol{\Sigma}}_{i}^{*}, \nu+2\right)}{T_{n_{i}}\left(\mathbf{V}_{i} \mid \widehat{\boldsymbol{\mu}}_{i}, \widehat{\boldsymbol{\Sigma}}_{i}, \nu\right)} E\left[\mathbf{W}_{i} \mathbf{W}_{i}^{\top}\right] ; \\
\widehat{u \mathbf{y}}_{i} & =E\left[u_{i} \mathbf{y}_{i} \mid \mathbf{V}_{i}, \mathbf{C}_{i}, \widehat{\boldsymbol{\theta}}\right]=\frac{T_{n_{i}}\left(\mathbf{V}_{i} \mid \widehat{\boldsymbol{\mu}}_{i}, \widehat{\boldsymbol{\Sigma}}_{i}^{*}, \nu+2\right)}{T_{n_{i}}\left(\mathbf{V}_{i} \mid \widehat{\boldsymbol{\mu}}_{i}, \widehat{\boldsymbol{\Sigma}}_{i}, \nu\right)} E\left[\mathbf{W}_{i}\right] ; \\
\widehat{u}_{i} & =E\left[u_{i} \mid \mathbf{V}_{i}, \mathbf{C}_{i}, \widehat{\boldsymbol{\theta}}\right]=\frac{T_{n_{i}}\left(\mathbf{V}_{i} \mid \widehat{\boldsymbol{\mu}}_{i}, \widehat{\boldsymbol{\Sigma}}_{i}^{*}, \nu+2\right)}{T_{n_{i}}\left(\mathbf{V}_{i} \mid \widehat{\boldsymbol{\mu}}_{i}, \widehat{\boldsymbol{\Sigma}}_{i}, \nu\right)},
\end{aligned}
$$

em que $\mathbf{W}_{i} \sim T t_{n_{i}}\left(\widehat{\boldsymbol{\mu}}_{i}, \widehat{\boldsymbol{\Sigma}}_{i}^{*}, \nu+2 ; \mathbb{A}_{i}\right), \widehat{\boldsymbol{\mu}}_{i}=\mathbf{X}_{i} \widehat{\boldsymbol{\beta}}, \widehat{\boldsymbol{\Sigma}}_{i}^{*}=\frac{\nu}{\nu+2} \widehat{\boldsymbol{\Sigma}}_{i}, \widehat{\boldsymbol{\Sigma}}_{i}=\widehat{\sigma^{2}} \widehat{\mathbf{E}}_{i} \mathrm{e}$ $\mathbb{A}_{i}=\left\{\mathbf{W}_{i} \in \mathbb{R}^{n_{i}} \mid \mathbf{w}_{i} \leq \mathbf{V}_{i}\right\}$ em que $\mathbf{w}_{i}=\left(w_{i 1}, \ldots, w_{i n_{i}}\right)^{\top}$ e $\mathbf{V}_{i}=\left(V_{i 1}, \ldots, V_{i n_{i}}\right)^{\top} ;$

2. se todas as observações do $i$-ésimo indivíduo forem não censuradas, então:

$$
\widehat{u \mathbf{y}_{i}^{2}}=\frac{\nu+n_{i}}{\nu+d\left(\mathbf{y}_{i}, \boldsymbol{\theta}\right)} \mathbf{y}_{i} \mathbf{y}_{i}^{\top} ;{\widehat{u y_{i}}}=\frac{\nu+n_{i}}{\nu+d\left(\mathbf{y}_{i}, \boldsymbol{\theta}\right)} \mathbf{y}_{i} ; \widehat{u}_{i}=\frac{\nu+n_{i}}{\nu+d\left(\mathbf{y}_{i}, \boldsymbol{\theta}\right)},
$$

em que $d\left(\mathbf{y}_{i}, \boldsymbol{\theta}\right)=\left(\mathbf{y}_{i}-\mathbf{X}_{i} \boldsymbol{\beta}\right)^{\top} \boldsymbol{\Sigma}_{i}^{-1}\left(\mathbf{y}_{i}-\mathbf{X}_{i} \boldsymbol{\beta}\right)$;

3. se o $i$-ésimo indivíduo tem observações censuradas e não censuradas, então da Proposição 4 e dado que $\left\{\mathbf{y}_{i} \mid \mathbf{V}_{i}, \mathbf{C}_{i}\right\},\left\{\mathbf{y}_{i} \mid \mathbf{V}_{i}, \mathbf{C}_{i}, \mathbf{y}_{i}^{o}\right\}$, e $\left\{\mathbf{y}_{i}^{c} \mid \mathbf{V}_{i}, \mathbf{C}_{i}, \mathbf{y}_{i}^{o}\right\}$ são processos equivalentes, temos que:

$$
\begin{aligned}
\widehat{u \mathbf{y}_{i}^{2}} & =E\left[u_{i} \mathbf{y}_{i} \mathbf{y}_{i}^{\top} \mid \mathbf{y}_{i}^{o}, \mathbf{V}_{i}, \mathbf{C}_{i}, \widehat{\boldsymbol{\theta}}\right]=\left(\begin{array}{cc}
\mathbf{y}_{i}^{o} \mathbf{y}_{i}^{o \top} \widehat{u}_{i} & \widehat{u}_{i} \mathbf{y}_{i}^{o} \widehat{\mathbf{w}}_{i}^{c \top} \\
\widehat{u}_{i} \widehat{\mathbf{w}}_{i}^{c} \mathbf{y}_{i}^{o \top} & \widehat{u}_{i} \widehat{\mathbf{w}}_{i}^{2}
\end{array}\right) ; \\
\widehat{u \mathbf{y}_{i}} & =E\left[u_{i} \mathbf{y}_{i} \mid \mathbf{y}_{i}^{o}, \mathbf{V}_{i}, \mathbf{C}_{i}, \widehat{\boldsymbol{\theta}}\right]=\operatorname{vec}\left(y_{i}^{o} \widehat{u}_{i}, \widehat{\mathbf{w}}_{i}^{c}\right) \\
\widehat{u}_{i} & =E\left[u_{i} \mid \mathbf{y}_{i}^{o}, \mathbf{V}_{i}, \mathbf{C}_{i}, \widehat{\boldsymbol{\theta}}\right]=\left(\frac{n_{i}^{o}+\nu}{\nu+d\left(\mathbf{y}_{i}^{o}, \boldsymbol{\theta}^{o}\right)}\right) \frac{T_{p}\left(\mathbf{V}_{i} \mid \boldsymbol{\mu}_{i}^{c o}, \widetilde{\mathbf{S}}^{c o}, \nu+n_{i}^{o}+2\right)}{T_{p}\left(\mathbf{V}_{i} \mid \boldsymbol{\mu}_{i}^{c o}, \mathbf{S}^{c o}, \nu+n_{i}^{o}\right)}
\end{aligned}
$$

em que $\widetilde{\mathbf{S}}^{c o}=\left(\frac{\nu+d\left(\mathbf{y}_{i}^{o}, \boldsymbol{\theta}^{o}\right)}{\nu+2+n_{i}^{o}}\right) \boldsymbol{\Sigma}_{i}^{c c . o}, \widehat{\mathbf{w}}_{i}^{c}=E\left[\mathbf{W}_{i}\right]$, e $\widehat{\mathbf{w}}_{i}^{c}=E\left[\mathbf{W}_{i} \mathbf{W}_{i}^{\top}\right], \operatorname{com} \mathbf{W}_{i} \sim$ $T t_{n_{i}^{c}}\left(\boldsymbol{\mu}_{i}^{c o}, \widetilde{\mathbf{S}}^{c o}, \nu+n_{i}^{o}+2 ; \mathbb{A}_{i}^{c}\right)$ em que $\boldsymbol{\Sigma}_{i}^{c c . o}, \boldsymbol{\mu}_{i}^{c o}, d\left(\mathbf{y}_{i}^{o}, \boldsymbol{\theta}^{o}\right)$ e $\mathbf{S}^{c o}$ são definidas como em (5.5).

Como foi mencionado na Subseção 5.3.1, as fórmulas para $E[\mathbf{W}]$ e $E\left[\mathbf{W W}^{\top}\right]$, em que $\mathbf{W} \sim T t_{p}(\boldsymbol{\mu}, \boldsymbol{\Sigma}, \nu ; \mathbb{A})$, podem ser obtidas em Lin et al. (2011).

Para o cálculo da fda da distribuição t de Student multivariada, utilizamos a função $p m v t()$ do pacote mvtnorm (Genz et al., 2008) do software R. Finalmente, mais detalhes sobre nosso algoritmo ECM para o modelo t-MLC podem ser encontrados no Apêndice E. 


\subsubsection{Imputação dos casos censurados via o algoritmo ECM}

Seja $\mathbf{y}_{i}^{c}$ o vetor de observações censuradas, ou "parcialmente observadas", do $i$-ésimo indivíduo. Utilizando o algoritmo ECM podemos imputar estes valores "parcialmente observadas", que denotaremos por $\widetilde{\mathbf{y}}_{i}^{(c)}$, calculando

$$
\widetilde{\mathbf{y}}_{i}^{(c)}=E\left[\mathbf{y}_{i}^{c} \mid \mathbf{y}_{i}^{o}, \mathbf{V}_{i}, \mathbf{C}_{i}, \widehat{\boldsymbol{\theta}}\right],
$$

o qual pode ser obtido considerando dois possíveis cenários:

1. se todas as observações do $i$-ésimo indivíduo forem censuradas

$$
\widetilde{\mathbf{y}}_{i}^{(c)}=E\left[\mathbf{y}_{i}^{c} \mid \mathbf{V}_{i}, \mathbf{C}_{i}, \widehat{\boldsymbol{\theta}}\right],
$$

em que $\mathbf{y}_{i}^{c} \mid \mathbf{V}_{i}, \mathbf{C}_{i}, \widehat{\boldsymbol{\theta}} \sim T t_{n_{i}}\left(\mathbf{X}_{i} \widehat{\boldsymbol{\beta}}, \widehat{\boldsymbol{\Sigma}}_{i}, \nu ; \mathbb{A}_{i}\right) ; \operatorname{com} \mathbb{A}_{i}=\left\{\mathbf{y}_{i} \in \mathbb{R}^{n_{i}} \mid \mathbf{y}_{i} \leq \mathbf{a}_{i}\right\}, \quad \mathbf{y}_{i}=$ $\left(y_{i 1}, \ldots, y_{i n_{i}}\right)^{\top}$ e $\mathbf{a}_{i}=\left(a_{i 1}, \ldots, a_{i n_{i}}\right)^{\top}$ que será calculada utilizando a Proposição 5 ;

2. se o $i$-ésimo indivíduo tem observações censuradas e não censuradas, então da Proposição 4 com $r=0$ e $k=1$, temos que

$$
\widetilde{\mathbf{y}}_{i}^{(c)}=E\left[\mathbf{y}_{i}^{c} \mid \mathbf{y}_{i}^{o}, \mathbf{V}_{i}, \mathbf{C}_{i}, \widehat{\boldsymbol{\theta}}\right],
$$

em que $\mathbf{y}_{i}^{c} \mid \mathbf{y}_{i}^{o}, \sim t_{n_{i}^{c}}\left(\widehat{\boldsymbol{\mu}}_{i}^{c o}, \widehat{\mathbf{S}}_{i}^{c o}, \nu+n_{i}^{o}\right), \widehat{\boldsymbol{\mu}}_{i}^{c o}=\mathbf{X}_{i}^{c} \widehat{\boldsymbol{\beta}}+\widehat{\boldsymbol{\Sigma}}_{i}^{c o} \widehat{\boldsymbol{\Sigma}}_{i}^{o o-1}\left(\mathbf{y}_{i}^{o}-\mathbf{X}_{i}^{o} \widehat{\boldsymbol{\beta}}\right)$,

$\widehat{\mathbf{S}}_{i}^{c o}=\left(\frac{\nu+\widehat{d}\left(\mathbf{y}_{i}^{o}, \boldsymbol{\theta}^{o}\right)}{\nu+n_{i}^{o}}\right) \widehat{\mathbf{\Sigma}}_{i}^{c c . o}, \operatorname{com} \widehat{\boldsymbol{\Sigma}}_{i}^{c c . o}=\widehat{\boldsymbol{\Sigma}}_{i}^{c c}-\widehat{\boldsymbol{\Sigma}}_{i}^{c o} \widehat{\boldsymbol{\Sigma}}_{i}^{o o-1} \widehat{\boldsymbol{\Sigma}}_{i}^{o c} \mathrm{e}$

$\widehat{d}\left(\mathbf{y}_{i}^{o}, \boldsymbol{\theta}^{o}\right)=\left(\mathbf{y}_{i}^{o}-\mathbf{X}_{i}^{o} \widehat{\boldsymbol{\beta}}\right)^{\top} \widehat{\boldsymbol{\Sigma}}_{i}^{o o-1}\left(\mathbf{y}_{i}^{o}-\mathbf{X}_{i}^{o} \widehat{\boldsymbol{\beta}}\right)$.

É importante ressaltar que não foi considerado o caso no qual todas as observações do $i$-ésimo indivíduo forem não censuradas já que, nesta situação, as respostas deste indivíduo são "completamente observadas"e não é necessário utilizar o procedimento de imputação.

\subsection{Erro padrão aproximado para os efeitos fixos}

Com o objetivo de avaliar a variabilidade do estimador de MV de $\boldsymbol{\beta}$ para o modelo $t$ MLC, utilizaremos a metodologia desenvolvida na Seção 4.4 e utilizando os resultados dados em Lange et al. (1989), podemos encontrar uma aproximação assintótica para as variâncias dos efeitos fixos do modelo $t$-MLC. Essa aproximação é dada por

$$
\mathbf{J}_{\boldsymbol{\beta} \boldsymbol{\beta}}=\operatorname{Var}[\widehat{\boldsymbol{\beta}}]=\left(\sum_{i=1}^{n} \frac{\nu+n_{i}}{\nu+n_{i}+2} \mathbf{X}_{i}^{\top} \boldsymbol{\Sigma}_{i}^{-1} \mathbf{X}_{i}-\sum_{i=1}^{n} \mathbf{X}_{i}^{\top} \boldsymbol{\Sigma}_{i}^{-1} \mathbf{B}_{i} \boldsymbol{\Sigma}_{i}^{-1} \mathbf{X}_{i}\right)^{-1},
$$

em que $\mathbf{B}_{i}=\operatorname{Var}\left[\left(\frac{\nu+n_{i}}{\nu+d\left(\mathbf{y}_{i}, \boldsymbol{\theta}\right)}\right)\left(\mathbf{y}_{i}-\mathbf{X}_{i} \boldsymbol{\beta}\right) \mid \mathbf{V}_{i}, \mathbf{C}_{i}\right], \operatorname{com} d\left(\mathbf{y}_{i}, \boldsymbol{\theta}\right)=\left(\mathbf{y}_{i}-\mathbf{X}_{i} \boldsymbol{\beta}\right)^{\top} \boldsymbol{\Sigma}_{i}^{-1}\left(\mathbf{y}_{i}-\right.$ $\left.\mathbf{X}_{i} \boldsymbol{\beta}\right), \quad \mathbf{y}_{i} \sim T t_{n_{i}}\left(\mathbf{X}_{i} \boldsymbol{\beta}, \boldsymbol{\Sigma}_{i}, \nu ; \mathbb{A}_{i}\right), \mathbb{A}_{i}=\left\{\mathbf{y}_{i} \in \mathbb{R}^{n_{i}} \mid \mathbf{y}_{i} \leq \mathbf{a}_{i}\right\}, \mathbf{y}_{i}=\left(y_{i 1}, \ldots, y_{i n_{i}}\right)^{\top} \mathrm{e}$ $\mathbf{a}_{i}=\left(a_{i 1}, \ldots, a_{i n_{i}}\right)^{\top}$.

É importante ressaltar que $\mathbf{B}_{i}$ depende do cálculo de 


$$
\begin{aligned}
& {\widehat{u \mathbf{y}_{i}^{2}}}^{*}=E\left[\left(\frac{\nu+n_{i}}{\nu+d\left(\mathbf{y}_{i}, \boldsymbol{\theta}\right)}\right)^{2} \mathbf{y}_{i} \mathbf{y}_{i}^{\top} \mid \mathbf{V}_{i}, \mathbf{C}_{i}, \hat{\boldsymbol{\theta}}\right],{\widehat{u \mathbf{y}_{i}^{1}}}^{*}=E\left[\left(\frac{\nu+n_{i}}{\nu+d\left(\mathbf{y}_{i}, \boldsymbol{\theta}\right)}\right)^{2} \mathbf{y}_{i} \mid \mathbf{V}_{i}, \mathbf{C}_{i}, \hat{\boldsymbol{\theta}}\right] \\
& \widehat{\mathrm{u}}_{i}^{*}{ }^{*}=E\left[\left(\frac{\nu+n_{i}}{\nu+d\left(\mathbf{y}_{i}, \boldsymbol{\theta}\right)}\right)^{2} \mid \mathbf{V}_{i}, \mathbf{C}_{i}, \hat{\boldsymbol{\theta}}\right],
\end{aligned}
$$

assim, após algumas manipulações algébricas, temos três cenários possíveis:

- Se todas as observações do $i$-ésimo indivíduo forem censuradas, da Proposição 3:

$$
\begin{aligned}
& {\widehat{u \mathbf{y}_{i}^{2}}}^{*}=c_{n_{i}}(\nu, 2) \frac{T_{n_{i}}\left(\mathbf{V}_{i} \mid \widehat{\boldsymbol{\mu}}_{i}, \widehat{\boldsymbol{\Sigma}}_{i}^{*}, \nu+4\right)}{T_{n_{i}}\left(\mathbf{V}_{i} \mid \widehat{\boldsymbol{\mu}}_{i}, \widehat{\boldsymbol{\Sigma}}_{i}, \nu\right)} E\left[\mathbf{W}_{i} \mathbf{W}_{i}^{\top}\right], \\
& {\widehat{u \mathbf{y}_{i}^{1}}}^{*}=c_{n_{i}}(\nu, 2) \frac{T_{n_{i}}\left(\mathbf{V}_{i} \mid \widehat{\boldsymbol{\mu}}_{i}, \widehat{\boldsymbol{\Sigma}}_{i}^{*}, \nu+4\right)}{T_{n_{i}}\left(\mathbf{V}_{i} \mid \widehat{\boldsymbol{\mu}}_{i}, \widehat{\boldsymbol{\Sigma}}_{i}, \nu\right)} E\left[\mathbf{W}_{i}\right], \\
& {\widehat{u \mathbf{y}_{i}^{0}}}^{*}=c_{n_{i}}(\nu, 2) \frac{T_{n_{i}}\left(\mathbf{V}_{i} \mid \widehat{\boldsymbol{\mu}}_{i}, \widehat{\boldsymbol{\Sigma}}_{i}^{*}, \nu+4\right)}{T_{n_{i}}\left(\mathbf{V}_{i} \mid \widehat{\boldsymbol{\mu}}_{i}, \widehat{\boldsymbol{\Sigma}}_{i}, \nu\right)},
\end{aligned}
$$

em que $\mathbf{W}_{i} \sim T t_{n_{i}}\left(\widehat{\boldsymbol{\mu}}_{i}, \widehat{\boldsymbol{\Sigma}}_{i}, \nu+4, \mathbb{A}\right), \widehat{\boldsymbol{\mu}_{i}}=\mathbf{X}_{i} \widehat{\boldsymbol{\beta}}, \widehat{\boldsymbol{\Sigma}}_{i}^{*}=\frac{\nu}{\nu+4} \widehat{\boldsymbol{\Sigma}}_{i}$ and

$$
c_{n_{i}}(\nu, 2)=\left(\frac{\nu+n_{i}}{\nu}\right)^{2}\left[\frac{\Gamma\left(\frac{\nu+n_{i}}{2}\right) \Gamma\left(\frac{\nu+4}{2}\right)}{\Gamma\left(\frac{\nu}{2}\right) \Gamma\left(\frac{\nu+n_{i}+4}{2}\right)}\right] .
$$

- Se todas as observações do $i$-ésimo indivíduo forem não censuradas, então:

${\widehat{u \mathbf{y}_{i}^{2}}}^{*}=\left(\frac{\nu+n_{i}}{\nu+d\left(\mathbf{y}_{i}, \boldsymbol{\theta}\right)}\right)^{2} \mathbf{y}_{i} \mathbf{y}_{i}^{\top},{\widehat{u \mathbf{y}_{i}^{1}}}^{*}=\left(\frac{\nu+n_{i}}{\nu+d\left(\mathbf{y}_{i}, \boldsymbol{\theta}\right)}\right)^{2} \mathbf{y}_{i},{\widehat{u \mathbf{y}_{i}^{0}}}^{*}=\left(\frac{\nu+n_{i}}{\nu+d\left(\mathbf{y}_{i}, \boldsymbol{\theta}\right)}\right)^{2}$,

em que $d\left(\mathbf{y}_{i}, \boldsymbol{\theta}\right)=\left(\mathbf{y}_{i}-\mathbf{X}_{i} \boldsymbol{\beta}\right)^{\top} \boldsymbol{\Sigma}_{i}^{-1}\left(\mathbf{y}_{i}-\mathbf{X}_{i} \boldsymbol{\beta}\right)$.

- Se o $i$-ésimo indivíduo tem observações censuradas e não censuradas, então da Proposição 4 e dado que $\left\{\mathbf{y}_{i} \mid \mathbf{V}_{i}, \mathbf{C}_{i}\right\},\left\{\mathbf{y}_{i} \mid \mathbf{V}_{i}, \mathbf{C}_{i}, \mathbf{y}_{i}^{o}\right\}$, e $\left\{\mathbf{y}_{i}^{c} \mid \mathbf{V}_{i}, \mathbf{C}_{i}, \mathbf{y}_{i}^{o}\right\}$ são processos equivalentes, temos que:

$$
\begin{aligned}
& {\widehat{u \mathbf{y}_{i}^{2}}}^{*}=\left(\begin{array}{cc}
\widehat{u \mathbf{y}_{i}^{0}} \mathbf{y}_{i}^{o} \mathbf{y}_{i}^{o \top} & \widehat{u \mathbf{y}_{i}^{0}} \mathbf{y}_{i}^{o} \widehat{\mathbf{w}}_{i}^{\top} \\
\widehat{u \mathbf{y}_{i}^{0}} \widehat{\mathbf{w}_{i}^{c}} \mathbf{y}_{i}^{o \top} & \widehat{u \mathbf{y}_{i}^{0}} \widehat{\mathbf{w}_{i}^{c} \mathbf{w}_{i}^{c \top}}
\end{array}\right), \\
& {\widehat{u \mathbf{y}_{i}^{1}}}^{*}=\operatorname{vec}\left(\widehat{u \mathbf{y}_{i}^{0}} \mathbf{y}_{i}^{o}, \widehat{\mathbf{w}_{i}^{c}}\right) \\
& {\widehat{u \mathbf{y}_{i}^{0}}}^{*}=\left(\frac{d_{n_{i}}}{\left(\nu+d\left(\mathbf{y}_{i}^{o}, \boldsymbol{\theta}^{o}\right)\right)^{2}}\right) \frac{T_{n_{i}^{c}}\left(\mathbf{V}_{i} \mid \boldsymbol{\mu}_{i}^{c o}, \tilde{\mathbf{S}}_{i}^{c o}, \nu+n_{i}^{o}+4\right)}{T_{n_{i}^{c}}\left(\mathbf{V}_{i} \mid \boldsymbol{\mu}_{i}^{c o}, \boldsymbol{\Sigma}_{i}^{c o}, \nu+n_{i}^{o}\right)}, \\
& \text { em que } d_{n_{i}}=\left(\nu+n_{i}\right)^{2}\left(\frac{\Gamma\left(\frac{n_{i}+\nu}{2}\right) \Gamma\left(\frac{n_{i}^{o}+\nu+4}{2}\right)}{\Gamma\left(\frac{n_{i}^{o}+\nu}{2}\right) \Gamma\left(\frac{n_{i}+\nu+4}{2}\right)}\right), \quad \tilde{\mathbf{S}}_{i}^{c o}=\left(\frac{\nu+d\left(\mathbf{y}_{i}^{o}, \boldsymbol{\theta}^{o}\right)}{\nu+4+n_{i}^{o}}\right) \boldsymbol{\Sigma}_{i}^{c c . o} \\
& d\left(\mathbf{y}_{i}^{o}, \boldsymbol{\theta}^{o}\right)=\left(\mathbf{y}_{i}^{o}-\mathbf{X}_{i}^{o} \boldsymbol{\beta}\right)^{\top} \boldsymbol{\Sigma}_{i}^{o o-1}\left(\mathbf{y}_{i}^{o}-\mathbf{X}_{i}^{o} \boldsymbol{\beta}\right), \widehat{\mathbf{w}_{i}^{c}}=E\left[\mathbf{W}_{i}\right] \text { e } \widehat{\mathbf{w}_{i}^{c} \mathbf{w}_{i}^{c \top}}=E\left[\mathbf{W}_{i} \mathbf{W}_{i}^{\top}\right], \text { com } \\
& \mathbf{W}_{i} \sim T t_{n_{i}^{c}}\left(\boldsymbol{\mu}_{i}^{c o}, \tilde{\mathbf{S}}_{i}^{c o}, \nu+n_{i}^{o}+2, \mathbb{A}_{i}^{c}\right) \text { e } \boldsymbol{\mu}_{i}^{c o}, \boldsymbol{\Sigma}_{i}^{c c . o} \text { e } \mathbf{S}_{i}^{c o} \text { como definidos anteriormente. }
\end{aligned}
$$

Intervalos de confiança assintóticos e testes de hipóteses para os efeitos fixos também são obtidos assumindo que os estimadores de MV de $\boldsymbol{\beta}$, denotados por $\widehat{\boldsymbol{\beta}}$, têm aproximadamente uma distribuição $N_{p}\left(\boldsymbol{\beta}, \mathbf{J}_{\boldsymbol{\beta} \boldsymbol{\beta}}^{-1}\right)$. Na prática, $\mathbf{J}_{\boldsymbol{\beta} \boldsymbol{\beta}}$ é usualmente desconhecido e é necessário substituí-lo pela sua avaliação nas estimativas de MV, $\mathbf{J}_{\widehat{\boldsymbol{\beta}}} \widehat{\boldsymbol{\beta}}$. 


\subsection{Predição de valores futuros}

O problema relacionado com a previsão de valores futuros tem um grande impacto em muitas aplicações práticas. Nesse sentido, Rao (1987) apontou que a precisão das previsões de futuras observações podem ser tomadas também como uma medida alternativa de "qualidade de ajuste".

Com o intuito de propor uma estratégia para gerar predições das observações futuras sobre nosso modelo $t$-MLC, utilizaremos a proposta dada por Wang (2013) detalhada a seguir:

Seja $\mathbf{y}_{i, o b s}\left(n_{i, o b s} \times 1\right)$ o vetor de respostas observadas para um novo indivíduo $i$ até o $t$ ésimo tempo e seja $\mathbf{y}_{i, \text { pred }}\left(n_{i, \text { pred }} \times 1\right)$ o vetor de futuras respostas para o $(t+h)$-ésimo tempo, com $h=1,2, \ldots$ do mesmo indivíduo $i$ que se deseja prever, em que $\overline{\mathbf{X}}_{i}=\left(\mathbf{X}_{i, \text { obs }}, \mathbf{X}_{i, p r e d}\right)$ é a matriz de desenho de ordem $\left(n_{i, o b s}+n_{i, p r e d}\right) \times p$ associada a $\overline{\mathbf{y}}_{i}=\left(\mathbf{y}_{i, \text { obs }}^{\top}, \mathbf{y}_{i, p r e d}^{\top}\right)$, tal que $n_{i, o b s}$ e $n_{i, p r e d}$ representam o número de valores observados e o número de valores preditos para o indivíduo $i$, respectivamente.

Para contornar a presença de possíveis valores censurados existentes no vetor de respostas observadas $\mathbf{y}_{i, o b s}$, utilizaremos o procedimento de imputação via algoritmo ECM, apresentado na Subseção 5.4.1, e substituiremos esses valores "parcialmente observados" pelos valores imputados, com o intuito de obter o vetor de dados completamente observados, denotado por $\mathbf{y}_{i, o b s^{*}}=\left(\widetilde{\mathbf{y}}_{i}^{(c)}, \mathbf{y}_{i, o b s}\right)$.

A razão para usar o procedimento de imputação é que assim evitamos o cálculo da esperança condicional da distribuição t de Student truncada que aparece no passo E do algoritmo ECM devido à presença de censuras e é bastante complicada, o que pode gerar um custo computacional muito alto.

Assim, considerando os dados completamente observados $\mathbf{y}_{i, o b s^{*}}$ e os valores a serem previstos $\mathbf{y}_{i, p r e d}$, assumimos que

$$
\overline{\mathbf{y}}_{i}^{*}=\left(\mathbf{y}_{i, o b s^{*}}^{\top}, \mathbf{y}_{i, p r e d}^{\top}\right)^{\top} \sim t_{n_{i, o b s}+n_{i, p r e d}}\left(\mathbf{X}_{i} \boldsymbol{\beta}, \boldsymbol{\Sigma}_{i}, \nu\right)
$$

em que $\boldsymbol{\Sigma}_{i}$, definido em (5.3), pode ser representada por $\boldsymbol{\Sigma}_{i}=\left(\begin{array}{cc}\boldsymbol{\Sigma}_{i}^{\text {obs*,obs* }} & \boldsymbol{\Sigma}_{i}^{\text {obs*,pred }} \\ \boldsymbol{\Sigma}_{i}^{\text {pred,obs* }} & \boldsymbol{\Sigma}_{i}^{\text {pred,pred }}\end{array}\right)$.

Como mencionado em Wang (2013) e Rao (1973), o melhor estimador linear de $\mathbf{y}_{i, \text { pred }}$ com respeito ao critério do erro quadrático médio (EQM) é a média condicional de $\mathbf{y}_{i, p r e d}$ dado $\mathbf{y}_{i, o b s^{*}}$, definida por

$$
\widehat{\mathbf{y}}_{i, p r e d}=\mathbf{X}_{i, p r e d} \boldsymbol{\beta}+\boldsymbol{\Sigma}_{i}^{p r e d, o b s^{*}} \boldsymbol{\Sigma}_{i}^{o b s^{*}, o b s^{*}-1}\left(\mathbf{y}_{i, o b s^{*}}-\mathbf{X}_{i, o b s^{*}} \boldsymbol{\beta}\right) .
$$

Portanto, o estimador de $\mathbf{y}_{i, \text { pred }}$ pode ser calculado diretamente substituindo $\widehat{\boldsymbol{\theta}} \mathrm{em}$ (5.12), o que leva a

$$
\widehat{\mathbf{y}}_{i, p r e d}=\widehat{\mathbf{y}}_{i, p r e d}(\widehat{\boldsymbol{\theta}})
$$




\subsection{Estudos de simulação}

Com o intuito de estudar o desempenho do nosso modelo proposto, apresentamos dois estudos de simulação. A primeira parte deste estudo avalia o desempenho do procedimento de imputação de observações censuradas no modelo $t$-MLC, considerando dois casos particulares da estrutura de correlação DEC: a estrutura UNC, que ocorre quando $\mathbf{E}_{i}=\mathbf{I}_{n i}$ e a estrutura U, que ocorre quando os parâmetros $\phi_{1}$ e $\phi_{2}$ da matriz $\mathbf{E}_{i}$ são desconhecidos, e é necessário estima-los.

O segundo estudo de simulação mostra que os estimadores dos parâmetros, com base no algoritmo ECM, para o modelo $t$-MLC possuem boas propriedades assintóticas. Para ambos os esquemas de simulação, consideraremos o modelo $t$-MLC definido na Subseção 5.3.2. Os parâmetros utilizados no modelo são: $\beta_{1}=2,5$ e $\beta_{2}=4$, como coeficientes de regressão, $\sigma^{2}=4$ como parâmetro de escala e $\phi_{1}=0,8$ e $\phi_{2}=1$ para a estrutura de correlação. Neste caso, os tempos de avaliação são dados por $\mathbf{t}_{i}=(1,3,5,7,10,14)^{\top}$, para todo $i=1, \ldots, n \mathrm{e}$ $\mathbf{X}_{i}=\mathbf{t}_{i}$.

\subsubsection{Simulação 1: Imputação das observações censuradas}

Como mencionado inicialmente, o objetivo deste estudo de simulação é comparar o desempenho do processo de imputação de observações censuradas no modelo $t$-MLC, sob dois tipos de estrutura de correlação DEC: a estrutura U versus a estrutura UNC . Para este propósito, procedemos da seguinte forma,

1. geramos $M=100$ conjuntos de dados de tamanho $n=300$ do modelo $t$-MLC com uma estrutura de correlação DEC, com $\mathbf{E}_{i}=0,8^{\left|t_{i j}-t_{i k}\right|}$, considerando quatro níveis de censura: $\gamma=5 \%, 15 \%, 25 \%$ e $35 \%$. É importante notar que, o objetivo aquí é estudar o efeito dos níveis de censuras na estimação considerando uma estrutura de correlação incorreta;

2. todas as observações censuradas foram imputadas utilizando o mecanismo descrito na Subseção 5.4.1 considerando as estruturas de correlação U e UNC.

Com o objetivo de comparar o desempenho das estruturas de correlação U e UNC, através do EM-imputação definido na Seção 5.4.1, utilizamos duas medidas de discrepância empírica utilizados por Wang \& Fan (2010) e Wang (2013): o erro médio absoluto (MAE) e o erro quadrático médio (EQM), definidos por:

$$
\mathrm{MAE}=\frac{1}{k} \sum_{i, j}\left|y_{i j}-\widetilde{y}_{i j}\right| \quad \text { e } \quad \mathrm{EQM}=\frac{1}{k} \sum_{i, j}\left(y_{i j}-\widetilde{y}_{i j}\right)^{2},
$$

em que $y_{i j}$ é o valor original simulado (antes de ser considerado como observação censurada) e $\widetilde{y}_{i j}$ é o valor imputado através do algoritmo EM, para $i=1, \ldots, 300$ e $j=1, \ldots, 6$. Note que, para $\gamma=5 \%$ temos que $k=90$, para $\gamma=15 \% \quad k=270$, para $\gamma=25 \% \quad k=450$ e para $\gamma=35 \% \quad k=630$. 


\begin{tabular}{cccccc}
\hline & \multicolumn{3}{c}{ Estrutura de Correlação } \\
\cline { 2 - 3 } \cline { 5 - 6 } Nível de & \multicolumn{2}{c}{ U } & & \multicolumn{2}{c}{ UNC } \\
\cline { 2 - 3 } \cline { 5 - 6 } censura & MAE & EQM & & MAE & EQM \\
\hline $\mathbf{5 \%}$ & 1,120052 & 2,744973 & & 1,199131 & 3,075949 \\
$\mathbf{1 5 \%}$ & 1,293753 & 3,106423 & & 1,563213 & 4,340442 \\
$\mathbf{2 5 \%}$ & 1,409025 & 3,902546 & & 1,684068 & 5,475168 \\
$\mathbf{3 5 \%}$ & 1,568360 & 4,647703 & & 1,830202 & 6,170776 \\
\hline
\end{tabular}

Tabela 5.2: Simulação 1. Média do MAE e do EQM sobre os $M=100$ conjunto de dados. UNC: Sem estrutura de correlação e U: Parâmetros $\phi_{1}$ e $\phi_{2}$ da matriz $\mathbf{E}_{i}$ desconhecidos.

A média do MAE e do EQM, tomada dentre os 100 conjuntos de dados são apresentadas na Tabela 5.2 e Figura 5.2. Observamos que, em todos os casos, a estrutura de correlação U apresenta valores imputados com menor viés do que a estrutura UNC.

(a)

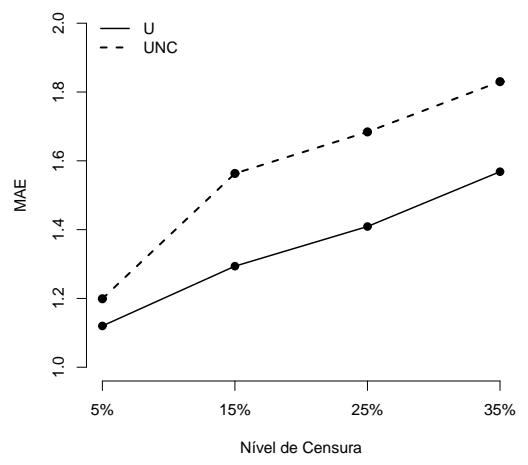

(b)

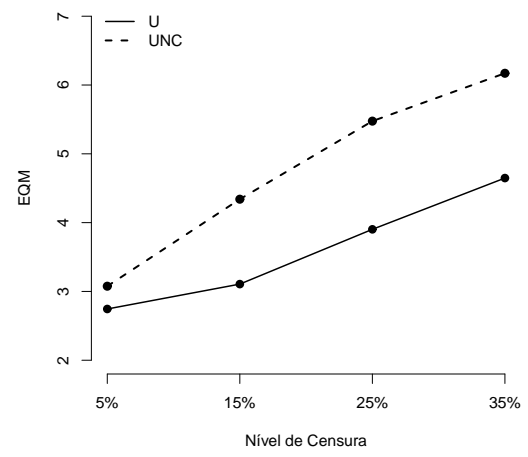

Figura 5.2: Simulação 1. Média do (a) MAE e (b) EQM sobre $M=100$ conjuntos de dados do modelo $t$-MLC com estrutura de correlação U e UNC.

\subsubsection{Simulação 2: Propriedades Assintóticas}

Neste estudo, analisamos o viés absoluto (Viés) e o erro quadrático médio (EQM) dos coeficientes de regressão estimados para o modelo $t$-MLC considerando 6 diferentes tamanhos de amostras $(n=50,100,200,300,400$ e 600). Estas medidas são definidas por:

$$
\text { Viés }\left(\theta_{i}\right)=\frac{1}{M} \sum_{j=1}^{M}\left|\widehat{\theta}_{i}^{(j)}-\theta_{i}\right| \quad \text { e } \operatorname{EQM}\left(\theta_{i}\right)=\frac{1}{M} \sum_{j=1}^{M}\left(\widehat{\theta}_{i}^{(j)}-\theta_{i}\right)^{2},
$$

em que $\widehat{\theta}_{i}^{(j)}$ é estimador de MV do parâmetro $\theta_{i}$ para a $j$-ésima amostra.

O intuito desta simulação é mostrar, através de evidências empíricas, a convergência dos estimadores de MV do modelo $t$-MLC, considerando uma estrutura de correlação DEC.

Para cada tamanho de amostra foi gerado $M=100$ conjuntos de dados, considerando um nível de censura de $5 \%$. Neste esquema de simulação o parâmetro $\phi_{2}$ é igual a 1 , o que 
reduz o modelo geral ao modelo autoregressivo de tempo contínuo de ordem 1, denotado por AR(1). Utilizando o algoritmo ECM proposto, o valor médio do Viés e do EQM para cada parâmetro sobre os 100 conjuntos de dados foram calculados.

A Figura 5.3 mostra que o Viés absoluto e o EQM médio convergem para zero quando o tamanho da amostra " $n$ " aumenta. Como era esperado, o algoritmo ECM proporciona estimadores com boas propriedades assintóticas, mesmo quando as observações da variável resposta são censuradas e/ou correlacionadas.
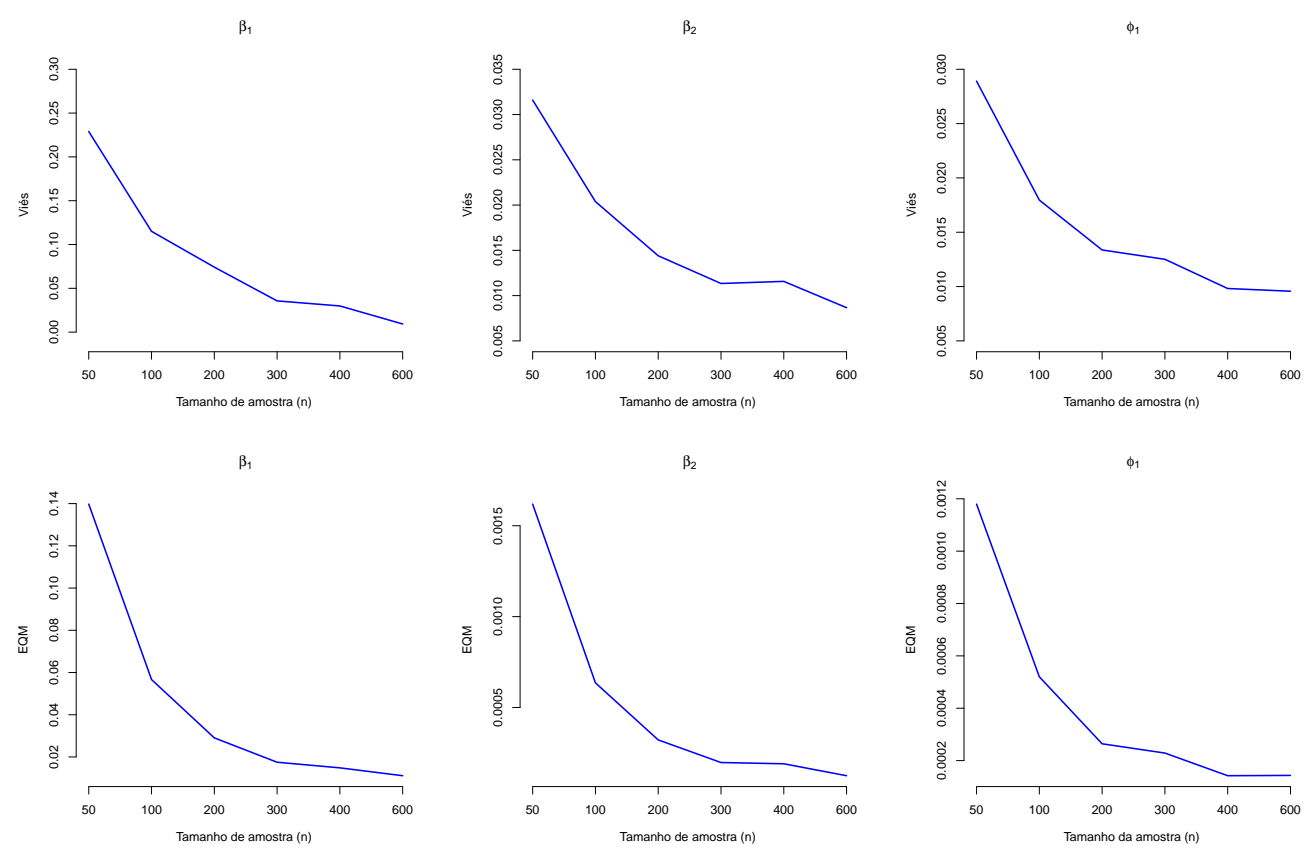

Figura 5.3: Simulação 2. Viés (1 $1^{\text {ra }}$ linha) e EQM (2 $2^{\text {da }}$ linha) dos parâmetros estimados no modelo $t$-MLC com $5 \%$ de censura.

\subsection{Aplicação}

Nesta seção aplicaremos as técnicas propostas para o conjunto de dados UTI, descrito na Seção 5.2 . Este conjunto de dados consiste em 362 observações, das quais 26 estavam abaixo dos limites de detecção considerados (50 ou 400 copies $/ m L$ ), isto é, temos 26 obsevações censuradas.

Como foi mencionado anteriormente, os dados UTI foram analisados previamente por Lachos et al. (2011), indicando que as inferências baseadas na suposição do modelo "normal" eram questionáveis. Desta forma, reavaliamos este conjunto de dados com o intuito de realizar um ajuste mais robusto, considerando agora um modelo de regressão correlacionado a partir de uma perspectiva frequentista. Para isso, consideraremos o modelo $t$-MLC com uma estrutura de correlação $\mathbf{E}_{i}$ definida na Seção 5.3. Neste caso, $y_{i j}$ é o $\log _{10} \mathrm{HIV}-1$ para o $i$-ésimo indivíduo no tempo $t_{j}$, com $t_{1}=0, t_{2}=1, t_{3}=3, t_{4}=6, t_{5}=9, t_{6}=12, t_{7}=18$, e $t_{8}=24$. 
Consideraremos quatro casos de estrutura de correlação induzidos pela especificação da matriz $\mathbf{E}_{i}$ : (a) a estrutura UNC, (b) o caso de tempos contínuos $\mathrm{AR}(1)$, (c) o caso MA(1) e (d) o caso não especificado (U), em que $\phi_{1}$ e $\phi_{2}$ são desconhecidos, com $0 \leq \phi_{1}<1$, e $\phi_{2} \geq 0$.

O grau de liberdade $\nu$ foi suposto conhecido e fixado como a parte inteira do valor que maximiza a log-verossimilhança do modelo $t$-MLC. A Figura 5.4 mostra que a função de log-verossimilhança atinge o valor máximo quando $\nu=10$. As estimativas de MV dos demais parâmetros foram obtidas utilizando o algoritmo ECM, desenvolvido na Seção 5.4. Os valores iniciais foram obtidos como foi sugerido no pacote lmec (Vaida \& Liu, 2012).

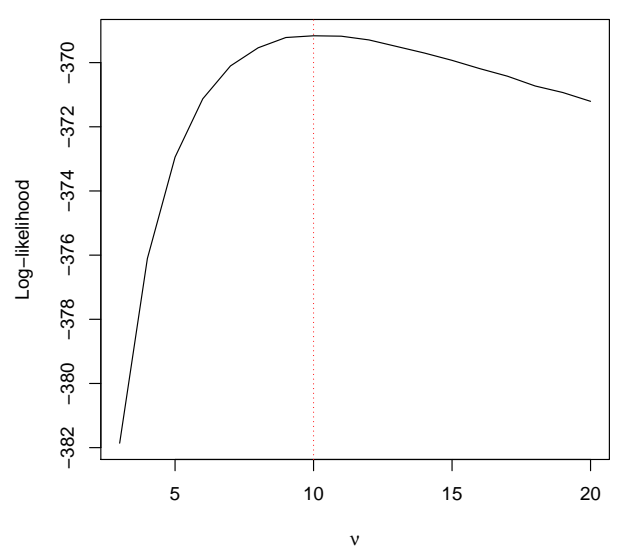

Figura 5.4: Dados UTI. Log-verossimilhança perfilada dos graus de liberdade $\nu$.

A Tabela 5.3 apresenta as estimativas de MV e o erro padrão dos parâmetros de regressão $\boldsymbol{\beta}$ para os modelos lineares censurados $t$ de Student e normal multivariado. Embora as estimativas sejam bastante semelhantes em ambos os casos, os erros padrões são menores para o caso do modelo $t$ de Student. Na aplicação foram considerados oito modelos como resultado da combinação das quatro estruturas de correlação (UNC, AR(1), MA(1) e U) com as duas distribuições consideradas (normal e $t$ de Student).

O valor da função de log-verossimilhança e dos critérios de seleção de modelos, tais como AIC e BIC, são apresentados na Tabela 5.4. É importante ressaltar que todos esses critérios indicam que os modelos que consideram a distribuição $t$ de Student multivariada apresentam melhores ajustes do que aqueles que consideram a distribuição normal (para uma mesma estrutura de correlação). Além disso, os critérios indicam uma preferência pela estrutura de correlação não especificada $(\mathrm{U})$, obtida quando os parâmetros da matriz $\mathbf{E}_{i}$ são calculados a partir dos dados.

Os coeficientes de regressão $\beta_{j}$, para $j=1, \ldots, 8$, em geral aumentam gradualmente no tempo, qualquer que seja a estrutura de correlação e a distribuição assumida (salvo $\beta_{5} \mathrm{em}$ alguns casos). Isto mostra o efeito negativo da interrupção da terapia anti-retroviral.

Para o modelo ( $t$-MLC) com estrutura de correlação (U), os coeficientes aumentam 


\begin{tabular}{|c|c|c|c|c|c|c|c|c|}
\hline \multirow{3}{*}{ Parâmetros } & \multicolumn{8}{|c|}{ N-MLC } \\
\hline & \multicolumn{2}{|c|}{ UNC } & \multicolumn{2}{|c|}{$\operatorname{AR}(1)$} & \multicolumn{2}{|c|}{ MA(1) } & \multicolumn{2}{|c|}{$\mathbf{U}$} \\
\hline & Est & $\mathrm{EP}$ & Est & $\mathrm{EP}$ & Est & $\mathrm{EP}$ & Est & $\mathrm{EP}$ \\
\hline$\beta_{1}$ & 3,6160 & 0,0153 & 3,6334 & 0,0162 & 3,6194 & 0,0150 & 3,6196 & 0,0156 \\
\hline$\beta_{2}$ & 4,1527 & 0,0172 & 4,2095 & 0,0168 & 4,1825 & 0,0166 & 4,1834 & 0,0164 \\
\hline$\beta_{3}$ & 4,2381 & 0,0184 & 4,2502 & 0,0182 & 4,2384 & 0,0181 & 4,2568 & 0,0169 \\
\hline$\beta_{4}$ & 4,3727 & 0,0187 & 4,3224 & 0,0189 & 4,3729 & 0,0184 & 4,3738 & 0,0170 \\
\hline$\beta_{5}$ & 4,3650 & 0,0248 & 4,4680 & 0,0237 & 4,3652 & 0,0245 & 4,5791 & 0,0195 \\
\hline$\beta_{6}$ & 4,2326 & 0,0313 & 4,3781 & 0,0303 & 4,2327 & 0,0309 & 4,5819 & 0,0221 \\
\hline$\beta_{7}$ & 4,3258 & 0,0444 & 4,3749 & 0,0463 & 4,3260 & 0,0438 & 4,6879 & 0,0275 \\
\hline$\beta_{8}$ & 4,5620 & 0,0818 & 4,5762 & 0,0842 & 4,5620 & 0,0807 & 4,8061 & 0,0418 \\
\hline$\sigma^{2}$ & 1,0631 & & 1,1498 & & 1,0486 & & 1,1053 & \\
\hline$\phi_{1}$ & - & & 0,8251 & & 0,4068 & & 0,7027 & \\
\hline$\phi_{2}$ & - & & 1,00 & & $\infty$ & & 0,0286 & \\
\hline \multirow{3}{*}{ Parâmetros } & \multicolumn{8}{|c|}{$t$-MLC } \\
\hline & \multicolumn{2}{|c|}{ UNC } & \multicolumn{2}{|c|}{$\operatorname{AR}(1)$} & \multicolumn{2}{|c|}{ MA(1) } & \multicolumn{2}{|c|}{$\mathbf{U}$} \\
\hline & Est & $\mathrm{EP}$ & Est & $\mathrm{EP}$ & Est & $\mathrm{EP}$ & Est & $\mathrm{EP}$ \\
\hline$\beta_{1}$ & 3,6511 & 0,0120 & 3,6410 & 0,0155 & 3,6578 & 0,0120 & 3,6330 & 0,0153 \\
\hline$\beta_{2}$ & 4,2386 & 0,0146 & 4,3022 & 0,0172 & 4,2706 & 0,0144 & 4,2697 & 0,0171 \\
\hline$\beta_{3}$ & 4,3149 & 0,0156 & 4,3312 & 0,0187 & 4,3246 & 0,0156 & 4,3290 & 0,0177 \\
\hline$\beta_{4}$ & 4,4715 & 0,0159 & 4,4297 & 0,0195 & 4,4792 & 0,0159 & 4,4715 & 0,0178 \\
\hline$\beta_{5}$ & 4,5268 & 0,0210 & 4,5476 & 0,0248 & 4,5293 & 0,0209 & 4,6359 & 0,0206 \\
\hline$\beta_{6}$ & 4,3923 & 0,0267 & 4,4435 & 0,0317 & 4,3963 & 0,0266 & 4,6238 & 0,0235 \\
\hline$\beta_{7}$ & 4,5012 & 0,0373 & 4,4660 & 0,0475 & 4,5092 & 0,0377 & 4,7082 & 0,0295 \\
\hline$\beta_{8}$ & 4,6896 & 0,0692 & 4,6481 & 0,0863 & 4,5092 & 0,0687 & 4,7998 & 0,0455 \\
\hline$\sigma^{2}$ & 0,8092 & & 1,0272 & & 0,8003 & & 1,0103 & \\
\hline$\phi_{1}$ & - & & 0,7754 & & 0,2752 & & 0,6629 & \\
\hline$\phi_{2}$ & - & & 1,00 & & $\infty$ & & 0,0222 & \\
\hline$\nu$ & 10,00 & & 10,00 & & 10,00 & & 10,00 & - \\
\hline
\end{tabular}

Tabela 5.3: Dados UTI. Estimativas de MV e erro padrão (EP) para os coeficientes de regressão sob os modelos MLC normal e $t$ com estrutura de correlação DEC. UNC: Sem estrutura de correlação e U: Parâmetros $\phi_{1}$ e $\phi_{2}$ da matriz $\mathbf{E}_{i}$ desconhecidos.

de 3.63, no começo do estudo, para 4.79 no final do mesmo. Note que, considerando um intervalo assintótico de $95 \%$, todos os coeficientes de regressão são significativos. A estimação do parâmetro de escala $\sigma^{2}$ entre os indivíduos na escala $\log _{10}$ é de 1.01 .

Sabe-se que a presença de observações discrepantes pode afetar a estimação de MV dos parâmetros sob a suposição de normalidade. Assim, no contexto dos modelos censurados de efeitos mistos, Matos et al. (2013) analisaram os dados UTI e detectaram que os indivíduos $\sharp 20, \sharp 35, \sharp 41$ e $\sharp 42$ são considerados como influentes. Estes resultados coincidem com os obtidos por Lachos et al. (2011), sob uma perspectiva Bayesiana. Ao avaliar este conjunto de dados considerando o modelo $t$-MLC com estrutura de correlação DEC, notamos que, como era esperado, este modelo pondera a influência destes casos discrepantes nas estimativas de MV, atribuindo-lhes pesos pequenos no procedimento de estimação. Assim, os pesos estimados $\left(\widehat{u}_{i}, i=1, \ldots, 72\right)$ para o modelo $t$-MLC são apresentados na Figura 5.5. Através 


\begin{tabular}{|c|c|c|c|c|c|c|c|c|}
\hline \multirow[b]{2}{*}{ Critérios } & \multicolumn{4}{|c|}{ N-MLC } & \multicolumn{3}{|c|}{$t$-MLC } & \multirow[b]{2}{*}{$\mathbf{U}$} \\
\hline & UNC & $\operatorname{AR}(1)$ & MA(1) & $\mathbf{U}$ & UNC & $\operatorname{AR}(1)$ & MA(1) & \\
\hline log-verossim & $-524,166$ & $-463,043$ & $-516,507$ & $-411,926$ & $-484,165$ & $-421,249$ & $-476,647$ & $-369,129$ \\
\hline AIC & 1066,333 & 946,087 & 1053,014 & 845,852 & 986,331 & 862,498 & 973,295 & 760,259 \\
\hline $\mathrm{BIC}$ & 1101,357 & 985,004 & 1091,931 & 888,660 & 1021,357 & 901,415 & 1012,212 & 803,067 \\
\hline$A I C_{\text {corr }}$ & 1066,844 & 946,714 & 1053,641 & 846,607 & 986,843 & 863,125 & 973,922 & 761,014 \\
\hline
\end{tabular}

Tabela 5.4: Dados UTI. Comparação entre os modelos MLC normal e $t$ de Student. UNC: Sem estrutura de correlação e U: Parâmetros $\phi_{1}$ e $\phi_{2}$ da matriz $\mathbf{E}_{i}$ desconhecidos.

desta figura notamos que os indivíduos $\sharp 20, \sharp 35$, $\sharp 41$ e $\sharp 42$ apresentam pesos pequenos, verificando a robustez no procedimento para encontrar as estimativas de MV sob a suposição da distribuição $t$ de Student.

(a)

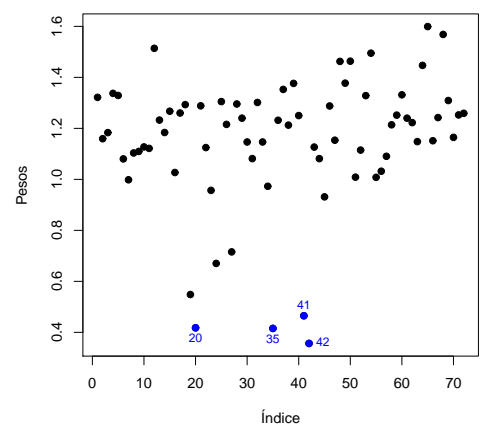

(b)

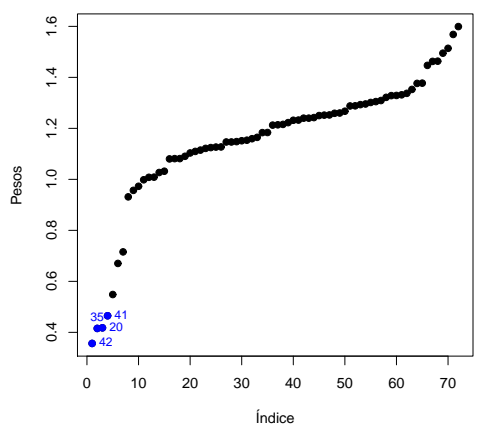

(c)

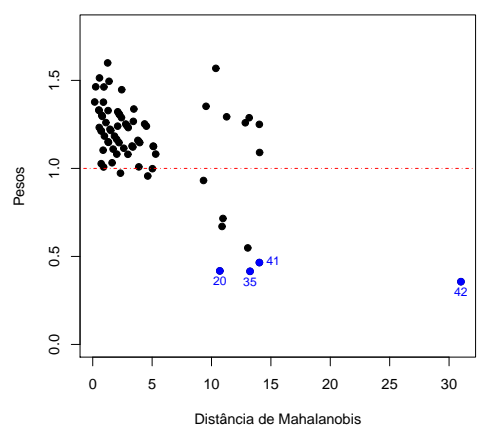

Figura 5.5: Dados UTI. Pesos estimados $\widehat{u}_{i}$ para o modelo $t$-MLC.

Por outro lado, seguindo o que foi desenvolvido por Wang \& Fan (2010) e Wang (2013), avaliaremos a predição de valores futuros utilizando nossa proposta apresentada na Seção 5.6 .

Assim, como uma simples ilustração, selecionamos os indivíduos que foram avaliados pelo menos seis vezes (29 casos no total) com o intuito de prever as duas últimas medições para cada um deles. A Tabela 5.5 mostra a comparação entre os valores preditos (um e dois passos ao frente) com os valores reais, sob o modelo $t$-MLC, considerando três diferentes casos de estrutura DEC: AR(1), MA(1) e U. Como considerado no estudo de simulação, apresentado na Seção 5.7, utilizaremos as medidas MAE e EQM para comparar a precisão na predição, sob as três diferentes estruturas DEC consideradas.

Observamos que a estrutura de correlação U apresenta melhores resultados em comparação a todas as outras do ponto de vista de preditivo. 


\begin{tabular}{|c|c|c|c|c|c|c|}
\hline \multirow{3}{*}{$\begin{array}{c}\sharp \text { de } \\
\text { Predições }\end{array}$} & \multicolumn{6}{|c|}{$t$-MLC } \\
\hline & \multicolumn{2}{|c|}{$\mathbf{U}$} & \multicolumn{2}{|c|}{$\operatorname{AR}(1)$} & \multicolumn{2}{|c|}{ MA(1) } \\
\hline & MAE & EQM & MAE & EQM & MAE & EQM \\
\hline uma observação & 0,330835 & 0,191284 & 0,438885 & 0,270230 & 0,623717 & 0,519735 \\
\hline duas observações & 0,372141 & 0,215979 & 0,522241 & 0,504930 & 0,641799 & 0,702774 \\
\hline
\end{tabular}

Tabela 5.5: Dados UTI. Avaliação da precisão das predições considerando o modelo $t$ MLC com diferentes estruturas de correlação DEC. U: Parâmetros $\phi_{1}$ e $\phi_{2}$ da estrutura de corelação desconhecidos.

A Figura 5.6 mostra a comparação entre os dados reais com os valores preditos, dois passos a frente, sob três estruturas de correlação DEC: AR(1), MA(1) e U para os indivíduos $\sharp 4, \sharp 15, \sharp 61$. Podemos ver a partir destes resultados, como a estrutura U supera as outras estruturas de correlação desde um ponto de vista preditivo, isto é, a estrutura U gera valores preditivos mais próximos aos reais.
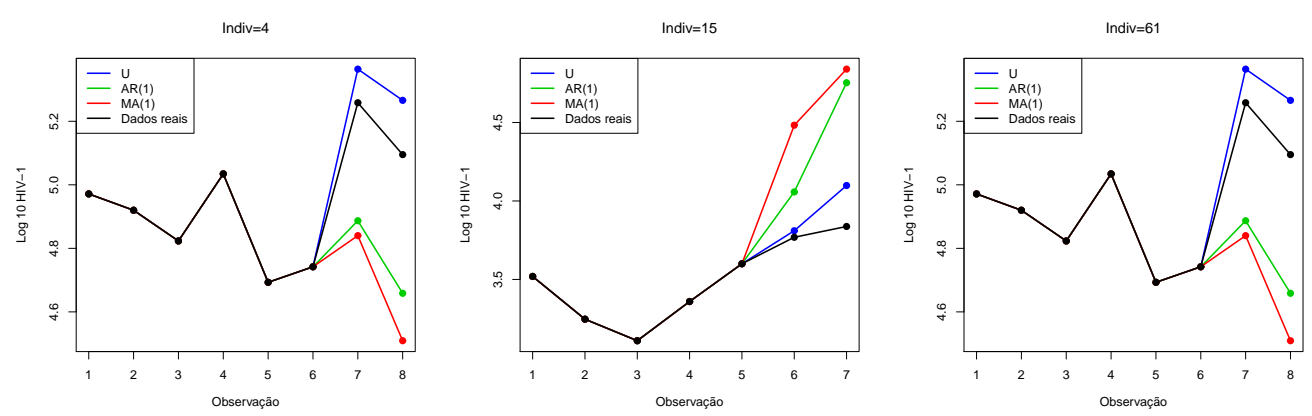

Figura 5.6: Dados UTI. Evaluação da performance da predição para três indivíduos escolhidos aleatóriamente. 


\section{Capítulo 6}

\section{Conclusões Finais e Perspectivas}

\section{Futuras}

\subsection{Conclusões Finais}

Nesta tese, apresentamos uma abordagem clássica e Bayesiana dos modelos lineares censurados sob a classe de distribuições SMN, denotados por SMN-CR. Esta é uma generalização dos trabalhos apresentados por Barros et al. (2010), Arellano-Valle et al. (2012) e Massuia et al. (2012). A classe de distribuições SMN oferece um alto grau de flexibilidade para trabalhar com dados censurados com a presença de outliers, e tem como casos especiais distribuições como a Normal, t de Student, slash, normal contaminada, entre outras.

A partir de uma perspectiva clássica, foi desenvolvido um algoritmo tipo EM para calcular estimativas de máxima verossimilhança dos modelos. Esta metodologia foi aplicada e testada em um conjunto de dados reais (Salários), assim como em dados simulados com o intuito de mostrar como nosso procedimento pode ser utilizado para avaliar modelos censurados, identificar outliers e obter estimativas robustas para os parmâmetros.

Sob a perspectiva Bayesiana, apresentamos um algoritmo tipo Gibbs, com um passo Metropolis-Hasting para determinar as estimativas Bayesianas dos modelos SMN-CR, implementando também algumas medidas de divergência e de influência Bayesiana baseadas no método de eliminação de casos, as quais foram testadas no conjunto de dados reais Salários.

A partir dos resultados, motrou-se que os modelos SMN-CR apresentaram melhores estimativas do que o normal na presença de valores atípicos, uma vez que possuem caudas mais pesadas.

É importante ressaltar que técnicas foram codificadas e implementadas em dois pacotes para o R: SMNCensReg e BayesCR, os quais podem ser livremente instalados e são constantemente atualizados para melhor utilização por parte dos usuários.

Propusemos uma abordagem robusta para modelos lineares de respostas censuradas e correlacionadas com base na distribuição t de Student multivariada, denotada por t-LMC, considerando uma estrutura de correlação (DEC) para a matriz e variância e covariância 
dos erros aleatórios. Este modelo oferece grande flexibilidade ao manipular dados longitudinais na presença de outliers. A metodologia foi aplicada ao conjunto de dados de HIV, considerado por Vaida \& Liu (2009) e Matos et al. (2013).

\subsection{Perspectivas Futuras}

Vários trabalhos de pesquisa podem ser derivados e/ou direcionados a partir dos resultados deste trabalho, entre eles podemos mencionar os seguintes:

- Estender os resultados de estimação obtidos nos modelos SMN-CR para a classe de distribuições misturas da escala de skew normal (SMSN) definida por Branco \& Dey (2001).

- Estender os resultados de estimação e a análise de diagnóstico, encontrados nos modelos t-MLC com estrutura de correlação para $\Sigma_{i}$, para a classe de Misturas da escala da normal (MSN) multivariada apresentada no Capítulo 1

- Estender o trabalho de modelos de misturas finitas sob a classe assimétrica, desenvolvido por Basso et al. (2010) e Lee \& Scott (2012) para o caso dos modelos com a presença de censuras.

- Realizar um estudo de inferência e diagnóstico nos modelos com erro de medidas para respostas censuradas, sob a classe SMN e SMSN, tanto sob uma perspectiva clássica quanto Bayesiana.

- Os métodos propostos nesta tese, também podem ser aplicados e estendidos para o contexto dos Modelos de efeitos aleatórios. 


\section{Apêndice A}

\section{Lemas e Corolários}

Os seguintes Lemas, desenvolvidos por Kim (2008) e Genç (2013) são úteis para avaliar algumas integrais utilizadas neste trabalho na implementação do nosso algoritmo tipo EM

Lema 1. Se $Z \sim T N_{(a, b)}(0,1)$, então

$$
(k+1) E\left[Z^{k}\right]-E\left[Z^{k+2}\right]=\frac{(b)^{k+1} \phi(b)-(a)^{k+1} \phi(a)}{\Phi(b)-\Phi(a)},
$$

para $k=-1,0,1,2, \ldots$

Demonstração. Veja Lema 2.3 em Kim (2008).

Lema 2. Seja U uma variável aletória aleatória positiva. Então $F_{S M N}(a)=E_{U}\left[\Phi\left(a U^{\frac{1}{2}}\right)\right]$, em que $F_{S M N}(\cdot)$ denota a fda da variável aleatória SMN padronizada, isto é, quando $\mu=0$ $e \sigma^{2}=1$.

Demonstração. Veja Lema 3 em Genç (2013).

Lema 3. Para $\nu>0$,

$$
\int_{0}^{u} x^{v-1} e^{-\mu x} d x=\mu^{-v} \gamma^{*}(v, \mu u)
$$

em que $\gamma^{*}(a, x)=\int_{0}^{x} e^{-t} t^{a-1} d t$ é a função gama incompleta.

Demonstração. Veja Lema 6 em Genç (2013).

Os seguintes Corolários são uma direta consequência da Proposição 1 dada na Seção 1.1. As demonstrações dos Corolários 2 e 3 seguem diretamente da definição da variância e da representação estocástica de uma variável aleatória SMN, dada na equação (1.1).

Corolário 1. Seja $Y \sim \operatorname{SMN}\left(\mu, \sigma^{2}, \nu\right)$ com fator de escala $U$ e $\mathcal{A}=(a, b)$. Então, para $r \geq 1$

$$
\begin{aligned}
\mathrm{E}\left[U^{r} \mid Y \in \mathcal{A}\right] & =\mathrm{E}\left[U^{r} \mid X \in \mathcal{A}^{*}\right] \\
\mathrm{E}\left[U^{r} Y \mid Y \in \mathcal{A}\right] & =\mu \mathrm{E}\left[U^{r} \mid X \in \mathcal{A}^{*}\right]+\sigma \mathrm{E}\left[U^{r} X \mid X \in \mathcal{A}^{*}\right] ; \\
\mathrm{E}\left[U^{r} Y^{2} \mid Y \in \mathcal{A}\right] & =\mu^{2} \mathrm{E}\left[U^{r} \mid X \in \mathcal{A}^{*}\right]+2 \mu \sigma \mathrm{E}\left[U^{r} X \mid X \in \mathcal{A}^{*}\right]+\sigma^{2} \mathrm{E}\left[U^{r} X^{2} \mid X \in \mathcal{A}^{*}\right],
\end{aligned}
$$

em que $X \sim \operatorname{SMN}(0,1, \boldsymbol{\nu})$ e $\mathcal{A}^{*}=\left(a^{*}, b^{*}\right)$, com $a^{*}=(a-\mu) / \sigma$ e $b^{*}=(b-\mu) / \sigma$. 
Corolário 2. Seja $X \sim \operatorname{SMN}(0,1, \boldsymbol{\nu})$ com fator de escala $U$ e $\mathcal{A}=(a, b)$. Então, para $r \geq 1$

$$
\begin{aligned}
\operatorname{Var}\left[U^{r} \mid X \in \mathcal{A}\right] & =\tau(a, b)\left[\mathrm{E}_{\Phi}(2 r, b)-\mathrm{E}_{\Phi}(2 r, a)-\tau(a, b)\left\{\mathrm{E}_{\Phi}(r, b)-\mathrm{E}_{\Phi}(r, a)\right\}^{2}\right] \\
\operatorname{Var}\left[U^{r} X \mid X \in \mathcal{A}\right] & =\tau(a, b)\left[\mathrm{E}_{\Phi}(2 r-1, b)-\mathrm{E}_{\Phi}(2 r-1, a)\right. \\
& \left.+a b \mathrm{E}_{\phi}\left(2 r-\frac{1}{2}, a\right) \mathrm{E}_{\phi}\left(2 r-\frac{1}{2}, b\right)-\tau(a, b)\left\{\mathrm{E}_{\Phi}(r, b)-\mathrm{E}_{\Phi}(r, a)\right\}^{2}\right],
\end{aligned}
$$

em que $\tau(a, b)$ é dada em (1.5).

Corolário 3. Seja $X \sim \operatorname{SMN}(0,1, \boldsymbol{\nu})$ com fator de escala $U, \mathcal{A}=(a, b)$ e $Y=\mu+\sigma X$. Então, para $r \geq 1$,

$$
\begin{aligned}
\operatorname{Var}\left[U^{r} \mid Y \in \mathcal{A}\right]= & \operatorname{Var}\left[U^{r} \mid X \in \mathcal{A}^{*}\right] \\
\operatorname{Var}\left[U^{r} Y \mid Y \in \mathcal{A}\right]= & \mu^{2} \operatorname{Var}\left[U^{r} \mid X \in \mathcal{A}^{*}\right]+\sigma^{2} \operatorname{Var}\left[U^{r} X \mid X \in \mathcal{A}^{*}\right] \\
& +2 \mu \sigma \operatorname{Cov}\left[U^{r}, U^{r} X \mid X \in \mathcal{A}^{*}\right]
\end{aligned}
$$

em que $\mathcal{A}^{*}=\left(a^{*}, b^{*}\right)$, com $a^{*}=(a-\mu) / \sigma e b^{*}=(b-\mu) / \sigma$. 


\section{Apêndice B}

\section{Cálculo de $\boldsymbol{E}_{\phi}(r, h)$ e $\boldsymbol{E}_{\Phi}(r, h)$}

Neste apêndice são calculados e apresentados os valores esperados $\mathrm{E}_{\phi}(r, h)$ e $\mathrm{E}_{\Phi}(r, h)$ dados na Proposição 1, considerando algumas distribuições da classe SMN.

- Distribuição Pearson tipo VII (e distribuição t de Student)

Temos que $U \sim \operatorname{Gamma}(\nu / 2, \delta / 2)$, $\operatorname{com} \nu>0$ e $\delta>0$. E para facilitar a notação, consideramos $\alpha_{1}=(\nu+2 r) / 2$ e $\alpha_{2}=\left(h^{2}+\delta\right) / 2$. Assim,

$$
\begin{aligned}
\mathrm{E}_{\phi}(r, h) & =\int_{0}^{\infty} \frac{\delta^{\frac{\nu}{2}} u^{\frac{\nu}{2}-1} u^{r}}{\sqrt{2 \pi} \Gamma\left(\frac{\nu}{2}\right) 2^{\frac{\nu}{2}}} \exp \left\{-\frac{u\left(h^{2}+\delta\right)}{2}\right\} d u \\
& =\frac{\Gamma\left(\frac{\nu+2 r}{2}\right) \delta^{\frac{\nu}{2}}\left(\frac{h^{2}+\delta}{2}\right)^{-\frac{\nu+2 r}{2}}}{\sqrt{2 \pi} \Gamma\left(\frac{\nu}{2}\right) 2^{\frac{\nu}{2}}} \times \int_{0}^{\infty} \frac{\alpha_{2}^{\alpha_{1}} u^{\prime\left\{\alpha_{1}-1\right\}}}{\Gamma\left(\alpha_{1}\right)} \exp \left\{-\alpha_{2} u^{\prime}\right\} d u^{\prime} \\
& =\frac{\Gamma\left(\frac{\nu+2 r}{2}\right)}{\sqrt{2 \pi} \Gamma\left(\frac{\nu}{2}\right)}\left(\frac{\delta}{2}\right)^{\nu / 2}\left(\frac{h^{2}+\delta}{2}\right)^{-\frac{\nu+2 r}{2}},
\end{aligned}
$$

em quea integral em (B.1) representa a fdp da variável aleatória $U^{\prime}$ com distribuição $\operatorname{Gamma}\left(\alpha_{1}, \alpha_{2}\right)$.

$$
\begin{aligned}
\mathrm{E}_{\Phi}(r, h) & =\int_{0}^{\infty} \frac{u^{\frac{2 r+\nu}{2}-1} \Phi\left(h u^{\frac{1}{2}}\right) \delta^{\frac{\nu}{2}}}{2^{\frac{\nu}{2}} \Gamma\left(\frac{\nu}{2}\right)} \exp \left\{-\frac{u \delta}{2}\right\} d u \\
& =\frac{\Gamma\left(\frac{\nu+2 r}{2}\right)}{\Gamma\left(\frac{\nu}{2}\right)}\left(\frac{\delta}{2}\right)^{-r} \times \int_{0}^{\infty}\left(\frac{\delta}{2}\right)^{\alpha_{1}} \frac{\Phi\left(h u^{\prime\left\{\frac{1}{2}\right\}}\right) u^{\prime\left\{\alpha_{1}-1\right\}}}{\Gamma\left(\alpha_{1}\right)} \exp \left\{-\frac{u^{\prime} \delta}{2}\right\} d u^{\prime} \\
& =\frac{\Gamma\left(\frac{\nu+2 r}{2}\right)}{\Gamma\left(\frac{\nu}{2}\right)}\left(\frac{\delta}{2}\right)^{-r} E_{U^{\prime}}\left[\Phi\left(h U^{\prime\left\{\frac{1}{2}\right\}}\right)\right] \\
& =\frac{\Gamma\left(\frac{\nu+2 r}{2}\right)}{\Gamma\left(\frac{\nu}{2}\right)}\left(\frac{\delta}{2}\right)^{-r} F_{P V I I}(h \mid \nu+2 r, \delta),
\end{aligned}
$$

em queem (B.2) obtemos a esperança relativa à $U^{\prime} \sim \operatorname{Gamma}\left(\alpha_{1}, \delta / 2\right)$ e $F_{P V I I}(\cdot)$ representa a fda da distribuição Pearson tipo VII. Assim, o resultado segue do Lema 2 .

Quando $\delta=\nu$, isto é, a distribuição t de Student, temos que $\mathrm{E}_{\phi}(r, h)$ e $\mathrm{E}_{\Phi}(r, h)$ são 
dados por

$$
\begin{aligned}
\mathrm{E}_{\phi}(r, h) & =\frac{\Gamma\left(\frac{\nu+2 r}{2}\right)}{\Gamma\left(\frac{\nu}{2}\right) \sqrt{2 \pi}}\left(\frac{\nu}{2}\right)^{\frac{\nu}{2}}\left(\frac{h^{2}+\nu}{2}\right)^{-\frac{(\nu+2 r)}{2}} ; \\
\mathrm{E}_{\Phi}(r, h) & =\frac{\Gamma\left(\frac{\nu+2 r}{2}\right)}{\Gamma\left(\frac{\nu}{2}\right)}\left(\frac{\nu}{2}\right)^{-r} F_{P V I I}(h \mid \nu+2 r, \nu) .
\end{aligned}
$$

\section{- Distribuição Slash}

Temos que $U \sim \operatorname{Beta}(\nu, 1)$, com $\nu$ sendo o parâmetro de forma positivo, e

$$
\begin{aligned}
E_{\phi}(r, h) & =\int_{0}^{1} u^{r} \frac{1}{\sqrt{2 \pi}} \exp \left\{-\frac{h^{2}}{2} u\right\} \nu u^{\nu-1} d u \\
& =\frac{\nu}{\sqrt{2 \pi}} \int_{0}^{1} u^{\nu+r-1} \exp \left\{-\frac{h^{2}}{2} u\right\} d u, \\
& =\frac{\nu}{\sqrt{2 \pi}}\left(\frac{h^{2}}{2}\right)^{-(\nu+r)} \gamma^{*}\left(\nu+r, \frac{h^{2}}{2}\right),
\end{aligned}
$$

assim utilizamos o Lema 3 para obter a equação (B.3).

$$
\begin{aligned}
E_{\Phi}(r, h) & =\int_{0}^{1} u^{r} \Phi\left(h u^{\frac{1}{2}}\right) \nu u^{\nu-1} d u \\
& =\frac{\nu}{\nu+r} \int_{0}^{1} \Phi\left(h u^{\prime\left\{\frac{1}{2}\right\}}\right) u^{\prime\{\nu+r-1\}}(\nu+r) d u^{\prime} \\
& =\frac{\nu}{\nu+r} F_{S L}(h \mid \nu+r),
\end{aligned}
$$

em quea integral em (B.4) é a esperança da função $\Phi\left(h U^{\prime\left\{\frac{1}{2}\right\}}\right)$ da variável aletória $U^{\prime} \sim \operatorname{Beta}(\nu+r, 1)$.

Usando o Lema 2, obtemos a equação (B.5), em que $F_{S L}(\cdot)$ é a fda da distribuição Slash.

\section{- Distribuição normal contaminada}

$$
\begin{aligned}
\mathrm{E}_{\phi}(r, h)= & u^{r} \phi\left(h u^{\frac{1}{2}}\right)\left[\nu \mathbb{I}_{\gamma}(u)+(1-\nu) \mathbb{I}_{1}(u)\right] \\
= & \nu \gamma^{r} \phi\left(h \gamma^{\frac{1}{2}}\right)+(1-\nu) \phi\left(h \gamma^{\frac{1}{2}}\right) ; \\
\mathrm{E}_{\Phi}(r, h)= & u^{r} \Phi\left(h u^{\frac{1}{2}}\right)\left[\nu \mathbb{I}_{\gamma}(u)+(1-\nu) \mathbb{I}_{1}(u)\right] \\
= & \nu \gamma^{r} \Phi\left(h \gamma^{\frac{1}{2}}\right)+(1-\nu) \Phi(h) \\
= & \gamma^{r}\left[\nu \Phi\left(h u^{\frac{1}{2}}\right)+(1-\nu) \Phi(h)\right] \\
& +(1-\nu)\left(1-\gamma^{r}\right) \Phi(h) \\
= & \gamma^{r} F_{C N}(h \mid \nu, \gamma)+(1-\nu)\left(1-\gamma^{r}\right) \Phi(h),
\end{aligned}
$$

em que $\mathbb{I}_{\gamma}(u)$ é a função indicadora de $\gamma$ e $F_{C N}(\cdot)$ é a fda da distribuição normal contaminada. 


\section{Apêndice $\mathrm{C}$}

\section{Detalhes do algoritmo EM}

Neste apêndice, mostramos como são obtidas as equações (2.10)-(2.12) do algoritmo EM.

Seja $\boldsymbol{\theta}=\left(\boldsymbol{\beta}^{\top}, \sigma^{2}, \boldsymbol{\nu}\right)$ o vetor com todos os parâmetros do modelo SMN-CR e considere a notação dada na Seção 2.4. Denotando a verossimilhança completa por $L\left(\cdot \mid \mathbf{y}_{\mathrm{obs}}, \mathbf{y}_{L}, \mathbf{u}\right)$ e a fdp por $f(\cdot)$, temos que

$$
\begin{aligned}
L\left(\boldsymbol{\theta} \mid \mathbf{y}_{\mathrm{obs}}, \mathbf{y}_{L}, \mathbf{u}\right) & =f\left(\mathbf{y}_{\mathrm{obs}}, \mathbf{y}_{L}, \mathbf{u}\right)=f\left(\mathbf{y}_{\mathrm{obs}}, \mathbf{y}_{L} \mid \mathbf{u}\right) h(\mathbf{u}) \\
& =f(\mathbf{y} \mid \mathbf{u}) h(\mathbf{u})=\prod_{i=1}^{n} f\left(y_{i} \mid u_{i}\right) h\left(u_{i} \mid \boldsymbol{\nu}\right) .
\end{aligned}
$$

Sem considerar constantes que não dependem dos parâmetros, a log verossimilhança completa é dada por

$$
\begin{aligned}
\ell_{c}\left(\boldsymbol{\theta} \mid \mathbf{y}_{\text {obs }}, \mathbf{y}_{L}, \mathbf{u}\right)= & \log \left(L\left(\boldsymbol{\theta} \mid \mathbf{y}_{\text {obs }}, \mathbf{y}_{L}, \mathbf{u}\right)\right) \\
& =\frac{n}{2} \log \left(\sigma^{2}\right)+\frac{1}{2} \sum_{i=1}^{n} \log \left(u_{i}\right)-\frac{1}{2 \sigma^{2}} \sum_{i=1}^{n} u_{i}\left(y_{i}-\mathbf{x}_{i}^{\top} \boldsymbol{\beta}\right)^{2}+\sum_{i=1}^{n} \log \left(h\left(u_{i} \mid \boldsymbol{\nu}\right)\right) .
\end{aligned}
$$

A função $Q$ no passo E do algoritmo é dado por

$$
Q\left(\boldsymbol{\theta} \mid \boldsymbol{\theta}^{(k)}\right)=\mathrm{E}_{\boldsymbol{\theta}^{(k)}}\left[\ell_{c}\left(\boldsymbol{\theta} \mid \mathbf{Y}_{\mathrm{obs}}, \mathbf{Y}_{L}, \mathbf{U}\right) \mid \mathbf{y}_{\mathrm{obs}}\right]
$$

assim, temos que

$$
\begin{aligned}
Q\left(\boldsymbol{\theta} \mid \boldsymbol{\theta}^{(k)}\right) & =-\frac{n}{2} \log \left(\sigma^{2}\right)-\frac{1}{2 \sigma^{2}} \sum_{i=1}^{n}\left\{\mathrm{E}_{\boldsymbol{\theta}^{(k)}}\left[U_{i} Y_{i}^{2} \mid y_{\mathrm{obs}_{i}}\right]\right. \\
& \left.-2 \mathrm{E}_{\boldsymbol{\theta}^{(k)}}\left[U_{i} Y_{i} \mid y_{\mathrm{obs}_{i}}\right] \mathbf{x}_{i}^{\top} \boldsymbol{\beta}+\mathrm{E}_{\boldsymbol{\theta}^{(k)}}\left[U_{i} \mid y_{\mathrm{obs}_{i}}\right]\left(\mathbf{x}_{i}^{\top} \boldsymbol{\beta}\right)^{2}\right\} \\
& +\frac{1}{2} \sum_{i=1}^{n} \mathrm{E}_{\boldsymbol{\theta}^{(k)}}\left[\log \left(U_{i}\right) \mid y_{\mathrm{obs}_{i}}\right]+\sum_{i=1}^{n} \mathrm{E}_{\boldsymbol{\theta}^{(k)}}\left[\log \left(h\left(U_{i} \mid \boldsymbol{\nu}\right)\right) \mid y_{\mathrm{obs}_{i}}\right] .
\end{aligned}
$$

As esperanças $\mathcal{E}_{s i}\left(\boldsymbol{\theta}^{(k)}\right)=\mathrm{E}_{\boldsymbol{\theta}^{(k)}}\left[U_{i} Y_{i}^{s} \mid y_{\mathrm{obs}_{i}}\right], \quad s=0,1,2$, usadas no passo E do algoritmo, são calculadas considerando dois possíveis casos, quando a $i$-ésima observação é (i) não censurada ou (ii) censurada. No primeiro caso, obtemos essas esperanças utilizando os resultados obtidos por Osorio et al. (2007) e no último caso, utilizamos os resultados obtidos 
na Proposição 1.

Assim, temos que

$$
\begin{aligned}
Q\left(\boldsymbol{\theta} \mid \boldsymbol{\theta}^{(k)}\right) & =-\frac{n}{2} \log \left(\sigma^{2}\right)-\frac{1}{2 \sigma^{2}} \sum_{i=1}^{n}\left[\mathcal{E}_{2 i}\left(\boldsymbol{\theta}^{(k)}\right)-2 \mathcal{E}_{1 i}\left(\boldsymbol{\theta}^{(k)}\right) \mathbf{x}_{i}^{\top} \boldsymbol{\beta}+\mathcal{E}_{0 i}\left(\boldsymbol{\theta}^{(k)}\right)\left(\mathbf{x}_{i}^{\top} \boldsymbol{\beta}\right)^{2}\right] \\
& +\frac{1}{2} \sum_{i=1}^{n} \mathrm{E}_{\boldsymbol{\theta}^{(k)}}\left[\log \left(U_{i}\right) \mid y_{\mathrm{obs}_{i}}\right]+\sum_{i=1}^{n} \mathrm{E}_{\boldsymbol{\theta}^{(k)}}\left[\log \left(h\left(U_{i} \mid \boldsymbol{\nu}\right)\right) \mid y_{\mathrm{obs}_{i}}\right] .
\end{aligned}
$$

No passo CM, obtemos as derivadas de $Q\left(\boldsymbol{\theta} \mid \boldsymbol{\theta}^{(k)}\right) \operatorname{sob} \boldsymbol{\beta}$ e $\sigma^{2}$, isto é,

$$
\begin{aligned}
& \frac{\partial Q\left(\boldsymbol{\theta} \mid \boldsymbol{\theta}^{(k)}\right)}{\partial \boldsymbol{\beta}}=\frac{1}{\sigma^{2}} \sum_{i=1}^{n}\left[\mathbf{x}_{i} \mathcal{E}_{1 i}\left(\boldsymbol{\theta}^{(k)}\right)-\mathcal{E}_{0 i}\left(\boldsymbol{\theta}^{(k)}\right) \mathbf{x}_{i} \mathbf{x}_{i}^{\top} \boldsymbol{\beta}\right] \\
& \frac{\partial Q\left(\boldsymbol{\theta} \mid \boldsymbol{\theta}^{(k)}\right)}{\partial \sigma^{2}}=-\frac{n}{2 \sigma^{2}}+\frac{1}{2 \sigma^{4}} \sum_{i=1}^{n}\left[\mathcal{E}_{2 i}\left(\boldsymbol{\theta}^{(k)}\right)-2 \mathcal{E}_{1 i}\left(\boldsymbol{\theta}^{(k)}\right) \mathbf{x}_{i}^{\top} \boldsymbol{\beta}+\mathcal{E}_{0 i}\left(\boldsymbol{\theta}^{(k)}\right)\left(\mathbf{x}_{i}^{\top} \boldsymbol{\beta}\right)^{2}\right] .
\end{aligned}
$$

A solução de $\frac{\partial Q\left(\boldsymbol{\theta} \mid \boldsymbol{\theta}^{(k)}\right)}{\partial \boldsymbol{\beta}}=0$ é

$$
\boldsymbol{\beta}^{(k+1)}=\left(\sum_{i=1}^{n} \mathcal{E}_{0 i}\left(\boldsymbol{\theta}^{(k)}\right) \mathbf{x}_{i} \mathbf{x}_{i}^{\top}\right)^{-1} \sum_{i=1}^{n} \mathbf{x}_{i} \mathcal{E}_{1 i}\left(\boldsymbol{\theta}^{(k)}\right) .
$$

A solução de $\frac{\partial Q\left(\boldsymbol{\theta} \mid \boldsymbol{\theta}^{(k)}\right)}{\partial \sigma^{2}}=0$ é

$$
\sigma^{2(k+1)}=\frac{1}{n} \sum_{i=1}^{n}\left[\mathcal{E}_{2 i}\left(\boldsymbol{\theta}^{(k)}\right)-2 \mathcal{E}_{1 i}\left(\boldsymbol{\theta}^{(k)}\right) \mathbf{x}_{i}^{\top} \boldsymbol{\beta}^{(k+1)}+\mathcal{E}_{0 i}\left(\boldsymbol{\theta}^{(k)}\right)\left(\mathbf{x}_{i}^{\top} \boldsymbol{\beta}^{(k+1)}\right)^{2}\right] .
$$

Para o passo CML, estimamos $\boldsymbol{\nu}$ maximizando a função de log verossimilhança marginal, com o intuito de contornar o cálculo de $\mathrm{E}_{\boldsymbol{\theta}^{(k)}}\left[\log \left(U_{i}\right) \mid y_{\mathrm{obs}_{i}}\right]$ e $\mathrm{E}_{\boldsymbol{\theta}^{(k)}}\left[\log \left(h\left(U_{i} \mid \boldsymbol{\nu}\right)\right) \mid y_{\mathrm{obs}_{i}}\right]$, isto é,

$$
\boldsymbol{\nu}^{(k+1)}=\operatorname{argmax}_{\boldsymbol{\nu}}\left\{\sum_{i=1}^{m} \log \left[F_{S M N}\left(\frac{\kappa_{i}-\mathbf{x}_{i}^{\top} \boldsymbol{\beta}^{(k+1)}}{\sigma^{(k+1)}}\right)\right] \sum_{i=m+1}^{n} \log \left[f_{S M N}\left(y_{i} \mid \mathbf{x}_{i}^{\top} \boldsymbol{\beta}^{(k+1)}, \sigma^{2(k+1)}, \boldsymbol{\nu}\right)\right]\right\} .
$$




\section{Apêndice D}

\section{Propriedades assintóticas}

As Figuras D.1 e D.2 mostram o Viés e o EQM médio das estimativas de MV de $\beta_{1}$, $\beta_{2}$ e $\sigma^{2}$ para os níveis de censura de $p=25 \%$ e $p=45 \%$, respectivamente.

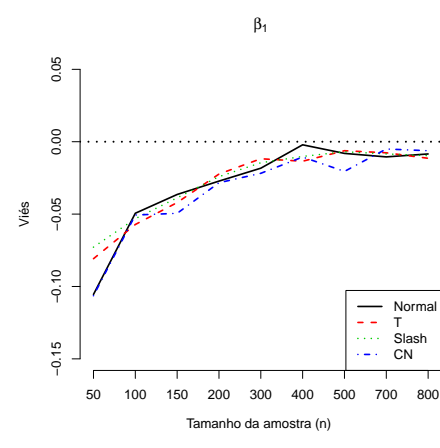

$\beta_{1}$

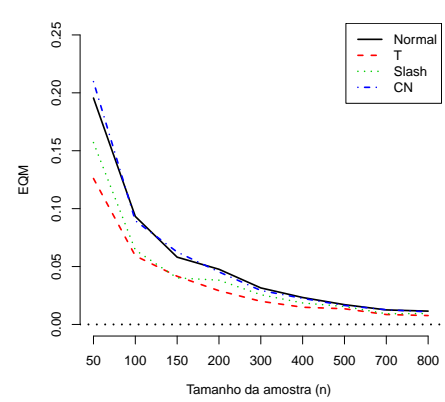

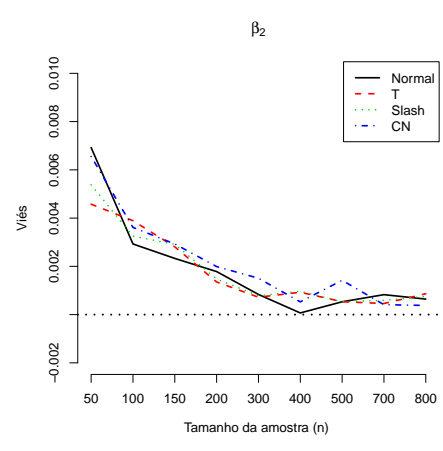

$\beta_{2}$

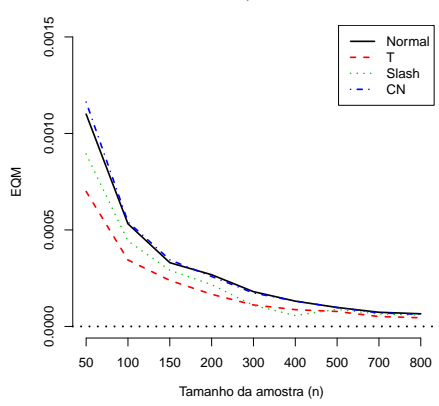

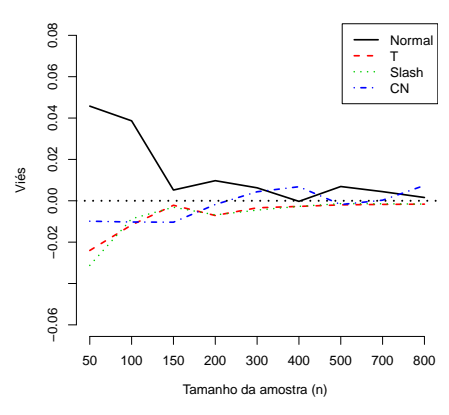

$\sigma^{2}$

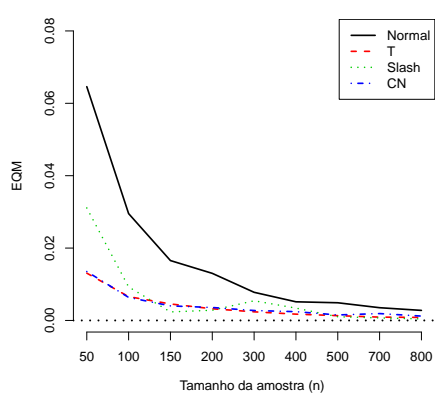

Figura D.1: Viés médio (primeira linha) e EQM (segunda linha) dos parâmetros estimados nos modelos SMN-CR, para $p=25 \%$. 


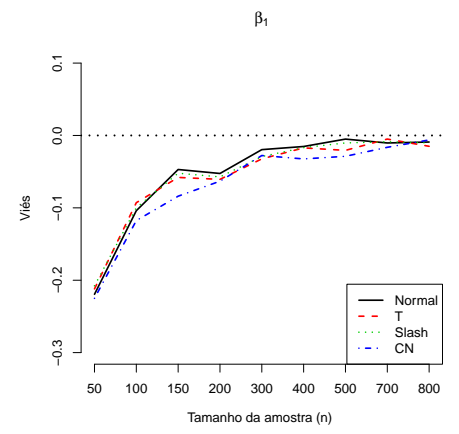

$\beta_{1}$

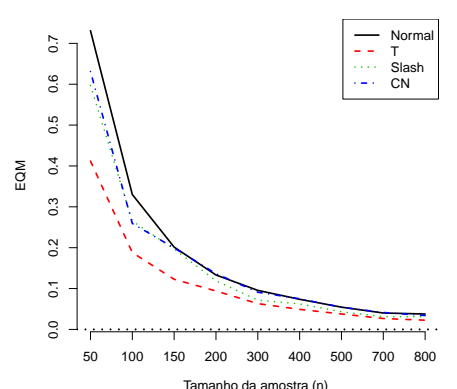

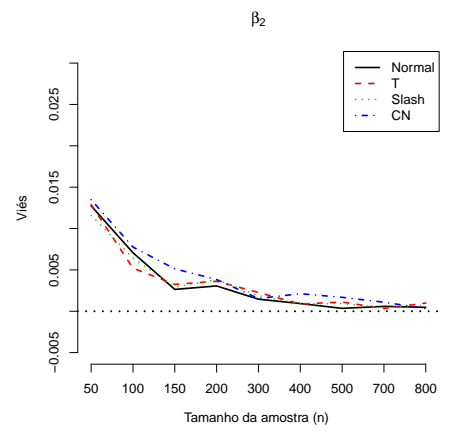

$\beta_{2}$

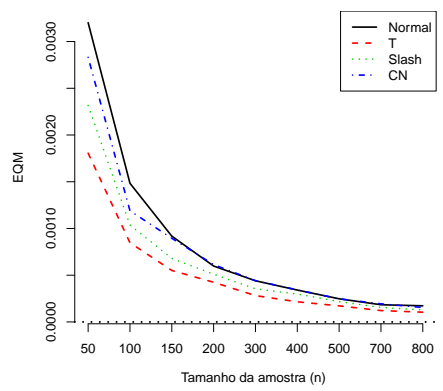

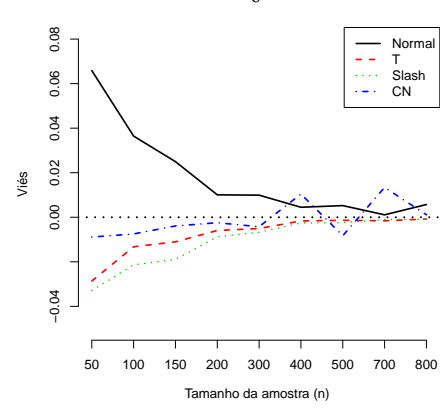

$\sigma^{2}$

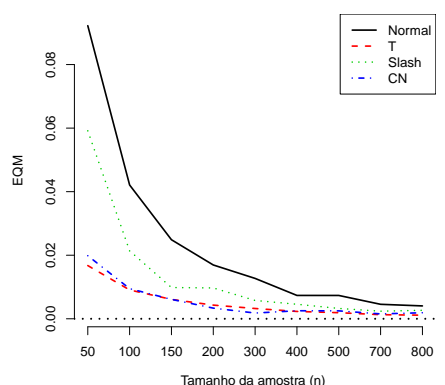

Figura D.2: Viés médio (primeira linha) e EQM (segunda linha) dos parâmetros estimados nos modelos SMN-CR, para $p=45 \%$. 


\section{Apêndice E}

\section{Algoritmo EM no modelo t-MLC}

Nesta seção, mostramos como são obtidas as equações (5.7)-(5.9) do algoritmo EM para o modelo t-MLC, definido na Seção 5.3.2. Seja $\mathbf{y}=\left(\mathbf{y}_{1}^{\top}, \ldots, \mathbf{y}_{n}^{\top}\right)^{\top}, \mathbf{u}=\left(u_{1}, \ldots, u_{n}\right)^{\top}$, $\mathbf{V}=\operatorname{vec}\left(\mathbf{V}_{1}, \ldots, \mathbf{V}_{n}\right)$, e $\mathbf{C}=\operatorname{vec}\left(\mathbf{C}_{1}, \ldots, \mathbf{C}_{n}\right)$, em que observamos $\left(\mathbf{V}_{i}, \mathbf{C}_{i}\right)$ para o $i$ ésimo caso. Considerando u e y como hipotéticos "dados perdidos", temos que os dados completos são $\mathbf{y}_{c}=\left(\mathbf{C}^{\top}, \mathbf{V}^{\top}, \mathbf{y}^{\top}, \mathbf{u}^{\top}\right)^{\top}$. Denotando a função de verossimilhança como $L\left(\cdot \mid \mathbf{C}^{\top}, \mathbf{V}^{\top}, \mathbf{y}^{\top}, \mathbf{u}^{\top}\right)$ e a função de densidade de probabilidade (fdp) por $f(\cdot)$, temos que para $\boldsymbol{\theta}=\left(\boldsymbol{\beta}^{\top}, \sigma^{2}, \boldsymbol{\phi}\right)^{\top}$,

$$
L\left(\boldsymbol{\theta} \mid \mathbf{C}^{\top}, \mathbf{V}^{\top}, \mathbf{y}^{\top}, \mathbf{u}^{\top}\right)=f(\mathbf{y} \mid \mathbf{V}, \mathbf{C}, \mathbf{u}) h(\mathbf{u})=f(\mathbf{y} \mid \mathbf{u}) h(\mathbf{u})=\prod_{i=1}^{n} f\left(\mathbf{y}_{i} \mid u_{i}\right) h\left(u_{i} \mid \boldsymbol{\nu}\right) .
$$

Assim, a função de log-verossimilhança completa é dada por

$$
\begin{aligned}
\ell_{c}\left(\boldsymbol{\theta} \mid \mathbf{y}_{c}\right) & =\log \left(L\left[\boldsymbol{\theta} \mid \mathbf{y}_{c}\right]\right)=\log \left(\prod_{i=1}^{n} f\left(\mathbf{y}_{i} \mid u_{i}\right) h\left(u_{i} \mid \nu\right)\right) \\
& =\sum_{i=1}^{n} \log \left((2 \pi)^{-p / 2} u_{i}^{1 / 2}\left|\boldsymbol{\Sigma}_{i}\right|^{-1 / 2} \exp \left(\frac{u_{i}}{2}\left(\mathbf{y}_{i}-\mathbf{X}_{i} \boldsymbol{\beta}\right)^{\top} \Sigma_{i}^{-1}\left(\mathbf{y}_{i}-\mathbf{X}_{i} \boldsymbol{\beta}\right)\right)\right) \\
& +\sum_{i=1}^{n} \log \left(h\left(u_{i} \mid \boldsymbol{\nu}\right)\right) \\
& =-\frac{1}{2} \sum_{i=1}^{n}\left[n_{i} \log \left(\sigma^{2}\right)+\log \left(\left|\mathbf{E}_{i}\right|\right)+\frac{u_{i}}{\sigma^{2}}\left(\mathbf{y}_{i}-\mathbf{X}_{i} \boldsymbol{\beta}\right)^{\top} \mathbf{E}_{i}^{-1}\left(\mathbf{y}_{i}-\mathbf{X}_{i} \boldsymbol{\beta}\right)\right] \\
& +\sum_{i=1}^{n} \log \left(h\left(u_{i} \mid \nu\right)\right)+c,
\end{aligned}
$$

em que $c$ é uma constante que não depende do vetor de parâmetros $\boldsymbol{\theta}$ e $h\left(u_{i} \mid \nu\right)$ é a densidade Gamma $(\nu / 2, \nu / 2)$.

Assim, nosso algoritmo tipo EM para o modelo t-MLC pode ser resumido da seguinte forma,

\section{Passo E:}

Dado os valores atualizados $\boldsymbol{\theta}=\widehat{\boldsymbol{\theta}}^{(k)}$, o passo E calcula a esperança condicional da função 
de log-verossimilhança completa

$$
Q\left(\boldsymbol{\theta} \mid \widehat{\boldsymbol{\theta}}^{(k)}\right)=\sum_{i=1}^{n} Q_{i}\left(\boldsymbol{\theta} \mid \widehat{\boldsymbol{\theta}}^{(k)}\right)=\sum_{i=1}^{n}\left\{-\frac{n_{i}}{2} \log \left(\sigma^{2}\right)-\frac{1}{2} \log \left(\left|\mathbf{E}_{i}\right|\right)-\frac{1}{2 \sigma^{2}} A_{i}^{(k)}(\boldsymbol{\beta}, \boldsymbol{\phi})\right\},
$$

com

$$
A_{i}^{(k)}(\boldsymbol{\beta}, \boldsymbol{\phi})=\left[\operatorname{tr}\left({\widehat{u \mathbf{y}_{i}^{2}}}^{(k)} \mathbf{E}_{i}^{-1}\right)-2 \boldsymbol{\beta} \mathbf{X}_{i}^{\top} \mathbf{E}_{i}^{-1} \widehat{u \mathbf{y}}_{i}^{(k)}+\widehat{u}_{i}^{(k)} \boldsymbol{\beta} \mathbf{X}_{i}^{\top} \mathbf{E}_{i}^{-1} \mathbf{X}_{i} \boldsymbol{\beta}\right]
$$

Note que neste caso não consideramos o cálculo de $E\left[\log \left(h\left(u_{i} \mid \nu\right)\right) \mid \mathbf{V}, \mathbf{C}, \widehat{\boldsymbol{\theta}}^{(k)}\right]$, pois $\nu$ é fixado.

\section{Passo CM:}

Atualizamos $\widehat{\boldsymbol{\theta}}^{(k)}$ pela maximização de $Q\left(\boldsymbol{\theta} \mid \widehat{\boldsymbol{\theta}}^{(k)}\right)$ em relação a $\boldsymbol{\theta}=\left(\boldsymbol{\beta}^{\top}, \sigma^{2}, \boldsymbol{\phi}\right)^{\top}$, em que

$$
\begin{aligned}
& \frac{\partial Q\left(\boldsymbol{\theta} \mid \boldsymbol{\theta}^{(k)}\right)}{\partial \boldsymbol{\beta}}=\frac{1}{\sigma^{2}} \sum_{i=1}^{n}\left[\mathbf{X}_{i}^{\top}\left(\widehat{\mathbf{E}}_{i}^{(k)}\right)^{-1} \widehat{u \mathbf{y}}_{i}^{(k)}-\left(\widehat{u}_{i}^{(k)} \mathbf{X}_{i}^{\top}\left(\widehat{\mathbf{E}}_{i}^{(k)}\right)^{-1} \mathbf{X}_{i}\right) \boldsymbol{\beta}\right] \\
& \frac{\partial Q\left(\boldsymbol{\theta} \mid \boldsymbol{\theta}^{(k)}\right)}{\partial \sigma^{2}}=-\frac{N}{2 \sigma^{2}}+\frac{1}{2 \sigma^{4}} \sum_{i=1}^{n} A_{i}^{(k)}\left(\widehat{\boldsymbol{\beta}}^{(k+1)}, \widehat{\boldsymbol{\phi}}^{(k)}\right) .
\end{aligned}
$$

Desta forma, a solução de $\frac{\partial Q\left(\boldsymbol{\theta} \mid \boldsymbol{\theta}^{(k)}\right)}{\partial \boldsymbol{\beta}}=0$ e $\frac{\partial Q\left(\boldsymbol{\theta} \mid \boldsymbol{\theta}^{(k)}\right)}{\partial \sigma^{2}}=0$ é dada por

$$
\begin{aligned}
\widehat{\boldsymbol{\beta}}^{(k+1)} & =\left(\sum_{i=1}^{n} \widehat{u}_{i}^{(k)} \mathbf{X}_{i}^{\top}\left(\widehat{\mathbf{E}}_{i}^{(k)}\right)^{-1} \mathbf{X}_{i}\right)^{-1} \sum_{i=1}^{n} \mathbf{X}_{i}^{\top}\left(\widehat{\mathbf{E}}_{i}^{(k)}\right)^{-1} \widehat{u \mathbf{y}}_{i}^{(k)}, \\
{\widehat{\sigma^{2}}}^{(k+1)} & =\frac{1}{N} \sum_{i=1}^{n} A_{i}^{(k)}\left(\widehat{\boldsymbol{\beta}}^{(k+1)}, \widehat{\boldsymbol{\phi}}^{(k)}\right),
\end{aligned}
$$

em que $N=\sum_{i=1}^{n} n_{i}$. Com o intuito de evitar o calculo de $\frac{\partial \mathbf{E}_{i}}{\partial \phi}$, estimamos $\phi$ pela maximização da função de verossimilhança marginal. Esta estratégia foi utilizada por Wang \& Fan (2010) e Wang (2013), entre outros autores. Assim,

$$
\widehat{\boldsymbol{\phi}}^{(k+1)}=\operatorname{argmax}_{\boldsymbol{\phi}}\left\{-\frac{1}{2} \sum_{i=1}^{n}\left[\log \left(\left|\mathbf{E}_{i}\right|\right)+A_{i}^{(k)}\left(\widehat{\boldsymbol{\beta}}^{(k+1)}, \boldsymbol{\phi}\right)\right]\right\},
$$

Este processo é iterado até que algum critério de convergência seja satisfeito. Por exemplo, quando a distância envolvendo duas avaliações sucessivas da log-verossimilhança $\mid \ell\left(\widehat{\boldsymbol{\theta}}^{(k+1)}\right) / \ell\left(\widehat{\boldsymbol{\theta}}^{(k)}\right)-$ 1|, for suficientemente pequena. 


\section{Apêndice F}

\section{Prova das Proposições 3 e 4}

Neste Seção apresentaremos as provas das Proposições 3 e 4 que serão úteis na implementação do nosso algoritmo tipo EM para o modelo t-MLC.

\section{Prova da Proposição 3:}

Seja $d(\mathbf{y}, \boldsymbol{\theta})=(\mathbf{Y}-\boldsymbol{\mu})^{\top} \boldsymbol{\Sigma}^{-1}(\mathbf{Y}-\boldsymbol{\mu}) \operatorname{com} \boldsymbol{\theta}=(\boldsymbol{\mu}, \boldsymbol{\Sigma}, \nu)$ e $c_{p}(\nu, r)=\left(\frac{\nu+p}{\nu}\right)^{r}\left(\frac{\Gamma\left(\frac{\nu+p}{2}\right) \Gamma\left(\frac{\nu+2 r}{2}\right)}{\Gamma\left(\frac{\nu}{2}\right) \Gamma\left(\frac{\nu+p+2 r}{2}\right)}\right)$, então temos que:

$$
\begin{aligned}
& \left(\frac{\nu+p}{\nu+d(\mathbf{y}, \boldsymbol{\theta})}\right)^{r} t_{p}(\mathbf{y} \mid \boldsymbol{\mu}, \boldsymbol{\Sigma}, \nu)=\left(\frac{\nu+p}{\nu+d(\mathbf{y}, \boldsymbol{\theta})}\right)^{r} \frac{\Gamma\left(\frac{\nu+p}{2}\right)}{\Gamma\left(\frac{\nu}{2}\right) \pi^{\frac{p}{2}}} \nu^{-\frac{p}{2}}|\boldsymbol{\Sigma}|^{-1 / 2}\left(1+\frac{d(\mathbf{y}, \boldsymbol{\theta})}{\nu}\right)^{-\frac{\nu+p}{2}} \\
& =c_{p}(\nu, r) \frac{\Gamma\left(\frac{\nu+p+2 r}{2}\right)}{\Gamma\left(\frac{\nu+2 r}{2}\right)} \frac{(\nu+2 r)^{-\frac{p}{2}}}{\pi^{\frac{p}{2}}}\left|\frac{\nu}{\nu+2 r} \boldsymbol{\Sigma}\right|^{-\frac{1}{2}}\left(1+\frac{d(\mathbf{y}, \boldsymbol{\theta})}{\nu}\right)^{-\frac{\nu+p+2 r}{2}} \\
& =c_{p}(\nu, r) \frac{\Gamma\left(\frac{\nu+p+2 r}{2}\right)}{\Gamma\left(\frac{\nu+2 r}{2}\right)} \frac{(\nu+2 r)^{-\frac{p}{2}}}{\pi^{\frac{p}{2}}}\left|\boldsymbol{\Sigma}^{*}\right|^{-\frac{1}{2}}\left(1+\frac{d^{*}(\mathbf{y}, \boldsymbol{\theta})}{\nu+2 r}\right)^{-\frac{\nu+p+2 r}{2}} \\
& =c_{p}(\nu, r) t_{p}\left(\mathbf{y} \mid \boldsymbol{\mu}, \boldsymbol{\Sigma}^{*}, \nu+2 r\right) \text {, } \\
& \text { em que } \quad \boldsymbol{\Sigma}^{*}=\left(\frac{\nu}{\nu+2 r}\right) \boldsymbol{\Sigma} \quad \text { e } \quad d^{*}(\mathbf{y}, \boldsymbol{\theta})=(\mathbf{Y}-\boldsymbol{\mu})^{\top} \boldsymbol{\Sigma}^{*-1}(\mathbf{Y}-\boldsymbol{\mu}), \quad \text { assim } \\
& \left(\frac{\nu+p}{\nu+d(\mathbf{y}, \boldsymbol{\theta})}\right)^{r} t_{p}(\mathbf{y} \mid \boldsymbol{\mu}, \boldsymbol{\Sigma}, \nu) \frac{T_{p}(\mathbf{a} \mid \boldsymbol{\mu}, \boldsymbol{\Sigma}, \nu)}{T_{p}(\mathbf{a} \mid \boldsymbol{\mu}, \boldsymbol{\Sigma}, \nu)} \mathbb{I}_{\mathbb{A}}(\mathbf{y})=c_{p}(\nu, r) t_{p}\left(\mathbf{y} \mid \boldsymbol{\mu}, \boldsymbol{\Sigma}^{*}, \nu+2 r\right) \frac{T_{p}\left(\mathbf{a} \mid \boldsymbol{\mu}, \boldsymbol{\Sigma}^{*}, \nu+2 r\right)}{T_{p}\left(\mathbf{a} \mid \boldsymbol{\mu}, \boldsymbol{\Sigma}^{*}, \nu+2 r\right)} \mathbb{I}_{\mathbb{A}}(\mathbf{y}) \\
& \left(\frac{\nu+p}{\nu+d(\mathbf{y}, \boldsymbol{\theta})}\right)^{r} T t_{p}(\mathbf{y} \mid \boldsymbol{\mu}, \boldsymbol{\Sigma}, \nu ; \mathbb{A})=c_{p}(\nu, r) \frac{T_{p}\left(\mathbf{a} \mid \boldsymbol{\mu}, \boldsymbol{\Sigma}^{*}, \nu+2 r\right)}{T_{p}(\mathbf{a} \mid \boldsymbol{\mu}, \boldsymbol{\Sigma}, \nu)} T t_{p}\left(\mathbf{y} \mid \boldsymbol{\mu}, \boldsymbol{\Sigma}^{*}, \nu+2 r\right) .
\end{aligned}
$$


Prova da Proposição 4: Seja $\mathbf{Y} \sim t_{p}(\boldsymbol{\mu}, \boldsymbol{\Sigma}, \nu)$ particionada como $\mathbf{Y}^{\top}=\left(\mathbf{Y}_{1}^{\top}, \mathbf{Y}_{2}^{\top}\right)^{\top}$ $\operatorname{com} \operatorname{dim}\left(\mathbf{Y}_{1}\right)=p_{1}, \quad \operatorname{dim}\left(\mathbf{Y}_{2}\right)=p_{2}$ e $p_{1}+p_{2}=p$, então, pela Proposição 2, temos que $\mathbf{Y}_{2} \mid \mathbf{Y}_{1}=\mathbf{y}_{1} \sim t_{p_{2}}\left(\boldsymbol{\mu}_{2.1}, \widetilde{\boldsymbol{\Sigma}}_{22.1}, \nu+p_{1}\right)$, em que $\widetilde{\boldsymbol{\Sigma}}_{22.1}=\left(\frac{\nu+d\left(\mathbf{y}_{1}, \boldsymbol{\theta}_{1}\right)}{\nu+p_{1}}\right) \boldsymbol{\Sigma}_{22.1}$, com $d\left(\mathbf{y}_{1}, \boldsymbol{\theta}_{1}\right)=\left(\mathbf{y}_{1}-\boldsymbol{\mu}_{1}\right)^{\top} \boldsymbol{\Sigma}_{11}^{-1}\left(\mathbf{y}_{1}-\boldsymbol{\mu}_{1}\right), \boldsymbol{\Sigma}_{22.1}=\boldsymbol{\Sigma}_{22}-\boldsymbol{\Sigma}_{21} \boldsymbol{\Sigma}_{11}^{-1} \boldsymbol{\Sigma}_{12}$ e $\boldsymbol{\mu}_{2.1}=\boldsymbol{\mu}_{2}+\boldsymbol{\Sigma}_{21} \boldsymbol{\Sigma}_{11}^{-1}\left(\mathbf{y}_{1}-\right.$ $\left.\boldsymbol{\mu}_{1}\right)$.

Assim, considerando $\widetilde{d}_{2.1}\left(\mathbf{y}_{2}, \boldsymbol{\theta}_{2}\right)=\left(\mathbf{y}_{2}-\boldsymbol{\mu}_{2.1}\right)^{\top} \widetilde{\boldsymbol{\Sigma}}_{22.1}^{-1}\left(\mathbf{y}_{2}-\boldsymbol{\mu}_{2.1}\right)$ e $d_{2.1}\left(\mathbf{y}_{2}, \boldsymbol{\theta}_{2}\right)=\left(\mathbf{y}_{2}-\right.$ $\left.\boldsymbol{\mu}_{2.1}\right)^{\top} \boldsymbol{\Sigma}_{22.1}^{-1}\left(\mathbf{y}_{2}-\boldsymbol{\mu}_{2.1}\right)$ temos que:

$$
\begin{aligned}
& \left(\frac{\nu+p}{\nu+d(\mathbf{y}, \boldsymbol{\theta})}\right)^{r} \frac{\left(\nu+d\left(\mathbf{y}_{1}, \boldsymbol{\theta}_{1}\right)\right)^{r}}{d_{p}\left(p_{1}, \nu, r\right)} t_{p_{2}}\left(\mathbf{y}_{2} \mid \boldsymbol{\mu}_{2.1}, \widetilde{\boldsymbol{\Sigma}}_{22.1}, \nu+p_{1}\right) \\
& =\left(\frac{\nu+p}{\nu+d(\mathbf{y}, \boldsymbol{\theta})}\right)^{r} \frac{\left(\nu+d\left(\mathbf{y}_{1}, \boldsymbol{\theta}_{1}\right)\right)^{r}}{d_{p}\left(p_{1}, \nu, r\right)} \frac{\Gamma\left(\frac{\nu+p}{2}\right)}{\Gamma\left(\frac{\nu+p_{1}}{2}\right)} \frac{\left(\nu+p_{1}\right)^{-\frac{p_{2}}{2}}}{\pi^{\frac{p_{2}}{2}}}\left|\widetilde{\boldsymbol{\Sigma}}_{22.1}\right|^{-\frac{1}{2}}\left(1+\frac{\widetilde{d}_{2.1}\left(\mathbf{y}_{2}, \boldsymbol{\theta}_{2}\right)}{\nu+p_{1}}\right)^{-\frac{\nu+p}{2}} \\
& =\left(\frac{\nu+d\left(\mathbf{y}_{1}, \boldsymbol{\theta}_{1}\right)}{\nu+d(\mathbf{y}, \boldsymbol{\theta})}\right)^{r} \frac{\Gamma\left(\frac{p+\nu+2 r}{2}\right)}{\Gamma\left(\frac{\nu+p_{1}+2 r}{2}\right)} \frac{\left(\nu+d\left(\mathbf{y}_{1}, \boldsymbol{\theta}_{1}\right)\right)^{-\frac{p_{2}}{2}}}{\pi^{\frac{p_{2}}{2}}}\left|\boldsymbol{\Sigma}_{22.1}\right|^{-\frac{1}{2}}\left(1+\frac{\widetilde{d}_{2.1}\left(\mathbf{y}_{2}, \boldsymbol{\theta}_{2}\right)}{\nu+p_{1}}\right)^{-\frac{\nu+p}{2}}
\end{aligned}
$$

sabendo que $d_{2.1}\left(\mathbf{y}_{2}, \boldsymbol{\theta}_{2}\right)=d(\mathbf{y}, \boldsymbol{\theta})-d\left(\mathbf{y}_{1}, \boldsymbol{\theta}_{1}\right)$,

$$
\begin{aligned}
& =\frac{\Gamma\left(\frac{\nu+p+2 r}{2}\right)}{\Gamma\left(\frac{\nu+p_{1}+2 r}{2}\right)} \frac{\left(\nu+p_{1}+2 r\right)^{-\frac{p_{2}}{2}}}{\pi^{\frac{p_{2}}{2}}}\left|\widetilde{\boldsymbol{\Sigma}}_{22.1}^{*}\right|^{-\frac{1}{2}}\left(\frac{\nu+d(\mathbf{y}, \boldsymbol{\theta})}{\nu+d\left(\mathbf{y}_{1}, \boldsymbol{\theta}_{1}\right)}\right)^{-\frac{\nu+p+2 r}{2}} \\
& =\frac{\Gamma\left(\frac{\nu+p+2 r}{2}\right)}{\Gamma\left(\frac{\nu+p_{1}+2 r}{2}\right)} \frac{\left(\nu+p_{1}+2 r\right)^{-\frac{p_{2}}{2}}}{\pi^{\frac{p_{2}}{2}}}\left|\widetilde{\boldsymbol{\Sigma}}_{22.1}^{*}\right|^{-\frac{1}{2}}\left(1+\frac{\widetilde{d}_{2.1}^{*}\left(\mathbf{y}_{2}, \boldsymbol{\theta}_{2}\right)}{\nu+p_{1}+2 r}\right)^{-\frac{\nu+p+2 r}{2}} \\
& =t_{p_{2}}\left(\mathbf{y}_{2} \mid \boldsymbol{\mu}_{2.1}, \widetilde{\boldsymbol{\Sigma}}_{22.1}^{*}, \nu+p_{1}+2 r\right) .
\end{aligned}
$$

Assim, considerando truncamento temos que

$$
\begin{aligned}
\left(\frac{\nu+p}{\nu+d(\mathbf{y}, \boldsymbol{\theta})}\right)^{r} T t_{p_{2}}\left(\mathbf{y}_{2} \mid \boldsymbol{\mu}_{2.1}, \widetilde{\boldsymbol{\Sigma}}_{22.1}, \nu+p_{1} ; \mathbb{A}^{y_{2}}\right) & =\frac{d_{p}\left(\nu, p_{1}, r\right)}{\left(\nu+d\left(\mathbf{y}_{1}, \boldsymbol{\theta}_{1}\right)\right)^{r}} \frac{T_{p_{2}}\left(\mathbf{a}^{y_{2}} \mid \boldsymbol{\mu}_{2.1}, \widetilde{\boldsymbol{\Sigma}}_{22.1}^{*}, \nu+p_{1}+2 r\right)}{T_{p_{2}}\left(\mathbf{a}^{y_{2}} \mid \boldsymbol{\mu}_{2.1}, \widetilde{\boldsymbol{\Sigma}}_{22.1}, \nu+p_{1}\right)} \\
& \times T t_{p_{2}}\left(\mathbf{y}_{2} \mid \boldsymbol{\mu}_{2.1}, \widetilde{\boldsymbol{\Sigma}}_{22.1}^{*}, \nu+p_{1}+2 r ; \mathbb{A}^{y_{2}}\right) .
\end{aligned}
$$

Portanto, $E\left[\left(\frac{\nu+p}{\nu+d(\mathbf{y}, \boldsymbol{\theta})}\right)^{r} \mathbf{Y}_{2}^{(k)} \mid \mathbf{Y}_{1}\right]=\frac{d_{p}\left(\nu, p_{1}, r\right)}{\left(\nu+d\left(\mathbf{y}_{1}, \boldsymbol{\theta}_{1}\right)\right)^{r}} \frac{T_{p_{2}}\left(\mathbf{a}^{y_{2}} \mid \boldsymbol{\mu}_{2.1}, \widetilde{\boldsymbol{\Sigma}}_{22.1}^{*}, \nu+p_{1}+2 r\right)}{T_{p_{2}}\left(\mathbf{a}^{y_{2}} \mid \boldsymbol{\mu}_{2.1}, \widetilde{\boldsymbol{\Sigma}}_{22.1}, \nu+p_{1}\right)} E\left[\mathbf{Y}_{\mathbf{2}}^{*}(k)\right]$, em que $\mathbf{Y}_{2}^{*} \sim T t_{p_{2}}\left(\boldsymbol{\mu}_{2.1}, \widetilde{\boldsymbol{\Sigma}}_{22.1}^{*}, \nu+p_{1}+2 r ; \mathbb{A}^{y^{2}}\right)$. 


\section{Referências Bibliográficas}

Akaike, H. (1974). A new look at the statistical model identification. Automatic Control, IEEE Transactions on, 19, 716-723.

Ando, T. (2010). Bayesian Model Selection and Statistical Modeling. Chapman \& Hal/CRC.

Andrews, D. F. \& Mallows, C. L. (1974). Scale mixtures of normal distributions. Journal of the Royal Statistical Society. Series B (Methodological), 36, 99-102.

Arellano-Valle, R., Castro, L., González-Farías, G. \& Muñoz-Gajardo, K. (2012). Student-t censored regression model: properties and inference. Statistical Methods $\&$ Applications, 21, 453-473.

Arellano-Valle, R. B. (1994). Distribuições Elípticas: Propriedades, Inferência e Aplicações a Modelos de Regressão. Tese de doutorado, Instituto de Matemática e Estatística, Universidade de São Paulo. in portuguese.

Arellano-Valle, R. B. \& Bolfarine, H. (1995). On some characterizations of the t-distribution. Statistics 83 Probability Letters, 25, 79-85.

Arnold, B. C. \& Press, S. J. (1989). Bayesian estimation and prediction for pareto data. Journal of the American Statistical Association, 84, 1079-1084.

Bai, Z. D., Krishnaiah, P. R. \& Zhao, L. C. (1989). On rates of convergence of efficient detection criteria in signal processing with white noise. IEEE Trans. Info. Theory, 35, 380-388.

Bandyopadhyay, D., Lachos, V., Castro, L. \& Dey, D. (2012). Skew-normal/independent linear mixed models for censored responses with applications to HIV viral loads. Biometrical Journal, 54, 405-425.

Barros, M., Galea, M., González, M. \& Leiva, V. (2010). Influence diagnostics in the Tobit censored response model. Statistical Methods $\&$ Applications, 19, 716-723.

Basso, R. M., Lachos, V. H., Cabral, C. R. B. \& Ghosh, P. (2010). Robust mixture modeling based on scale mixtures of skew-normal distributions. Computational Statistics \& Data Analysis, 54, 2926 - 2941. 
Branco, M. D. \& Dey, D. K. (2001). A general class of multivariate skew-elliptical distributions. Journal of Multivariate Analysis, 79, 99-113.

Branco, M. D., Genton, M. C. \& Liseo, B. (2012). Objective Bayesian analysis of skew-t distributions. Scandinavian Journal of Statistics, doi:10.1111/j.14679469.2011.00779.x.

Breen, R. (1996). Regression Models: Censored, Sample Selected, or Truncated Data. Sage Publications.

Brooks, S. P. (2002). Discussion on the paper by Spiegelhalter, Best, Carlin, and van der Linde (2002). 64, 616-618.

Browne, W. \& Goldstein, H. (2010). MCMC sampling for a multilevel model with nonindependent residuals within and between cluster units. Journal of Educational and Behavioral Statistics, 35, 453-473.

Cabral, C. R. B., Lachos, V. H. \& Madruga, M. R. (2012). Bayesian analysis of skew-normal independent linear mixed models with heterogeneity in the random-effects population. Journal of Statistical Planning and Inference, 142, 181-200.

Cancho, V. C., Lachos, V. H. \& Ortega, E. M. M. (2009). A nonlinear regression model with skew-normal errors. Statistical Papers, 51, 547-551.

Carlin, B. P. \& Louis, T. A. (2001). Bayes and Empirical Bayes Methods for Data Analysis. Chapman \& Hall/CRC, Boca Raton, second edition.

Casella, G. \& George, E. I. (1992). Explaining the gibbs sampler. The American Statistician, 46, 167-174.

Castro, L. M., Lachos, V. H. \& Arellano-Valle, R. B. (2014). Partially linear censored regression models using heavy-tailed distributions: a Bayesian approach. Statistical Methodology, 18, 14-31.

Chen, M., Huang, L., Ibrahim, J. \& Kim, S. (2008). Bayesian variable selection and computation for generalized linear models with conjugate priors. Bayesian Analysis, 3, 585-614.

Chib, S. (1992). Bayes inference in the Tobit censored regression model. Journal of Econometrics, 51, 79-99.

Colosimo, E. \& Giolo, S. (2006). Análise de sobrevivência aplicada. ABE - Projeto Fisher. ISBN 9788521203841.

Csiszar, I. (1967). Information-type measures of difference of probability distributions and indirect observations. Studia Scientiarum Mathematicarum Humgarica, pages 299-318.

Cysneiros, F. J. A. \& Vanegas, L. H. (2008). Residuals and their statistical properties in symmetrical nonlinear models. Statistics \& Probability Letters, 78, 3269-3273. 
Dempster, A., Laird, N. \& Rubin, D. (1977). Maximum likelihood from incomplete data via the EM algorithm. Journal of the Royal Statistical Society, Series B, 39, 1-38.

Dey, D. K., Chen, M. H. \& Chang, H. (1997). Bayesian approach for the nonlinear random effects models. Biometrics, 53, 1239-1252.

Dueker, M. J. (2006). Kalman filtering with truncated normal state variables for bayesian estimation of macroeconomic models. Econ Lett, 93, 58-62.

Fagundes, R. A., de Souza, R. M. \& Cysneiros, F. J. A. (2013). Robust regression with application to symbolic interval data. Engineering Applications of Artificial Intelligence, 26, 564-573.

Fang, K. T. \& Zhang, Y. T. (1990). Generalized Multivariate Analysis. Springer.

Fonseca, T. C. O., Ferreira, M. A. R. \& Migon, H. S. (2008). Objective Bayesian analysis for the Student-t regression model. Biometrika, 95, 325-333.

Gamerman, D. \& Lopes, H. F. (2006). Markov Chain Monte Carlo: Stochastic Simulation for Bayesian Inference. Chapman \& Hall/CRC, second edition.

Garay, A. M. \& Lachos, V. H. (2013). BayesCR: Bayesian analysis of censored linear regression models with scale mixtures of normal (SMN) distributions. $\mathrm{R}$ package version 1.0 .

Garay, A. M., Lachos, V. H. \& Massuia, M. B. (2013). SMNCensReg: Fitting univariate censored regression model under the scale mixture of normal distributions. $\mathrm{R}$ package version 2.3 .

Gelfand, A. E. (1995). Gibbs sampling. Journal of the American Statistical Association, 452, 1300-1304.

Gelfand, A. E., Hills, S. E., Racine-Poon, A. \& Smith, A. F. M. (1990). Illustration of bayesian inference in normal data models using gibbs sampling. Journal of the American Statistical Association, 85, 972-985.

Gelman, A. \& Rubin., D. B. (1992). Inference from iterative simulation using multiple sequences. Statistical science, 7, 457-472.

Gelman, A., Carlin, J. B., Stern, H. S. \& Rubin, D. B. (2004). Bayesian data analysis. Chapman \& Hall/CRC.

Geman, S. \& Geman, D. (1984). Stochastic relaxation, Gibbs distributions, and the Bayesian restoration of images. IEEE Transactions on Pattern Analysis and Machine Intelligence, pages $721-741$.

Genç, A. (2013). Moments of truncated normal/independent distributions. Statistical Papers, 54, 741-764. 
Genz, A., Bretz, F., Hothorn, T., Miwa, T., Mi, X., Leisch, F. \& Scheipl, F. (2008). mvtnorm: Multivariate Normal and t Distribution. $R$ package version 0.9-2, URL http://CRAN. R-project. org/package= mvtnorm.

Geweke, J. (1993). Bayesian treatment of the independent Student-t linear model. Journal of Applied Econometrics, 8, S19-S40.

Goldstein, H., Healy, M. \& Rasbash, J. (1994). Multilevel time series models with applications to repeated measures data. Statistics in Medicine, 13, 1643-1655.

Greene, W. (2012). Econometric Analysis. Prentice Hall.

Hastings, W. K. (1970). Monte carlo sampling methods using markov chains and their applications. Biometrika, 57, 97-109.

Heuchenne, C. \& Keilegom, I. V. (2007). Nonlinear Regression with Censored Data. Technometrics, 49, 34-44.

Ibacache-Pulgar, G. \& Paula, G. (2011). Local influence for Student-t partially linear models. Computational Statistics \& Data Analysis, 55, 1462-1478.

Jawitz, J. W. (2004). Moments of truncated continuous univariate distributions. Advances in Water Resources, 27, 269-281.

Kalbfleisch, J. \& Lawless, J. (1992). Some useful statistical methods for truncated data. Journal of Quality and Technology, 24, 145-152.

Kim, H. J. (2008). Moments of truncated Student- distribution. Journal of the Korean Statistical Society, 37, 81-87.

Labra, F. V., Garay, A. M., Lachos, V. H. \& Ortega, E. M. M. (2012). Estimation and diagnostics for heteroscedastic nonlinear regression models based on scale mixtures of skew-normal distributions. Journal of Statistical Planning and Inference, 142, 21492165.

Lachos, V., Bandyopadhyay, D. \& Dey, D. (2011). Linear and nonlinear mixed-effects models for censored HIV viral loads using normal/independent distributions. Biometrics, 67, 1594-1604.

Lachos, V., Castro, L. \& Dey, D. (2013). Bayesian inference in nonlinear mixed-effects models using normal independent distributions. Computational Statistical \& Data Analysis, 64, 237-252.

Laird, N. M. \& H.Ware, J. (1982). Random effects models for longitudinal data. Biometrics, 38, 963-974.

Lange, K. L., Little, R. \& Taylor, J. (1989). Robust statistical modeling using t distribution. Journal of the American Statistical Association, 84, 881-896. 
Lee, G. \& Scott, C. (2012). EM algorithms for multivariate gaussian mixture models with truncated and censored data. Computational Statistics \& Data Analysis, 56, 2816 - 2829.

Liang, H. \& Zou, G. (2008). Improved AIC selection strategy for survival analysis. Computational Statistics \& Data Analysis, 52, 2538-2548.

Lin, J., Xie, F. \& Wei, B. (2009). Statistical diagnostics for skew-t-normal nonlinear models. Communications in Statistics-Simulation and Computation, 38, 2096-2110.

Lin, T. \& Lee, J. (2007). Bayesian analysis of hierarchical linear mixed modeling using the multivariate t distribution. Journal of Statistical Planning and Inference, 137, 484-495.

Lin, T., Ho, H., Chen, H. \& Wang, W. (2011). Some results on the truncated multivariate $\mathrm{t}$ distribution. Journal of Statistical Planning and Inference, 142, 25-40.

Liu, C. \& Rubin, D. B. (1994). The ECME algorithm: A simple extension of EM and ECM with faster monotone convergence. Biometrika, 80, 267-278.

Liu, J. S. (1994). The collapsed Gibbs sampler in Bayesian computations with applications to a gene regulation problem. Journal of the American Statistical Association, 89, 958966.

Louis, T. A. (1982). Finding the observed information matrix when using the EM algorithm. Journal of the Royal Statistical Society, Series B, pages 226-233.

Lucas, A. (1997). Robustness of the Student $\mathrm{t}$ based M-estimator. Communications in Statistics, Theory and Methods, 26, 1165-1182.

Martinez-Florez, G., Bolfarine, H. \& Gómez, H. W. (2013). The Alpha-power tobit model. Communications in Statistics - Theory and Methods, 42, 633-643.

Massuia, M. B., Cabral, C. R. B., Matos, L. A. \& Lachos, V. H. (2012). Influence diagnostics for Student-t censored linear regression models. Technical Report 09-23, IMECC, Universidade Estadual de Campinas.

Matos, L. A., Prates, M. O., Chen, M. H. \& Lachos, V. H. (2013). Likelihood-based inference for mixed-effects models with censored response using the multivariate-t distribution. Statistica Sinica, 23, 1323-1342.

Meng, X. L. \& Rubin, B. D. (1993). Maximum likelihood estimation via the ECM algorithm: A general framework. Biometrika, 80, 267-278.

Metropolis, N., Rosenbluth, A. W., Rosenbluth, M. N., Teller, A. H. \& Teller, E. (1953). Equation of State Calculations by Fast Computing Machines. The Journal of Chemical Physics, 21, 1087-1092.

Meza, C., Osorio, F. \& la Cruz, R. D. (2012). Estimation in nonlinear mixed-effects models using heavy-tailed distributions. Statistics and Computing, 22, 121-139. 
Mroz, T. A. (1987). The sensitivity of an empirical model of married women's hours of work to economic and statistical assumptions. Econometrica, 55, 765-799.

Muñoz, A., Carey, V., Schouten, J. P., Segal, M. \& Rosner, B. (1992). A parametric family of correlation structures for the analysis of longitudinal data. Biometrics, pages 733-742.

Ndembi, N., Goodall, R., Dunn, D., McCormick, A., Burke, A., Lyagoba, F., Munderi, P., Katundu, P., Kityo, C., Robertson, V., Yirrell, D., Walker, A., Gibb, D., Gilks, C., Kaleebu, P. \& Pillay, D. (2010). Viral rebound and emergence of drug resistance in the absence of viral load testing: A randomized comparison between Zidovudine-Lamivudine plus Nevirapine and Zidovudine-Lamivudine plus Abacavir. Journal of Infectious Diseases, 201, $106-113$.

Nelson, F. D. (1977). Censored regression models with unobserved, stochastic censoring thresholds. Journal of Econometrics, 6, 309-327.

Nelson, W. (1990). Hazard plotting of left truncated life data. Journal of Quality and Technology, 22, 230-238.

Ortega, E. M. M., Bolfarine, H. \& Paula, G. A. (2003). Influence diagnostics in generalized log-gamma regression models. Computational Statistics \& Data Analysis, 42, 165-186.

Osorio, F., Paula, G. A. \& Galea, M. (2007). Assessment of local influence in elliptical linear models with longitudinal structure. Computational Statistics \& Data Analysis, 51, $4354-4368$.

Park, J. W., Genton, M. G. \& Ghosh, S. K. (2007). Censored time series analysis with autoregressive moving average models. Canadian Journal of Statistics, 35, 151-168.

Peng, F. \& Dey, D. K. (1995). Bayesian analysis of outlier problems using divergence measures. The Canadian Journal of Statistics, 23, 199-213.

Pinheiro, J. C. \& Bates, D. M. (2000). Mixed-Effects Models in S and S-PLUS. Springer, New York, NY.

Pinheiro, J. C., Liu, C. H. \& Wu, Y. N. (2001). Efficient algorithms for robust estimation in linear mixed-effects models using a multivariate t-distribution. Journal of Computational and Graphical Statistics, 10, 249-276.

Qiu, W. \& Wu, L. (2010). HIV Viral Dynamic Models With Censoring and Informative Dropouts. Statistics in Biopharmaceutical Research, 2, 220-228.

R Core Team (2013). R: A Language and Environment for Statistical Computing. R Foundation for Statistical Computing, Vienna, Austria.

Rao, C. R. (1973). Linear statistical inference and its applications, second Edition,. John Wiley \& Sons. 
Rao, C. R. (1987). Prediction of future observations in growth curve models. Statistical Science, 2, 434-447.

Robert, C. P. \& Casella, G. (2004). Monte Carlo Statistical Methods. Springer.

Rosa, G. J. M., Padovani, C. R. \& Gianola, D. (2003). Robust linear mixed models with normal/independent distributions and Bayesian MCMC implementation. Biometrical Journal, 45, 573-590.

Saitoh, A., Foca, M., Viani, R. M., Heffernan-Vacca, S., Vaida, F., Lujan-Zilbermann, J., Emmanuel, P. J., Deville, J. G. \& Spector, S. A. (2008). Clinical outcomes after an unstructured treatment interruption in children and adolescents with perinatally acquired HIV infection. Pediatrics, 121, 513-521.

Schwarz, G. (1978). Estimating the dimension of a model. Annals of Statistics, 6, 461-464.

Spiegelhalter, D. J., Best, N. G., Carlin, B. P. \& van der Linde, A. (2002). Bayesian measures of model complexity and fit. Journal of the Royal Statistical Society, Series B, 64, 583-639.

Stapleton, D. C. \& Young, J. D. (1984). Censored normal regression with measurement error on the dependent variable. Econometrica, pages 737-760.

Therneau, T. M., Grambsch, P. M. \& Fleming, R. T. (1990). Martingale-based residuals for survival models. Biometrika, pages 147-160.

Thompson, M. L. \& Nelson, K. P. (2003). Linear regression with Type I interval and left-censored response data. Environmental and Ecological Statistics, 10, 221-230.

Vaida, F. \& Liu, L. (2009). Fast Implementation for Normal Mixed Effects Models With Censored Response. Journal of Computational and Graphical Statistics, 18, 797-817.

Vaida, F. \& Liu, L. (2012). lmec: Linear Mixed-Effects Models with Censored Responses. $R$ Package Version 1.0 URL http://cran.r-project.org/web/packages/lmec.

Vaida, F., Fitzgerald, A. P. \& DeGruttola, V. (2007). Efficient hybrid EM for linear and nonlinear mixed effects models with censored response. Computational Statistics $\&$ Data Analysis, 51, 5718-5730.

Vanegas, L. H. \& Cysneiros, F. J. A. (2010). Assessment of diagnostic procedures in symmetrical nonlinear regression models. Computational Statistics 63 Data Analysis, 54, $1002-1016$.

Vanegas, L. H., Rondón, L. M. \& Cysneiros, F. J. A. (2012). Diagnostic procedures in Birnbaum-Saunders nonlinear regression models. Computational Statistics $\&$ Data Analysis, 56, 1662-1680. 
Vidal, I. \& Castro, L. M. (2010). Influential observations in the independent Student-t measurement error model with weak nondifferential error. Chilean Journal of Statistics, $1,17-34$.

Villegas, C., Paula, G., Cysneiros, F. \& Galea, M. (2012). Influence diagnostics in generalized symmetric linear models. Computational Statistics \& Data Analysis, 59, 161-170.

Wang, W. \& Fan, T. (2011). Estimation in multivariate t linear mixed models for multivariate longitudinal data. Statistica Sinica, 21, 1857-1880.

Wang, W.-L. (2013). Multivariate t linear mixed models for irregularly observed multiple repeated measures with missing outcomes. Biometrical Journal, 55, 554-571.

Wang, W.-L. \& Fan, T.-H. (2010). ECM-based maximum likelihood inference for multivariate linear mixed models with autoregressive errors. Computational Statistics \& Data Analysis, 54, 1328-1341.

Wei, C. G. \& Tanner, M. A. (1990). Posterior computations for censored regression data. Journal of the American Statistical Association, 85, 829-839.

Wu, L. (2010). Mixed Effects Models for Complex Data. Chapman \& Hall/CRC.

Wu, L., Liu, W. \& Hu, X. (2010). Joint Inference on HIV Viral Dynamics and Immune Suppression in Presence of Measurement Errors. Biometrics, pages 327-335. 